\title{
Avaliação de técnicas de diagnóstico para a análise de dados com medidas repetidas
}

\author{
Ricardo Salles Kurusu
}

\author{
DisSERTAÇÃO APRESENTADA \\ $\mathrm{AO}$ \\ Instituto DE MATEMÁtica E EstatísticA \\ DA \\ Universidade DE SÃO PAULO \\ PARA \\ OBTENÇÃO DO TÍTULO \\ $\mathrm{DE}$ \\ Mestre em CiênCiAs
}

Programa: Estatística

Orientador: Prof ${ }^{\mathrm{a}}$. Dr ${ }^{\mathrm{a}}$. Denise Aparecida Botter

Durante o desenvolvimento deste trabalho o autor recebeu auxílio financeiro da CAPES

São Paulo, Junho de 2013 



\section{Avaliação de técnicas de diagnóstico para a análise de dados com medidas repetidas}

Esta versão da dissertação contém as correções e alterações sugeridas pela Comissão Julgadora durante a defesa da versão original do trabalho, realizada em 26/04/2013. Uma cópia da versão original está disponível no Instituto de Matemática e Estatística da Universidade de São Paulo.

Comissão Julgadora:

- Prof ${ }^{a}$. Dr ${ }^{a}$. Denise Aparecida Botter (orientadora) - IME-USP

- Prof ${ }^{a}$. Dra . Viviana Giampaoli - IME-USP

- Prof. Dr. Juvêncio Santos Nobre - UFC 



\section{Agradecimentos}

Primeiramente gostaria de agradecer a todos os meus familiares. Em especial aos meus pais, que me apoiaram e estiveram sempre do meu lado. À minha irmã Renata por participar diretamente ao assistir à minha defesa. Ao meu irmão Rafael juntamente com a Mary, que me ajudaram bastante mesmo estando tão longe.

Às professoras Denise Aparecida Botter e Mônica Carneiro Sandoval, pela orientação, dedicação e paciência que tiveram comigo durante todo esse tempo. Foi ótimo ter tido a oportunidade de trabalhar novamente com as duas.

Aos professores da banca, Viviana Giampaoli e Juvêncio Santos Nobre, pelas críticas, elogios e sugestões que me ajudaram a finalizar o meu trabalho da melhor maneira possível. Ao colega Roberto Manghi que, da mesma forma que o Juvêncio, disponibilizou as rotinas do R utilizadas em seu trabalho.

Aos professores e funcionários do IME que participaram diretamente da minha formação desde os tempos da graduação. Assim como os amigos que fiz durante esse período na USP. Eu considero um privilégio poder ter convivido e aprendido com essas pessoas.

Aos colegas de trabalho que me acompanharam durante a parte final dessa jornada.

Por fim, agradeço à CAPES pelo apoio financeiro durante parte desse período. 


\section{Resumo}

\section{Avaliação de técnicas de diagnóstico para a análise de dados com medidas repetidas}

Dentre as possíveis propostas encontradas na literatura estatística para analisar dados oriundos de estudos com observações correlacionadas, estão os modelos condicionais e os modelos marginais. Diversas técnicas têm sido propostas para a análise de diagnóstico nesses modelos. O objetivo deste trabalho é apresentar algumas das técnicas de diagnóstico disponíveis para os dois tipos de modelos e avaliá-las por meio de estudos de simulação. As técnicas apresentadas também foram aplicadas em um conjunto de dados reais.

Palavras-chave: equações de estimação generalizadas, medidas repetidas, modelos condicionais, modelos lineares generalizados hierárquicos, modelos lineares generalizados mistos, modelos lineares mistos, modelos marginais, técnicas de diagnóstico. 


\section{Abstract}

Evaluation of diagnostic techniques for the analysis of data with repeated measures

Conditional and marginal models are among the possibilities in statistical literature to analyze data from studies with correlated observations. Several techniques have been proposed for diagnostic analysis in these models. The objective of this work is to present some of the diagnostic techniques available for both modeling approaches and to evaluate them by simulation studies. The presented techniques were also applied in a real dataset.

Keywords: conditional models, diagnostic techniques, generalized estimating equations, generalized linear mixed models, hierarchical generalized linear models, linear mixed models, marginal models, repeated measures. 


\section{Sumário}

Lista de Figuras $\quad$ ix

Lista de Tabelas $\quad$ xv

1 Introdução 1

2 Modelos Condicionais 5

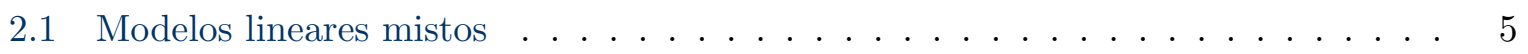

2.1.1 Estimação em modelos lineares mistos . . . . . . . . . . . . . . . 8

2.2 Modelos lineares generalizados com efeitos aleatórios . . . . . . . . . . . . . 9

2.2.1 Modelos lineares generalizados mistos . . . . . . . . . . . . . . . 9

2.2.2 Modelos lineares generalizados hierárquicos . . . . . . . . . . . . . . 10

2.3 Técnicas de diagnóstico f . . . . . . . . . . . . . . . . . . . . . . 13

2.3.1 Diagnóstico em modelos lineares mistos . . . . . . . . . . . . . . . 13

2.3.2 Diagnóstico em modelos lineares generalizados mistos e hierárquicos . . 20

3 Modelos Marginais $\quad 23$

3.1 Equações de estimação generalizadas . . . . . . . . . . . . . . . . . . . . . 23

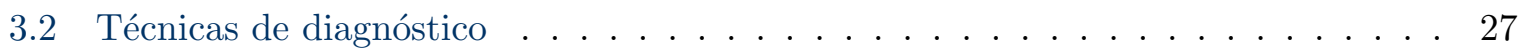

4 Simulações $\quad 29$

4.1 Modelos com intercepto aleatório . . . . . . . . . . . . . . . . . . . . 29

4.2 Modelos com inclinação aleatória . . . . . . . . . . . . . . . . . . . 61

4.3 Dados sem independência condicional . . . . . . . . . . . . . 76

4.4 Recursos computacionais . . . . . . . . . . . . . . . . . 85

$\begin{array}{llr}5 & \text { Aplicação } & 87\end{array}$

6 Conclusões $\quad 105$

$\begin{array}{ll}\text { A Rotinas computacionais } & 107\end{array}$

$\begin{array}{ll}\text { Referências Bibliográficas } & 121\end{array}$ 


\section{Lista de Figuras}

4.1 Resíduos marginais e condicionais padronizados versus os índices das unidades experimentais do modelo linear misto com intercepto aleatório - estudo 1. . . . . 31

4.2 Resíduos marginais e condicionais padronizados versus os valores ajustados do modelo linear misto com intercepto aleatório - estudo 1. . . . . . . . . . . . 32

4.3 Gráfico dos resíduos para a estrutura de covariância versus os índices das unidades experimentais do modelo linear misto com intercepto aleatório - estudo 1. . . . . 32

4.4 Gráficos Q-Q dos resíduos marginais e condicionais padronizados do modelo linear misto com intercepto aleatório - estudo 1. . . . . . . . . . . . 33

4.5 Gráfico Q-Q dos resíduos minimamente confundidos do modelo linear misto com intercepto aleatório - estudo $1 . \ldots \ldots \ldots \ldots$

4.6 Gráfico Q-Q dos resíduos $t_{i j}^{*}$ do modelo linear misto com intercepto aleatório estudo $\ldots \ldots \ldots \ldots \ldots \ldots \ldots \ldots$

4.7 Gráfico Q-Q dos resíduos quantílicos do modelo linear misto com intercepto aleatório - estudo 1. . . . . . . . . . . . . . . . . . . 34

4.8 Gráficos das distâncias de Mahalanobis do modelo linear misto com intercepto aleatório - estudo 1. . . . . . . . . . . . . . . . 35

4.9 Gráficos das medidas de alavanca com base em $\boldsymbol{H}^{m}$ versus os índices das unidades experimentais do modelo linear misto com intercepto aleatório - estudo 1. . . . . 35

4.10 Gráficos das medidas de alavanca com base em $\boldsymbol{H}^{c}$ versus os índices das unidades experimentais do modelo linear misto com intercepto aleatório - estudo 1. . . . . 36

4.11 Gráfico das distâncias de Cook versus os índices das unidades experimentais do modelo linear misto com intercepto aleatório - estudo 1. . . . . . . . . . . 36

4.12 Gráficos dos resíduos padronizados do modelo marginal normal com estrutura de correlação uniforme - estudo 1. . . . . . . . . . . . . . . . . . . . 37

4.13 Gráficos das medidas de alavanca com base em $\boldsymbol{H}^{g}$ versus os índices das unidades experimentais do modelo marginal normal com estrutura de correlação uniforme - estudo 1. . . . . . . . . . . . . . . . . . . . . . . 37

4.14 Gráfico das distâncias de Cook versus os índices das unidades experimentais do modelo marginal normal com estrutura de correlação uniforme - estudo 1. . . . . 38

4.15 Resíduos marginais e condicionais padronizados versus os índices das unidades experimentais do modelo linear misto com intercepto aleatório - estudo 2. . . . . 40

4.16 Resíduos marginais e condicionais padronizados versus os valores ajustados do

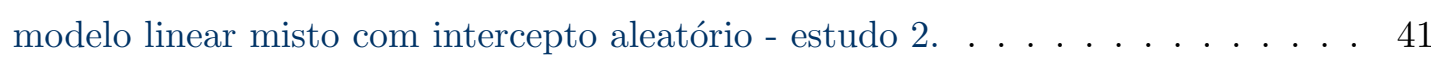


4.17 Gráfico dos resíduos para a estrutura de covariância versus os índices das unidades experimentais do modelo linear misto com intercepto aleatório - estudo 2. . . . . 41

4.18 Gráficos Q-Q dos resíduos marginais e condicionais padronizados do modelo linear misto com intercepto aleatório - estudo 2. . . . . . . . . . . . . . . . . . . . 42

4.19 Gráfico Q-Q dos resíduos minimamente confundidos do modelo linear misto com intercepto aleatório - estudo 2. . . . . . . . . . . . . . . . . . . . . . . . . . 42

4.20 Gráfico Q-Q dos resíduos $t_{i j}^{*}$ do modelo linear misto com intercepto aleatório estudo 2. . . . . . . . . . . . . . . . . . . . . . . . 43

4.21 Gráfico Q-Q dos resíduos quantílicos do modelo linear misto com intercepto aleatório - estudo 2. . . . . . . . . . . . . . . . . . . . . . . . .

4.22 Gráficos das distâncias de Mahalanobis do modelo linear misto com intercepto aleatório - estudo 2. . . . . . . . . . . . . . . . . . . . . . . . .

4.23 Gráficos das medidas de alavanca com base em $\boldsymbol{H}^{m}$ versus os índices das unidades experimentais do modelo linear misto com intercepto aleatório - estudo 2 . . . . .

4.24 Gráficos das medidas de alavanca com base em $\boldsymbol{H}^{c}$ versus os índices das unidades experimentais do modelo linear misto com intercepto aleatório - estudo 2. . . . . 45

4.25 Gráfico das distâncias de Cook versus os índices das unidades experimentais do modelo linear misto com intercepto aleatório - estudo 2. . . . . . . . . . .

4.26 Gráficos dos resíduos padronizados do modelo marginal normal com estrutura de correlação uniforme - estudo 2. . . . . . . . . . . . . . . . . . . . 46

4.27 Gráficos das medidas de alavanca com base em $\boldsymbol{H}^{g}$ versus os índices das unidades experimentais do modelo marginal normal com estrutura de correlação uniforme - estudo 2. . . . . . . . . . . . . . . . . . . . . . . . . . 46 46

4.28 Gráfico das distâncias de Cook versus os índices das unidades experimentais do modelo marginal normal com estrutura de correlação uniforme - estudo 2. . . . 47

4.29 Resíduos marginais e condicionais padronizados versus os índices das unidades experimentais do modelo linear misto com intercepto aleatório - estudo 3. . . . . 50

4.30 Resíduos marginais e condicionais padronizados versus os valores ajustados do modelo linear misto com intercepto aleatório - estudo 3. . . . . . . . . . . . . . . 51

4.31 Gráfico dos resíduos para a estrutura de covariância versus os índices das unidades experimentais do modelo linear misto com intercepto aleatório - estudo 3. . . . . 51

4.32 Gráficos Q-Q dos resíduos marginais e condicionais padronizados do modelo linear misto com intercepto aleatório - estudo 3. . . . . . . . . . . . . . . . . . . . 52

4.33 Gráfico Q-Q dos resíduos minimamente confundidos do modelo linear misto com intercepto aleatório - estudo 3. . . . . . . . . . . . . . . . . 52

4.34 Gráfico Q-Q dos resíduos $t_{i j}^{*}$ do modelo linear misto com intercepto aleatório estudo 3. . . . . . . . . . . . . . . . . . . . . . . . 53

4.35 Gráfico Q-Q dos resíduos quantílicos do modelo linear misto com intercepto aleatório - estudo 3. . . . . . . . . . . . . . . . . . . . . 53

4.36 Gráficos das distâncias de Mahalanobis do modelo linear misto com intercepto aleatório - estudo 3. . . . . . . . . . . . . . . . . . . 54

4.37 Gráficos das medidas de alavanca com base em $\boldsymbol{H}^{m}$ versus os índices das unidades experimentais do modelo linear misto com intercepto aleatório - estudo 3. . . . . 54 
4.38 Gráficos das medidas de alavanca com base em $\boldsymbol{H}^{c}$ versus os índices das unidades experimentais do modelo linear misto com intercepto aleatório - estudo 3 . . . . . 55

4.39 Gráfico das distâncias de Cook versus os índices das unidades experimentais do modelo linear misto com intercepto aleatório - estudo 3. . . . . . . . . . . . 55

4.40 Gráficos dos resíduos padronizados do modelo marginal normal com estrutura de correlação uniforme - estudo 3. . . . . . . . . . . . . . . . . . . . . 56

4.41 Gráficos das medidas de alavanca com base em $\boldsymbol{H}^{g}$ versus os índices das unidades experimentais do modelo marginal normal com estrutura de correlação uniforme - estudo 3. . . . . . . . . . . . . . . . . . . . . . 56

4.42 Gráfico das distâncias de Cook versus os índices das unidades experimentais do modelo marginal normal com estrutura de correlação uniforme - estudo 3. . . . 57

4.43 Gráficos dos resíduos padronizados do modelo marginal gama com estrutura de correlação uniforme - estudo 3. . . . . . . . . . . . . . . . . . . 57

4.44 Gráficos das medidas de alavanca com base em $\boldsymbol{H}^{g}$ versus os índices das unidades experimentais do modelo marginal gama com estrutura de correlação uniforme estudo 3. . . . . . . . . . . . . . . . . . . . . . .

4.45 Gráfico das distâncias de Cook versus os índices das unidades experimentais do modelo marginal gama com estrutura de correlação uniforme - estudo 3. . . . . .

4.46 Gráficos dos componentes do desvio padronizados do modelo linear generalizado hierárquico gama com intercepto aleatório - estudo 3. . . . . . . . . . . . . . .

4.47 Gráfico Q-Q dos resíduos $t_{i j}^{*}$ do modelo linear generalizado hierárquico gama com intercepto aleatório - estudo 3.

4.48 Gráfico Q-Q dos resíduos quantílicos do modelo linear generalizado hierárquico

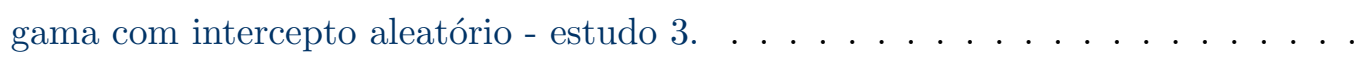

4.49 Gráficos das medidas de alavanca com base em $\boldsymbol{H}^{a}$ versus os índices das unidades experimentais do modelo linear generalizado hierárquico gama com intercepto aleatório - estudo 3. . . . . . . . . . . . . . . . . . . . .

4.50 Resíduos marginais e condicionais padronizados versus os índices das unidades experimentais do modelo com intercepto aleatório para a simulação com inclinação aleatória. ...........................

4.51 Resíduos marginais e condicionais padronizados versus os valores ajustados do modelo com intercepto aleatório para a simulação com inclinação aleatória. . . .

4.52 Gráfico Q-Q dos resíduos para a estrutura de covariância do modelo com intercepto aleatório para a simulação com inclinação aleatória. . . . . . . . . . . . . .

4.53 Gráficos Q-Q dos resíduos marginais e condicionais padronizados do modelo com intercepto aleatório para a simulação com inclinação aleatória. . . . . . . . . . .

4.54 Gráfico Q-Q dos resíduos minimamente confundidos do modelo com intercepto aleatório para a simulação com inclinação aleatória. . . . . . . . . . . . . . . . .

4.55 Gráfico Q-Q dos resíduos $t_{i j}^{*}$ do modelo com intercepto aleatório para a simulação com inclinação aleatória. . . . . . . . . . . . . . . . . . . . . 66

4.56 Gráfico dos resíduos quantílicos do modelo com intercepto aleatório para a simulação com inclinação aleatória. . . . . . . . . . . . . . . . . . . 
4.57 Gráficos das distâncias de Mahalanobis do modelo com intercepto aleatório para a simulação com inclinação aleatória. . . . . . . . . . . . . . . . . . . . 67

4.58 Gráficos das medidas de alavanca com base em $\boldsymbol{H}^{m}$ versus os índices das unidades experimentais do modelo com intercepto aleatório para a simulação com inclinação aleatória. . . . . . . . . . . . . . . . . .

4.59 Gráficos das medidas de alavanca com base em $\boldsymbol{H}^{c}$ versus os índices das unidades experimentais do modelo com intercepto aleatório para a simulação com inclinação aleatória.

4.60 Gráfico das distâncias de Cook versus os índices das unidades experimentais do modelo com intercepto aleatório para a simulação com inclinação aleatória. . . . .

4.61 Resíduos marginais e condicionais padronizados versus os índices das unidades experimentais do modelo com intercepto e inclinação aleatórios. . . . . . . . . . .

4.62 Resíduos marginais e condicionais padronizados versus os valores ajustados do modelo com intercepto e inclinação aleatórios. . . . . . . . . . . . . . .

4.63 Gráfico Q-Q dos resíduos para a estrutura de covariância do modelo com intercepto e inclinação aleatórios. . . . . . . . . . . . . . . . . .

4.64 Gráficos Q-Q dos resíduos marginais e condicionais padronizados do modelo com intercepto e inclinação aleatórios. . . . . . . . . . . . . . . . . . . . . 70

4.65 Gráfico Q-Q dos resíduos minimamente confundidos do modelo com intercepto e inclinação aleatórios. . . . . . . . . . . . . . . . . . . . . 71

4.66 Gráfico dos resíduos $t_{i j}^{*}$ do modelo com intercepto e inclinação aleatórios. . . . . 71

4.67 Gráfico dos resíduos quantílicos do modelo com intercepto e inclinação aleatórios. 72

4.68 Gráficos das distâncias de Mahalanobis do modelo com intercepto e inclinação

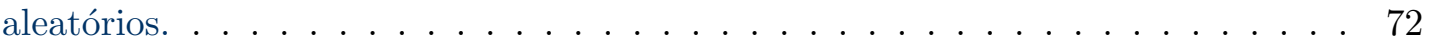

4.69 Gráficos das medidas de alavanca com base em $\boldsymbol{H}^{m}$ versus os índices das unidades experimentais do modelo com intercepto e inclinação aleatórios. . . . . . . . . . . 73

4.70 Gráficos das medidas de alavanca com base em $\boldsymbol{H}^{c}$ versus os índices das unidades experimentais do modelo com intercepto e inclinação aleatórios. . . . . . . . . . . 73

4.71 Gráfico das distâncias de Cook versus os índices das unidades experimentais do modelo com intercepto e inclinação aleatórios. . . . . . . . . . . . . . . . . . . . 74

4.72 Gráficos dos resíduos do modelo marginal normal com matriz de correlações não estruturada para a simulação com intercepto e inclinação aleatórios. . . . . . . . .

4.73 Gráficos das medidas de alavanca com base em $\boldsymbol{H}^{g}$ versus os índices das unidades experimentais do modelo marginal normal com matriz de correlações não estruturada para a simulação com intercepto e inclinação aleatórios. . . . . . . . . .

4.74 Gráfico das distâncias de Cook versus os índices das unidades experimentais do modelo marginal normal com matriz de correlações não estruturada para a simulação com intercepto e inclinação aleatórios. . . . . . . . . . . . . . . . .

4.75 Resíduos marginais e condicionais padronizados versus os índices das unidades experimentais do modelo linear misto com intercepto aleatório na simulação com estrutura AR-1. . . . . . . . . . . . . . . . . .

4.76 Resíduos marginais e condicionais padronizados versus os valores ajustados do modelo linear misto com intercepto aleatório na simulação com estrutura AR-1. . 
4.77 Gráfico para a estrutura de covariância do modelo linear misto com intercepto aleatório na simulação com estrutura AR-1. . . . . . . . . . . . . . . . . . . . . . 80

4.78 Gráficos Q-Q dos resíduos marginais e condicionais padronizados do modelo linear misto com intercepto aleatório na simulação com estrutura AR-1. . . . . . . . . . 81

4.79 Gráfico Q-Q dos resíduos minimamente confundidos do modelo linear misto com intercepto aleatório na simulação com estrutura AR-1. . . . . . . . . . . . . . . . 81

4.80 Gráfico Q-Q de $t_{i j}^{*}$ do modelo linear misto com intercepto aleatório na simulação com estrutura AR-1. . . . . . . . . . . . . . . . . . . . . 82

4.81 Gráfico Q-Q dos resíduos quantílicos do modelo linear misto com intercepto aleatório na simulação com estrutura AR-1. . . . . . . . . . . . . . . . . . . . 82

4.82 Gráficos das distâncias de Mahalanobis do modelo linear misto com intercepto aleatório na simulação com estrutura AR-1. . . . . . . . . . . . . . . . . 83

4.83 Gráficos dos resíduos do modelo marginal normal com estrutura AR-1 na simulação com estrutura AR-1. . . . . . . . . . . . . . . . . . 83

4.84 Gráficos dos resíduos do modelo marginal normal com estrutura uniforme na simulação com estrutura AR-1. . . . . . . . . . . . . . . . . . 84

5.1 Gráfico do volume do ejaculado (em $m l$ ) para os suínos. . . . . . . . . . . . . 88

5.2 Resíduos marginais e condicionais padronizados versus os índices das unidades experimentais - modelo 1. . . . . . . . . . . . . . . . . 91

5.3 Resíduos marginais e condicionais padronizados versus os valores ajustados modelo $1 . \ldots \ldots \ldots \ldots$. . . . . . . . . . . . . . . . 92

5.4 Gráfico dos resíduos para a estrutura de covariância versus os índices das unidades experimentais - modelo 1. . . . . . . . . . . . . . . . . 92

5.5 Gráficos Q-Q dos resíduos marginais e condicionais padronizados - modelo 1. . . 93

5.6 Gráfico Q-Q dos resíduos minimamente confundidos - modelo 1. . . . . . . . . 93

5.7 Gráfico Q-Q dos resíduos $t_{i j}^{*}$ - modelo 1. . . . . . . . . . . . . . . 94

5.8 Gráfico Q-Q dos resíduos quantílicos - modelo 1. . . . . . . . . . . . . . . 94

5.9 Gráficos das distâncias de Mahalanobis - modelo 1. . . . . . . . . . . . . . 95

5.10 Gráfico das distâncias de Cook versus os índices das unidades experimentais modelo 1. . . . . . . . . . . . . . . . . . . . . . . . . 95

5.11 Resíduos marginais e condicionais padronizados versus os índices das unidades experimentais - modelo 2. . . . . . . . . . . . . . . . . . . 96

5.12 Resíduos marginais e condicionais padronizados versus os valores ajustados modelo 2. . . . . . . . . . . . . . . . . . . . . . . 96

5.13 Gráfico dos resíduos para a estrutura de covariância versus os índices das unidades experimentais - modelo 2. . . . . . . . . . . . . . . . . . . . . 97

5.14 Gráficos Q-Q dos resíduos marginais e condicionais padronizados - modelo 2. $\quad$. 97

5.15 Gráfico Q-Q dos resíduos minimamente confundidos - modelo 2. . . . . . . . . 98

5.16 Gráfico Q-Q dos resíduos $t_{i j}^{*}$ - modelo 2. . . . . . . . . . . . . . . 98

5.17 Gráfico Q-Q dos resíduos quantílicos - modelo 2. . . . . . . . . . . . . 99

5.18 Gráficos das distâncias de Mahalanobis - modelo 2. . . . . . . . . . . . . . . . . 99

5.19 Gráfico das distâncias de Cook versus os índices das unidades experimentais modelo 2. . . . . . . . . . . . . . . . . . . . . . . 100 
5.20 Resíduos marginais e condicionais padronizados versus os índices das unidades experimentais - modelo 3 . . . . . . . . . . . . . . . . . . . 100

5.21 Resíduos marginais e condicionais padronizados versus os valores ajustados modelo 3. . . . . . . . . . . . . . . . . . . . . . . . 101

5.22 Gráfico dos resíduos para a estrutura de covariância versus os índices das unidades experimentais - modelo 3 . . . . . . . . . . . . . . . . . . . . . . 101

5.23 Gráficos Q-Q dos resíduos marginais e condicionais padronizados - modelo 3. . . 102

5.24 Gráfico Q-Q dos resíduos minimamente confundidos - modelo 3. . . . . . . . . . . 102

5.25 Gráfico Q-Q dos resíduos $t_{i j}^{*}$ - modelo $3 \ldots \ldots$. . . . . . . . . 103

5.26 Gráfico Q-Q dos resíduos quantílicos - modelo 3. . . . . . . . . . . . . . . 103

5.27 Gráficos das distâncias de Mahalanobis - modelo 3. . . . . . . . . . . . . . . . . 104

5.28 Gráfico das distâncias de Cook versus os índices das unidades experimentais modelo 3. . . . . . . . . . . . . . . . . . . . . . . . . . . . . . . 104 


\section{Lista de Tabelas}

2.1 Exemplos de MLGHs. . . . . . . . . . . . . . . . . . . . . . . . 11

2.2 Gráficos de diagnóstico para o modelo linear misto. . . . . . . . . . . . . 18

2.3 Componentes do desvio. . . . . . . . . . . . . . . . . . . 21

4.1 Estimativas (erros padrões) dos parâmetros do modelo linear misto com intercepto aleatório - estudo $1 \ldots \ldots \ldots \ldots \ldots$

4.2 Estimativas (erros padrões) dos parâmetros do modelo marginal normal com estrutura de correlação uniforme - estudo $1 \ldots \ldots$. . . . . . . . . . . . 31

4.3 Estimativas (erros padrões) dos parâmetros do modelo linear misto com inter-

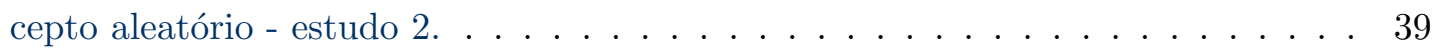

4.4 Estimativas (erros padrões) dos parâmetros do modelo marginal normal com estrutura de correlação uniforme - estudo $2 \ldots$. . . . . . . . . . . . . . . 40

4.5 Estimativas (erros padrões) dos parâmetros do modelo linear misto com intercepto aleatório - estudo $3 \ldots \ldots \ldots \ldots$. . . . . . . . . . . . 4 48

4.6 Estimativas (erros padrões) dos parâmetros do modelo marginal normal com estrutura de correlação uniforme - estudo $3 \ldots \ldots$. . . . . . . . . . . . . . . 49

4.7 Estimativas (erros padrões) dos parâmetros do modelo marginal gama com estrutura de correlação uniforme - estudo $3 \ldots$. . . . . . . . . . . . . . . . . . 49

4.8 Estimativas (erros padrões) dos parâmetros do modelo linear generalizado hierárquico gama com intercepto aleatório - estudo $3 . \ldots \ldots$. . . . . . . . 50

4.9 Estimativas (erros padrões) dos parâmetros do modelo com intercepto aleatório para a simulação com inclinação aleatória. . . . . . . . . . . . . . . . . . 61

4.10 Estimativas (erros padrões) dos parâmetros do modelo com intercepto e inclinação

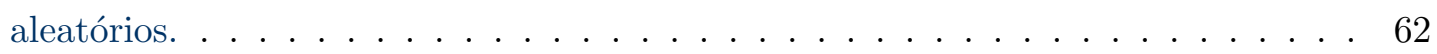

4.11 Estimativas (erros padrões) dos parâmetros do modelo marginal normal com matriz de correlações não estruturada. . . . . . . . . . . . . . . . . . . 62

4.12 Estimativas (erros padrões) dos parâmetros do modelo linear misto na simulação com estrutura AR-1. . . . . . . . . . . . . . . . . . 77

4.13 Estimativas (erros padrões) dos parâmetros do modelo marginal normal com estrutura AR-1 na simulação com estrutura AR-1. . . . . . . . . . . . . 78

4.14 Estimativas (erros padrões) dos parâmetros do modelo marginal normal com estrutura uniforme na simulação com estrutura AR-1. . . . . . . . . . . . . . 78

5.1 Volume do ejaculado $($ em $m l)$ para os suínos. . . . . . . . . . . . . 87

5.2 Estimativas (erros padrões) dos parâmetros - modelo 1. . . . . . . . . . . . . 89 
5.3 Estimativas (erros padrões) dos parâmetros - modelo $2 . \quad$. . . . . . . . . . . . . . 90

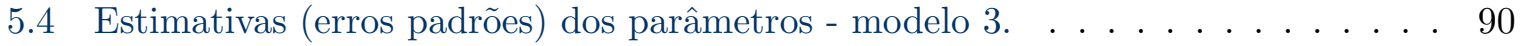

5.5 Comparação entre os três modelos ajustados. . . . . . . . . . . . . . . . . . . 91 


\section{Capítulo 1}

\section{Introdução}

Modelos lineares são ferramentas muito utilizadas nas mais diversas áreas de pesquisa. Primeiramente, foram definidos os modelos normais lineares, que representam o caso mais simples, sendo recomendados nas situações nas quais as variáveis respostas têm distribuição normal e as observações são independentes. Porém, muitas vezes a distribuição normal pode não ser a mais adequada para modelar os dados. Aproveitando o grande desenvolvimento dos aspectos computacionais, foram propostos os modelos lineares generalizados (MLGs), que permitem que a variável resposta pertença à família exponencial de distribuições, ainda trabalhando com observações independentes [McCullagh e Nelder, 1989].

Em diversas situações práticas, são frequentes estudos que consideram mais de uma observação da variável resposta para cada unidade experimental, o que caracteriza um experimento com medidas repetidas. Nessa situação, pode haver dependência entre observações de uma mesma unidade experimental, o que indica que os modelos lineares generalizados podem não ser a melhor solução para a análise dos dados.

Dentre as possíveis soluções propostas para trabalhar com observações correlacionadas, as principais abordagens utilizadas são os modelos condicionais e os modelos marginais.

Os modelos condicionais são os modelos com efeitos aleatórios ou modelos mistos, nos quais são incluídas variáveis latentes associadas a cada unidade experimental para modelar a estrutura de correlação. Os modelos condicionais são geralmente ajustados pelo método da máxima verossimilhança, sendo definidos condicionalmente aos efeitos aleatórios, o que permite a inferência sobre respostas individuais. Essa classe de modelos foi primeiramente desenvolvida para a distribuição normal, com os modelos lineares mistos (MLMs) [Laird e Ware, 1982] e expandida para toda a família exponencial de distribuições, com os modelos lineares generalizados mistos (MLGMs) [Breslow e Clayton, 1993], que permitem a inclusão de efeitos aleatórios gaussianos. Outra extensão proposta é a dos modelos lineares generalizados hierárquicos (MLGHs) [Lee e Nelder, 1996], que permitem outras possibilidades para a distribuição dos efeitos aleatórios, além de apresentar uma metodologia de estimação baseada em uma função de verossimilhança diferente daquela utilizada nos MLGMs.

Os modelos marginais são construídos para descrever as médias marginais diretamente, e impõem uma estrutura para a correlação das observações sem a utilização de efeitos aleatórios. O principal método de estimação para esse tipo de modelo é por meio das equações de estimação generalizadas (GEE - Generalized Estimating Equations), definidas por Liang e Zeger [1986].

As duas abordagens consideradas são diferentes, cada uma possuindo suas vantagens e des- 
vantagens. A escolha sobre qual delas utilizar muitas vezes é subjetiva, sendo motivada pela preferência do pesquisador. Com o objetivo de facilitar essa escolha, alguns autores têm abordado essa discussão como tema.

Diggle et al. [2002] recomendam o uso de acordo com o objetivo do estudo, sendo os modelos condicionais recomendados quando se tem interesse nas respostas individuais. Segundo Lee e Nelder [2004], cada modelo condicional leva a um modelo marginal específico e, além das estimativas individuais, os modelos condicionais permitem a obtenção das estimativas marginais. Além disso, podem ser obtidos resultados inválidos quando se ignora algum efeito aleatório, como o efeito de interação entre o componente aleatório e algum tratamento, por exemplo. Os usuários de modelos marginais não têm como checar tais afirmações. Essas propriedades motivam esses autores a recomendarem os modelos condicionais.

A favor dos modelos marginais, Jiang [2007] afirma que o método GEE para a estimação em modelos marginais é mais atrativo computacionalmente e não requer uma especificação total da distribuição dos dados. McCulloch et al. [2008] afirmam que erros na especificação do modelo podem resultar em estimadores viesados em modelos condicionais, enquanto as estimativas no modelo marginal são consistentes, mesmo que a estrutura de correlação seja escolhida de maneira equivocada. Os modelos condicionais permitem inferências individuais, mas envolvem mais suposições, assim como um ajuste mais complexo com apelo computacional intensivo. Hubbard et al. [2010] comentam que os modelos condicionais envolvem mais suposições, e essas são mais específicas, sendo mais prováveis de equívoco na prática.

Twisk [2004] compara as duas metodologias, considerando variáveis respostas contínuas e dicotômicas, assim como dados faltantes. Na análise com resposta contínua, os modelos ajustados por GEE com matrizes de correlação uniforme e não estruturada apresentam estimativas dos efeitos fixos muito próximas daquelas obtidas nos modelos mistos com apenas o intercepto aleatório e também com inclinação aleatória associada ao tempo. O mesmo também ocorreu na análise com dados faltantes. Para a variável resposta dicotômica, as estimativas apresentaram diferenças maiores de acordo com a abordagem utilizada.

As técnicas de diagnóstico estão completamente definidas para os modelos com observações independentes [Paula, 2012]. Para dados correlacionados, diversas técnicas têm sido propostas para a análise de diagnóstico em modelos lineares mistos [Nobre e Singer, 2007, 2011] e nos modelos baseados nas equações de estimação generalizadas [Venezuela et al., 2007]. Porém, as técnicas disponíveis para modelos lineares generalizados com efeitos aleatórios ainda não estão completamente estabelecidas.

Na prática, são comuns estudos analisados por meio de modelos condicionais sem a realização de análise de diagnóstico, ume vez que não há muitas rotinas computacionais para isso. O principal objetivo deste trabalho é apresentar e avaliar as técnicas de diagnóstico disponíveis para os modelos condicionais e marginais. Em especial para os modelos condicionais, diferentes propostas para o diagnóstico são sugeridas e implementadas.

No Capítulo 2, são apresentados os modelos condicionais: modelos lineares mistos (MLM), modelos lineares generalizados mistos (MLGMs) e modelos lineares generalizados hierárquicos (MLGHs), assim como as respectivas técnicas de diagnóstico. No Capítulo 3, são apresentados os modelos marginais, com o método de estimação via equações de estimação generalizadas (GEE) e as técnicas de diagnóstico. No Capítulo 4, são apresentados resultados de estudos de simulação, 
nos quais se procura avaliar as técnicas de diagnóstico apresentadas nos capítulos anteriores. Um exemplo de aplicação em um conjunto de dados reais está apresentado no Capítulo 5. Por fim, o Capítulo 6 contém as conclusões do trabalho, assim como sugestões para pesquisas futuras. 


\section{Capítulo 2}

\section{Modelos Condicionais}

Este capítulo apresenta os modelos condicionais. Por serem compostos por efeitos fixos e aleatórios, esses modelos também são conhecidos como modelos mistos. Primeiramente, serão apresentados alguns conceitos básicos dos modelos lineares mistos (MLMs), que representam um caso particular dos modelos lineares generalizados mistos (MLGMs) e dos modelos lineares generalizados hierárquicos (MLGHs), que serão apresentados na sequência. Além disso, serão abordadas algumas técnicas de diagnóstico para esses modelos.

\section{$2.1 \quad$ Modelos lineares mistos}

Os modelos lineares mistos constituem o caso mais simples de modelos com efeitos aleatórios. No caso de um experimento com medidas repetidas envolvendo $n$ unidades experimentais, denotamos por $n_{i}$ o número de observações da $i$-ésima unidade experimental, $i=1, \ldots, n$. Dessa forma, o total de observações é dado por $N=\sum_{i=1}^{n} n_{i}$. É definido o vetor de respostas

$$
\boldsymbol{y}=\left[\begin{array}{c}
\boldsymbol{y}_{1} \\
\vdots \\
\boldsymbol{y}_{n}
\end{array}\right]
$$

de dimensão $N$, em que $\boldsymbol{y}_{i}^{\top}=\left(y_{i 1}, \ldots, y_{i n_{i}}\right)$, sendo $y_{i j}$ o valor associado à $j$-ésima observação da $i$-ésima unidade experimental, com $j=1, \ldots, n_{i}$. O modelo linear misto é definido como

$$
\boldsymbol{y}_{i}=\boldsymbol{X}_{i} \boldsymbol{\beta}+\boldsymbol{Z}_{i} \boldsymbol{u}_{i}+\boldsymbol{\varepsilon}_{i}
$$

em que $\boldsymbol{X}_{i}$ são matrizes de posto completo de dimensão $n_{i} \times p$, associadas aos parâmetros dos efeitos fixos $\boldsymbol{\beta}=\left(\beta_{1}, \ldots, \beta_{p}\right)^{\top}$, e $\boldsymbol{Z}_{i}$ são matrizes de posto completo de dimensão $n_{i} \times q$, associadas aos efeitos aleatórios $\boldsymbol{u}_{i}=\left(u_{i 1}, \ldots, u_{i q}\right)^{\top}$, com o valor $q$ representando o número de efeitos aleatórios por unidade experimental. Além disso, será considerada a distribuição normal para a resposta e para os efeitos aleatórios, ou seja, $\boldsymbol{\varepsilon}_{i} \sim N\left(\mathbf{0}, \boldsymbol{\Sigma}_{i}\right)$ e $\boldsymbol{u}_{i} \sim N(\mathbf{0}, \boldsymbol{G})$ independentes. Assim,

$$
\boldsymbol{y}_{i} \sim N\left(\boldsymbol{X}_{i} \boldsymbol{\beta}, \boldsymbol{V}_{i}\right),
$$


em que

$$
\boldsymbol{V}_{i}=\boldsymbol{Z}_{i} \boldsymbol{G} \boldsymbol{Z}_{i}^{\top}+\boldsymbol{\Sigma}_{i}
$$

Condicionando nos efeitos aleatórios, temos

$$
\begin{aligned}
\mathrm{E}\left[\boldsymbol{y}_{i} \mid \boldsymbol{u}_{i}\right] & =\boldsymbol{\mu}_{i}=\boldsymbol{X}_{i} \boldsymbol{\beta}+\boldsymbol{Z}_{i} \boldsymbol{u}_{i}, \\
\operatorname{Var}\left[\boldsymbol{y}_{i} \mid \boldsymbol{u}_{i}\right] & =\boldsymbol{\Sigma}_{i},
\end{aligned}
$$

e

$$
\boldsymbol{y}_{i} \mid \boldsymbol{u}_{i} \sim N\left(\boldsymbol{X}_{i} \boldsymbol{\beta}+\boldsymbol{Z}_{i} \boldsymbol{u}_{i}, \boldsymbol{\Sigma}_{i}\right)
$$

Defindo as matrizes $\boldsymbol{X}=\left(\boldsymbol{X}_{1}^{\top}, \ldots, \boldsymbol{X}_{n}^{\top}\right)^{\top}, \boldsymbol{Z}=\operatorname{diag}\left(\boldsymbol{Z}_{1}, \ldots, \boldsymbol{Z}_{n}\right), \boldsymbol{V}=\operatorname{diag}\left(\boldsymbol{V}_{1}, \ldots, \boldsymbol{V}_{n}\right)$, $\boldsymbol{\Sigma}=\operatorname{diag}\left(\boldsymbol{\Sigma}_{1}, \ldots, \boldsymbol{\Sigma}_{n}\right)$ e $\boldsymbol{D}=\boldsymbol{I}_{n} \otimes \boldsymbol{G}$, em que $\otimes$ representa o produto de Kronecker [Harville, 2008], assim como os vetores $\boldsymbol{u}=\left(\boldsymbol{u}_{1}^{\top}, \ldots, \boldsymbol{u}_{n}^{\top}\right)^{\top}$ e $\boldsymbol{\varepsilon}=\left(\boldsymbol{\varepsilon}_{1}^{\top}, \ldots, \boldsymbol{\varepsilon}_{n}^{\top}\right)^{\top}$, o modelo pode ser escrito como

$$
\boldsymbol{y}=\boldsymbol{X} \boldsymbol{\beta}+\boldsymbol{Z u}+\varepsilon
$$

com $\varepsilon \sim N(\mathbf{0}, \boldsymbol{\Sigma})$ e $\boldsymbol{u} \sim N(\mathbf{0}, \boldsymbol{D})$ independentes. Nesse caso, temos

$$
\boldsymbol{y} \sim N(\boldsymbol{X} \boldsymbol{\beta}, \boldsymbol{V})
$$

em que

$$
\boldsymbol{V}=\boldsymbol{Z} \boldsymbol{D} Z^{\top}+\boldsymbol{\Sigma}
$$

e

$$
\boldsymbol{y} \mid \boldsymbol{u} \sim N(\boldsymbol{X} \boldsymbol{\beta}+\boldsymbol{Z} \boldsymbol{u}, \boldsymbol{\Sigma}) .
$$

Seja $\boldsymbol{\theta}$ o conjunto de parâmetros associados aos componentes da variância do modelo. Dessa forma, o vetor $\boldsymbol{\theta}$ é constituído pelos parâmetros associados à variância dos efeitos aleatórios, que definem a matriz $\boldsymbol{D}$, e pelos parâmetros associados à variância de $\boldsymbol{\varepsilon}$, que definem a matriz $\boldsymbol{\Sigma}$. Para diminuir o número de parâmetros de $\boldsymbol{\theta}$, estruturas de covariâncias podem ser definidas para as matrizes $\boldsymbol{G}$ e $\boldsymbol{\Sigma}$. Para a matriz $\boldsymbol{\Sigma}$, o caso mais utilizado é aquele que considera homocedasticidade e independência condicionais, ou seja, temos que as matrizes $\boldsymbol{\Sigma}_{i}$ são da forma $\boldsymbol{\Sigma}_{i}=\sigma^{2} \boldsymbol{I}_{n_{i}}$. Para a matriz $\boldsymbol{G}$, em geral não é definida nenhuma estrutura ou os efeitos aleatórios são considerados independentes, $\operatorname{com} \boldsymbol{G}$ sendo uma matriz diagonal com $q$ parâmetros. Assim, em um modelo com apenas o intercepto aleatório e uma única covariável $x_{i j}$, temos

$$
y_{i j}=\beta_{1}+u_{i}+\beta_{2} x_{i j}+\varepsilon_{i j}
$$

com $u_{i} \sim N\left(0, \sigma_{u}^{2}\right)$ e $\varepsilon_{i j} \sim N\left(0, \sigma^{2}\right)$. Assim, temos $\boldsymbol{\theta}=\left(\sigma^{2}, \sigma_{u}^{2}\right)^{\top}$. Nesse modelo, as matrizes 
$\boldsymbol{V}_{i}=\boldsymbol{Z}_{i} \boldsymbol{G} \boldsymbol{Z}_{i}^{\top}+\boldsymbol{\Sigma}_{i}$, com $\boldsymbol{Z}_{i}=\mathbf{1}_{n_{i}}$ para $i=1, \ldots, n$, assumem a estrutura uniforme, dada por

$$
\boldsymbol{V}_{i}=\left[\begin{array}{cccc}
\sigma_{u}^{2}+\sigma^{2} & \sigma_{u}^{2} & \cdots & \sigma_{u}^{2} \\
\sigma_{u}^{2} & \sigma_{u}^{2}+\sigma^{2} & \cdots & \sigma_{u}^{2} \\
\vdots & \vdots & \ddots & \vdots \\
\sigma_{u}^{2} & \sigma_{u}^{2} & \cdots & \sigma_{u}^{2}+\sigma^{2}
\end{array}\right]
$$

Nesse caso, para $j^{\prime} \neq j$, o coeficiente de correlação intraclasse é dado por

$$
\operatorname{Cor}\left[y_{i j}, y_{i j^{\prime}}\right]=\frac{\sigma_{u}^{2}}{\sigma_{u}^{2}+\sigma^{2}}
$$

No caso de um modelo com intercepto aleatório $u_{1 i} \sim N\left(0, \sigma_{u 1}^{2}\right)$ e inclinação aleatória $u_{2 i} \sim N\left(0, \sigma_{u 2}^{2}\right)$ associada a uma única covariável $x_{i j}$, temos

$$
y_{i j}=\beta_{1}+u_{1 i}+\left(\beta_{2}+u_{2 i}\right) x_{i j}+\varepsilon_{i j}
$$

com $u_{1 i} \sim N\left(0, \sigma_{u 1}^{2}\right), u_{2 i} \sim N\left(0, \sigma_{u 2}^{2}\right)$ e $\varepsilon_{i j} \sim N\left(0, \sigma^{2}\right)$ independentes. Considerando $\boldsymbol{u}_{i}=$ $\left(u_{1 i}, u_{2 i}\right)^{\top} \sim N(\mathbf{0}, \boldsymbol{G})$ e supondo que os efeitos aleatórios são independentes entre si, a matriz $\boldsymbol{G}$ é da forma

$$
\boldsymbol{G}=\left[\begin{array}{cc}
\sigma_{u 1}^{2} & 0 \\
0 & \sigma_{u 2}^{2}
\end{array}\right]
$$

Para esse modelo, temos

$$
\boldsymbol{Z}_{i}=\boldsymbol{X}_{i}=\left[\begin{array}{cc}
1 & x_{i 1} \\
\vdots & \vdots \\
1 & x_{i n_{i}}
\end{array}\right]
$$

e, consequentemente, as matrizes de covariâncias $\boldsymbol{V}_{i}$ são não estruturadas, sendo dadas por

$$
\boldsymbol{V}_{i}=\left[\begin{array}{cccc}
x_{i 1}^{2} \sigma_{u 2}^{2}+\sigma_{u 1}^{2}+\sigma^{2} & x_{i 1} x_{i 2} \sigma_{u 2}^{2}+\sigma_{u 1}^{2} & \cdots & x_{i 1} x_{i n_{i}} \sigma_{u 2}^{2}+\sigma_{u 1}^{2} \\
x_{i 1} x_{i 2} \sigma_{u 2}^{2}+\sigma_{u 1}^{2} & x_{i 2}^{2} \sigma_{u 2}^{2}+\sigma_{u 1}^{2}+\sigma^{2} & \cdots & x_{i 2} x_{i n_{i}} \sigma_{u 2}^{2}+\sigma_{u 1}^{2} \\
\vdots & \vdots & \ddots & \vdots \\
x_{i 1} x_{i n_{i}} \sigma_{u 2}^{2}+\sigma_{u 1}^{2} & x_{i 2} x_{i n_{i}} \sigma_{u 2}^{2}+\sigma_{u 1}^{2} & \cdots & x_{i n_{i}}^{2} \sigma_{u 2}^{2}+\sigma_{u 1}^{2}+\sigma^{2}
\end{array}\right]
$$

podendo assumir diversas formas dependendo dos valores das covariáveis $x_{i j}$ e, nos casos em que $x_{i j}$ assumem diferentes valores para cada observação, (2.4) resulta em um modelo heterocedástico. Nos casos em que $x_{i j}$ são variáveis dummies indicando grupos, as matrizes assumem estruturas iguais apenas para as observações pertencentes a um mesmo grupo. 


\subsubsection{Estimação em modelos lineares mistos}

O método de máxima verossimilhança é o mais utilizado na estimação dos parâmetros fixos $\boldsymbol{\beta}$. A função de verossimilhança associada ao modelo (2.1) é definida como

$$
L(\boldsymbol{\beta}, \boldsymbol{\theta})=\prod_{i=1}^{n}(2 \pi)^{-n_{i} / 2}\left|\boldsymbol{V}_{i}\right|^{1 / 2} \exp \left[-\frac{1}{2}\left(\boldsymbol{y}_{i}-\boldsymbol{X}_{i} \boldsymbol{\beta}\right)^{\top} \boldsymbol{V}_{i}^{-1}\left(\boldsymbol{y}_{i}-\boldsymbol{X}_{i} \boldsymbol{\beta}\right)\right],
$$

Assim, o logaritmo da verossimilhança é dado por

$$
l(\boldsymbol{\beta}, \boldsymbol{\theta})=-\frac{N}{2} \log 2 \pi-\frac{1}{2} \sum_{i=1}^{n} \log \left|\boldsymbol{V}_{i}\right|-\frac{1}{2} \sum_{i=1}^{n}\left(\boldsymbol{y}_{i}-\boldsymbol{X}_{i} \boldsymbol{\beta}\right)^{\top} \boldsymbol{V}_{i}^{-1}\left(\boldsymbol{y}_{i}-\boldsymbol{X}_{i} \boldsymbol{\beta}\right) .
$$

Para maximizar a função (2.5) com relação ao vetor de parâmetros $\boldsymbol{\beta}$, basta maximizar a quantidade

$$
Q(\boldsymbol{\beta})=\frac{1}{2} \sum_{i=1}^{n}\left(\boldsymbol{y}_{i}-\boldsymbol{X}_{i} \boldsymbol{\beta}\right)^{\top} \boldsymbol{V}_{i}^{-1}\left(\boldsymbol{y}_{i}-\boldsymbol{X}_{i} \boldsymbol{\beta}\right),
$$

o que é equivalente a utilizar o método de mínimos quadrados generalizados. Logo, considerando os parâmetros que definem a matriz $\boldsymbol{V}$ conhecidos, o estimador de $\boldsymbol{\beta}$ é dado por

$$
\hat{\boldsymbol{\beta}}=\left(\boldsymbol{X}^{\top} \boldsymbol{V}^{-1} \boldsymbol{X}\right)^{-1} \boldsymbol{X}^{\top} \boldsymbol{V}^{-1} \boldsymbol{y}
$$

que é o estimador linear não viesado de $\boldsymbol{\beta}$ com menor variância, sendo chamado de BLUE (Best Linear Unbiased Estimator). Dessa forma, a variância de $\hat{\boldsymbol{\beta}}$ é

$$
\operatorname{Var}(\hat{\boldsymbol{\beta}})=\left(\boldsymbol{X}^{\top} \boldsymbol{V}^{-1} \boldsymbol{X}\right)^{-1}
$$

Para os efeitos aleatórios, no caso em que todos os parâmetros são conhecidos, o melhor preditor linear não viesado, chamado de BLUP (Best Linear Unbiased Predictor), é a esperança condicional $\mathrm{E}[\boldsymbol{u} \mid \boldsymbol{y}]$. Considerando a distribuição conjunta

$$
\left[\begin{array}{l}
\boldsymbol{y} \\
\boldsymbol{u}
\end{array}\right] \sim N\left(\left[\begin{array}{c}
\boldsymbol{X} \boldsymbol{\beta} \\
\mathbf{0}
\end{array}\right],\left[\begin{array}{cc}
\boldsymbol{Z} & \boldsymbol{Z D} \\
\boldsymbol{D} \boldsymbol{Z}^{\top} & \boldsymbol{D}
\end{array}\right]\right)
$$

e a forma da distribuição condicional de partições da normal multivariada [Johnson e Wichern, 2007], obtemos

$$
\mathrm{E}[\boldsymbol{u} \mid \boldsymbol{y}]=\boldsymbol{D} \boldsymbol{Z}^{\top} \boldsymbol{V}^{-1}(\boldsymbol{y}-\boldsymbol{X} \boldsymbol{\beta})
$$

Henderson [1973] prova que, substituindo $\boldsymbol{\beta}$ em (2.7) pelo BLUE dado em (2.6), o preditor resultante é o melhor preditor linear não viesado para $\boldsymbol{u}$, dado por

$$
\hat{\boldsymbol{u}}=\boldsymbol{D} \boldsymbol{Z}^{\top} \boldsymbol{V}^{-1}(\boldsymbol{y}-\boldsymbol{X} \hat{\boldsymbol{\beta}})
$$

Quando não conhecemos os parâmetros $\boldsymbol{\theta}$ que definem $\boldsymbol{V}$, podemos substituí-los por esti- 
madores, gerando o BLUE empírico (EBLUE), dado por

$$
\hat{\boldsymbol{\beta}}=\left(\boldsymbol{X}^{\top} \hat{\boldsymbol{V}}^{-1} \boldsymbol{X}\right)^{-1} \boldsymbol{X}^{\top} \hat{\boldsymbol{V}}^{-1} \boldsymbol{y}
$$

e o BLUP empírico (EBLUP), dado por

$$
\hat{\boldsymbol{u}}=\hat{\boldsymbol{D}} \boldsymbol{Z}^{\top} \hat{\boldsymbol{V}}^{-1}(\boldsymbol{y}-\boldsymbol{X} \hat{\boldsymbol{\beta}}) .
$$

A variância do EBLUE pode ser estimada por

$$
\widehat{\operatorname{Var}}(\hat{\boldsymbol{\beta}})=\left(\boldsymbol{X}^{\top} \hat{\boldsymbol{V}}^{-1} \boldsymbol{X}\right)^{-1}
$$

As estimativas dos parâmetros de variância $\boldsymbol{\theta}$ também são obtidas por máxima verossimilhança. Porém, essas estimativas são viesadas. Para reduzir o viés desses estimadores relativos à estrutura de covariância, Patterson e Thompson [1971] propõem o método da máxima verossimilhança restrita, que consiste em maximizar a verossimilhança de uma transformação linear do vetor $\boldsymbol{y}$. Mais detalhes sobre a máxima verossimilhança restrita também podem ser obtidos em Demidenko [2004], por exemplo.

\subsection{Modelos lineares generalizados com efeitos aleatórios}

No contexto de modelos lineares generalizados com efeitos aleatórios, os elementos de $\boldsymbol{y}$ condicionados nos efeitos aleatórios, $y_{i j} \mid \boldsymbol{u}_{i}$, são considerados independentes e com a mesma forma de distribuição, que pode ser qualquer uma pertencente à família exponencial, ou seja, que pode ser escrita na forma

$$
f_{y_{i j} \mid \boldsymbol{u}_{i}}\left(y_{i j} \mid \boldsymbol{u}_{i}\right)=\exp \left\{\phi\left[y_{i j} \theta_{i j}-b\left(\theta_{i j}\right)\right]+c\left(y_{i j}, \phi\right)\right\},
$$

sendo $c(\cdot, \cdot)$ e $b(\cdot)$ funções diferenciáveis, $\theta_{i j}$ os parâmetros canônicos e $\phi^{-1}$ o parâmetro de dispersão. Na família exponencial, a média de $y_{i j} \mid \boldsymbol{u}_{i}$ e a função de variância são dadas, respectivamente, por $\mu_{i j}=\partial b\left(\theta_{i j}\right) / \partial \theta_{i j}$ e $V\left(\mu_{i j}\right)=\partial \mu_{i j} / \partial \theta_{i j}$.

\subsubsection{Modelos lineares generalizados mistos}

A partir da distribuição condicional definida em (2.11), são definidos os modelos lineares generalizados mistos (MLGMs), dados por

$$
\begin{aligned}
\mathrm{E}\left[y_{i j} \mid \boldsymbol{u}_{i}\right] & =\mu_{i j}=g^{-1}\left(\eta_{i j}\right), \\
\operatorname{Var}\left[y_{i j} \mid \boldsymbol{u}_{i}\right] & =\phi^{-1} V\left(\mu_{i j}\right),
\end{aligned}
$$

em que

$$
\eta_{i j}=\boldsymbol{x}_{i j}^{\top} \boldsymbol{\beta}+\boldsymbol{z}_{i j}^{\top} \boldsymbol{u}_{i}
$$

$g(\cdot)$ é a função de ligação, e $\boldsymbol{x}_{i j}^{\top}$ e $\boldsymbol{z}_{i j}^{\top}$ representam as $j$-ésimas linhas das matrizes $\boldsymbol{X}_{i}$ e $\boldsymbol{Z}_{i}$, respectivamente. Nesse caso, os efeitos aleatórios $\boldsymbol{u}_{1}, \ldots, \boldsymbol{u}_{n}$ são considerados independentes e 
identicamente distribuídos com distribuição de probabilidades dada por

$$
\boldsymbol{u}_{i} \sim N(\mathbf{0}, \boldsymbol{G})
$$

Utilizando propriedades da esperança condicional, obtemos

$$
\begin{aligned}
\mathrm{E}\left[y_{i j}\right] & =\mathrm{E}\left[\mathrm{E}\left[y_{i j} \mid \boldsymbol{u}_{i}\right]\right] \\
& =\mathrm{E}\left[g^{-1}\left(\boldsymbol{x}_{i j}^{\top} \boldsymbol{\beta}+\boldsymbol{z}_{i j}^{\top} \boldsymbol{u}_{i}\right)\right] .
\end{aligned}
$$

De maneira semelhante, é possível obter as variâncias

$$
\begin{aligned}
\operatorname{Var}\left[y_{i j}\right] & =\operatorname{Var}\left[\mathrm{E}\left[y_{i j} \mid \boldsymbol{u}_{i}\right]\right]+\mathrm{E}\left[\operatorname{Var}\left[y_{i j} \mid \boldsymbol{u}_{i}\right]\right] \\
& =\operatorname{Var}\left[g^{-1}\left(\boldsymbol{x}_{i j}^{\top} \boldsymbol{\beta}+\boldsymbol{z}_{i j}^{\top} \boldsymbol{u}_{i}\right)\right]+\mathrm{E}\left[\phi^{-1} V\left(g^{-1}\left(\boldsymbol{x}_{i j}^{\top} \boldsymbol{\beta}+\boldsymbol{z}_{i j}^{\top} \boldsymbol{u}_{i}\right)\right)\right]
\end{aligned}
$$

e as covariâncias marginais

$$
\begin{aligned}
\operatorname{Cov}\left[y_{i j}, y_{i j^{\prime}}\right] & =\operatorname{Cov}\left[\mathrm{E}\left[y_{i j} \mid \boldsymbol{u}_{i}\right], \mathrm{E}\left[y_{i j^{\prime}} \mid \boldsymbol{u}_{i}\right]\right]+\mathrm{E}\left[\operatorname{Cov}\left[y_{i j}, y_{i j^{\prime}} \mid \boldsymbol{u}_{i}\right]\right] \\
& =\operatorname{Cov}\left[g^{-1}\left(\boldsymbol{x}_{i j}^{\top} \boldsymbol{\beta}+\boldsymbol{z}_{i j}^{\top} \boldsymbol{u}_{i}\right), g^{-1}\left(\boldsymbol{x}_{i j^{\prime}}^{\top} \boldsymbol{\beta}+\boldsymbol{z}_{i j^{\prime}}^{\top} \boldsymbol{u}_{i}\right)\right]
\end{aligned}
$$

A estimação dos parâmetros dos efeitos fixos e de variância desses modelos é geralmente feita pelo método da máxima verossimilhança. A partir das definições (2.11) a (2.13), podemos escrever a função de verossimilhança marginal de $\boldsymbol{y}$ como

$$
L(\boldsymbol{\beta}, \boldsymbol{\theta})=\prod_{i=1}^{n} \int \prod_{j=1}^{n_{i}} f_{y_{i j} \mid \boldsymbol{u}_{i}}\left(y_{i j} \mid \boldsymbol{u}_{i}\right) f \boldsymbol{u}\left(\boldsymbol{u}_{i}\right) d \boldsymbol{u}_{i}
$$

Dessa forma, o logaritmo da função de verossimilhança é dado por

$$
\begin{aligned}
l(\boldsymbol{\beta}, \boldsymbol{\theta}) & =\log L(\boldsymbol{\beta}, \boldsymbol{\theta}) \\
& =\sum_{i=1}^{n} \log \int \prod_{j=1}^{n_{i}} f_{y_{i j} \mid \boldsymbol{u}_{i}}\left(y_{i j} \mid \boldsymbol{u}_{i}\right) f \boldsymbol{u}\left(\boldsymbol{u}_{i}\right) d \boldsymbol{u}_{i} .
\end{aligned}
$$

Nos casos em que a distribuição de $y_{i j} \mid \boldsymbol{u}_{i}$ é diferente da normal, as expressões apresentadas acima envolvem integrais que não podem ser resolvidas analiticamente. Dessa forma, os estimadores não podem ser expressos em forma fechada. Para resolver as integrais de maneira precisa, técnicas de aproximação são propostas. A aproximação de Laplace [Raudenbush et al., 2000] é bastante utilizada nessa situação. A quadratura de Gauss-Hermite [McCulloch et al., 2008] também pode ser utilizada. Essa técnica de integração numérica pode exigir computação intensiva, dependendo do número de componentes aleatórios. Outra possibilidade para a estimação dos modelos lineares generalizados mistos é o método da quase-verossimilhança penalizada [Breslow e Clayton, 1993].

\subsubsection{Modelos lineares generalizados hierárquicos}

Os modelos lineares generalizados hierárquicos (MLGHs), propostos por Lee e Nelder [1996], podem ser considerados como uma extensão dos modelos lineares generalizados mistos. A de- 
finição é semelhante, com $y_{i j} \mid \boldsymbol{u}_{i}$ seguindo distribuição da família exponencial dada em (2.11), com (2.12) e (2.13), com o preditor linear

$$
\eta_{i j}=\boldsymbol{x}_{i j}^{\top} \boldsymbol{\beta}+\boldsymbol{z}_{i j}^{\top} \boldsymbol{v}_{i}
$$

em que $\boldsymbol{v}_{i}=\left(v_{1}\left(u_{1 i}\right), \ldots, v_{q}\left(u_{q i}\right)\right)^{\top}$, sendo $v_{k}(\cdot)$ funções monótonas, para $i=1, \ldots, n$, e $k=$ $1, \ldots, q$. Os efeitos aleatórios $u_{i j}$ podem assumir diversas distribuições. A partir do conceito de distribuição conjugada, apresentado em Raiffa e Schlaifer [1961], os modelos que são compostos por essas distribuições são chamados de modelos lineares generalizados hierárquicos conjugados. Diversas combinações de distribuições e funções de ligação podem ser usadas para constituir um modelo linear generalizado hierárquico. Alguns exemplos de modelos utilizados estão resumidos na Tabela 2.1.

\begin{tabular}{|c|c|c|c|c|}
\hline$y \mid u$ & $g(\mu)$ & $u$ & $v(u)$ & Modelo \\
\hline Normal & $\mu$ & Normal & $u$ & $\begin{array}{l}\text { Modelo linear misto } \\
\text { (MLGH conjugado) }\end{array}$ \\
\hline Binomial & $\log \left(\frac{\mu}{1-\mu}\right)$ & Beta & $\log \left(\frac{u}{1-u}\right)$ & MLGH conjugado \\
\hline Binomial & $\log \left(\frac{\mu}{1-\mu}\right)$ & Normal & $u$ & MLGM Binomial \\
\hline Binomial & $\log (-\log (1-\mu))$ & Gama & $\log u$ & MLGH \\
\hline Gama & $\frac{1}{\mu}$ & Gama Inversa & $\frac{1}{u}$ & MLGH conjugado \\
\hline Gama & $\log \mu$ & Gama Inversa & $\frac{1}{u}$ & MLGH conjugado \\
\hline Gama & $\log \mu$ & Gama & $\log u$ & MLGH \\
\hline Poisson & $\log \mu$ & Normal & $u$ & MLGM Poisson \\
\hline Poisson & $\log \mu$ & Gama & $\log u$ & MLGH \\
\hline
\end{tabular}

Tabela 2.1: Exemplos de MLGHs.

Para a estimação dos parâmetros desses modelos foi desenvolvido um método baseado na verossimilhança-h, que é um caso particular de verossimilhança estendida [Bjørnstad, 1996]. Esse método é simples e computacionalmente atrativo, pois não há a necessidade de cálculo de integrais de grande ordem.

A verossimilhança-h é definida como

$$
h=l_{0}(\boldsymbol{\beta}, \phi ; \boldsymbol{y} \mid \boldsymbol{v})+l_{1}(\boldsymbol{\rho} ; \boldsymbol{v})
$$

em que $l_{0}(\boldsymbol{\beta}, \phi ; \boldsymbol{y} \mid \boldsymbol{v})$ e $l_{1}(\boldsymbol{\rho} ; \boldsymbol{v})$ são as funções de log-verossimilhança de $\boldsymbol{y} \mid \boldsymbol{v}$ e $\boldsymbol{v}$, respectivamente, com $\rho$ representando os parâmetros associados à distribuição dos efeitos aleatórios. Assim, a função pode ser escrita como

$$
h(\boldsymbol{\beta}, \boldsymbol{v}, \phi, \boldsymbol{\rho})=\sum_{i=1}^{n} \sum_{j=1}^{n_{i}} \log f_{y_{i j} \mid \boldsymbol{v}_{i}}\left(y_{i j} \mid \boldsymbol{v}_{i}\right)+\log f \boldsymbol{v}_{i}\left(\boldsymbol{v}_{i}\right),
$$

e deve ser maximizada com relação a $\boldsymbol{\beta}$ e $\boldsymbol{v}$ para a estimação dos efeitos fixos e aleatórios. Dessa forma, os valores preditos dos efeitos aleatórios são obtidos conjuntamente com as estimativas dos efeitos fixos. Os parâmetros associados à variância são obtidos a partir da verossimilhança-h 
perfilada ajustada [Lee e Nelder, 2001], que é uma generalização do método de máxima verossimilhança restrita utilizado na estimação dos parâmetros associados à variância do modelo linear misto.

No contexto de modelos lineares generalizados hierárquicos, a estimação pode ser vista como a estimação de um modelo linear generalizado aumentado, com variável resposta $\boldsymbol{y}_{a}=$ $\left(\boldsymbol{y}^{\top}, \boldsymbol{\psi}^{\top}\right)^{\top}$. Considerando o caso em que temos apenas um efeito aleatório por unidade experimental, temos

$$
\begin{aligned}
\mathrm{E}\left[y_{i j}\right] & =\mu_{i j} \\
\operatorname{Var}\left[y_{i j}\right] & =\phi^{-1} V\left(\mu_{i j}\right) \\
\mathrm{E}\left[\psi_{i}\right] & =u_{i} \\
\operatorname{Var}\left[\psi_{i}\right] & =\rho V_{M}\left(u_{i}\right),
\end{aligned}
$$

sendo $\boldsymbol{\psi}=\left(\psi_{1}, \ldots, \psi_{n}\right)^{\top}$ determinado de acordo com a restrição de $\mathrm{E}[\boldsymbol{u}]$, e com $V_{M}(\boldsymbol{u})$ sendo a função de variância associada à distribuição de $\boldsymbol{u}$. Dessa forma, sendo $\boldsymbol{\eta}=\boldsymbol{X} \boldsymbol{\beta}+\boldsymbol{Z} \boldsymbol{v}$ e $\boldsymbol{\eta}_{M}=\boldsymbol{v}$, o preditor linear é dado por

$$
\boldsymbol{\eta}_{a}=\left[\begin{array}{c}
\boldsymbol{\eta} \\
\boldsymbol{\eta}_{M}
\end{array}\right]=\boldsymbol{T}_{a} \boldsymbol{\omega}
$$

com a matriz do modelo aumentado

$$
\boldsymbol{T}_{a}=\left[\begin{array}{cc}
\boldsymbol{X} & \boldsymbol{Z} \\
\mathbf{0} & \boldsymbol{I}_{n}
\end{array}\right],
$$

e vetor de parâmetros

$$
\omega=\left[\begin{array}{l}
\beta \\
v
\end{array}\right]
$$

Consequentemente, dados os parâmetros de dispersão $\phi^{-1}$ e $\rho$, a estimativa de $\boldsymbol{\omega}$ pode ser obtida pelo processo iterativo de mínimos quadrados ponderados

$$
\boldsymbol{\omega}^{(m+1)}=\left[\left(\boldsymbol{T}_{a}^{\top} \boldsymbol{\Sigma}_{a}^{-1} \boldsymbol{T}_{a}\right)^{-1} \boldsymbol{T}_{a}^{\top} \boldsymbol{\Sigma}_{a}^{-1} \boldsymbol{z}_{a}\right]^{(m)},
$$

em que $\boldsymbol{z}_{a}=\left(\boldsymbol{z}^{\top}, \boldsymbol{z}_{M}^{\top}\right)^{\top}$, sendo $\boldsymbol{z}$ o vetor formado pelos valores

$$
z_{i j}=\eta_{i j}+\left(y_{i j}-\mu_{i j}\right)\left(\frac{\partial \eta_{i j}}{\partial \mu_{i j}}\right)
$$

associados a cada observação $y_{i j}$, e $\boldsymbol{z}_{M}$ formado pelos valores

$$
z_{M i}=v_{i}+\left(\psi_{i}-u_{i}\right)\left(\frac{\partial v_{i}}{\partial u_{i}}\right)
$$


associados aos componentes de $\boldsymbol{\psi}$. Além disso, a matriz $\boldsymbol{\Sigma}_{a}$ é definida como $\boldsymbol{\Sigma}_{a}=\boldsymbol{\Gamma}_{a} \boldsymbol{W}_{a}^{-1}$, com

$$
\boldsymbol{\Gamma}_{a}=\left[\begin{array}{cc}
\boldsymbol{\Phi}^{-1} & \mathbf{0} \\
\mathbf{0} & \boldsymbol{R}_{a}
\end{array}\right]
$$

sendo $\boldsymbol{\Phi}^{-1}$ a matriz diagonal de ordem $N$ formada pelos parâmetros de dispersão $\phi^{-1}$ da distribuição associada à resposta e $\boldsymbol{R}_{a}$ a matriz diagonal de ordem $n$ formada pelos parâmetros de dispersão $\rho$ da distribuição dos efeitos aleatórios. A matriz de pesos $\boldsymbol{W}_{a}$ é definida como

$$
\boldsymbol{W}_{a}=\left[\begin{array}{cc}
\boldsymbol{W} & \mathbf{0} \\
\mathbf{0} & \boldsymbol{W}_{M}
\end{array}\right]
$$

em que $\boldsymbol{W}$ é uma matriz diagonal de ordem $N$ formada pelos elementos

$$
w_{i j}=\left(\frac{\partial \mu_{i j}}{\partial \eta_{i j}}\right)^{2} V\left(\mu_{i j}\right)^{-1}
$$

e $\boldsymbol{W}_{M}$ é uma matriz diagonal de ordem $n$ formada pelos elementos

$$
w_{M i}=\left(\frac{\partial u_{i}}{\partial v_{i}}\right)^{2} V_{M}\left(u_{i}\right)^{-1}
$$

Mais detalhes sobre os modelos lineares generalizados hierárquicos podem ser vistos em Lee et al. [2006].

\subsection{Técnicas de diagnóstico}

As técnicas de diagnóstico são utilizadas para verificar a validade das suposições feitas para um modelo estatístico. Em modelos condicionais, existe maior variedade de técnicas de diagnóstico para os modelos lineares mistos.

\subsubsection{Diagnóstico em modelos lineares mistos}

Nobre e Singer [2007] é um exemplo de trabalho que mostra as técnicas de diagnóstico para o modelo linear misto. Considerando o modelo dado em (2.1), são definidos três tipos de erros:

- Erros marginais, definidos como $\boldsymbol{\xi}=\boldsymbol{y}-\mathrm{E}[\boldsymbol{y}]=\boldsymbol{y}-\boldsymbol{X} \boldsymbol{\beta}$;

- Erros condicionais, definidos como $\boldsymbol{\varepsilon}=\boldsymbol{y}-\mathrm{E}[\boldsymbol{y} \mid \boldsymbol{u}]=\boldsymbol{y}-\boldsymbol{X} \boldsymbol{\beta}-\boldsymbol{Z} \boldsymbol{u}$;

- Efeitos aleatórios, dados por $\boldsymbol{Z} \boldsymbol{u}=\mathrm{E}[\boldsymbol{y} \mid \boldsymbol{u}]-\mathrm{E}[\boldsymbol{y}]$.

Dessa forma, podemos definir os resíduos associados a cada tipo de erro: os resíduos marginais $\hat{\boldsymbol{\xi}}=\boldsymbol{y}-\boldsymbol{X} \hat{\boldsymbol{\beta}}$, condicionais $\hat{\boldsymbol{\varepsilon}}=\boldsymbol{y}-\boldsymbol{X} \hat{\boldsymbol{\beta}}-\boldsymbol{Z} \hat{\boldsymbol{u}}$ e os valores preditos dos efeitos aleatórios $\boldsymbol{Z} \hat{\boldsymbol{u}}$. Cada um desses três tipos de resíduos pode ser útil para avaliar diferentes suposições do modelo. 


\section{Resíduos marginais}

Definindo a matriz simétrica $\boldsymbol{Q}=\boldsymbol{V}^{-1}\left(\boldsymbol{I}_{N}-\boldsymbol{X}\left(\boldsymbol{X}^{\top} \boldsymbol{V}^{-1} \boldsymbol{X}\right)^{-1} \boldsymbol{X}^{\top} \boldsymbol{V}^{-1}\right)$, tal que $\boldsymbol{Q} \boldsymbol{V} \boldsymbol{Q}=\boldsymbol{Q}$ e $\boldsymbol{Q} \boldsymbol{X}=\mathbf{0}$, e utilizando as expressões de $\hat{\boldsymbol{\beta}}$ e $\hat{\boldsymbol{u}}$ dadas em (2.8) e (2.9), podemos escrever

$$
\hat{\boldsymbol{\xi}}=\boldsymbol{y}-\boldsymbol{X}\left(\boldsymbol{X}^{\top} \hat{\boldsymbol{V}}^{-1} \boldsymbol{X}\right)^{-1} \boldsymbol{X}^{\top} \hat{\boldsymbol{V}}^{-1} \boldsymbol{y}=\hat{\boldsymbol{V}} \hat{\boldsymbol{Q}} \boldsymbol{y}
$$

A partir dessa expressão, a variância de $\hat{\boldsymbol{\xi}}=\left(\hat{\boldsymbol{\xi}}_{1}, \ldots, \hat{\boldsymbol{\xi}}_{n}\right)^{\top}$ pode ser estimada por $\widehat{\operatorname{Var}}[\hat{\boldsymbol{\xi}}]=\hat{\boldsymbol{V}} \hat{\boldsymbol{Q}} \hat{\boldsymbol{V}}$. Os valores $q_{i i}$ da diagonal da matriz $\hat{\boldsymbol{V}} \hat{\boldsymbol{Q}} \hat{\boldsymbol{V}}$ podem ser utilizados para obter os resíduos marginais padronizados, dados por

$$
\hat{\boldsymbol{\xi}}_{i}^{*}=\frac{\hat{\boldsymbol{\xi}}_{i}}{\sqrt{q_{i i}}}
$$

O gráfico dos elementos de $\hat{\boldsymbol{\xi}}^{*}$ contra os valores ajustados marginais, definidos como $\hat{\boldsymbol{y}}_{m}=\boldsymbol{X} \hat{\boldsymbol{\beta}}$, pode ser empregado para checar a linearidade de $\boldsymbol{y}$ com respeito aos efeitos fixos. No caso em que a linearidade é válida, espera-se que os pontos estejam espalhados de maneira homogênea ao redor do valor zero. Um gráfico dos valores de $\hat{\boldsymbol{\xi}}^{*}$ contra os índices das observações é útil para detectar valores discrepantes. O gráfico Q-Q dos elementos de $\hat{\xi}^{*}$ também pode ser utilizado para checar a normalidade dos erros marginais.

Outra utilidade desse tipo de resíduo é checar a validade da estrutura de covariância $\boldsymbol{V}_{i}=$ $\boldsymbol{Z}_{i} \boldsymbol{G} \boldsymbol{Z}_{i}^{\top}+\boldsymbol{\Sigma}_{i}$. Lesaffre e Verbeke [1998] consideram a medida $\mathcal{V}_{i}=\left\|\boldsymbol{I}_{n_{i}}-\boldsymbol{\mathcal { R }}_{i} \boldsymbol{\mathcal { R }}_{i}^{\top}\right\|_{F}^{2}$, na qual $\boldsymbol{\mathcal { R }}_{i}=\hat{\boldsymbol{V}}_{i}^{-1 / 2} \hat{\boldsymbol{\xi}}_{i}$ e $\|\boldsymbol{A}\|_{F}$ denota a norma de Frobenius da matriz $\boldsymbol{A}$ [Graybill, 1983]. Quando o vetor de médias para o $i$-ésima unidade experimental é corretamente modelado por $\boldsymbol{X}_{i} \boldsymbol{\beta}$, a variância de $\boldsymbol{y}_{i}$ pode ser estimada por $\hat{\boldsymbol{\xi}}_{i} \hat{\boldsymbol{\xi}}_{i}^{\top}$. Nesse caso, em um gráfico de $\mathcal{V}_{i}$ contra os índices de unidades experimentais, espera-se que os valores fiquem próximos de zero. Esse gráfico pode ser utilizado para detectar situações em que a estrutura de covariância adotada não está adequada para alguma unidade experimental.

\section{Resíduos condicionais}

Os resíduos condicionais, dados por $\hat{\boldsymbol{\varepsilon}}=\boldsymbol{y}-\boldsymbol{X} \hat{\boldsymbol{\beta}}-\boldsymbol{Z} \hat{\boldsymbol{u}}$, podem ser utilizados para avaliar a homocedasticidade e a normalidade do erro condicional, sendo úteis também na detecção de observações discrepantes. Definindo a matriz

$$
\boldsymbol{C}=\boldsymbol{D}^{-1}+\boldsymbol{Z}^{\top} \boldsymbol{\Sigma}^{-1} \boldsymbol{Z}
$$

podemos escrever

$$
\begin{aligned}
\boldsymbol{V}^{-1} & =\left(\boldsymbol{\Sigma}+\boldsymbol{Z} \boldsymbol{D} \boldsymbol{Z}^{\top}\right)^{-1} \\
& =\boldsymbol{\Sigma}^{-1}-\boldsymbol{\Sigma}^{-1} \boldsymbol{Z} \boldsymbol{C}^{-1} \boldsymbol{Z}^{\top} \boldsymbol{\Sigma}^{-1} \\
& =\boldsymbol{\Sigma}^{-1}\left(\boldsymbol{I}_{N}-\boldsymbol{Z} \boldsymbol{C}^{-1} \boldsymbol{Z}^{\top} \boldsymbol{\Sigma}^{-1}\right)
\end{aligned}
$$


Assim, temos

$$
\begin{aligned}
\boldsymbol{D} \boldsymbol{Z}^{\top} \boldsymbol{V}^{-1} & =\boldsymbol{D} \boldsymbol{Z}^{\top}\left(\boldsymbol{\Sigma}^{-1}-\boldsymbol{\Sigma}^{-1} \boldsymbol{Z} \boldsymbol{C}^{-1} \boldsymbol{Z}^{\top} \boldsymbol{\Sigma}^{-1}\right) \\
& =\boldsymbol{D}\left(\boldsymbol{C}-\boldsymbol{Z}^{\top} \boldsymbol{\Sigma}^{-1} \boldsymbol{Z}\right) \boldsymbol{C}^{-1} \boldsymbol{Z}^{\top} \boldsymbol{\Sigma}^{-1} \\
& =\boldsymbol{C}^{-1} \boldsymbol{Z}^{\top} \boldsymbol{\Sigma}^{-1}
\end{aligned}
$$

A partir de (2.17), (2.16) e (2.18), e utilizando os valores de EBLUE e EBLUP, os resíduos condicionais podem ser escritos como

$$
\begin{aligned}
\hat{\varepsilon} & =\boldsymbol{y}-\boldsymbol{X} \hat{\boldsymbol{\beta}}-\boldsymbol{Z} \hat{\boldsymbol{D}} \boldsymbol{Z}^{\top} \hat{\boldsymbol{V}}^{-1}(\boldsymbol{y}-\boldsymbol{X} \hat{\boldsymbol{\beta}}) \\
& =\boldsymbol{y}-\boldsymbol{X} \hat{\boldsymbol{\beta}}-\boldsymbol{Z} \hat{\boldsymbol{C}} \boldsymbol{Z}^{\top} \hat{\boldsymbol{\Sigma}}^{-1}(\boldsymbol{y}-\boldsymbol{X} \hat{\boldsymbol{\beta}}) \\
& =\left(\boldsymbol{I}_{N}-\boldsymbol{Z} \boldsymbol{C}^{-1} \boldsymbol{Z}^{\top} \boldsymbol{\Sigma}^{-1}\right)(\boldsymbol{y}-\boldsymbol{X} \hat{\boldsymbol{\beta}}) \\
& =\hat{\boldsymbol{\Sigma}} \hat{\boldsymbol{V}}^{-1}(\boldsymbol{y}-\boldsymbol{X} \hat{\boldsymbol{\beta}}) .
\end{aligned}
$$

Substituindo (2.15) em (2.19), obtemos

$$
\hat{\varepsilon}=\hat{\Sigma} \hat{Q} y
$$

Logo, a variância de $\hat{\varepsilon}$ é estimada por

$$
\widehat{\operatorname{Var}}(\hat{\varepsilon})=\hat{\Sigma} \hat{\boldsymbol{Q}} \hat{\boldsymbol{\Sigma}}
$$

o que significa que os elementos de $\hat{\varepsilon}=\left(\hat{\varepsilon}_{1}, \ldots, \hat{\varepsilon}_{n}\right)^{\top}$ podem ter variâncias distintas. Dessa forma, sugere-se que os resíduos condicionais sejam padronizados. Nesse caso, o $i$-ésimo componente do vetor de resíduos condicionais padronizados é dado por

$$
\hat{\varepsilon}_{i}^{*}=\frac{\hat{\varepsilon}_{i}}{\sqrt{p_{i i}}}
$$

com $p_{i i}$ representando o $i$-ésimo elemento da diagonal principal da matriz $\hat{\boldsymbol{\Sigma}} \hat{\boldsymbol{Q}} \hat{\boldsymbol{\Sigma}}$, para $i=$ $1, \ldots, n$.

Para checar a homocedasticidade dos erros condicionais, é sugerido o gráfico dos elementos de $\hat{\boldsymbol{\varepsilon}}^{*}$ contra os valores ajustados $\hat{\boldsymbol{y}}=\boldsymbol{X} \hat{\boldsymbol{\beta}}+\boldsymbol{Z} \hat{\boldsymbol{u}}$. O gráfico dos resíduos condicionais padronizados contra os índices das observações pode ser utilizado para detectar observações discrepantes. Além disso, esses resíduos podem ser utilizados para verificar se os erros condicionais satisfazem a suposição de normalidade, através do gráfico do tipo Q-Q.

Hilden-Minton [1995] define que um resíduo é considerado puro para um específico tipo de erro se ele depende apenas dos componentes fixos e do erro que ele supostamente deve predizer. Os resíduos que dependem de outro tipo de erro são chamados de resíduos confundidos. Podemos escrever os três resíduos nas formas

$$
\begin{aligned}
\hat{\boldsymbol{\xi}} & =\left[\boldsymbol{I}_{N}-\boldsymbol{X}\left(\boldsymbol{X}^{\top} \boldsymbol{M} \boldsymbol{X}\right)^{-1} \boldsymbol{X}^{\top} \boldsymbol{M}\right] \boldsymbol{y} \\
& =\left[\boldsymbol{I}_{N}-\boldsymbol{X}\left(\boldsymbol{X}^{\top} \boldsymbol{M} \boldsymbol{X}\right)^{-1} \boldsymbol{X}^{\top} \boldsymbol{M}\right] \boldsymbol{X} \boldsymbol{\beta} \\
& =\left[\boldsymbol{I}_{N}-\boldsymbol{X}\left(\boldsymbol{X}^{\top} \boldsymbol{M} \boldsymbol{X}\right)^{-1} \boldsymbol{X}^{\top} \boldsymbol{M}\right] \boldsymbol{\xi},
\end{aligned}
$$




$$
\begin{aligned}
\hat{\varepsilon} & =\boldsymbol{\Sigma} \boldsymbol{Q}(\boldsymbol{X} \boldsymbol{\beta}+\boldsymbol{Z u}+\boldsymbol{\varepsilon}) \\
& =\boldsymbol{\Sigma} \boldsymbol{Q} \boldsymbol{\varepsilon}+\boldsymbol{\Sigma} \boldsymbol{Q} \boldsymbol{Z} \boldsymbol{u}
\end{aligned}
$$

$\mathrm{e}$

$$
\begin{aligned}
\boldsymbol{Z} \hat{\boldsymbol{u}} & =\boldsymbol{Z} \boldsymbol{D} \boldsymbol{Z}^{\top} \boldsymbol{Q}(\boldsymbol{X} \boldsymbol{\beta}+\boldsymbol{Z} \boldsymbol{u}+\boldsymbol{\varepsilon}) \\
& =\boldsymbol{Z} \boldsymbol{D} \boldsymbol{Z}^{\top} \boldsymbol{Q} \boldsymbol{Z} \boldsymbol{u}+\boldsymbol{Z} \boldsymbol{D} \boldsymbol{Z}^{\top} \boldsymbol{Q} \boldsymbol{\varepsilon}
\end{aligned}
$$

Segundo a definição de resíduos confundidos, pode-se notar que o resíduo marginal $\hat{\xi}$ não possui confundimento, enquanto o resíduo condicional $\hat{\varepsilon}$ é confundido com $\boldsymbol{u}$, e $\boldsymbol{Z} \hat{\boldsymbol{u}}$ é confundido com $\varepsilon$. Isso implica que $\hat{\varepsilon}$ pode não ser adequado para checar a normalidade de $\varepsilon$. Por esse motivo, o autor recomenda que é preciso tomar cuidado com a questão do confundimento quando for checada a normalidade do erro condicional.

Para diminuir esse problema, é definida uma quantidade que representa o efeito do confundimento no resíduo condicional. Hilden-Minton [1995] comenta que a capacidade de avaliar a normalidade de $\boldsymbol{\varepsilon}$ diminui quando $\operatorname{Var}\left[\boldsymbol{\Sigma} \boldsymbol{Q} \boldsymbol{Z}^{\top} \boldsymbol{u}\right]=\boldsymbol{\Sigma} \boldsymbol{Q} \boldsymbol{Z} \boldsymbol{D} \boldsymbol{Z}^{\top} \boldsymbol{Q} \boldsymbol{\Sigma}$ cresce em relação a $\operatorname{Var}[\boldsymbol{\Sigma} \boldsymbol{Q} \varepsilon]=\boldsymbol{\Sigma} \boldsymbol{Q} \boldsymbol{\Sigma} \boldsymbol{Q} \boldsymbol{\Sigma}$. Dessa forma, é definida a seguinte fração de confundimento para $\hat{\varepsilon}_{i}$ :

$$
0 \leq C F_{i}=\frac{\boldsymbol{U}_{i}^{\top} \boldsymbol{\Sigma} \boldsymbol{Q} \boldsymbol{Z} \boldsymbol{D} \boldsymbol{Z}^{\top} \boldsymbol{Q} \boldsymbol{\Sigma} \boldsymbol{U}_{i}}{\boldsymbol{U}_{i}^{\top} \boldsymbol{\Sigma} \boldsymbol{Q} \boldsymbol{\Sigma} \boldsymbol{Q} \boldsymbol{\Sigma} \boldsymbol{U}_{i}}=1-\frac{\boldsymbol{U}_{i}^{\top} \boldsymbol{\Sigma} \boldsymbol{Q} \boldsymbol{\Sigma} \boldsymbol{Q} \boldsymbol{\Sigma} \boldsymbol{U}_{i}}{\boldsymbol{U}_{i}^{\top} \boldsymbol{\Sigma} \boldsymbol{Q} \boldsymbol{\Sigma} \boldsymbol{U}_{i}} \leq 1,
$$

em que $\boldsymbol{U}_{i}$ representa a $i$-ésima coluna da matriz identidade $\boldsymbol{I}_{n}$. Assim, é sugerida uma transformação linear $\boldsymbol{L}^{\top} \hat{\varepsilon}$. Considerando as linhas $\boldsymbol{l}_{i}$ da matriz $\boldsymbol{L}$, devemos minimizar a fração de confundimento de $\boldsymbol{l}_{i}^{\top} \hat{\varepsilon}$. Isso significa maximizar a quantidade

$$
\kappa=\frac{l_{i}^{\top} \Sigma Q \Sigma Q \Sigma l_{i}}{l_{i}^{\top} \Sigma Q \Sigma l_{i}}
$$

considerando que $\operatorname{Var}\left[\boldsymbol{l}_{i}^{\top} \hat{\boldsymbol{\varepsilon}}\right] \propto \boldsymbol{l}_{i}^{\top} \boldsymbol{\Sigma} \boldsymbol{Q} \boldsymbol{\Sigma} \boldsymbol{l}_{i}>0$. Sabendo que $\boldsymbol{\Sigma}$ é uma matriz de posto completo, o interesse é o espaço não nulo da matriz $\boldsymbol{Q}$. Assumindo que $\boldsymbol{Q}$ é uma matriz $n \times n$ com posto $n-p$, através da decomposição do valor singular da matriz $\boldsymbol{\Sigma}^{1 / 2} \boldsymbol{Q} \boldsymbol{\Sigma}^{1 / 2}$ são obtidas as matrizes $\boldsymbol{K}$ e $\boldsymbol{\Lambda}$ que satisfazem

$$
\boldsymbol{\Sigma}^{1 / 2} \boldsymbol{Q} \boldsymbol{\Sigma}^{1 / 2}=\boldsymbol{K} \boldsymbol{\Lambda} \boldsymbol{K}^{\top}
$$

sendo $\boldsymbol{K}$ de dimensão $n \times(n-p)$ tal que $\boldsymbol{K}^{\top} \boldsymbol{K}=\boldsymbol{I}_{n-p}$ e $\boldsymbol{\Lambda}$ diagonal de ordem $n-p$ [Harville, 2008]. Considerando a quantidade $\boldsymbol{l}_{i}=\boldsymbol{\Sigma}^{-1 / 2} \boldsymbol{K} \boldsymbol{\Lambda}^{-1 / 2} \boldsymbol{b}$ para qualquer vetor $\boldsymbol{b}$ de dimensão $n-p$, a expressão (2.20) é escrita como

$$
\kappa=\frac{\boldsymbol{b}^{\top} \boldsymbol{\Lambda} \boldsymbol{b}}{\boldsymbol{b}^{\top} \boldsymbol{b}}
$$

Para a obtenção da transformação linear desejada, são considerados os vetores $\boldsymbol{b}=\boldsymbol{b}_{i}$, em que $\boldsymbol{b}_{i}$ representa a $i$-ésima coluna de $\boldsymbol{I}_{n-p}, i=1, \ldots, n-p$. Dessa forma, sendo $1 \geq \lambda_{1} \geq \lambda_{2} \geq$ $\cdots \geq \lambda_{n-p} \geq 0$ os valores da diagonal de $\boldsymbol{\Lambda}$ ordenados e $\boldsymbol{K}_{i}$ a $i$-ésima coluna de $\boldsymbol{K}$, temos

$$
\boldsymbol{l}_{i}=\boldsymbol{\Sigma}^{-1 / 2} \boldsymbol{K} \boldsymbol{\Lambda}^{-1 / 2} \boldsymbol{b}_{i}=\lambda_{i}^{-1 / 2} \boldsymbol{\Sigma}^{-1 / 2} \boldsymbol{K}_{i}
$$


Então, $\boldsymbol{l}_{i}^{\top} \boldsymbol{\varepsilon}=\lambda_{i}^{1 / 2} \boldsymbol{K}_{i}^{\top} \boldsymbol{\Sigma}^{-1 / 2} \boldsymbol{y}$, para $i=1, \ldots, n-p$, constitui uma sequência de erros homocedásticos não correlacionados com fração de confundimento igual a $1-\lambda_{i}$. Esses resíduos são denominados resíduos minimamente confundidos, e são utilizados para checar a normalidade do erro condicional por meio do gráfico Q-Q com envelope simulado.

Schützenmeister e Piepho [2011] comentam que a utilização de uma combinação linear dos resíduos dificulta a interpretação, pois cada elemento dos resíduos transformados não corresponde mais a cada observação individual do estudo. Outro problema citado é que uma combinação linear tende a aumentar o efeito de super normalidade, ou seja, os resíduos transformados podem ter distribuição mais próxima da normal do que os resíduos sem transformação [Atkinson, 1985]. Com isso, em algumas situações, o gráfico dos resíduos minimamente confundidos pode indicar normalidade, mesmo quando a distribuição dos erros condicionais não é normal. Para evitar esses problemas, os autores sugerem a utilização de um modelo linear, tratando os efeitos aleatórios como se fossem fixos. Essa abordagem consiste em considerar a matriz $\boldsymbol{X}^{*}$ e o vetor de parâmetros $\boldsymbol{\beta}^{*}$, modificados com a inclusão dos novos efeitos fixos. Em seguida, é ajustado o modelo normal linear

$$
\boldsymbol{y}=\boldsymbol{X}^{*} \boldsymbol{\beta}^{*}+\varepsilon
$$

$\operatorname{com} \varepsilon \sim N\left(\mathbf{0}, \sigma^{2} \boldsymbol{I}_{n}\right)$, e são obtidos os resíduos padronizados

$$
t_{i j}^{*}=\frac{\left(y_{i j}-\boldsymbol{x}_{i j}^{* \top} \hat{\boldsymbol{\beta}}^{*}\right)}{\hat{\sigma} \sqrt{\left(1-h_{i(j j)}^{*}\right)}},
$$

sendo $\boldsymbol{x}_{i j}^{* \top}$ a $j$-ésima linha da matriz $\boldsymbol{X}_{i}^{*}$, em que $\boldsymbol{X}^{*}=\left(\boldsymbol{X}_{1}^{* \top}, \ldots, \boldsymbol{X}_{n}^{* \top}\right)^{\top}$, e $h_{i(j j)}^{*}$ o $j$-ésimo elemento da diagonal do $i$-ésimo bloco diagonal da matriz de projeção $\boldsymbol{H}^{*}=\boldsymbol{X}^{*}\left(\boldsymbol{X}^{* \top} \boldsymbol{X}^{*}\right)^{-1} \boldsymbol{X}^{* \top}$, para $i=1, \ldots, n$ e $j=1, \ldots, n_{i}$. A proposta dos autores é a utilização do gráfico Q-Q desses resíduos para verificar a normalidade dos erros condicionais.

Outra possibilidade é considerar os resíduos quantílicos, definidos por Dunn e Smyth [1996]. Os resíduos quantílicos são dados pela expressão

$$
r_{i j}^{q}=\Phi^{-1}\left(F\left(y_{i j} ; \hat{\mu}_{i j}, \hat{\sigma}^{2}\right)\right)
$$

na qual $\Phi(\cdot)$ representa a função de distribuição acumulada da normal padrão e $F(\cdot)$ é a função de distribuição acumulada de $y_{i j} \mid \boldsymbol{u}$, que neste caso é $N\left(\mu_{i j}, \sigma^{2}\right)$. Um gráfico Q-Q desses resíduos para a distribuição normal é útil para verificar a validade do modelo ajustado.

\section{Valores preditos dos efeitos aleatórios}

A quantidade $\boldsymbol{Z}_{i} \hat{\boldsymbol{u}}_{i}$ representa a diferença entre o valor predito e o valor médio populacional predito para a $i$-ésima unidade experimental. Pinheiro e Bates [2000] recomendam o gráfico dos elementos de $\hat{\boldsymbol{u}}_{i}$ contra os índices associados às unidades experimentais, para identificar a presença de alguma unidade experimental discrepante. Porém, os valores de $\hat{\boldsymbol{u}}_{i}$ só são comparáveis quando as covariáveis $\boldsymbol{Z}_{i}$ são as mesmas para todas as unidades experimentais. Dessa forma, gráficos alternativos são propostos para detectar unidades experimentais discrepantes. 
Uma possibilidade é utilizar $\boldsymbol{Z}_{i} \hat{\boldsymbol{u}}_{i}$. Waternaux et al. [1989] propõem a utilização da distância de Mahalanobis

$$
\hat{\zeta}_{i}=\hat{\boldsymbol{u}}_{i}^{\top} \operatorname{Var}\left[\hat{\boldsymbol{u}}_{i}-\boldsymbol{u}_{i}\right]^{-1} \hat{\boldsymbol{u}}_{i}
$$

em que

$$
\operatorname{Var}\left[\hat{\boldsymbol{u}}_{i}-\boldsymbol{u}_{i}\right]=\boldsymbol{G}-\boldsymbol{G} \boldsymbol{Z}_{i}^{\top} \boldsymbol{V}_{i}^{-1} \boldsymbol{Z}_{i} \boldsymbol{G}+\boldsymbol{G} \boldsymbol{Z}_{i}^{\top} \boldsymbol{V}_{i}^{-1} \boldsymbol{X}_{i}\left(\boldsymbol{X}^{\top} \boldsymbol{V}^{-1} \boldsymbol{X}\right)^{-1} \boldsymbol{X}_{i}^{\top} \boldsymbol{V}_{i}^{-1} \boldsymbol{Z}_{i} \boldsymbol{G} .
$$

No caso de normalidade dos efeitos aleatórios, $\hat{\zeta}_{i}$ deve ter distribuição $\chi_{q}^{2}$. Dessa forma, podemos utilizar um gráfico do tipo Q-Q da distribuição $\chi_{q}^{2}$ para verificar se a distribuição dos efeitos aleatórios é normal. O gráfico dos valores $\hat{\zeta}_{i}$ contra os índices das unidades experimentais é utilizado para detectar unidades experimentais discrepantes.

De maneira semelhante ao que foi apresentado em Nobre [2004], os gráficos que podem ser utilizados no caso normal estão resumidos na Tabela 2.2.

\begin{tabular}{lll}
\hline Gráfico & Tipo de resíduo & Diagnóstico para \\
\hline$\hat{\boldsymbol{\xi}}_{i}$ vs. valores ajustados & marginal & Linearidade dos efeitos fixos \\
$\hat{\boldsymbol{\xi}}_{i}$ vs. índices das & marginal & Presença de observações discrepantes \\
unidades experimentais & & \\
$\mathcal{V}_{i}$ vs. índices das & marginal & Estruturas de covariância $\left(\boldsymbol{V}_{i}\right)$ \\
unidades experimentais & & \\
$\hat{\boldsymbol{\varepsilon}}_{i}^{*}$ vs. índices das & condicional & Presença de observações discrepantes \\
unidades experimentais & & \\
$\hat{\boldsymbol{\varepsilon}}_{i}^{*}$ vs. valores ajustados & condicional & Homocedasticidade dos erros condicionais \\
Q-Q para os resíduos & marginal & Normalidade dos erros marginais $\left(\boldsymbol{\xi}_{i}\right)$ \\
marginais padronizados & & \\
Q-Q para os resíduos & condicional & Normalidade dos erros condicionais $\left(\boldsymbol{\varepsilon}_{i}\right)$ \\
minimamente confundidos & & \\
Q-Q para os resíduos & condicional & Normalidade dos erros condicionais $\left(\boldsymbol{\varepsilon}_{i}\right)$ \\
condicionais padronizados & & \\
Q-Q para os resíduos & & Normalidade dos erros condicionais $\left(\boldsymbol{\varepsilon}_{i}\right)$ \\
quantílicos & & \\
Q-Q para $t_{i j}^{*}$ & & Normalidade dos erros condicionais $\left(\boldsymbol{\varepsilon}_{i}\right)$ \\
$\hat{\zeta}_{i}$ vs. índices das & valor predito & Presença de unidades \\
unidades experimentais & & experimentais discrepantes \\
Q-Q $\chi^{2}$ para $\hat{\zeta}_{i}$ & valor predito & Normalidade dos efeitos aleatórios $\left(\boldsymbol{u}_{i}\right)$ \\
\hline
\end{tabular}

Tabela 2.2: Gráficos de diagnóstico para o modelo linear misto.

\section{Influência}

Outra possibilidade no diagnóstico em modelos mistos está na detecção de pontos de alavanca e influentes. Demidenko e Stukel [2005] e Nobre e Singer [2011] estão entre as principais 
referências no assunto. Uma abordagem diferente pode ser encontrada em Gumedze et al. [2010]. Um ponto de alavanca é aquele que possui um valor diferente dos demais no que diz respeito às variáveis explicativas, podendo influenciar nos valores ajustados $\hat{\boldsymbol{y}}$. A medida que determina os pontos de alavanca é obtida da matriz de projeção, definida como $\boldsymbol{H}=\partial \hat{\boldsymbol{y}} / \partial \boldsymbol{y}^{\top}$. Valores altos em $\boldsymbol{H}$ indicam pontos de alavanca.

Nos modelos lineares mistos, Fung et al. [2002] definem a matriz de projeção marginal considerando os valores ajustados marginais $\hat{\boldsymbol{y}}_{m}=\boldsymbol{X} \hat{\boldsymbol{\beta}}$, dada por

$$
\boldsymbol{H}^{m}=\boldsymbol{X}\left(\boldsymbol{X}^{\top} \boldsymbol{V}^{-1} \boldsymbol{X}\right)^{-1} \boldsymbol{X}^{\top} \boldsymbol{V}^{-1}
$$

que é quadrada de ordem $N$. Sendo $\boldsymbol{H}_{i}^{m}$ o $i$-ésimo bloco diagonal da matriz $\boldsymbol{H}^{m}$, o $j$-ésimo elemento da diagonal de $\boldsymbol{H}_{i}^{m}$, denotado como $h_{i(j j)}^{m}$, é a medida de alavancagem associada à $j$-ésima observação da $i$-ésima unidade experimental. Assim, cada elemento da diagonal de $\boldsymbol{H}^{m}$ está associado a uma observação da amostra. O gráfico dos elementos da diagonal de $\boldsymbol{H}^{m}$ contra os índices das unidades experimentais pode ser utilizado para detectar pontos de alavanca. Existe também a possibilidade de utilização de outra definição da matriz de projeção. Considerando os valores ajustados $\hat{\boldsymbol{y}}=\boldsymbol{X} \hat{\boldsymbol{\beta}}+\boldsymbol{Z} \hat{\boldsymbol{u}}$, obtemos a matriz de projeção conjunta

$$
\boldsymbol{H}^{c}=\frac{\partial \hat{\boldsymbol{y}}}{\partial \boldsymbol{y}^{\top}}=\boldsymbol{X}\left(\boldsymbol{X}^{\top} \boldsymbol{V}^{-1} \boldsymbol{X}\right)^{-1} \boldsymbol{X}^{\top} \boldsymbol{V}^{-1}+\boldsymbol{Z} \boldsymbol{D} \boldsymbol{Z}^{\top} \boldsymbol{Q}
$$

que também incorpora as informações dos efeitos aleatórios [Nobre, 2004]. Analogamente ao que foi feito no caso da matriz $\boldsymbol{H}^{m}$, os elementos da diagonal de $\boldsymbol{H}^{c}$, denotados como $h_{i(j j)}^{c}$, são utilizados em gráfico contra os índices das unidades experimentais para detectar pontos de alavanca.

Os pontos influentes são aqueles que apresentam diferença no que diz respeito à variável resposta, podendo ter grande peso na estimação dos parâmetros do modelo. A principal medida utilizada para detectá-los é a distância de Cook, que consiste em uma medida do efeito que a exclusão de uma ou mais observações têm sobre as estimativas dos parâmetros. Nos modelos lineares mistos, a distância de Cook referente a um conjunto $I$ de observações é dada por

$$
C D_{I}=\frac{\left(\hat{\boldsymbol{\beta}}-\hat{\boldsymbol{\beta}}_{(I)}\right)^{\top}\left(\boldsymbol{X}^{\top} \boldsymbol{V}^{-1} \boldsymbol{X}\right)\left(\hat{\boldsymbol{\beta}}-\hat{\boldsymbol{\beta}}_{(I)}\right)}{p}=\frac{\left(\hat{\boldsymbol{y}}-\hat{\boldsymbol{y}}_{(I)}\right)^{\top} \boldsymbol{V}^{-1}\left(\hat{\boldsymbol{y}}-\hat{\boldsymbol{y}}_{(I)}\right)}{p},
$$

em que $\hat{\boldsymbol{\beta}}_{(I)}$ e $\hat{\boldsymbol{y}}_{(I)}$ são, respectivamente, o vetor de estimativas dos parâmetros fixos e o vetor de valores ajustados, ambos obtidos com a exclusão das observações de $I$. Em geral, calcula-se a medida para cada observação, dada por $C D_{i j}$. Quanto mais alto o valor de $C D_{i j}$, maior é a influência da $j$-ésima observação da $i$-ésima unidade experimental nas estimativas dos parâmetros fixos $\boldsymbol{\beta}$. Tan et al. [2001] comentam a limitação da medida (2.22) e sugerem a utilização da distância de Cook condicional, definida a partir do modelo

$$
\boldsymbol{y}=\boldsymbol{X}^{*} \boldsymbol{\beta}^{*}+\varepsilon
$$

no qual $\boldsymbol{X}^{*}=\left[\begin{array}{ll}\boldsymbol{X} & \boldsymbol{Z}\end{array}\right], \boldsymbol{\beta}^{*}=\left(\boldsymbol{\beta}^{\top}, \boldsymbol{u}^{\top}\right)^{\top}$ e $\operatorname{Var}[\boldsymbol{\varepsilon}]=\sigma^{2} \boldsymbol{I}_{N}$. A distância de Cook condicional é 
dada por

$$
C D_{i j}^{c o n d}=\sum_{k=1}^{n} \frac{\left(\hat{\boldsymbol{y}}_{k}-\hat{\boldsymbol{y}}_{k(i j)}\right)^{\top}\left(\hat{\boldsymbol{y}}_{k}-\hat{\boldsymbol{y}}_{k(i j)}\right)}{\sigma^{2}\{(n-1) q+p\}}
$$

em que $\hat{\boldsymbol{y}}_{k}=\boldsymbol{X}_{k} \hat{\boldsymbol{\beta}}+\boldsymbol{Z}_{k} \hat{\boldsymbol{u}}_{k}, \hat{\boldsymbol{y}}_{k(i j)}=\boldsymbol{X}_{k} \hat{\boldsymbol{\beta}}_{(i j)}+\boldsymbol{Z}_{k} \hat{\boldsymbol{u}}_{k(i j)}$ com $\hat{\boldsymbol{\beta}}_{(i j)}$ e $\hat{\boldsymbol{u}}_{k(i j)}$ os valores obtidos com a exclusão da $j$-ésima observação da $i$-ésima unidade experimental, para $k=1, \ldots, n$, $i=1, \ldots, n$ e $j=1, \ldots, n_{i}$. A medida (2.23) pode ser decomposta na forma

$$
C D_{i j}^{\text {cond }}=C D_{i j}^{\text {cond } 1}+C D_{i j}^{\text {cond } 2}+C D_{i j}^{\text {cond } 3}
$$

em que o primeiro termo é

$$
C D_{i j}^{c o n d 1}=\frac{\left(\hat{\boldsymbol{\beta}}-\hat{\boldsymbol{\beta}}_{(i j)}\right)^{\top} \boldsymbol{X}^{\top} \boldsymbol{X}\left(\hat{\boldsymbol{\beta}}-\hat{\boldsymbol{\beta}}_{(i j)}\right)}{\sigma^{2}\{(n-1) q+p\}},
$$

e pode ser interpretado como a medida de distância de Cook para os parâmetros associados aos efeitos fixos. O segundo termo é dado por

$$
C D_{i j}^{c o n d 2}=\sum_{k=1}^{n} \frac{\left(\hat{\boldsymbol{u}}_{k}-\hat{\boldsymbol{u}}_{k(i j)}\right)^{\top} \boldsymbol{Z}_{k}^{\top} \boldsymbol{Z}_{k}\left(\hat{\boldsymbol{u}}_{k}-\hat{\boldsymbol{u}}_{k(i j)}\right)}{\sigma^{2}\{(n-1) q+p\}},
$$

e pode ser interpretado como a medida de distância para os parâmetros associados aos efeitos aleatórios. O terceiro termo é

$$
C D_{i j}^{c o n d 3}=\frac{2\left(\hat{\boldsymbol{\beta}}-\hat{\boldsymbol{\beta}}_{(i j)}\right)^{\top} \sum_{k=1}^{n} \boldsymbol{X}_{k}^{\top} \boldsymbol{Z}_{k}\left(\hat{\boldsymbol{u}}_{k}-\hat{\boldsymbol{u}}_{k(i j)}\right)}{\sigma^{2}\{(n-1) q+p\}},
$$

que pode ser entendido como uma medida de covariância entre a mudança nas estimativas de $\boldsymbol{\beta}$ e $\boldsymbol{u}$. Para detectar observações influentes, podem ser utilizados os gráficos dos elementos $C D_{i j}$ ou de $C D_{i j}^{\text {cond }}$ contra os índices das unidades experimentais.

Sobre o conceito de influência local, não considerado neste trabalho, detalhes podem ser encontrados em Beckman et al. [1987] e Lesaffre e Verbeke [1998], por exemplo.

\subsubsection{Diagnóstico em modelos lineares generalizados mistos e hierárquicos}

Considerando o contexto de modelos lineares generalizados hierárquicos, a partir da expressão (2.14), podemos definir a matriz

$$
\boldsymbol{H}^{a}=\boldsymbol{T}_{a}\left(\boldsymbol{T}_{a}^{\top} \boldsymbol{\Sigma}_{a}^{-1} \boldsymbol{T}_{a}\right)^{-1} \boldsymbol{T}_{a}^{\top} \boldsymbol{\Sigma}_{a}^{-1}
$$

que exerce o papel de matriz de projeção. Da mesma forma que é feita no caso dos modelos lineares generalizados, os elementos $h_{i(j j)}^{a}$ da diagonal da matriz $\boldsymbol{H}^{a}$ podem ser úteis para o diagnóstico do modelo. Na diagonal da matriz $\boldsymbol{H}^{a}$, teremos $N+n$ elementos, sendo os $\mathrm{N}$ primeiros $h_{i(j j)}^{a}$ associados a cada observação e os $n$ últimos associados a cada unidade experimental. Gráficos dos elementos $h_{i(j j)}^{a}$ contra os índices das observações são utilizados para 
detectar pontos de alavanca. Para a detecção de unidades experimentais influentes, não há técnica disponível nos pacotes computacionais. Uma possibilidade que pode ser considerada é a proposta de Xiang et al. [2002].

Em geral, os resíduos utilizados nesse contexto são os componentes do desvio $d_{i j}$, baseados no conceito de função desvio, definida como

$$
D(\boldsymbol{y}, \hat{\boldsymbol{\mu}})=2\{l(\boldsymbol{y}, \boldsymbol{y})-l(\boldsymbol{y}, \hat{\boldsymbol{\mu}})\}=\phi \sum_{i=1}^{n} \sum_{j=1}^{n_{i}} d_{i j}^{2}
$$

A Tabela 2.3 apresenta as expressões que os componentes do desvio assumem, de acordo com a distribuição.

\begin{tabular}{|c|c|c|}
\hline Distribuição & $d_{i j}^{2}$ & \\
\hline Normal & $\left(y_{i j}-\hat{\mu}_{i j}\right)^{2}$ & \\
\hline Poisson & $\begin{array}{ll}2\left\{y_{i j} \log \left(y_{i j} / \hat{\mu}_{i j}\right)-\left(y_{i j}-\hat{\mu}_{i j}\right)\right\}, & \text { se } y_{i j}>0 \\
2 \mu_{i j}, & \text { se } y_{i j}=0\end{array}$ & \\
\hline Binomial & $\left\{\begin{array}{l}y_{i j} \log \left(\frac{y_{i j}}{n_{i j} \hat{\mu}_{i j}}\right)+\left(n_{i j}-y_{i j}\right) \log \left\{\frac{\left(1-y_{i j} / n_{i j}\right)}{\left(1-\mu_{i j}\right)}\right\} \\
-2 n_{i j} \log \left(1-\hat{\mu}_{i j}\right), \\
-2 n_{i j} \log \hat{\mu}_{i j},\end{array}\right.$ & $\begin{array}{l}\text { se } 0<y_{i j}<n_{i j} \\
\text { se } y_{i j}=0 \\
\text { se } y_{i j}=n_{i j}\end{array}$ \\
\hline Gama & $2\left\{-\log \left(y_{i j} / \hat{\mu}_{i j}\right)+\left(y_{i j}-\hat{\mu}_{i j}\right) / \hat{\mu}_{i j}\right\}$ & \\
\hline
\end{tabular}

Tabela 2.3: Componentes do desvio.

Para padronizar esses resíduos, devemos utilizar os $N$ primeiros elementos da diagonal da matriz estimada $\hat{\boldsymbol{H}}^{a}$, representados por $\hat{h}_{i(j j)}^{a}$, fazendo

$$
t_{i j}^{a}= \pm \frac{d_{i j}}{\sqrt{\hat{\phi}^{-1}\left(1-\hat{h}_{i(j j)}^{a}\right)}} .
$$

Gráficos dos componentes de desvio padronizados contra os índices das observações podem ser utilizados para detectar observações aberrantes. Outra possibilidade é a utilização desses resíduos para a verificação da suposição referente à distribuição condicional da resposta, por meio de gráficos Q-Q.

Para verificar a distribuição condicional da resposta, a proposta de Schützenmeister e Piepho [2011] também pode ser aplicada para outros modelos. Analogamente ao que foi feito em (2.21), pode ser ajustado um modelo linear generalizado considerando os efeitos aleatórios como se fossem efeitos fixos, utilizando novamente $\boldsymbol{X}^{*}$ e $\boldsymbol{\beta}^{*}$. Assim, é utilizado o gráfico Q-Q para os componentes do desvio padronizados

$$
t_{i j}^{*}= \pm \frac{d_{i j}^{*}}{\sqrt{\left(\hat{\phi}^{*}\right)^{-1}\left(1-\hat{h}_{i(j j)}^{*}\right)}},
$$

com $\hat{h}_{i(j j)}^{*}$ representando o $j$-ésimo elemento da diagonal do $i$-ésimo bloco diagonal da matriz de projeção $\hat{\boldsymbol{H}}^{*}=\hat{\boldsymbol{W}}^{1 / 2} \boldsymbol{X}^{*}\left(\boldsymbol{X}^{* \top} \hat{\boldsymbol{W}} \boldsymbol{X}^{*}\right)^{-1} \boldsymbol{X}^{* \top} \hat{\boldsymbol{W}}^{1 / 2}$, sendo $\boldsymbol{W}=\operatorname{diag}\left(\boldsymbol{W}_{1}, \ldots, \boldsymbol{W}_{n}\right)$, com $\boldsymbol{W}_{i}=\operatorname{diag}\left(w_{i 1}, \ldots, w_{i n_{i}}\right)$ e $w_{i j}=\left(\partial \mu_{i j} / \partial \eta_{i j}\right)^{2} V\left(\mu_{i j}\right)^{-1}$. 
O gráfico Q-Q dos resíduos quantílicos também pode ser utilizado para verificar a qualidade do ajuste, definidos nesse caso como

$$
r_{i j}^{q}=\Phi^{-1}\left(F\left(y_{i j} ; \hat{\mu}_{i j}, \hat{\phi}\right)\right),
$$

na qual $\Phi(\cdot)$ representa a função de distribuição acumulada da normal padrão, e $F(\cdot)$ é a função de distribuição acumulada da distribuição de $y_{i j} \mid \boldsymbol{u}$. 


\section{Capítulo 3}

\section{Modelos Marginais}

Neste capítulo será apresentado o modelo marginal para dados correlacionados, com foco na estimação por meio das equações de estimação generalizadas. Ao contrário da especificação condicional do modelo apresentada em (2.12), os modelos marginais trabalham diretamente com a médias marginais, e impõem uma estrutura de correlação sem a utilização de efeitos aleatórios. Nesse caso, as observações $y_{i j}$ seguem distribuição da família exponencial

$$
f_{y_{i j}}\left(y_{i j}\right)=\exp \left\{\phi\left[y_{i j} \theta_{i j}-b\left(\theta_{i j}\right)\right]+c\left(y_{i j}, \phi\right)\right\}
$$

com $c(\cdot, \cdot)$ e $b(\cdot)$ representando funções diferenciáveis, $\theta_{i j}$ são os parâmetros canônicos, e $\phi^{-1}$ representa o parâmetro de dispersão, de maneira semelhante ao que foi definido em (2.11). A partir da média $\mu_{i j}=\partial b\left(\theta_{i j}\right) / \partial \theta_{i j}$ e da função de variância $V\left(\mu_{i j}\right)=\partial \mu_{i j} / \partial \theta_{i j}$, o modelo é definido como

$$
\begin{aligned}
\mathrm{E}\left[y_{i j}\right] & =\mu_{i j}=g^{-1}\left(\eta_{i j}\right), \\
\operatorname{Var}\left[y_{i j}\right] & =\phi^{-1} V\left(\mu_{i j}\right),
\end{aligned}
$$

em que

$$
\eta_{i j}=\boldsymbol{x}_{i j}^{\top} \boldsymbol{\beta}
$$

sendo $x_{i j}^{\top}$ a $j$-ésima linha da matriz $\boldsymbol{X}_{i}$ de covariáveis associada à $i$-ésima unidade experimental, de dimensão $n_{i} \times p$.

\subsection{Equações de estimação generalizadas}

As equações de estimação generalizadas foram desenvolvidas por Liang e Zeger [1986] para a análise de dados provenientes de estudos com medidas repetidas. Definindo, para cada unidade experimental $i, \operatorname{com} i=1, \ldots, n$ e $j=1, \ldots, n_{i}$, as matrizes

$$
\boldsymbol{\Delta}_{i}=\operatorname{diag}\left(\frac{\partial \theta_{i j}}{\partial \eta_{i j}}\right)
$$


$\mathrm{e}$

$$
\boldsymbol{A}_{i}=\operatorname{diag}\left(\frac{\partial \mu_{i j}}{\partial \theta_{i j}}\right)=\operatorname{diag}\left(V\left(\mu_{i j}\right)\right),
$$

e considerando a matriz $\boldsymbol{R}_{i}$, que é a verdadeira matriz de correlações das observações referentes à $i$-ésima unidade experimental, a matriz de covariâncias é dada por

$$
\operatorname{Var}\left[\boldsymbol{y}_{i}\right]=\boldsymbol{\Omega}_{i}=\phi^{-1} \boldsymbol{A}_{i}^{1 / 2} \boldsymbol{R}_{i} \boldsymbol{A}_{i}^{1 / 2}
$$

Na prática, em geral $\boldsymbol{R}_{i}$ é desconhecida. Para contornar esse problema, assume-se que $\boldsymbol{R}_{i}=\boldsymbol{R}$, $i=1, \ldots, n$, e é definida a matriz $\boldsymbol{R}(\boldsymbol{\alpha})$, que é uma matriz que satisfaz as condições para ser uma matriz de correlações, e é conhecida como matriz de correlações de trabalho. O vetor $\boldsymbol{\alpha}$ é formado por parâmetros que caracterizam completamente essa matriz. Para diminuir o número de parâmetros no vetor $\boldsymbol{\alpha}$, podemos adotar diferentes estruturas para $\boldsymbol{R}(\boldsymbol{\alpha})$.

A seguir, são apresentados alguns exemplos de estruturas que podem ser adotadas para a matriz $\boldsymbol{R}(\boldsymbol{\alpha})$, considerando $n_{i}=4$ :

a) Independência

$$
\boldsymbol{R}(\boldsymbol{\alpha})=\left[\begin{array}{llll}
1 & 0 & 0 & 0 \\
0 & 1 & 0 & 0 \\
0 & 0 & 1 & 0 \\
0 & 0 & 0 & 1
\end{array}\right]
$$

b) Estrutura uniforme

$$
\boldsymbol{R}(\boldsymbol{\alpha})=\left[\begin{array}{cccc}
1 & \alpha & \alpha & \alpha \\
\alpha & 1 & \alpha & \alpha \\
\alpha & \alpha & 1 & \alpha \\
\alpha & \alpha & \alpha & 1
\end{array}\right]
$$

c) Estrutura auto-regressiva de ordem 1 (AR-1)

$$
\boldsymbol{R}(\boldsymbol{\alpha})=\left[\begin{array}{cccc}
1 & \alpha & \alpha^{2} & \alpha^{3} \\
\alpha & 1 & \alpha & \alpha^{2} \\
\alpha^{2} & \alpha & 1 & \alpha \\
\alpha^{3} & \alpha^{2} & \alpha & 1
\end{array}\right]
$$


d) Estrutura Toeplitz

$$
\boldsymbol{R}(\boldsymbol{\alpha})=\left[\begin{array}{cccc}
1 & \alpha_{1} & \alpha_{2} & \alpha_{3} \\
\alpha_{1} & 1 & \alpha_{1} & \alpha_{2} \\
\alpha_{2} & \alpha_{1} & 1 & \alpha_{1} \\
\alpha_{3} & \alpha_{2} & \alpha_{1} & 1
\end{array}\right]
$$

e) Não estruturada

$$
\boldsymbol{R}(\boldsymbol{\alpha})=\left[\begin{array}{cccc}
1 & \alpha_{12} & \alpha_{13} & \alpha_{14} \\
\alpha_{12} & 1 & \alpha_{23} & \alpha_{24} \\
\alpha_{13} & \alpha_{23} & 1 & \alpha_{34} \\
\alpha_{14} & \alpha_{24} & \alpha_{34} & 1
\end{array}\right]
$$

Definindo $\boldsymbol{D}_{i}^{* \top}=-\boldsymbol{X}_{i}^{\top} \boldsymbol{\Delta}_{i} \boldsymbol{A}_{i}$, a forma geral das equações de estimação generalizadas é dada por

$$
\boldsymbol{\Psi}(\boldsymbol{\beta})=\sum_{i=1}^{N} \boldsymbol{D}_{i}^{* \top} \boldsymbol{\Omega}_{i}^{-1}\left(\boldsymbol{y}_{i}-\boldsymbol{\mu}_{i}\right)=\mathbf{0}
$$

em que $\boldsymbol{\Omega}_{i}=\phi^{-1} \boldsymbol{A}_{i}^{1 / 2} \boldsymbol{R}(\boldsymbol{\alpha}) \boldsymbol{A}_{i}^{1 / 2}$.

Para obter o estimador $\hat{\boldsymbol{\beta}}_{G}$ de $\boldsymbol{\beta}$ por meio da equação (3.4), é utilizado um processo iterativo que combina o método scoring de Fisher para estimar $\boldsymbol{\beta}$ com o método dos momentos para estimar $\boldsymbol{\alpha}$ e $\phi$. Esse processo iterativo pode ser escrito na forma

$$
\hat{\boldsymbol{\beta}}_{G}^{(m+1)}=\left[\left(\boldsymbol{X}^{\top} \hat{\boldsymbol{W}} \boldsymbol{X}\right)^{-1}\left(\boldsymbol{X}^{\top} \hat{\boldsymbol{W}} \boldsymbol{z}^{*}\right)\right]^{(m)},
$$

$\operatorname{com} \boldsymbol{X}=\left(\boldsymbol{X}_{1}^{\top}, \ldots, \boldsymbol{X}_{n}^{\top}\right)^{\top}$ e $\hat{\boldsymbol{W}}=\operatorname{diag}\left(\hat{\boldsymbol{W}}_{1}, \ldots, \hat{\boldsymbol{W}}_{n}\right)$, em que

$$
\hat{\boldsymbol{W}}_{i}=\hat{\boldsymbol{\Delta}}_{i} \hat{\boldsymbol{A}}_{i} \hat{\boldsymbol{\Omega}}_{i}^{-1} \hat{\boldsymbol{A}}_{i} \hat{\boldsymbol{\Delta}}_{i}
$$

e $\boldsymbol{z}^{*}=\left(\boldsymbol{z}_{1}^{* \top}, \ldots, \boldsymbol{z}_{n}^{* \top}\right)^{\top}, \mathrm{com}$

$$
\boldsymbol{z}_{i}^{*}=\hat{\boldsymbol{\eta}}_{i}+\left(\hat{\boldsymbol{A}}_{i} \hat{\boldsymbol{\Delta}}_{i}\right)^{-1}\left(\boldsymbol{y}_{i}-\hat{\boldsymbol{\mu}}_{i}\right)
$$

Liang e Zeger [1986] demonstram que, sob algumas condições de regularidade e consistência dos estimadores de $\boldsymbol{\alpha}$ e $\phi$, o estimador $\hat{\boldsymbol{\beta}}_{G}$ é $\sqrt{n}$-consistente com relação a $\boldsymbol{\beta}$, e essa consistência é válida mesmo nos casos em que a estrutura de correlação utilizada não corresponde à verdadeira estrutura de correlação de $\boldsymbol{y}$. Além disso, $\hat{\boldsymbol{\beta}}_{G}$ é tal que

$$
\sqrt{n}\left(\hat{\boldsymbol{\beta}}_{G}-\boldsymbol{\beta}\right) \stackrel{D}{\longrightarrow} N_{p}\left(\mathbf{0}, \boldsymbol{J}_{G}^{-1}\right)
$$


com

$$
\boldsymbol{J}_{G}=\lim _{n \rightarrow \infty} \frac{\boldsymbol{J}_{n G}}{n}
$$

em que

$$
\boldsymbol{J}_{n G}=\left(\sum_{i=1}^{n} \boldsymbol{S}_{i}\right)\left(\sum_{i=1}^{n} \boldsymbol{V}_{i}^{*}\right)^{-1}\left(\sum_{i=1}^{n} \boldsymbol{S}_{i}\right)
$$

sendo $\boldsymbol{S}_{i}$ e $\boldsymbol{V}_{i}^{*}$ definidos como

$$
\begin{aligned}
\boldsymbol{S}_{i} & =\mathrm{E}_{\beta}\left[\frac{\partial}{\partial \boldsymbol{\beta}^{\top}} \boldsymbol{D}_{i}^{* \top} \boldsymbol{\Omega}_{i}^{-1}\left(\boldsymbol{y}_{i}-\boldsymbol{\mu}_{i}\right)\right] \\
& =\boldsymbol{D}_{i}^{* \top} \boldsymbol{\Omega}_{i}^{-1} \mathrm{E}_{\beta}\left[\frac{\partial}{\partial \boldsymbol{\beta}^{\top}}\left(\boldsymbol{y}_{i}-\boldsymbol{\mu}_{i}\right)\right] \\
& =-\boldsymbol{D}_{i}^{* \top} \boldsymbol{\Omega}_{i}^{-1} \boldsymbol{D}_{i}^{*},
\end{aligned}
$$

e

$$
\begin{aligned}
\boldsymbol{V}_{i}^{*} & =\mathrm{E}_{\beta}\left[\boldsymbol{D}_{i}^{* \top} \boldsymbol{\Omega}_{i}^{-1}\left(\boldsymbol{y}_{i}-\boldsymbol{\mu}_{i}\right)\left(\boldsymbol{y}_{i}-\boldsymbol{\mu}_{i}\right)^{\top} \boldsymbol{\Omega}_{i}^{-1} \boldsymbol{D}_{i}^{*}\right] \\
& =\boldsymbol{D}_{i}^{* \top} \boldsymbol{\Omega}_{i}^{-1} \operatorname{Var}\left[\boldsymbol{y}_{i}\right] \boldsymbol{\Omega}_{i}^{-1} \boldsymbol{D}_{i}^{*}
\end{aligned}
$$

A partir disso, duas abordagens são utilizadas para definir dois estimadores para a matriz de covariâncias de $\hat{\boldsymbol{\beta}}_{G}$. O primeiro estimador é chamado de ingênuo, naive ou model based, e é obtido considerando $\operatorname{Var}\left[\boldsymbol{y}_{i}\right]=\boldsymbol{\Omega}_{i}$ e, consequentemente,

$$
\boldsymbol{V}_{i}^{*}=\boldsymbol{D}_{i}^{* \top} \boldsymbol{\Omega}_{i}^{-1} \boldsymbol{D}_{i}^{*}
$$

Utilizando uma estimativa para $\boldsymbol{\Omega}_{i}$ e substituindo (3.7) em (3.6), o estimador naive é dado por

$$
\hat{\boldsymbol{J}}_{n G}^{-1}=\left(\sum_{i=1}^{n} \hat{\boldsymbol{D}}_{i}^{* \top} \hat{\boldsymbol{\Omega}}_{i}^{-1} \hat{\boldsymbol{D}}_{i}^{*}\right)^{-1}
$$

que é consistente quando a matriz de correlações de trabalho está corretamente especificada. O segundo estimador utilizado é o estimador conhecido como robusto, empírico ou sanduíche. Esse estimador é sempre consistente, sendo definido a partir da aproximação $\operatorname{Var}\left[\boldsymbol{y}_{i}\right] \simeq\left(\boldsymbol{y}_{i}-\right.$ $\left.\hat{\boldsymbol{\mu}}_{i}\right)\left(\boldsymbol{y}_{i}-\hat{\boldsymbol{\mu}}_{i}\right)^{\top}$, que resulta em

$$
\boldsymbol{V}_{i}=\boldsymbol{D}_{i}^{* \top} \boldsymbol{\Omega}_{i}^{-1}\left(\boldsymbol{y}_{i}-\hat{\boldsymbol{\mu}}_{i}\right)\left(\boldsymbol{y}_{i}-\hat{\boldsymbol{\mu}}_{i}\right)^{\top} \boldsymbol{\Omega}_{i}^{-1} \boldsymbol{D}_{i}^{*}
$$

O estimador robusto é dado por

$$
\hat{\boldsymbol{J}}_{n G}^{-1}=\left(\sum_{i=1}^{n} \hat{\boldsymbol{S}}_{i}\right)\left(\sum_{i=1}^{n} \hat{\boldsymbol{D}}_{i}^{* \top} \hat{\boldsymbol{\Omega}}_{i}^{-1}\left(\boldsymbol{y}_{i}-\hat{\boldsymbol{\mu}}_{i}\right)\left(\boldsymbol{y}_{i}-\hat{\boldsymbol{\mu}}_{i}\right)^{\top} \hat{\boldsymbol{\Omega}}_{i}^{-1} \hat{\boldsymbol{D}}_{i}^{*}\right)^{-1}\left(\sum_{i=1}^{n} \hat{\boldsymbol{S}}_{i}\right) .
$$

Na situação em que a matriz $\boldsymbol{R}(\boldsymbol{\alpha})$ é a verdadeira matriz de correlações dos $\boldsymbol{y}_{i}$, o estimador robusto e o estimador naive são iguais. 
A estimativa para o parâmetro de dispersão $\phi^{-1}$ pode ser obtida através dos resíduos de Pearson, definidos como

$$
r_{i j}=\frac{\left(y_{i j}-\hat{\mu}_{i j}\right)}{\sqrt{V\left(\hat{\mu}_{i j}\right)}}
$$

Um estimador consistente para $\phi^{-1}$ é

$$
\hat{\phi}^{-1}=\left(\sum_{i=1}^{n} \sum_{j=1}^{n_{i}} \frac{\left(r_{i j}\right)^{2}}{N-p}\right)^{-1} .
$$

As estimativas dos parâmetros $\boldsymbol{\alpha}$ vão depender da estrutura adotada. Considerando um estudo balanceado, ou seja, $n_{1}=\cdots=n_{n}=m$, quando $\boldsymbol{R}(\boldsymbol{\alpha})$ é não-estruturada (3.3), esta pode ser estimada como

$$
\hat{\boldsymbol{R}}=\frac{\sum_{i=1}^{n} \boldsymbol{r}_{i} \boldsymbol{r}_{i}^{\top}}{n-p}
$$

em que $\boldsymbol{r}_{i}=\left(r_{i 1}, \ldots, r_{i m}\right)^{\top}$. No caso da estrutura uniforme (3.1), uma estimativa de $\alpha$ é dada por

$$
\hat{\alpha}=\phi \frac{\sum_{i=1}^{n} \sum_{j>k} r_{i j} r_{i k}}{\sum_{i=1}^{n} \frac{1}{2} m(m-1)-p} .
$$

Para a estrutura auto-regressiva de ordem 1 (AR-1) (3.2), uma estimativa é

$$
\hat{\alpha}=\frac{\sum_{i=1}^{n} \sum_{j=1}^{m-1} r_{i j} r_{i, j+1}}{n(m-1)} .
$$

Mais detalhes sobre as possíveis estimativas podem ser encontrados em Artes e Botter [2005], por exemplo.

\subsection{Técnicas de diagnóstico}

Venezuela et al. [2007] apresentam as técnicas de diagnóstico para os modelos marginais. A partir do processo iterativo definido em (3.5), pode-se interpretar $\hat{\boldsymbol{\beta}}_{G}$ como a solução de mínimos quadrados da regressão normal linear com variável resposta $\hat{W}^{1 / 2} \boldsymbol{z}^{*}$ e matriz de planejamento $\hat{\boldsymbol{W}}^{1 / 2} \boldsymbol{X}$. A partir disso, considerando as matrizes $\hat{\boldsymbol{A}}=\operatorname{diag}\left(\hat{\boldsymbol{A}}_{1}, \ldots, \hat{\boldsymbol{A}}_{n}\right)$ e $\hat{\boldsymbol{\Delta}}=$ $\operatorname{diag}\left(\hat{\boldsymbol{\Delta}}_{1}, \ldots, \hat{\boldsymbol{\Delta}}_{n}\right)$, é definido o resíduo ordinário

$$
\boldsymbol{r}^{*}=\hat{\boldsymbol{W}}^{1 / 2}\left(\boldsymbol{z}^{*}-\hat{\boldsymbol{\eta}}\right)=\hat{\boldsymbol{W}}^{1 / 2}(\hat{\boldsymbol{A}} \hat{\boldsymbol{\Delta}})^{-1}(\boldsymbol{y}-\hat{\boldsymbol{\mu}}) .
$$

Assumindo que $\operatorname{Var}\left(\boldsymbol{z}^{*}\right)=(\hat{\boldsymbol{\Delta}} \hat{\boldsymbol{A}})^{-1} \operatorname{Var}(\boldsymbol{y})(\hat{\boldsymbol{A}} \hat{\boldsymbol{\Delta}})^{-1} \simeq \hat{\boldsymbol{W}}^{-1}$, podemos escrever $\operatorname{Var}\left(\boldsymbol{r}^{*}\right) \simeq\left(\boldsymbol{I}_{N}-\right.$ $\left.\boldsymbol{H}^{g}\right)$, sendo $\boldsymbol{H}^{g}=\operatorname{diag}\left(\boldsymbol{H}_{1}^{g}, \ldots, \boldsymbol{H}_{n}^{g}\right)$, tal que

$$
\boldsymbol{H}_{i}^{g}=\hat{\boldsymbol{W}}_{i}^{1 / 2} \boldsymbol{X}_{i}\left(\boldsymbol{X}^{\top} \hat{\boldsymbol{W}} \boldsymbol{X}\right)^{-1} \boldsymbol{X}_{i}^{\top} \hat{\boldsymbol{W}}_{i}^{1 / 2}
$$

Utilizando a matriz $\boldsymbol{H}^{g}$ para padronizar os resíduos, temos que cada elemento do vetor de 
resíduos padronizados é dado por

$$
r_{i j}^{p}=\frac{\boldsymbol{e}_{(i j)}^{\top} \hat{\boldsymbol{W}}_{i}^{1 / 2}\left(\hat{\boldsymbol{A}}_{i} \hat{\boldsymbol{\Delta}}_{i}\right)^{-1}\left(\boldsymbol{y}_{i}-\hat{\boldsymbol{\mu}}_{i}\right)}{\sqrt{1-h_{i(j j)}^{g}}}
$$

em que $\boldsymbol{e}_{(i j)}$ representa um vetor de tamanho $n_{i}$ com o valor 1 na $j$-ésima posição e 0 nas posições restantes, e $h_{i(j j)}^{g}$ representa o $j$-ésimo elemento da diagonal de $\boldsymbol{H}_{i}^{g}$, para $i=1, \ldots, n$, e $j=1, \ldots, n_{i}$. A análise gráfica dos resíduos padronizados $r_{i j}^{p}$ pode ser utilizada na detecção de observações aberrantes. Além disso, os resíduos padronizados podem ser utilizados para avaliar o ajuste do modelo, por meio de gráficos do tipo Q-Q com envelope simulado. Mais detalhes sobre a simulação de dados correlacionados podem ser vistos em Park e Shin [1998].

Escrevendo o resíduo ordinário na forma $\boldsymbol{r}^{*}=\left(\boldsymbol{I}_{N}-\boldsymbol{H}^{g}\right) \hat{\boldsymbol{W}}^{1 / 2} \boldsymbol{z}^{*}$, nota-se que a matriz $\boldsymbol{H}^{g}$ exerce o papel de matriz de projeção para a variável $\hat{\boldsymbol{W}}^{1 / 2} \boldsymbol{z}^{*}$. Dessa forma, os elementos $h_{i(j j)}^{g}$ da diagonal principal de $\boldsymbol{H}^{g}$ podem ser utilizados para detectar a presença de pontos de alavanca, através do gráfico dos valores $h_{i(j j)}^{g}$ contra os índices associados às observações. Para detectar se a unidade experimental exerce o papel de ponto de alavanca, Venezuela [2003] sugere a utilização dos valores

$$
h_{i .}^{g}=\frac{1}{n_{i}} \sum_{j=1}^{n_{i}} h_{i(j j)}^{g} .
$$

A distância de Cook é utilizada para detectar pontos influentes. Para calcular essa medida, é necessária a obtenção de $\hat{\boldsymbol{\beta}}_{G(i j)}$, que representa a estimativa de $\boldsymbol{\beta}$ obtida com a exclusão da observação $y_{i j}$. Nos modelos marginais de equações de estimação generalizadas, a distância de Cook é definida como

$$
C D_{i j}^{G}=\frac{\left(\hat{\boldsymbol{\beta}}_{G}-\hat{\boldsymbol{\beta}}_{G(i j)}\right)^{\top}\left(\boldsymbol{X}^{\top} \hat{\boldsymbol{W}} \boldsymbol{X}\right)\left(\hat{\boldsymbol{\beta}}_{G}-\hat{\boldsymbol{\beta}}_{G(i j)}\right)}{p} .
$$

Observações influentes podem ser detectadas através do gráfico dos valores $C D_{i j}^{G}$ contra os índices das observações. Outras propostas para a deteç̧ão de observações influentes podem ser encontradas em Preisser e Qaqish [1996] e Pardo e Alonso [2012], por exemplo. Além disso, uma proposta sobre influência local pode ser encontrada em Venezuela et al. [2011]. 


\section{Capítulo 4}

\section{Simulações}

Os estudos de simulação realizados têm como objetivo a avaliação das técnicas de diagnóstico propostas nos Capítulos 2 e 3. Em todos os estudos, foram gerados dados a partir de diferentes modelos condicionais.

Na prática, quando se tem uma amostra com observações correlacionadas, é bastante comum o ajuste de modelos lineares mistos com intercepto aleatório. Diante disso, esse modelo foi ajustado em todos os estudos de simulação, com o objetivo de avaliar se as técnicas de diagnóstico associadas a ele são capazes de detectar que o ajuste desse modelo não é adequado quando os dados são gerados a partir de alguma distribuição que não satisfaz alguma suposição do modelo.

Nos conjuntos de dados gerados, são consideradas amostras com 100 unidades experimentais, sendo cada uma medida $m$ vezes, ou seja, $i=1, \ldots, 100$, e $j=1, \ldots, m$, com $m=4$ (Seções 4.1 e 4.2) ou $m=8$ (Seção 4.3). Além disso, são utilizadas covariáveis $x_{i j}$ geradas a partir de uma distribuição $U[0,8]$. O vetor de parâmetros associados aos efeitos fixos do modelo é $\boldsymbol{\beta}=\left(\beta_{1}, \beta_{2}\right)^{\top}$, em que $\beta_{1}$ é o intercepto e $\beta_{2}$ representa o efeito da covariável $x_{i j}$. Valores conhecidos dos parâmetros são utilizados na simulação.

Primeiramente, a partir da distribuição determinada para os efeitos aleatórios, são geradas as variáveis $u_{i}$ de maneira independente. Em seguida, é definido o vetor de médias condicionais $\boldsymbol{\mu}=\left(\mu_{11}, \ldots, \mu_{100, m}\right)^{\top}$, em que $\mu_{i j}=g^{-1}\left(\eta_{i j}\right)$, para os elementos $\eta_{i j}$ de $\boldsymbol{\eta}=\boldsymbol{X} \boldsymbol{\beta}+\boldsymbol{Z} \boldsymbol{u}$. Esse vetor é utilizado para a geração dos valores da resposta $\boldsymbol{y}$. Em ambas as etapas da geração de variáveis aleatórias, são utilizados valores pré-determinados para os parâmetros de dispersão, quando necessário.

\subsection{Modelos com intercepto aleatório}

\section{Estudo 1}

No primeiro estudo, as observações $y_{i j}$ foram geradas a partir do modelo linear misto dado em (2.2), considerando $\beta_{1}=1$ e $\beta_{2}=2, u_{i} \sim N(0,1)$ independentes, $\varepsilon_{i j} \sim N(0,1)$ independentes e $x_{i j} \sim U[0,8], i=1, \ldots, 100, j=1, \ldots, 4$, com $u_{i}$ e $\varepsilon_{i j}$ independentes.

Primeiramente, foi ajustado um modelo linear misto com intercepto aleatório. As estimativas dos parâmetros do modelo ajustado estão apresentadas na Tabela 4.1. Pode-se notar que as estimativas obtidas estão bastante próximas de seus verdadeiros valores.

A Figura 4.1 apresenta os gráficos dos resíduos marginais e condicionais padronizados versus 


\begin{tabular}{crr}
\hline Parâmetro & Estimativa & Erro padrão \\
\hline$\beta_{1}$ & 1,147 & 0,154 \\
$\beta_{2}$ & 1,980 & 0,026 \\
$\sigma_{u}^{2}$ & 1,074 & \\
$\sigma^{2}$ & 1,115 & \\
\hline
\end{tabular}

Tabela 4.1: Estimativas (erros padrões) dos parâmetros do modelo linear misto com intercepto aleatório - estudo 1.

os índices das unidades experimentais. Os gráficos não indicam nenhuma observação discrepante. Os gráficos dos resíduos marginais e condicionais padronizados versus os valores ajustados confirmam a linearidade dos efeitos fixos e a homocedasticidade dos erros condicionais (Figura 4.2). O gráfico para a estrutura de covariância indica que a estrutura de covariância adotada no modelo está adequada (Figura 4.3).

Os gráficos Q-Q dos resíduos marginais padronizados, condicionais padronizados (Figura 4.4) e minimamente confundidos (Figura 4.5) indicam que o modelo está bem ajustado, não havendo indícios de que os erros marginais e condicionais tenham distribuição diferente da normal. Além disso, os gráficos Q-Q dos resíduos $t_{i j}^{*}$ e dos resíduos quantílicos também apontam para a validade do modelo proposto (Figuras 4.6 e 4.7).

Na Figura 4.8, o gráfico das distâncias de Mahalanobis $\left(\hat{\zeta}_{i}\right)$ versus os índices das unidades experimentais não detecta nenhuma unidade experimental discrepante e o gráfico Q-Q dessas distâncias indica que a distribuição dos efeitos aleatórios é normal.

As Figuras 4.9 e 4.10 apresentam as medidas de alavanca baseadas em $\boldsymbol{H}^{m}$ e $\boldsymbol{H}^{c}$, respectivamente. É possível observar que as duas matrizes de projeção sugeridas apresentam resultados parecidos. O gráfico das distâncias de Cook (Figura 4.11) destaca a observação 4 da unidade experimental número 3 como possível observação influente. Ajustando o modelo sem esta observação, obtemos $\hat{\beta}_{1}=1,177$ e $\hat{\beta}_{2}=1,974$, com $\hat{\sigma}^{2}=1,104$ e $\hat{\sigma}_{u}^{2}=1,042$, o que não representa grande diferença com relação aos valores apresentados na Tabela 4.1.

As variáveis aleatórias geradas resultam na distribuição marginal $\boldsymbol{y}_{i} \sim N\left(\boldsymbol{X}_{i} \boldsymbol{\beta}, \boldsymbol{V}_{i}\right)$, com

$$
\boldsymbol{V}_{i}=\left[\begin{array}{llll}
2 & 1 & 1 & 1 \\
1 & 2 & 1 & 1 \\
1 & 1 & 2 & 1 \\
1 & 1 & 1 & 2
\end{array}\right]
$$

para $i=1, \ldots, 100$. Assim, a matriz de correlações para cada unidade experimental é dada por

$$
\boldsymbol{R}=\left[\begin{array}{cccc}
1 & 0,5 & 0,5 & 0,5 \\
0,5 & 1 & 0,5 & 0,5 \\
0,5 & 0,5 & 1 & 0,5 \\
0,5 & 0,5 & 0,5 & 1
\end{array}\right]
$$

O próximo passo foi ajustar um modelo marginal com estrutura de correlação uniforme dada 
em (3.1). O resumo do ajuste desse modelo é apresentado na Tabela 4.2. Como esperado, as estimativas de $\hat{\beta}_{1}$ e $\hat{\beta}_{2}$ são praticamente as mesmas obtidas no ajuste do modelo linear misto. Os valores estimados dos erros padrões naive e robusto dos estimadores desses parâmetros do modelo marginal são próximos dos valores obtidos no ajuste do modelo linear misto.

\begin{tabular}{crrr}
\hline Parâmetro & Estimativa & EP naive & EP robusto \\
\hline$\beta_{1}$ & 1,147 & 0,153 & 0,151 \\
$\beta_{2}$ & 1,980 & 0,026 & 0,022 \\
$\phi^{-1}$ & 2,311 & & \\
$\alpha$ & 0,488 & & \\
\hline
\end{tabular}

Tabela 4.2: Estimativas (erros padrões) dos parâmetros do modelo marginal normal com estrutura de correlação uniforme - estudo 1.

O gráfico dos resíduos do modelo marginal normal é semelhante ao gráfico dos resíduos marginais padronizados obtido no modelo linear misto. O gráfico Q-Q desses resíduos também indica a normalidade da variável resposta (Figura 4.12). Os gráficos das medidas de alavanca desse ajuste estão na Figura 4.13. A Figura 4.14 mostra os valores da distância de Cook, destacando a observação de número 4 da unidade experimental 3. Removendo esta observação, obtemos $\hat{\beta}_{1}=1,177, \hat{\beta}_{2}=1,974, \hat{\phi}^{-1}=2,135$ e $\hat{\alpha}=0,477$, diferença pequena com relação aos valores apresentados na Tabela 4.2. Isso indica que não há observações influentes nesse caso.

Para os dados simulados com base em um modelo linear misto com intercepto aleatório, as técnicas de diagnóstico utilizadas indicaram corretamente o bom ajuste dos modelos sob as duas abordagens.
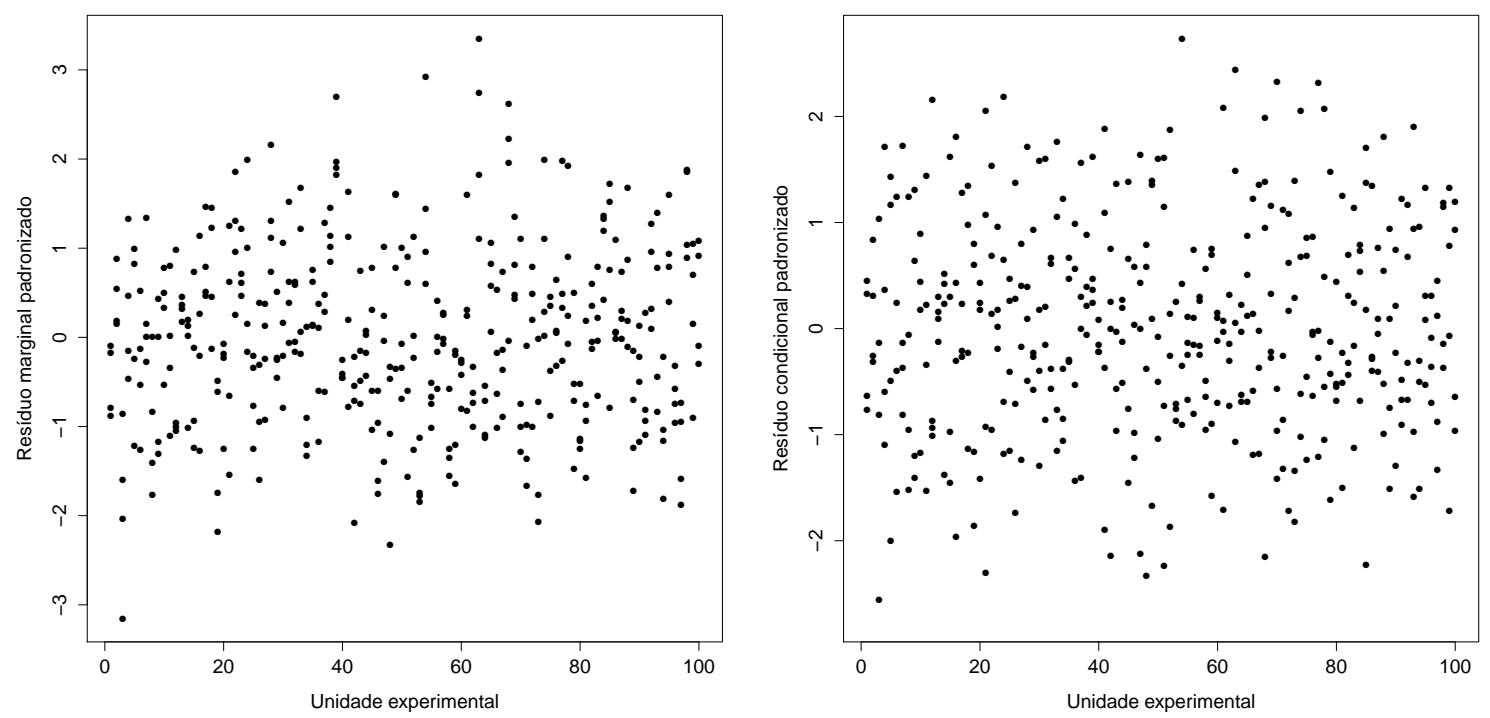

Figura 4.1: Resíduos marginais e condicionais padronizados versus os indices das unidades experimentais do modelo linear misto com intercepto aleatório - estudo 1. 

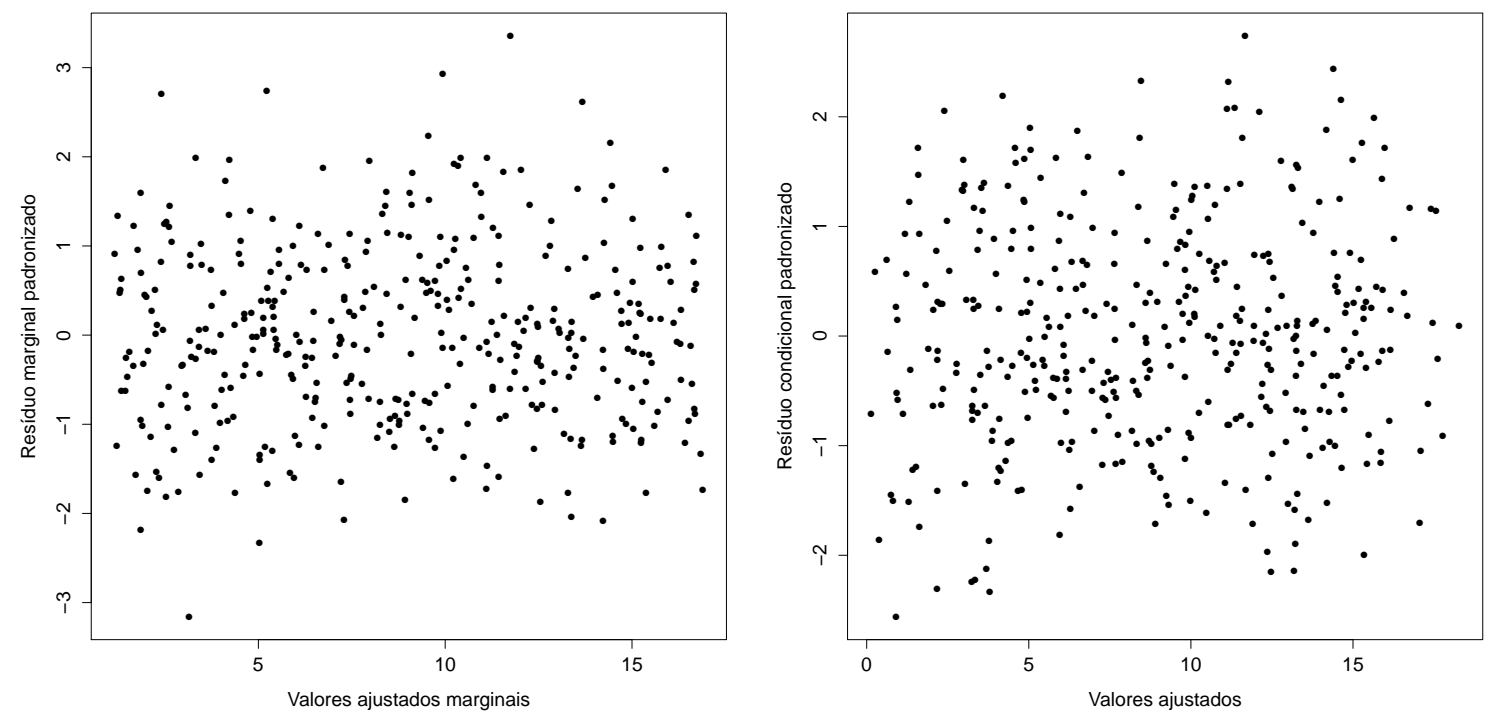

Figura 4.2: Resíduos marginais e condicionais padronizados versus os valores ajustados do modelo linear misto com intercepto aleatório - estudo 1.

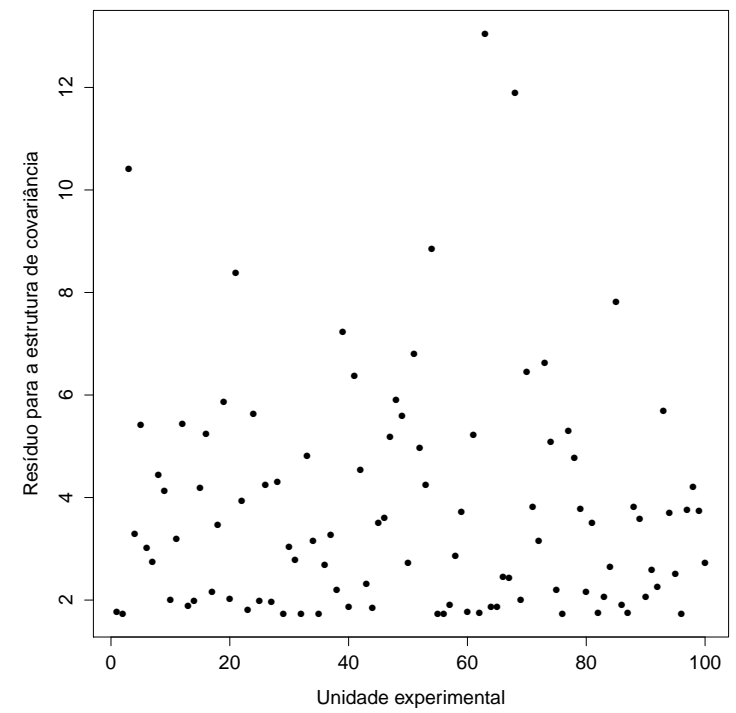

Figura 4.3: Gráfico dos resíduos para a estrutura de covariância versus os índices das unidades experimentais do modelo linear misto com intercepto aleatório - estudo 1. 

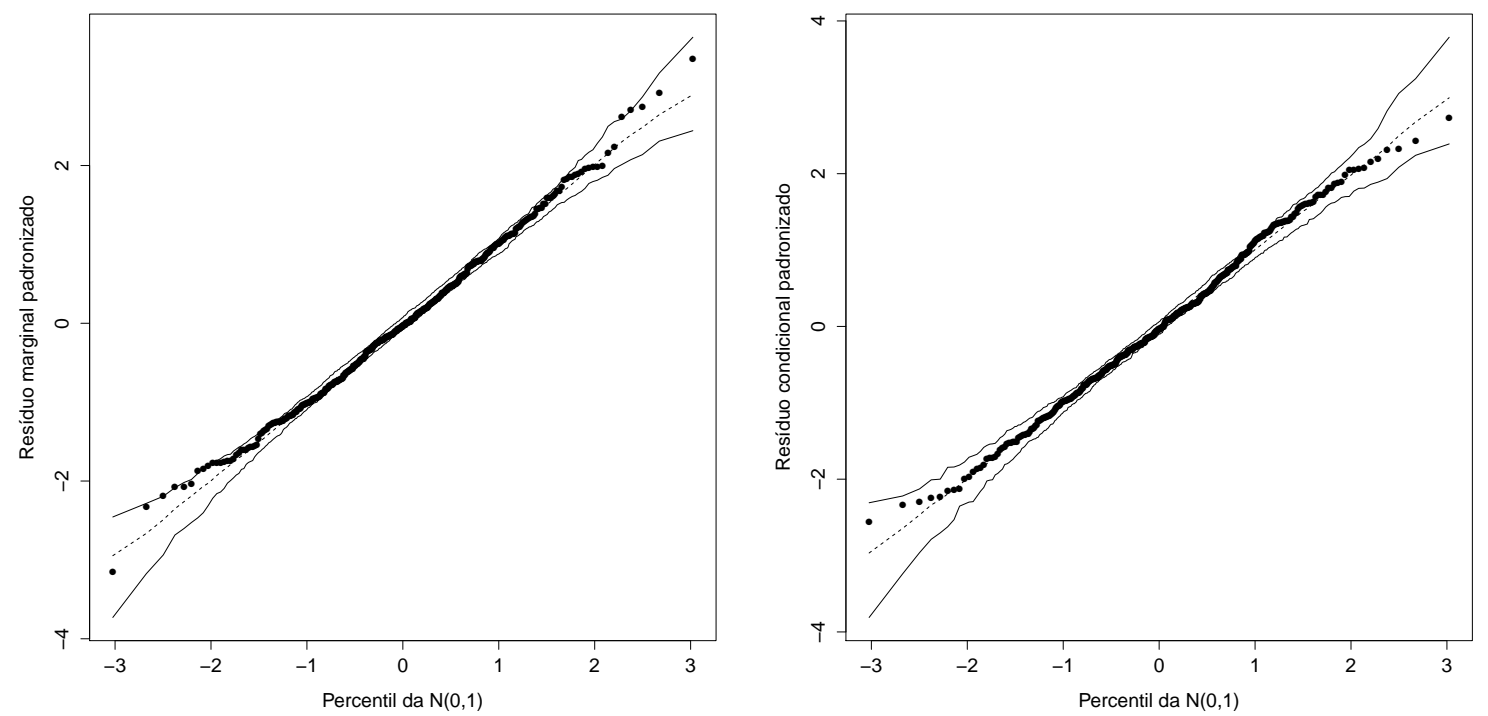

Figura 4.4: Gráficos $Q-Q$ dos resíduos marginais e condicionais padronizados do modelo linear misto com intercepto aleatório - estudo 1.

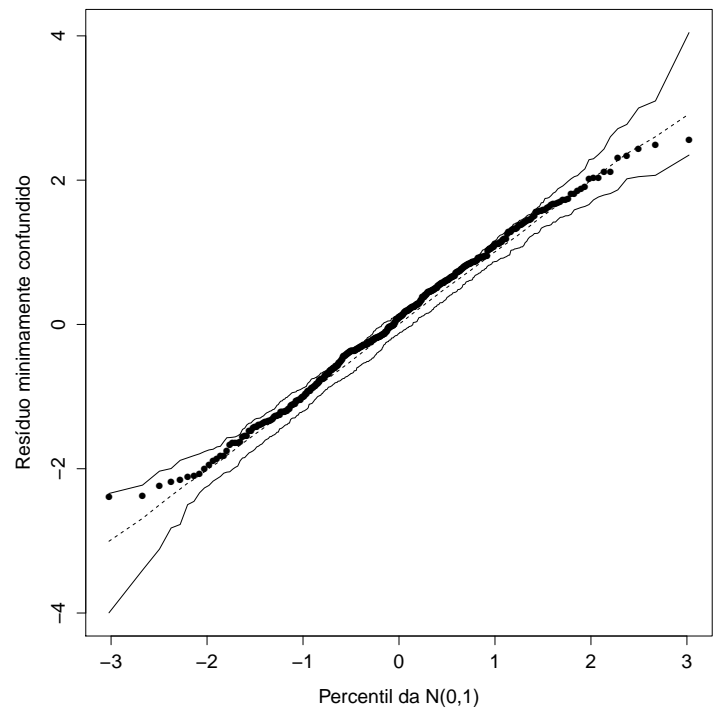

Figura 4.5: Gráfico $Q-Q$ dos resíduos minimamente confundidos do modelo linear misto com intercepto aleatório - estudo 1. 


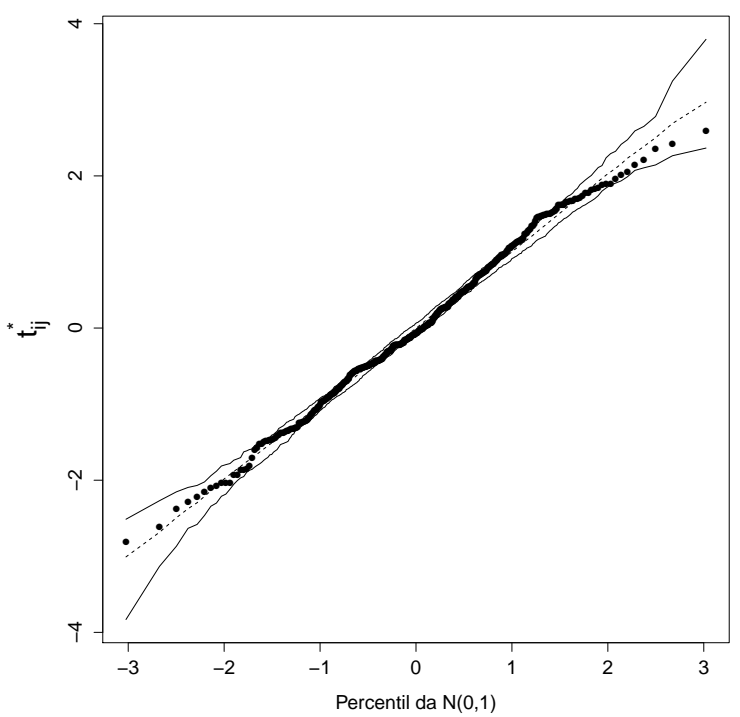

Figura 4.6: Gráfico $Q-Q$ dos resíduos $t_{i j}^{*}$ do modelo linear misto com intercepto aleatório - estudo 1.

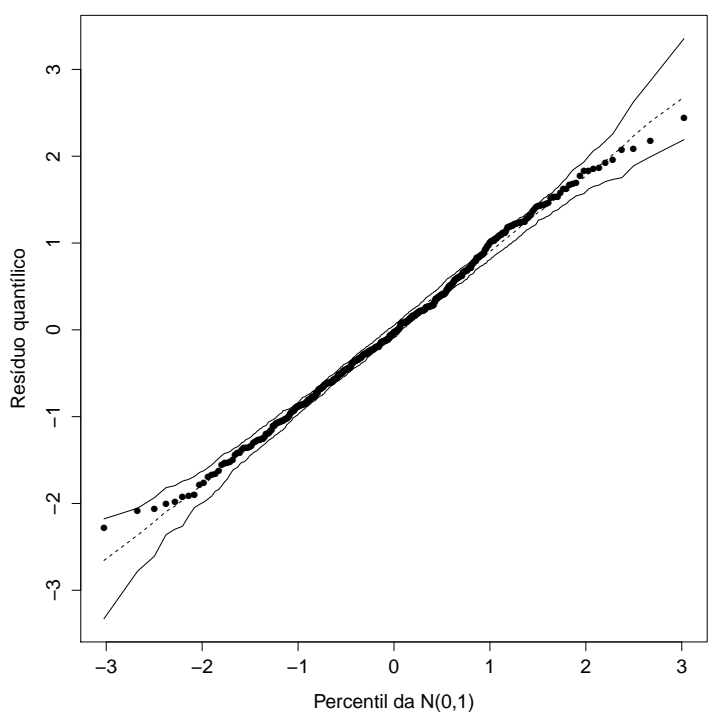

Figura 4.7: Gráfico $Q-Q$ dos resíduos quantílicos do modelo linear misto com intercepto aleatório estudo 1. 

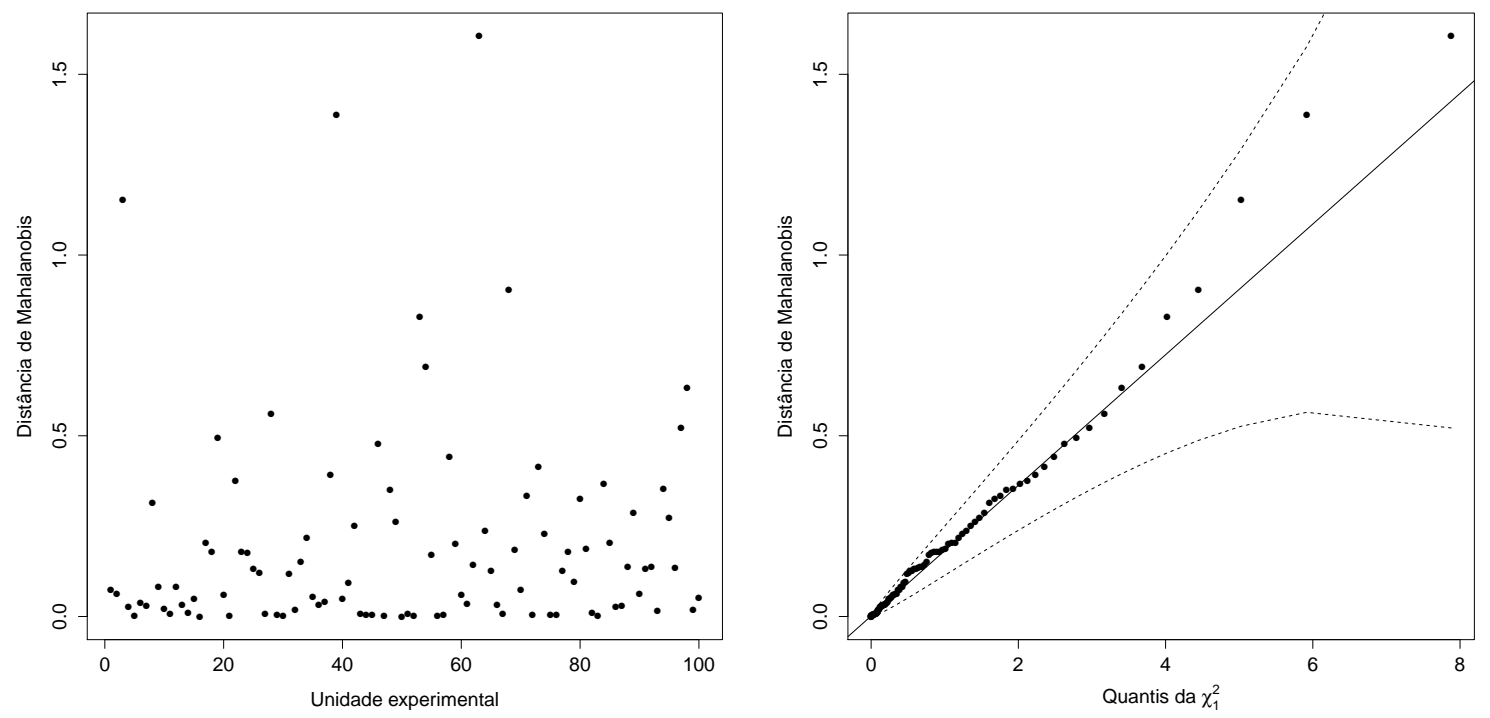

Figura 4.8: Gráficos das distâncias de Mahalanobis do modelo linear misto com intercepto aleatório estudo 1.
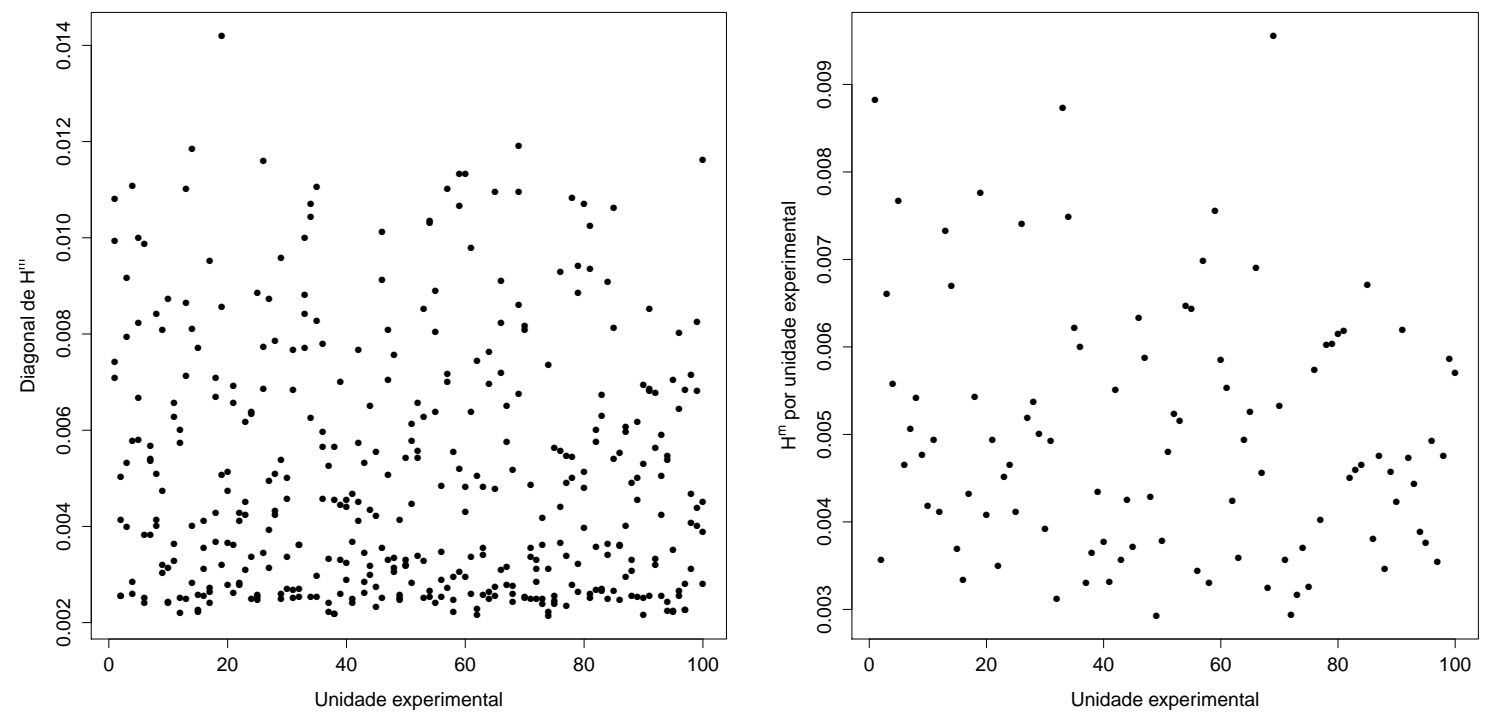

Figura 4.9: Gráficos das medidas de alavanca com base em $\boldsymbol{H}^{m}$ versus os indices das unidades experimentais do modelo linear misto com intercepto aleatório - estudo 1. 

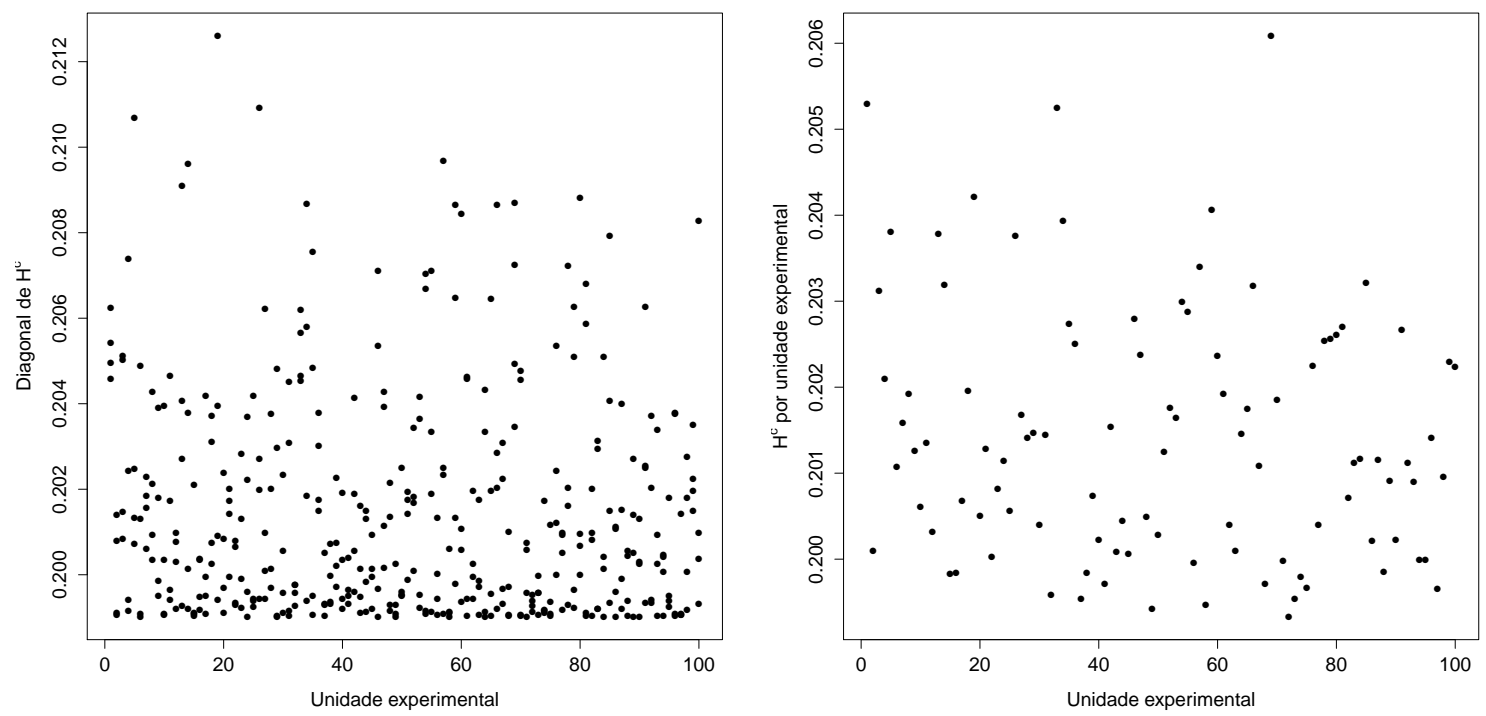

Figura 4.10: Gráficos das medidas de alavanca com base em $\boldsymbol{H}^{c}$ versus os índices das unidades experimentais do modelo linear misto com intercepto aleatório - estudo 1.

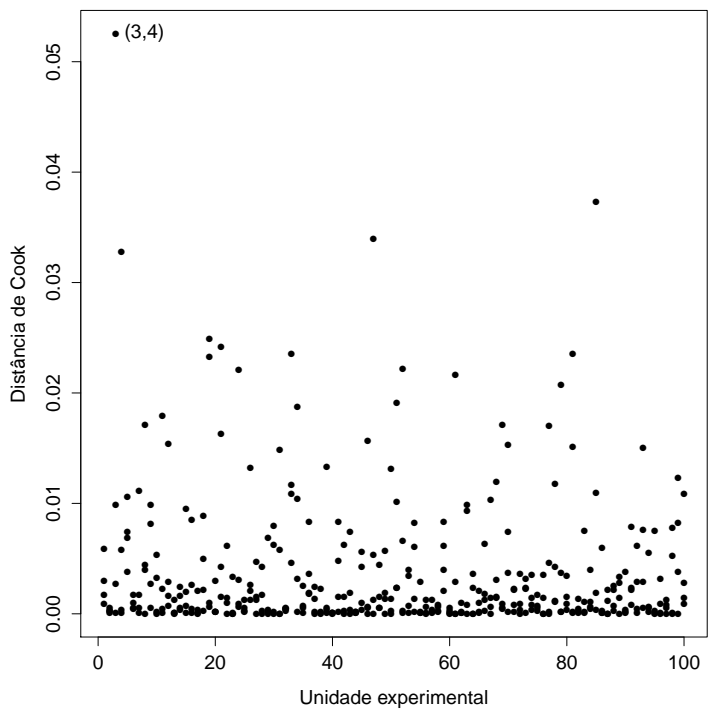

Figura 4.11: Gráfico das distâncias de Cook versus os índices das unidades experimentais do modelo linear misto com intercepto aleatório - estudo 1. 

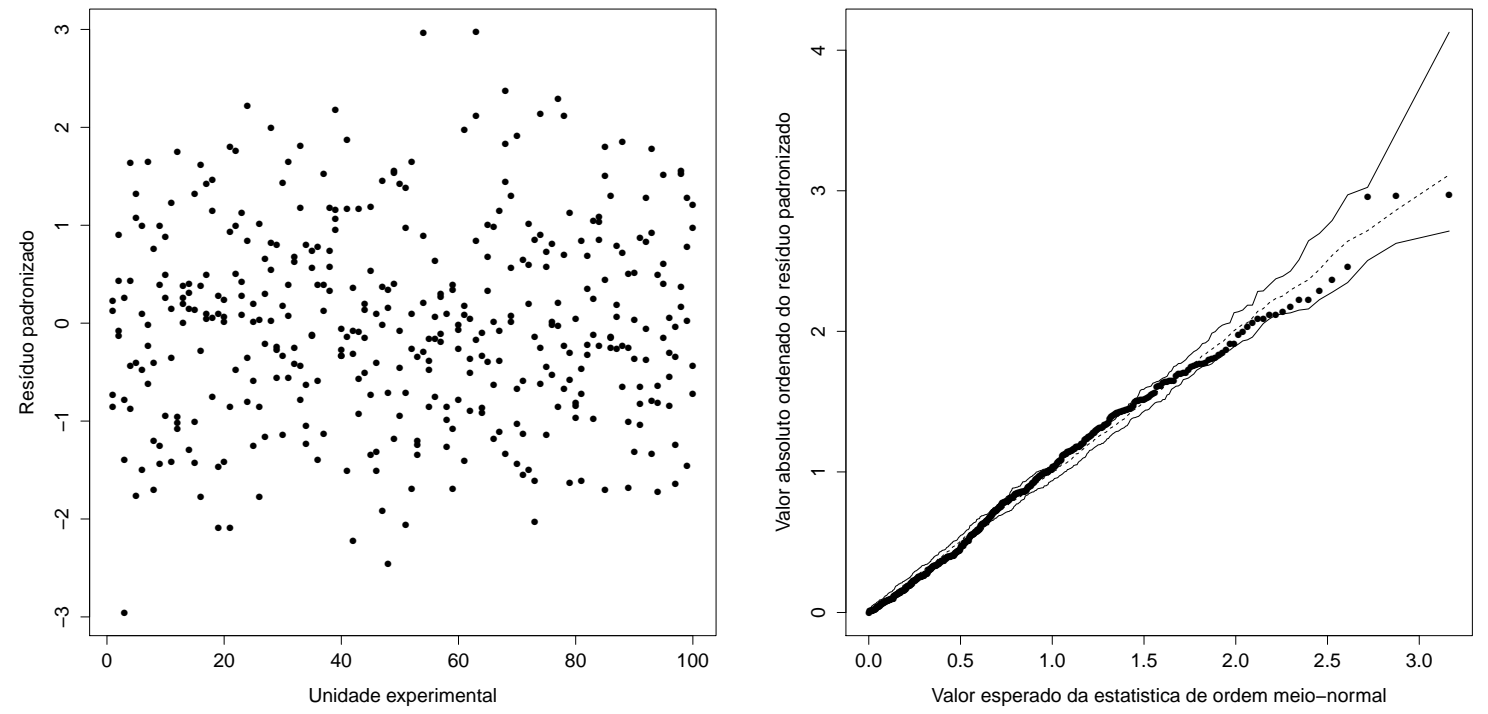

Figura 4.12: Gráficos dos resíduos padronizados do modelo marginal normal com estrutura de correlação uniforme - estudo 1.
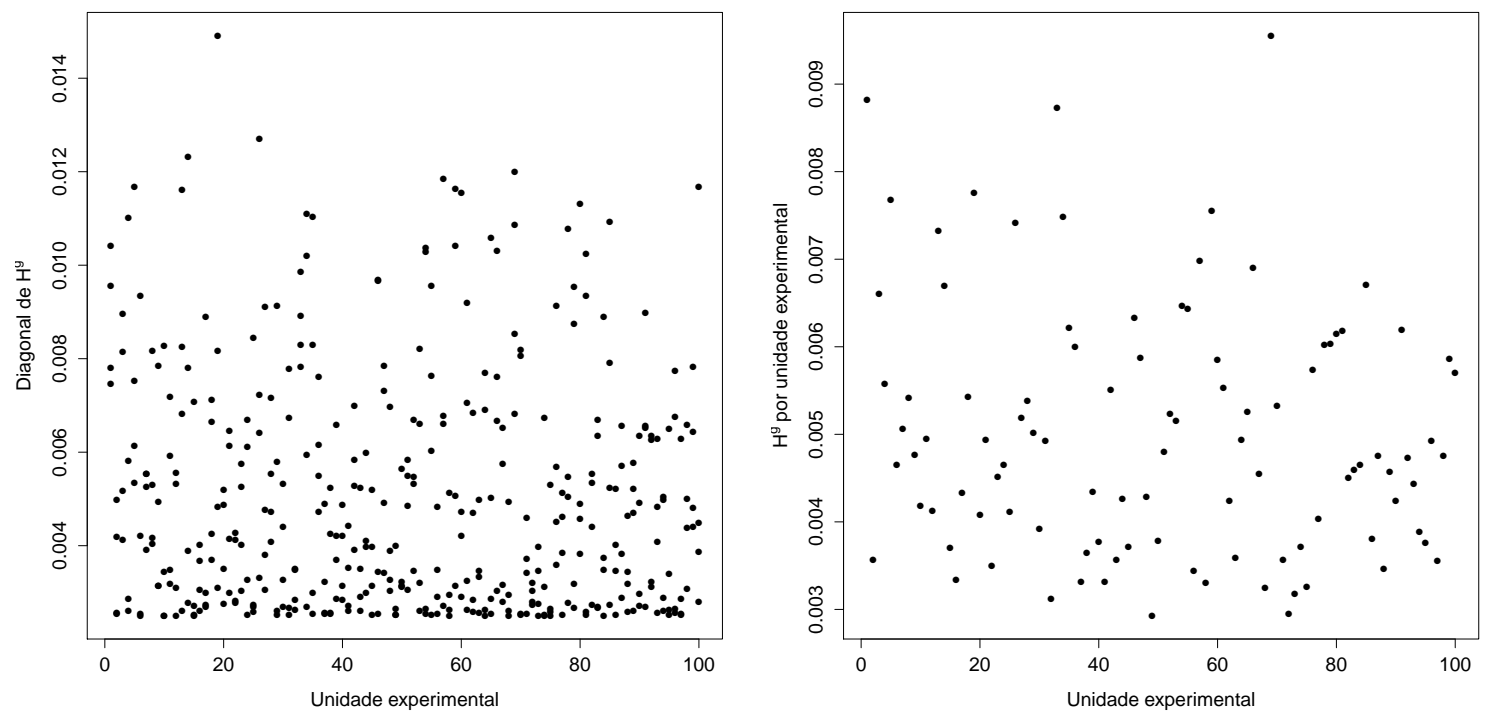

Figura 4.13: Gráficos das medidas de alavanca com base em $\boldsymbol{H}^{g}$ versus os índices das unidades experimentais do modelo marginal normal com estrutura de correlação uniforme - estudo 1. 


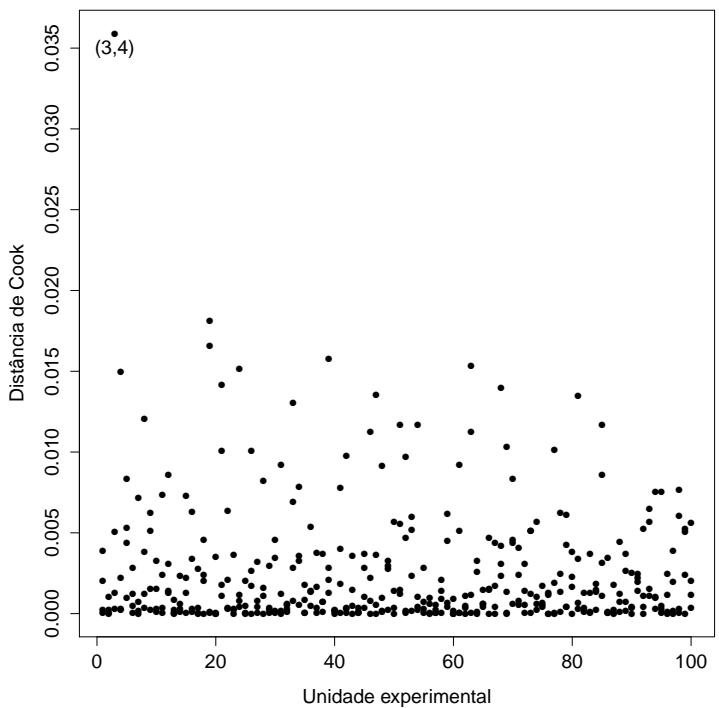

Figura 4.14: Gráfico das distâncias de Cook versus os índices das unidades experimentais do modelo marginal normal com estrutura de correlação uniforme - estudo 1. 


\section{Estudo 2}

No segundo estudo de simulação, as observações foram geradas como no Estudo 1, exceto que a distribuição dos efeitos aleatórios foi alterada, sendo $u_{i} \sim(1 / \sqrt{3}) t_{3}$.

Foram ajustados dois modelos: um modelo linear misto com intercepto aleatório e um modelo marginal normal com matriz de correlações de trabalho uniforme. As Tabelas 4.3 e 4.4 apresentam os resultados dos ajustes dos dois modelos.

Para o modelo linear misto, nenhuma observação é detectada como discrepante pelo gráfico dos resíduos condicionais padronizados, mas o gráfico dos resíduos marginais padronizados indica algumas unidades experimentais discrepantes (Figura 4.15).

\begin{tabular}{crr}
\hline Parâmetro & Estimativa & Erro padrão \\
\hline$\beta_{1}$ & 0,880 & 0,156 \\
$\beta_{2}$ & 2,063 & 0,024 \\
$\sigma_{u}^{2}$ & 1,341 & \\
$\sigma^{2}$ & 0,925 & \\
\hline
\end{tabular}

Tabela 4.3: Estimativas (erros padrões) dos parâmetros do modelo linear misto com intercepto aleatório - estudo 2.

A Figura 4.16 indica a linearidade dos efeitos fixos e a homocedasticidade dos erros condicionais. Na Figura 4.17, é possível notar que algumas unidades experimentais apresentaram valores maiores dos resíduos para a estrutura de covariância do que aqueles obtidos na simulação com intercepto aleatório normal (Figura 4.3), mostrando que a estrutura de covariância adotada no modelo pode não estar adequada da mesma maneira para todas as unidades experimentais.

O gráfico Q-Q dos resíduos marginais padronizados não indica um bom ajuste nesse caso, enquanto o gráfico Q-Q para os resíduos condicionais padronizados aponta para a normalidade dos erros condicionais (Figura 4.18). Pode-se perceber que a alteração na distribuição dos efeitos aleatórios afetou a distribuição dos erros marginais, o que não ocorreu com a distribuição dos erros condicionais. Nas Figuras 4.19 a 4.21, os gráficos Q-Q dos resíduos minimamente confundidos, dos resíduos $t_{i j}^{*}$ e dos resíduos quantílicos também indicam a normalidade dos erros condicionais.

O gráfico das distâncias de Mahalanobis destaca algumas unidades experimentais como discrepantes, e o gráfico Q-Q dessas distâncias consegue detectar que a distribuição normal não é a mais adequada para os efeitos aleatórios (Figura 4.22).

Como foi utilizada a mesma matriz $\boldsymbol{X}$ do estudo 1, os gráficos das medidas de alavanca do estudo 2 (Figuras 4.23 e 4.24) são muito parecidos com aqueles do estudo 1. A observação 3 da unidade experimental 19 teve o valor mais alto da medida de influência (Figura 4.25). Eliminando esta observação, não há grande alteração nas estimativas dos parâmetros fixos, com $\hat{\beta}_{1}=0,879, \hat{\beta}_{2}=2,063, \hat{\sigma}^{2}=0,928$ e $\hat{\sigma}_{u}^{2}=1,340$.

Para o modelo marginal normal com estrutura uniforme (Tabela 4.4), o gráfico dos resíduos padronizados mostra a presença de poucas observações discrepantes, enquanto o gráfico Q-Q indica um bom ajuste do modelo (Figura 4.26).

As Figuras 4.27 e 4.28 apresentam as medidas de alavanca e de influência das observações, respectivamente. A observação 3 da unidade experimental 19 foi aquela com o maior valor da 


\begin{tabular}{crrr}
\hline Parâmetro & Estimativa & EP naive & EP robusto \\
\hline$\beta_{1}$ & 0,880 & 0,155 & 0,152 \\
$\beta_{2}$ & 2,063 & 0,024 & 0,025 \\
$\phi^{-1}$ & 2,259 & & \\
$\alpha$ & 0,589 & & \\
\hline
\end{tabular}

Tabela 4.4: Estimativas (erros padrões) dos parâmetros do modelo marginal normal com estrutura de correlação uniforme - estudo 2.

distância de Cook. Porém, não deve ser considerada como observação influente, pois o ajuste sem elas resulta em $\hat{\beta}_{1}=0,880, \hat{\beta}_{2}=2,063, \hat{\phi}^{-1}=2,264$ e $\hat{\alpha}=0,560$, o que não representa grande alteração nas estimativas dos parâmetros.
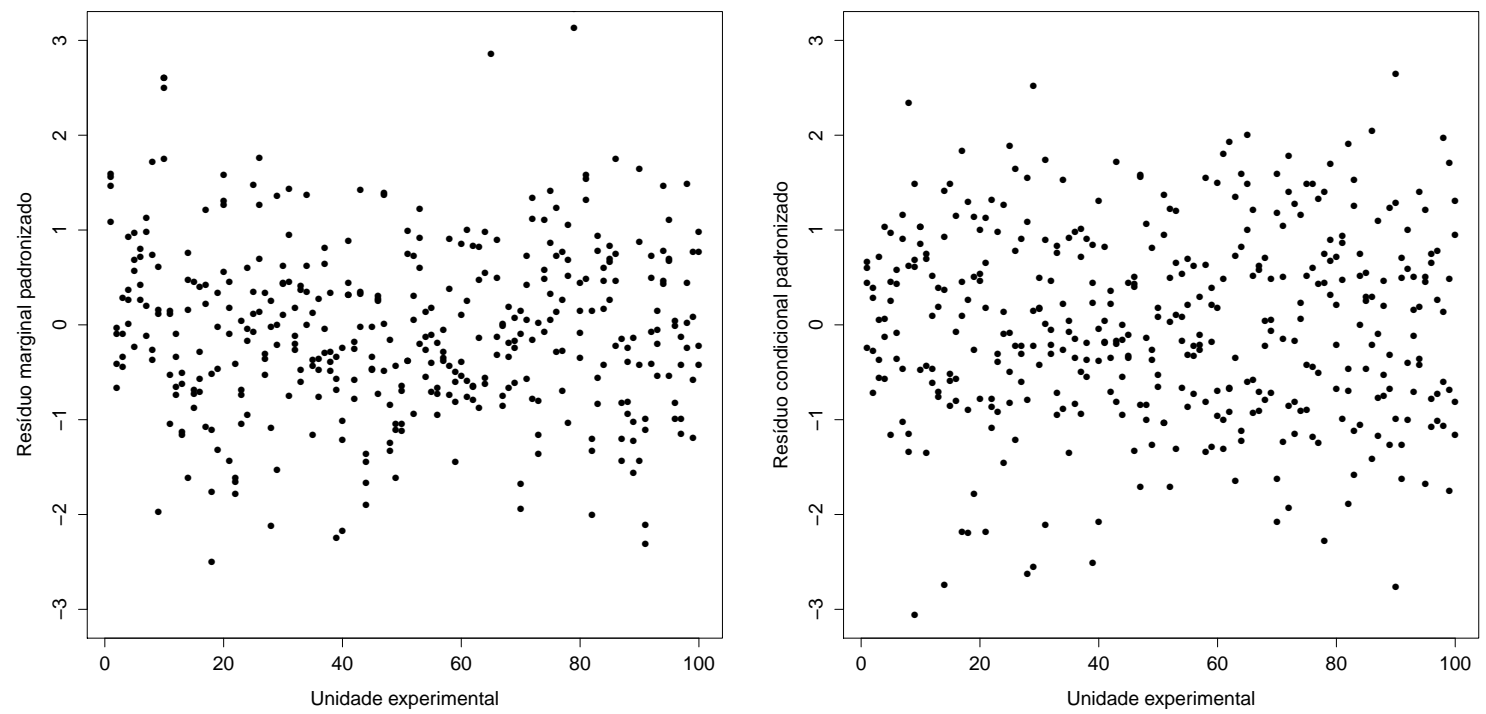

Figura 4.15: Resíduos marginais e condicionais padronizados versus os índices das unidades experimentais do modelo linear misto com intercepto aleatório - estudo 2. 

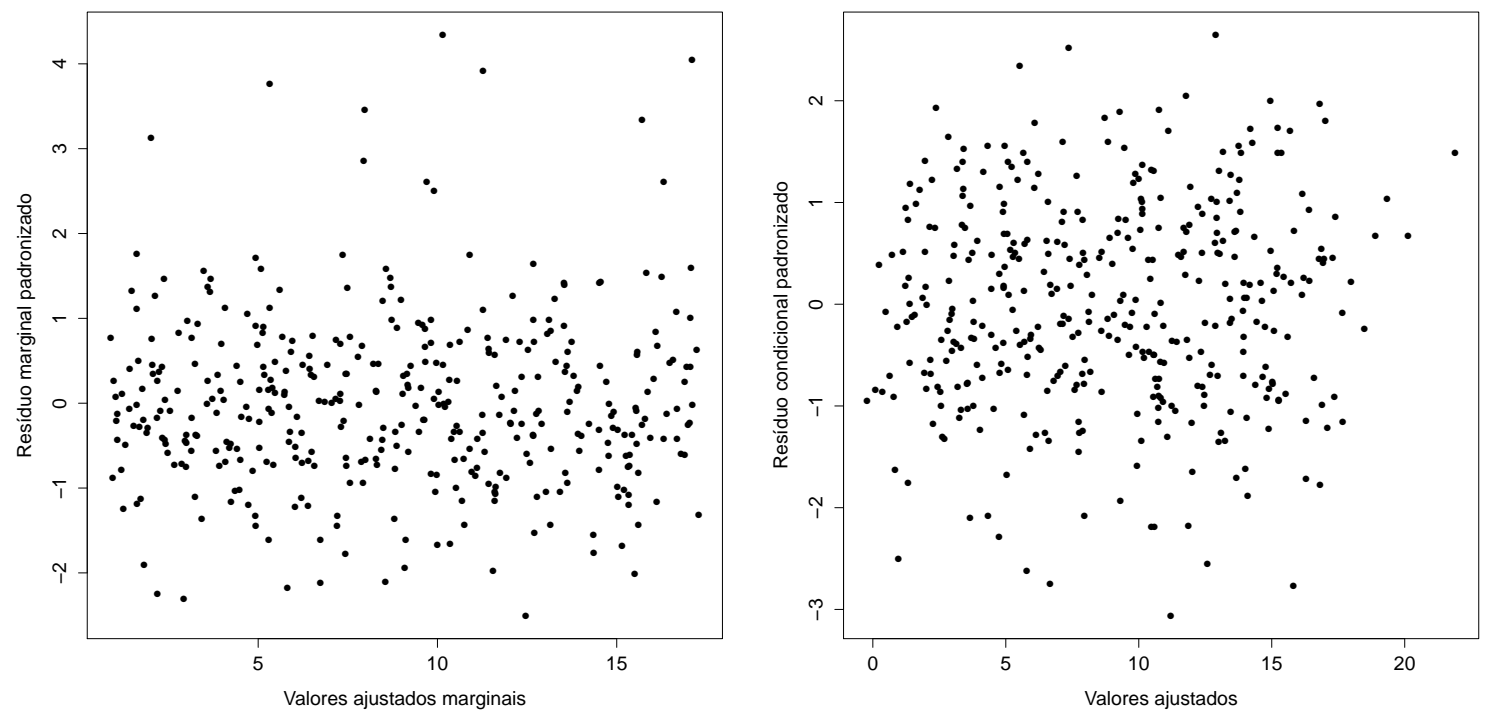

Figura 4.16: Resíduos marginais e condicionais padronizados versus os valores ajustados do modelo linear misto com intercepto aleatório - estudo 2.

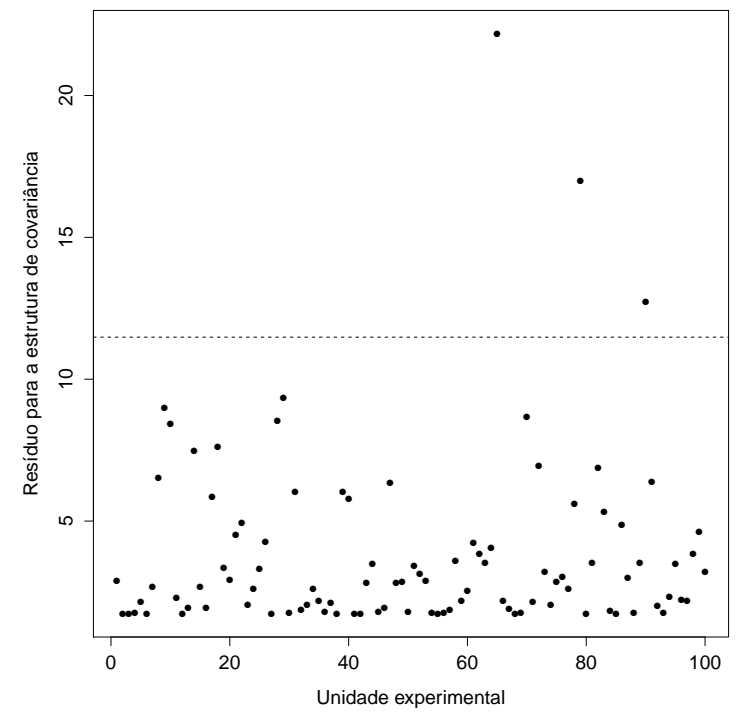

Figura 4.17: Gráfico dos resíduos para a estrutura de covariância versus os índices das unidades experimentais do modelo linear misto com intercepto aleatório - estudo 2. 

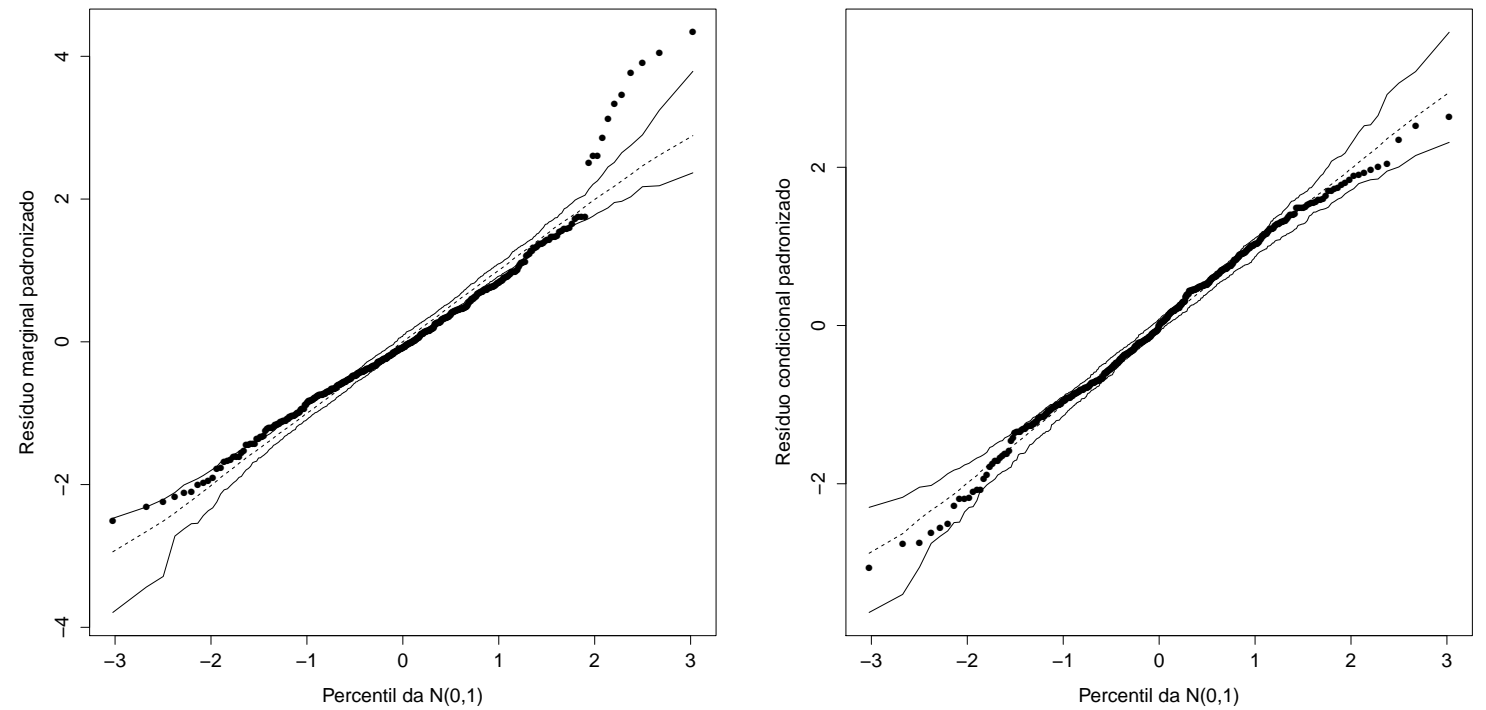

Figura 4.18: Gráficos $Q-Q$ dos resíduos marginais e condicionais padronizados do modelo linear misto com intercepto aleatório - estudo 2.

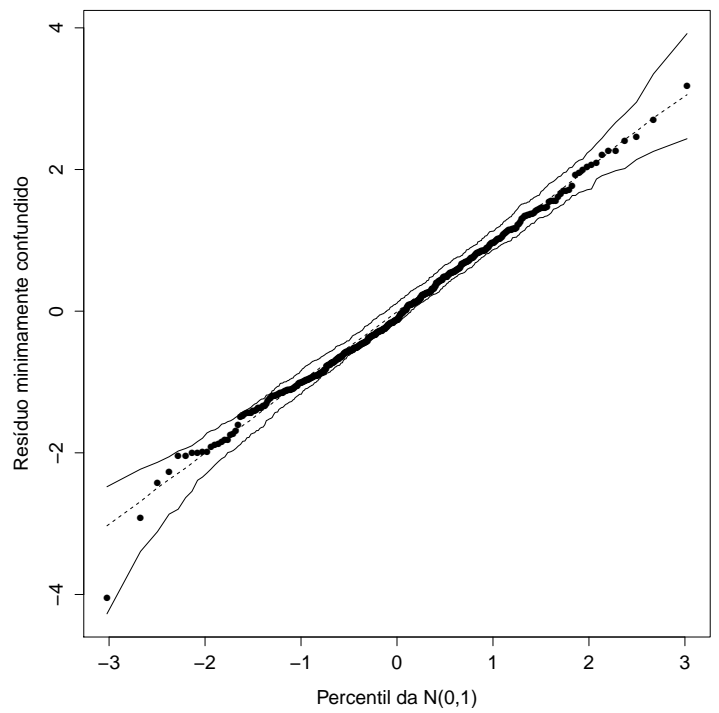

Figura 4.19: Gráfico $Q-Q$ dos resíduos minimamente confundidos do modelo linear misto com intercepto aleatório - estudo 2. 


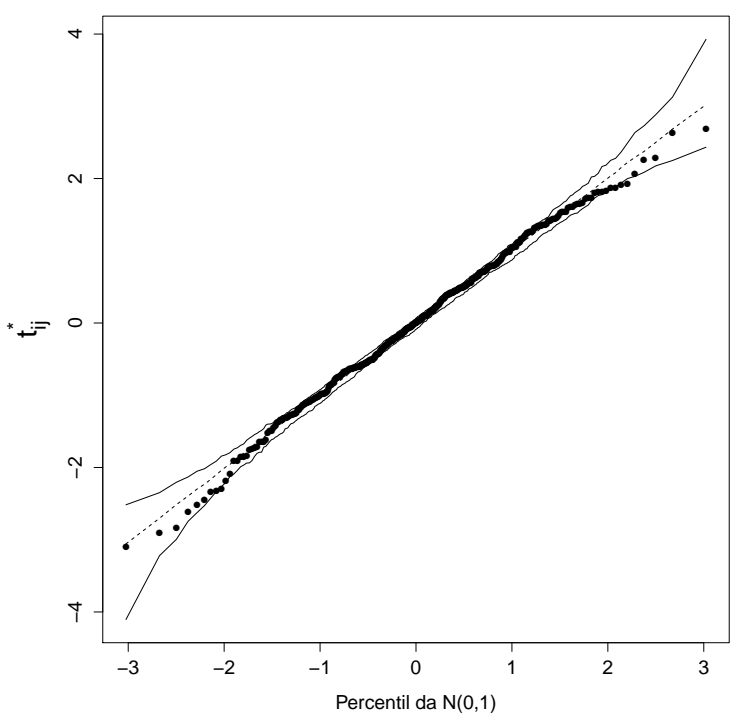

Figura 4.20: Gráfico $Q-Q$ dos resíduos $t_{i j}^{*}$ do modelo linear misto com intercepto aleatório - estudo 2.

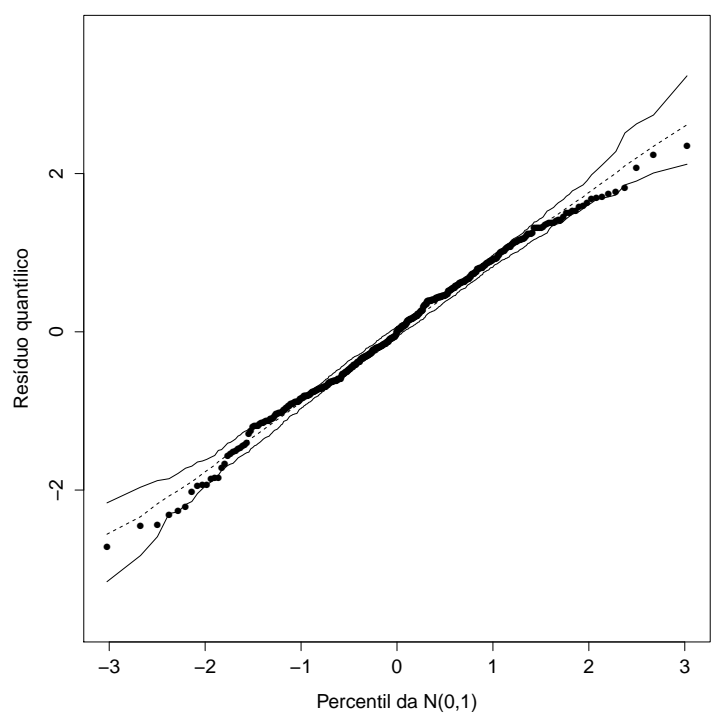

Figura 4.21: Gráfico $Q-Q$ dos resíduos quantílicos do modelo linear misto com intercepto aleatório estudo 2. 

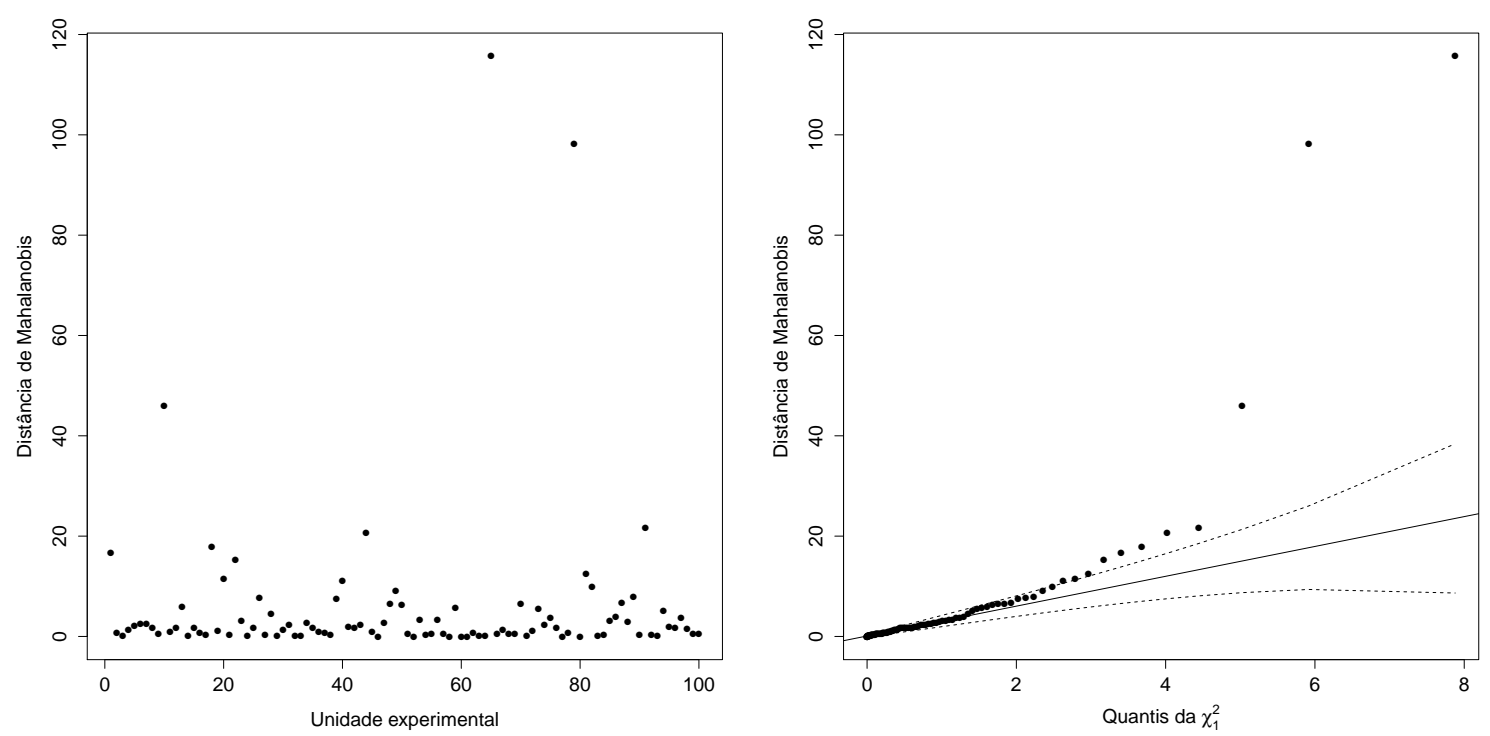

Figura 4.22: Gráficos das distâncias de Mahalanobis do modelo linear misto com intercepto aleatório estudo 2.
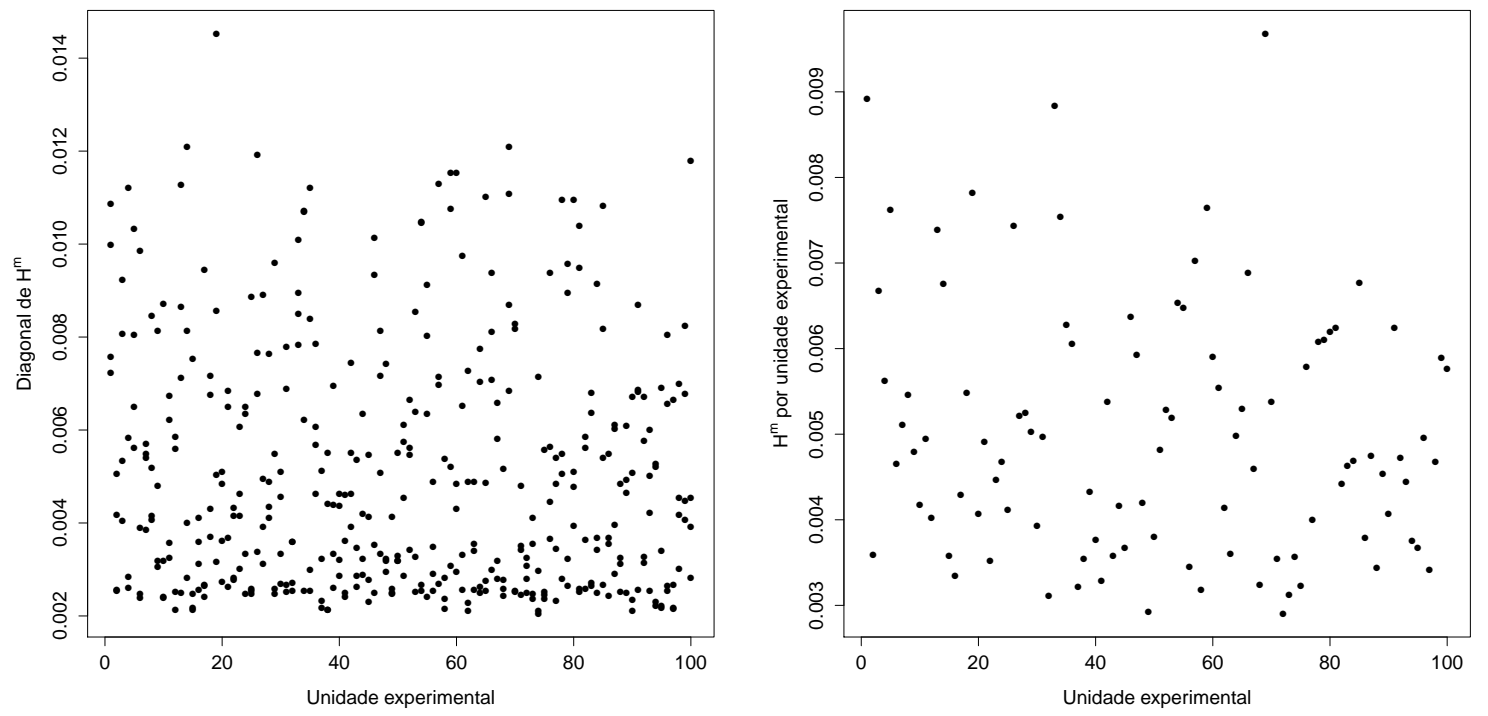

Figura 4.23: Gráficos das medidas de alavanca com base em $\boldsymbol{H}^{m}$ versus os índices das unidades experimentais do modelo linear misto com intercepto aleatório - estudo 2. 

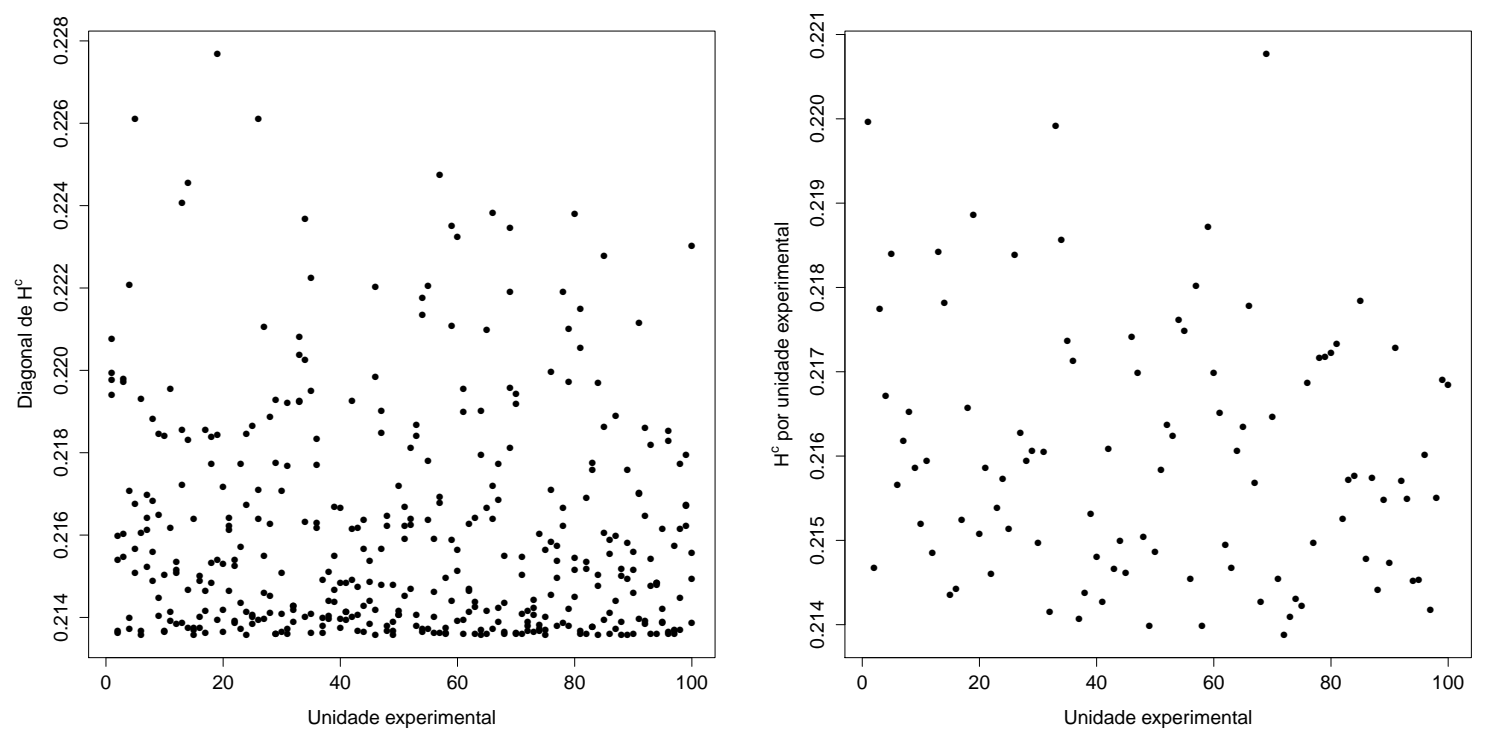

Figura 4.24: Gráficos das medidas de alavanca com base em $\boldsymbol{H}^{c}$ versus os índices das unidades experimentais do modelo linear misto com intercepto aleatório - estudo 2.

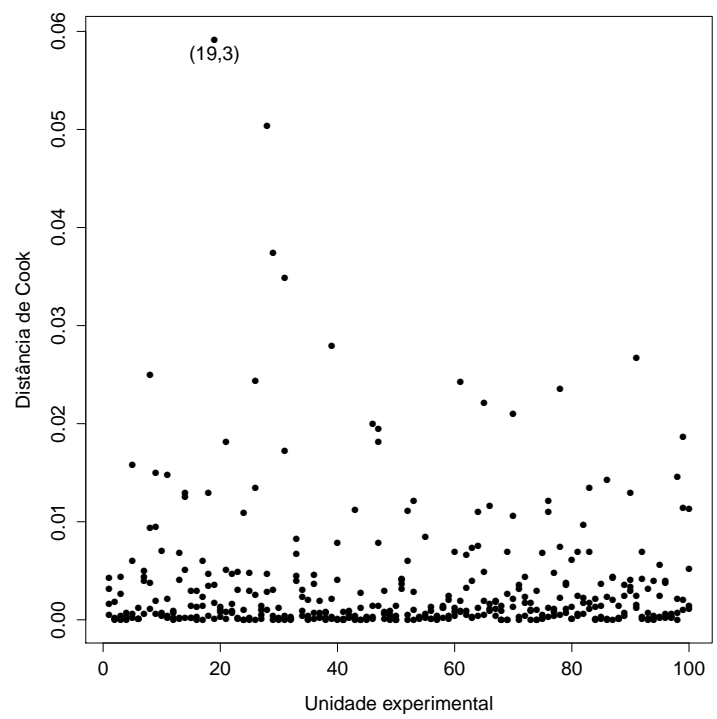

Figura 4.25: Gráfico das distâncias de Cook versus os índices das unidades experimentais do modelo linear misto com intercepto aleatório - estudo 2. 

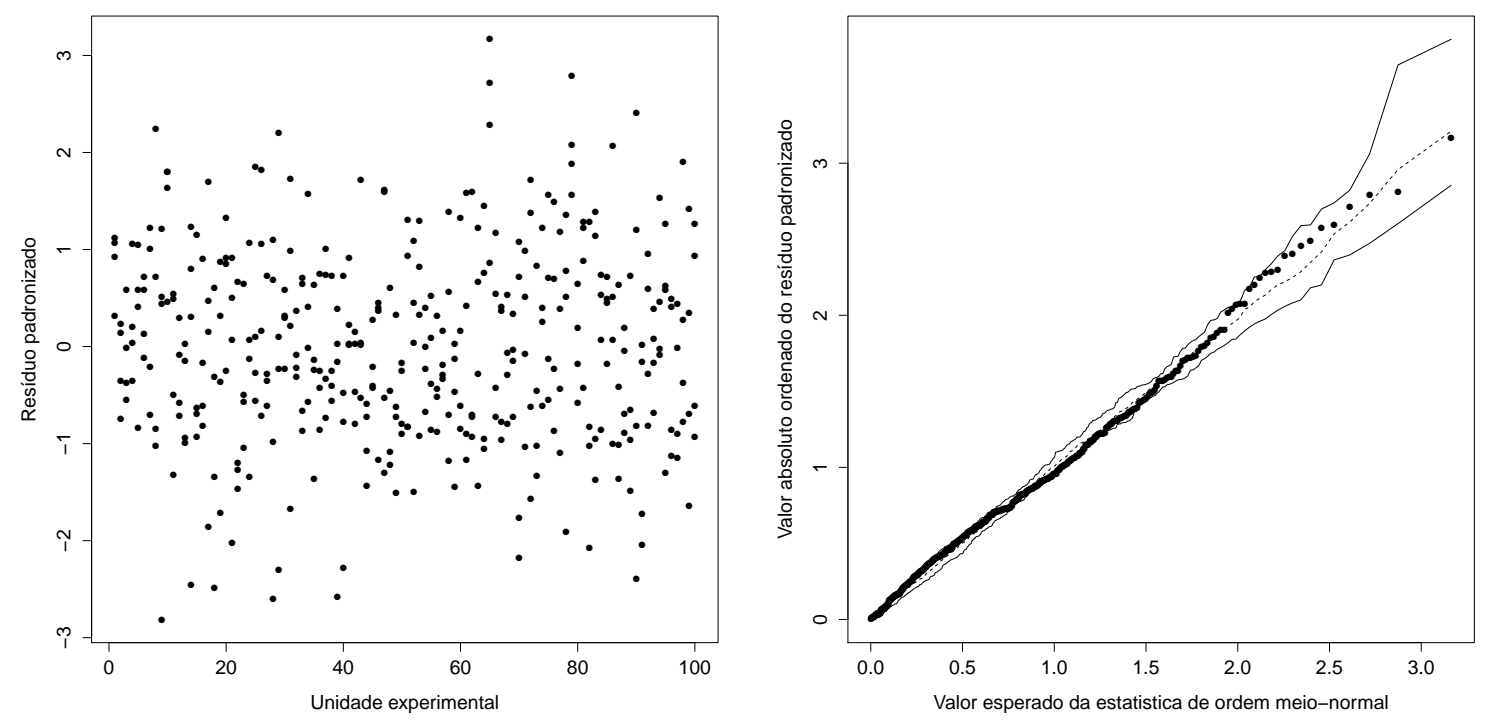

Figura 4.26: Gráficos dos resíduos padronizados do modelo marginal normal com estrutura de correlação uniforme - estudo 2.
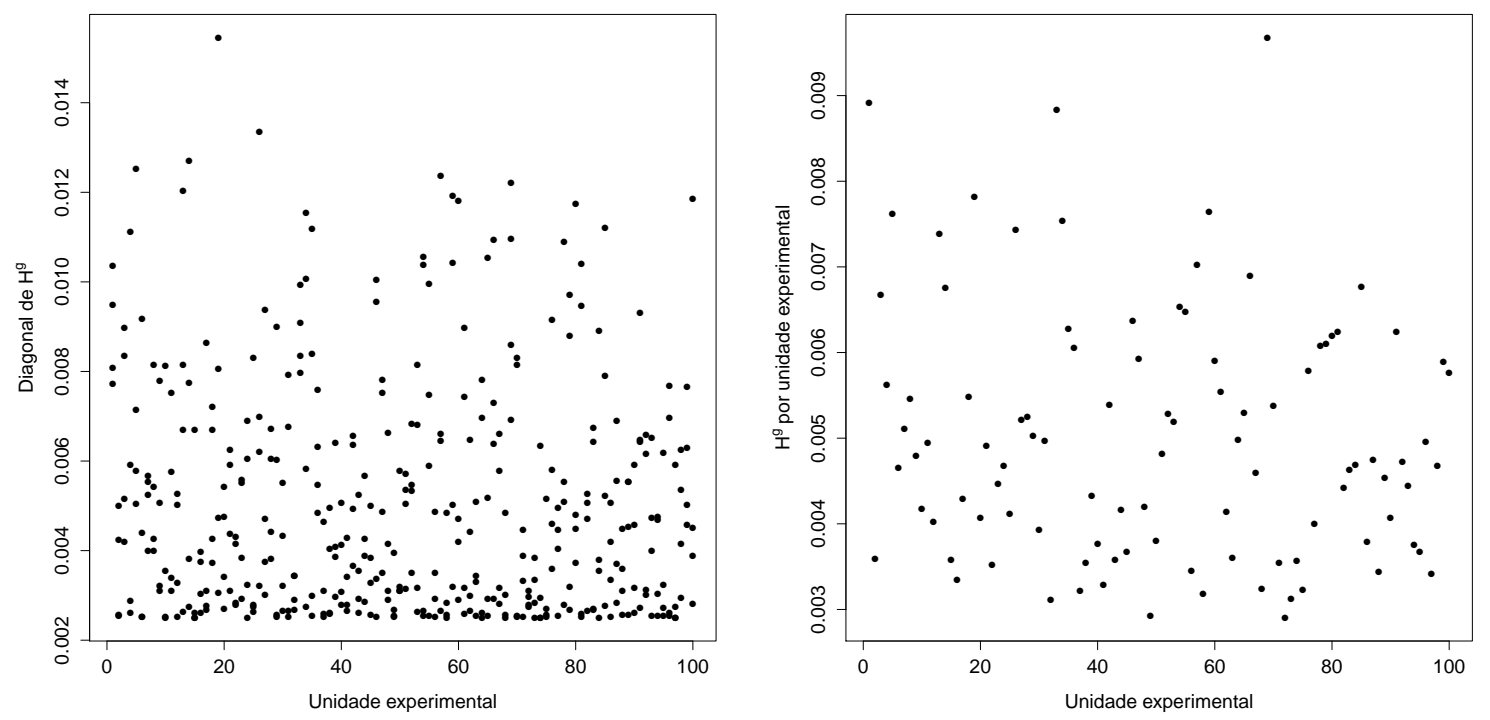

Figura 4.27: Gráficos das medidas de alavanca com base em $\boldsymbol{H}^{g}$ versus os índices das unidades experimentais do modelo marginal normal com estrutura de correlação uniforme - estudo 2. 


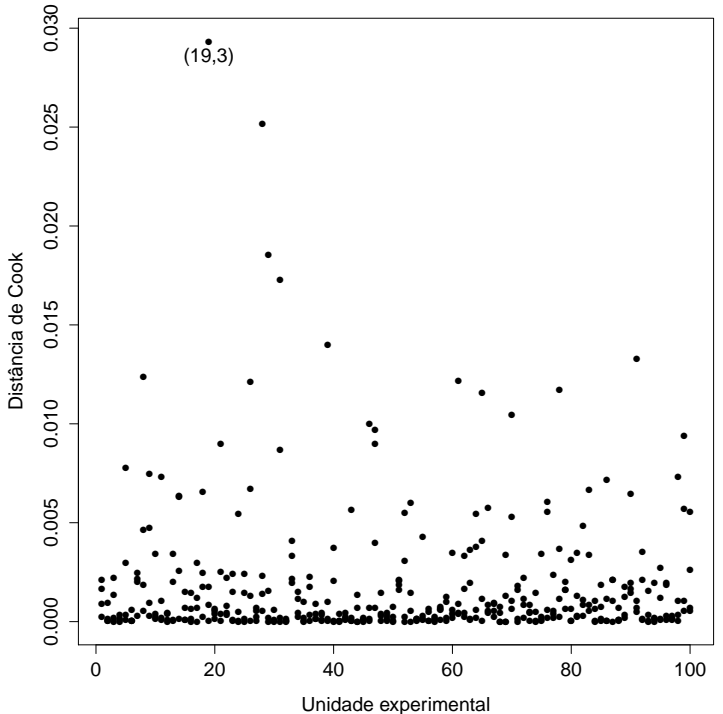

Figura 4.28: Gráfico das distâncias de Cook versus os índices das unidades experimentais do modelo marginal normal com estrutura de correlação uniforme - estudo 2. 


\section{Estudo 3}

No terceiro estudo de simulação, as observações foram geradas a partir de um modelo linear generalizado misto gama com intercepto aleatório e função de ligação log, mantendo a distribuição normal para os efeitos aleatórios. Nesse caso, temos

$$
\eta_{i j}=1+0,3 x_{i j}+u_{i}
$$

$\eta_{i j}=\log \mu_{i j}$ e $\phi=1$.

Foram ajustados quatro modelos: um modelo linear misto com intercepto aleatório, um modelo marginal normal com estrutura de correlação uniforme, um modelo marginal gama com função de ligação log e estrutura de correlação uniforme, e um modelo linear generalizado hierárquico gama com intercepto aleatório e distribuição normal para os efeitos aleatórios, com $v(u)=u$. Mesmo com observações geradas a partir de uma distribuição gama, modelos normais foram ajustados com o intuito de verificar o desempenho das técnicas de diagnóstico quando a distribuição assumida pelo modelo ajustado não está adequada. Em geral, nos modelos normais é assumida a função de ligação identidade $\eta_{i j}=\mu_{i j}$. Portanto, as estimativas dos parâmetros obtidas nos ajustes dos modelos normais não são comparáveis com os valores de parâmetros utilizados na simulação. Os resultados dos ajustes dos quatro modelos estão apresentados nas Tabelas 4.5 a 4.8 , respectivamente.

\begin{tabular}{crr}
\hline Parâmetro & Estimativa & Erro padrão \\
\hline$\beta_{1}$ & $-0,492$ & 2,887 \\
$\beta_{2}$ & 4,373 & 0,573 \\
$\sigma_{u}^{2}$ & 164,480 & \\
$\sigma^{2}$ & 644,840 & \\
\hline
\end{tabular}

Tabela 4.5: Estimativas (erros padrões) dos parâmetros do modelo linear misto com intercepto aleatório - estudo 3.

Para o modelo linear misto, os gráficos dos resíduos marginais e condicionais padronizados versus os índices das unidades experimentais (Figura 4.29) exibem a presença de muitos valores discrepantes. Pelos padrões observados nos gráficos dos resíduos marginais e condicionais padronizados versus os valores ajustados (Figura 4.30), percebe-se que as suposições de linearidade dos efeitos fixos e homocedasticidade dos erros condicionais estão comprometidas.

O gráfico dos resíduos para a estrutura de covariância (Figura 4.31) apresenta algumas unidades experimentais com valores muito altos, o que mostra que a estrutura de covariância adotada no modelo não está adequada. Os gráficos Q-Q dos resíduos marginais e condicionais padronizados (Figura 4.32) mostram que o modelo linear misto não está bem ajustado, indicando que a distribuição dos erros marginais e condicionais não é normal. Porém, o gráfico dos resíduos minimamente confundidos não dá indícios de que os erros condicionais possuem distribuição diferente da normal (Figura 4.33). Esse exemplo reforça o que foi dito por Schützenmeister e Piepho [2011] sobre o efeito de super normalidade que uma combinação linear pode causar. A proposta desses autores quanto a utilizar os resíduos $t_{i j}^{*}$ indica que a distribuição normal não é a mais adequada para o erro condicional (Figura 4.34). O gráfico dos resíduos quantílicos mostra que o modelo linear misto não está bem ajustado (Figura 4.35). 
Nos gráficos que envolvem a distância de Mahalanobis $\hat{\zeta}_{i}$ (Figura 4.36), algumas unidades experimentais apresentaram valores altíssimos de $\hat{\zeta}_{i}$, o que indica que são discrepantes. Mesmo com os efeitos aleatórios sendo gerados a partir de uma distribuição normal, gráfico Q-Q das distâncias de Mahalanobis não apresentou um bom ajuste do modelo. Isso indica que esse gráfico pode ser sensível a um erro em outra suposição do modelo.

As medidas de alavanca estão apresentadas nas Figuras 4.37 e 4.38. Algumas observações foram destacadas com valores altos para a distância de Cook (Figura 4.39). Isso também é consequência da distribuição inadequada utilizada no ajuste do modelo.

Para o ajuste do modelo marginal normal com estrutura de correlação uniforme (Tabela 4.6), também são detectadas observações discrepantes pelo gráfico dos resíduos padronizados e o gráfico Q-Q indica que o modelo normal não é adequado (Figura 4.40). Os gráficos de alavanca (Figura 4.41) e de influência (Figura 4.42) apresentam resultados parecidos com os obtidos no ajuste do modelo linear misto, indicando as mesmas observações como influentes.

\begin{tabular}{crrr}
\hline Parâmetro & Estimativa & EP naive & EP robusto \\
\hline$\beta_{1}$ & $-0,498$ & 2,884 & 2,219 \\
$\beta_{2}$ & 4,375 & 0,573 & 0,779 \\
$\phi^{-1}$ & 808,265 & & \\
$\alpha$ & 0,2 & & \\
\hline
\end{tabular}

Tabela 4.6: Estimativas (erros padrões) dos parâmetros do modelo marginal normal com estrutura de correlação uniforme - estudo 3.

No ajuste do modelo marginal gama, as estimativas dos parâmetros podem ser comparadas com os valores originais dos parâmetros utilizados na simulação. Nesse caso, obtivemos uma estimativa próxima do verdadeiro valor para o coeficiente angular, e um valor um pouco maior para o intercepto (Tabela 4.7). É importante lembrar que as observações foram geradas para um modelo condicional, podendo o modelo marginal não ser o mais adequado nesse caso.

\begin{tabular}{crrr}
\hline Parâmetro & Estimativa & EP naive & EP robusto \\
\hline$\beta_{1}$ & 1,444 & 0,175 & 0,226 \\
$\beta_{2}$ & 0,289 & 0,322 & 0,037 \\
$\phi^{-1}$ & 2,931 & & \\
$\alpha$ & 0,333 & & \\
\hline
\end{tabular}

Tabela 4.7: Estimativas (erros padrões) dos parâmetros do modelo marginal gama com estrutura de correlação uniforme - estudo 3.

O gráfico dos resíduos padronizados indica a presença de observações discrepantes, e o gráfico Q-Q apresenta pequenos sinais de inadequação do ajuste do modelo (Figura 4.43), mas o resultado é melhor do que aquele obtido anteriormente no modelo normal (Figura 4.40). O gráfico com as medidas de alavanca (Figura 4.44) apresenta valores próximos daqueles obtidos nos outros ajustes, e o gráfico das distâncias de Cook (Figura 4.45) indica a observação 3 da unidade experimental 56 como influente e também indica a observação 4 da unidade experimental 81. A remoção dessas observações resulta em $\hat{\beta}_{1}=1,225$ e $\hat{\beta}_{2}=0,327$, com $\hat{\phi}^{-1}=2,203$ e $\hat{\alpha}=0,295$.

Finalmente, é ajustado o modelo linear generalizado hierárquico gama com efeitos aleatórios 
normais e $\eta_{i j}=\log \mu_{i j}$ (Tabela 4.8).

\begin{tabular}{crr}
\hline Parâmetro & Estimativa & Erro padrão \\
\hline$\beta_{1}$ & 0,804 & 0,149 \\
$\beta_{2}$ & 0,311 & 0,025 \\
$\sigma_{u}^{2}$ & 1,005 & \\
$\phi^{-1}$ & 1,128 & \\
\hline
\end{tabular}

Tabela 4.8: Estimativas (erros padrões) dos parâmetros do modelo linear generalizado hierárquico gama com intercepto aleatório - estudo 3.

Nesse caso, os resíduos utilizados são os componentes do desvio padronizados. As Figuras 4.46 a 4.47 indicam que o modelo está adequado. As medidas de alavanca baseadas na matriz $\boldsymbol{H}^{a}$ estão apresentadas na Figura 4.49, sem nenhuma observação se destacando por apresentar valor muito alto.
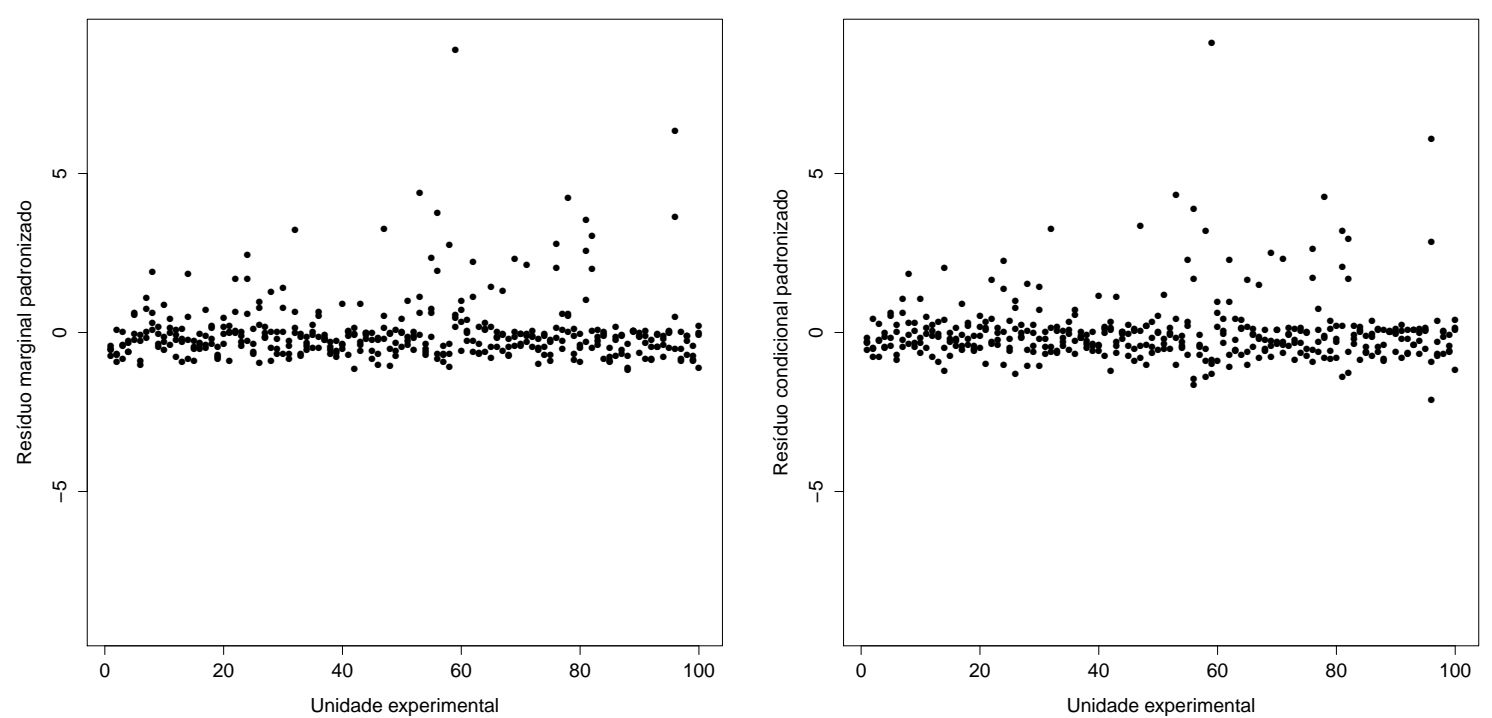

Figura 4.29: Resíduos marginais e condicionais padronizados versus os índices das unidades experimentais do modelo linear misto com intercepto aleatório - estudo 3. 

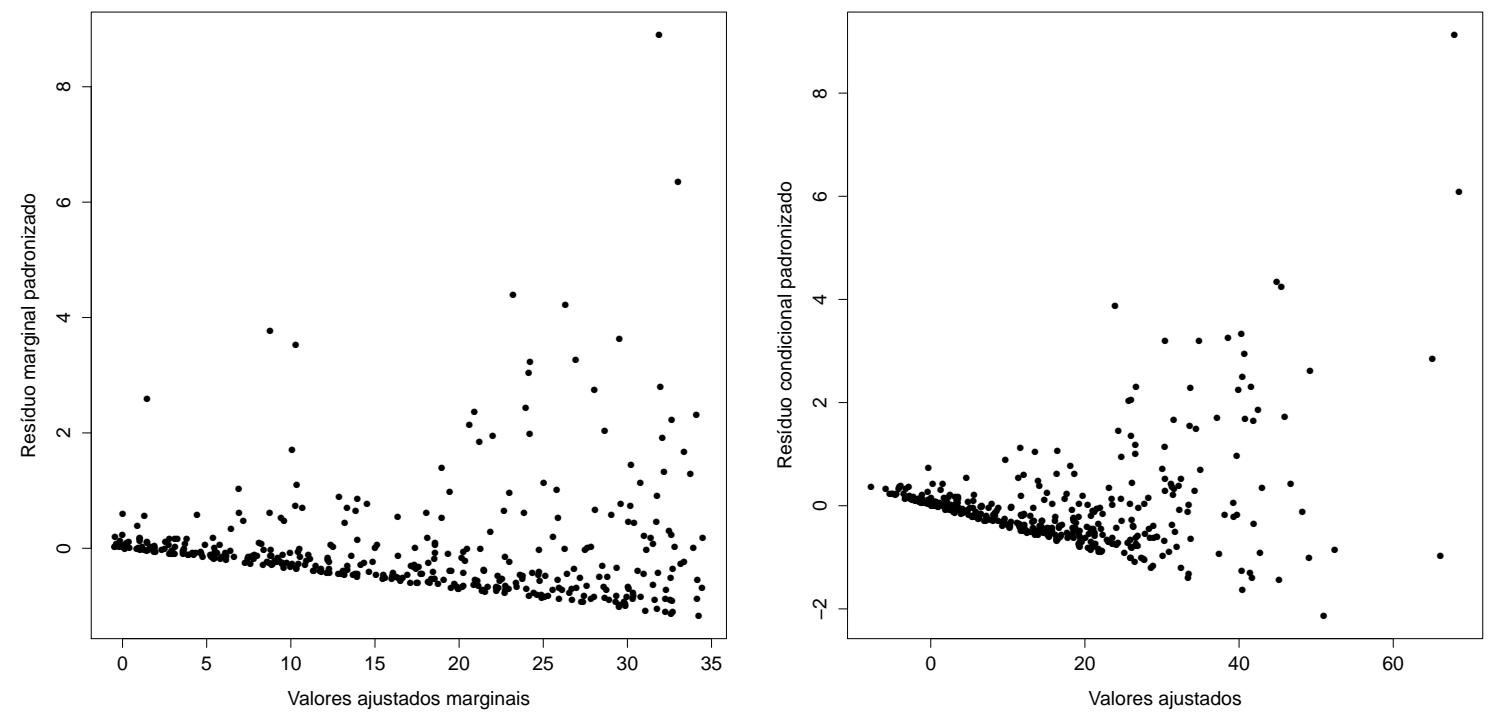

Figura 4.30: Resíduos marginais e condicionais padronizados versus os valores ajustados do modelo linear misto com intercepto aleatório - estudo 3.

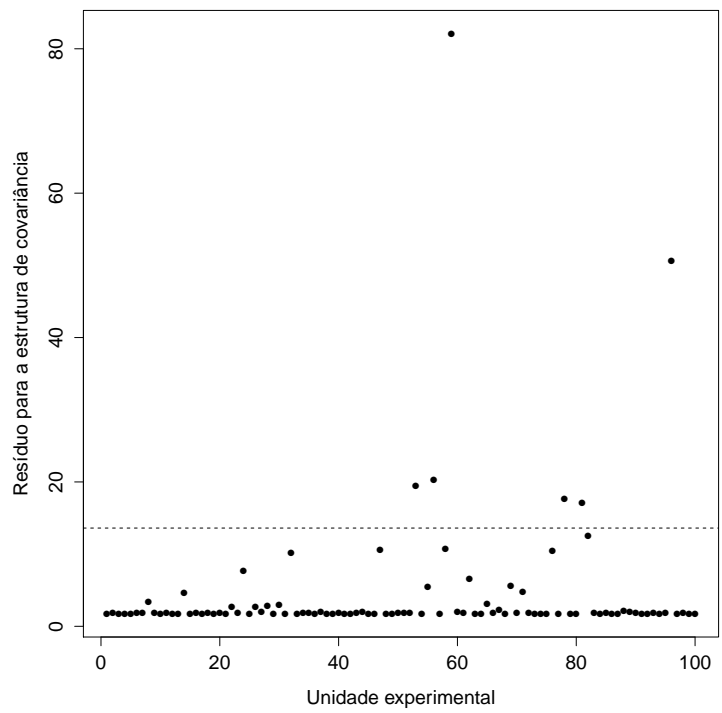

Figura 4.31: Gráfico dos resíduos para a estrutura de covariância versus os índices das unidades experimentais do modelo linear misto com intercepto aleatório - estudo 3. 

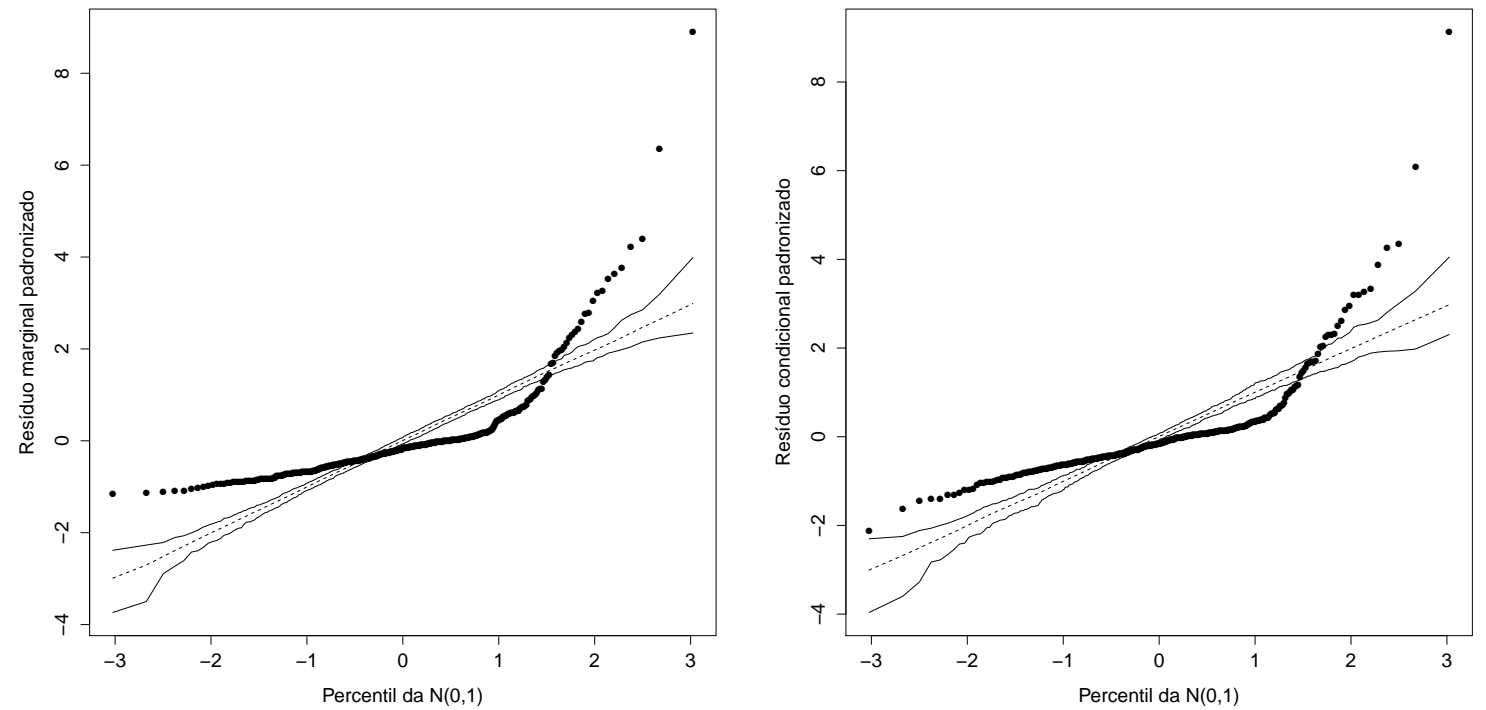

Figura 4.32: Gráficos $Q-Q$ dos resíduos marginais e condicionais padronizados do modelo linear misto com intercepto aleatório - estudo 3.

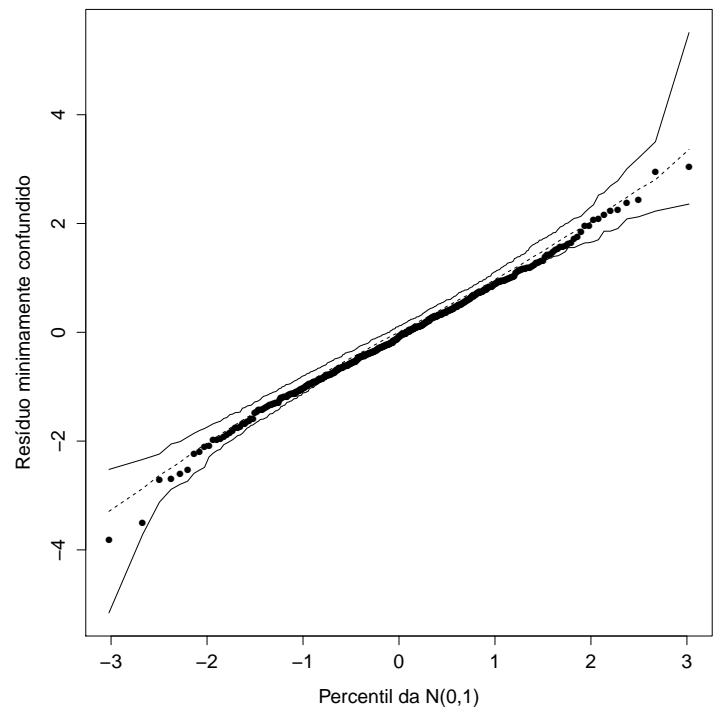

Figura 4.33: Gráfico $Q-Q$ dos resíduos minimamente confundidos do modelo linear misto com intercepto aleatório - estudo 3. 


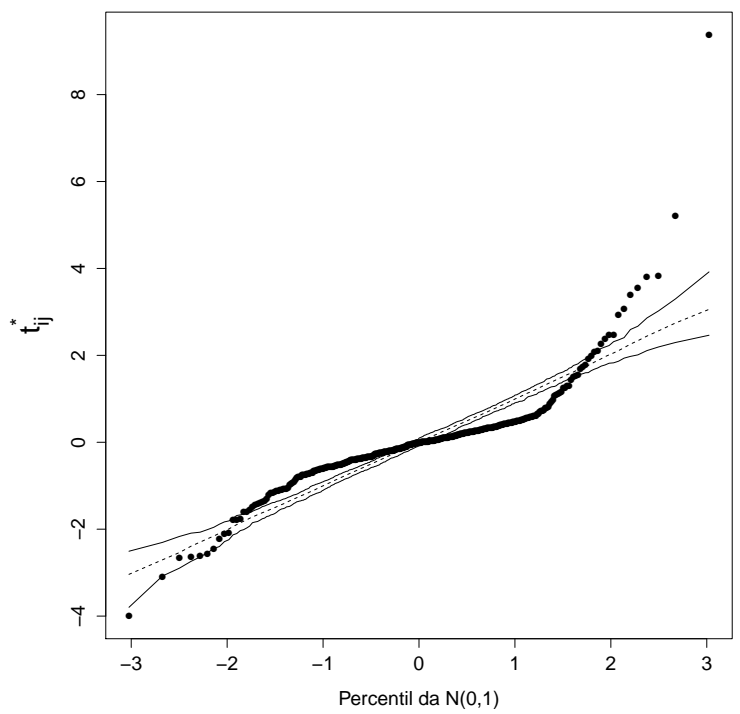

Figura 4.34: Gráfico $Q-Q$ dos resíduos $t_{i j}^{*}$ do modelo linear misto com intercepto aleatório - estudo 3.

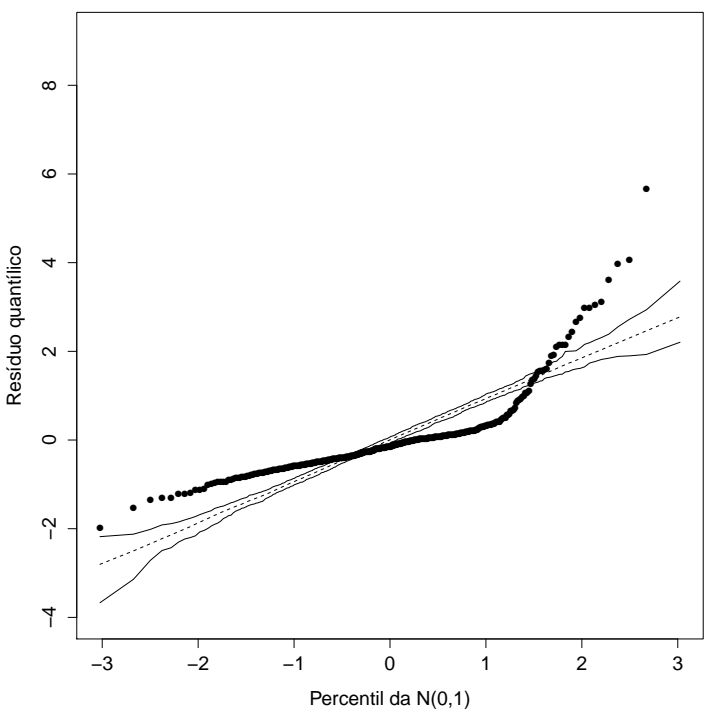

Figura 4.35: Gráfico $Q-Q$ dos resíduos quantílicos do modelo linear misto com intercepto aleatório estudo 3 . 

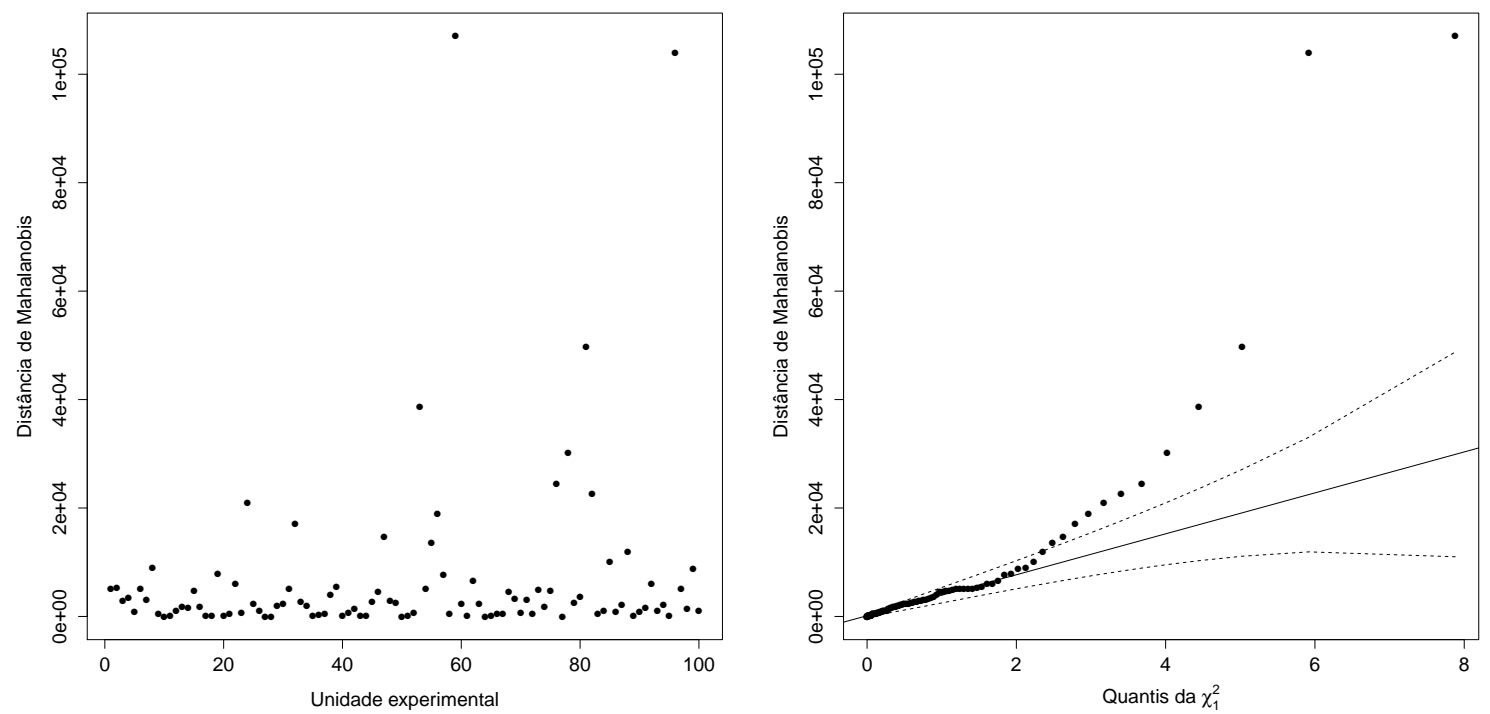

Figura 4.36: Gráficos das distâncias de Mahalanobis do modelo linear misto com intercepto aleatório estudo 3.
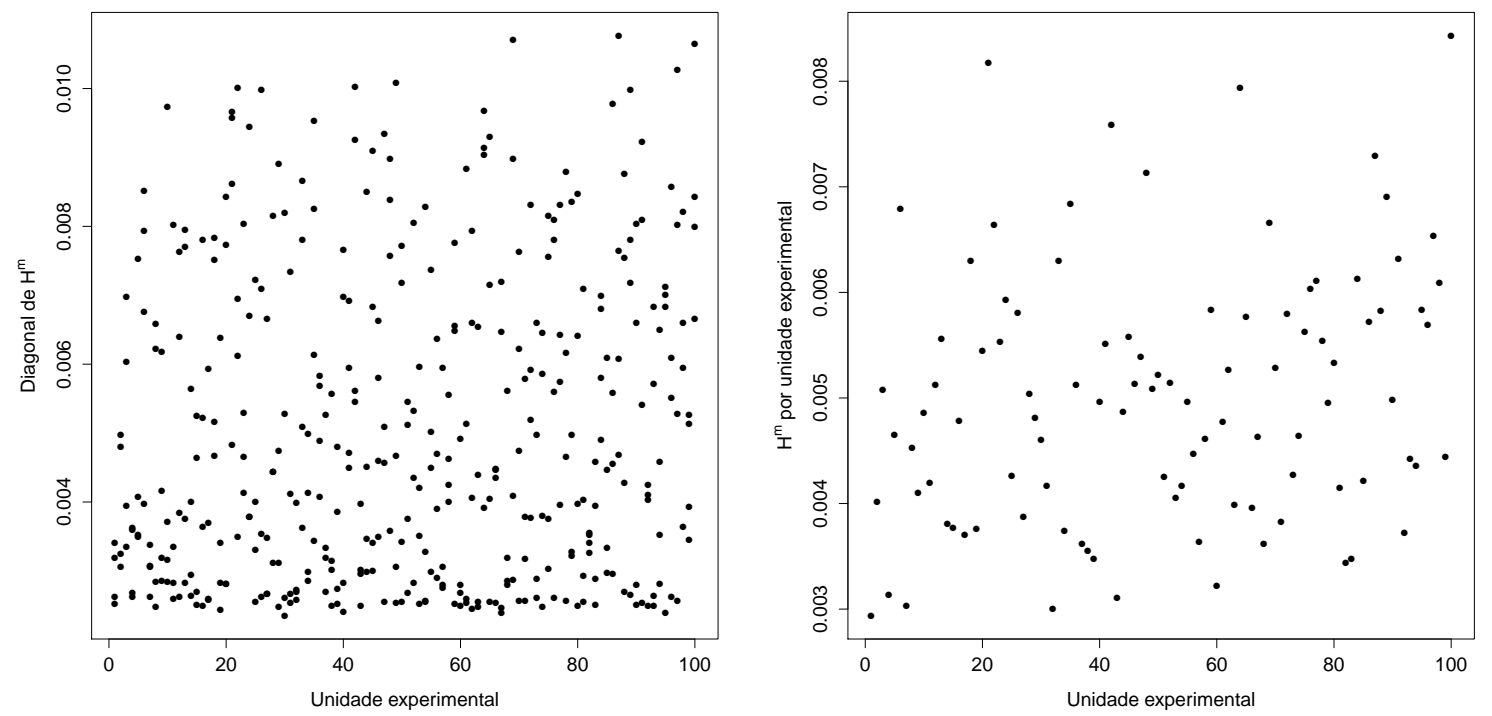

Figura 4.37: Gráficos das medidas de alavanca com base em $\boldsymbol{H}^{m}$ versus os índices das unidades experimentais do modelo linear misto com intercepto aleatório - estudo 3. 

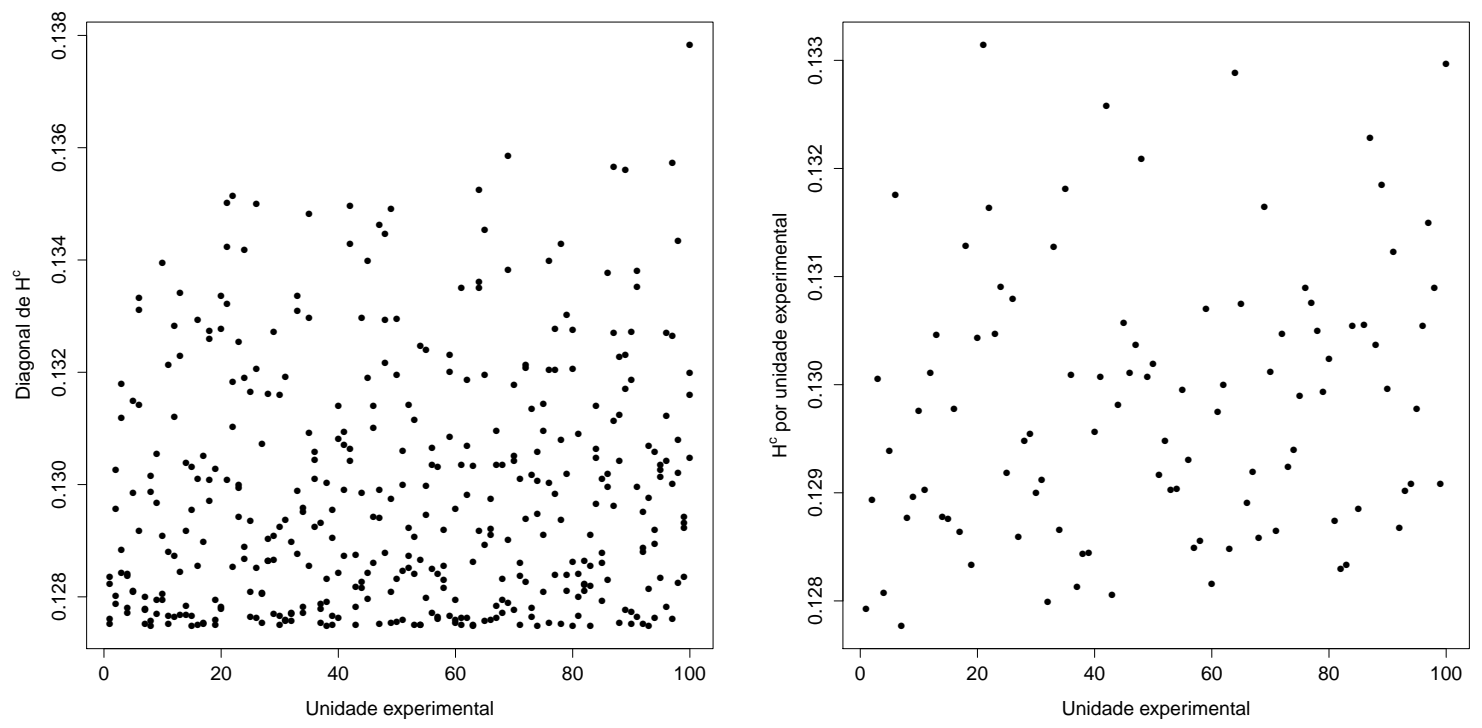

Figura 4.38: Gráficos das medidas de alavanca com base em $\boldsymbol{H}^{c}$ versus os índices das unidades experimentais do modelo linear misto com intercepto aleatório - estudo 3.

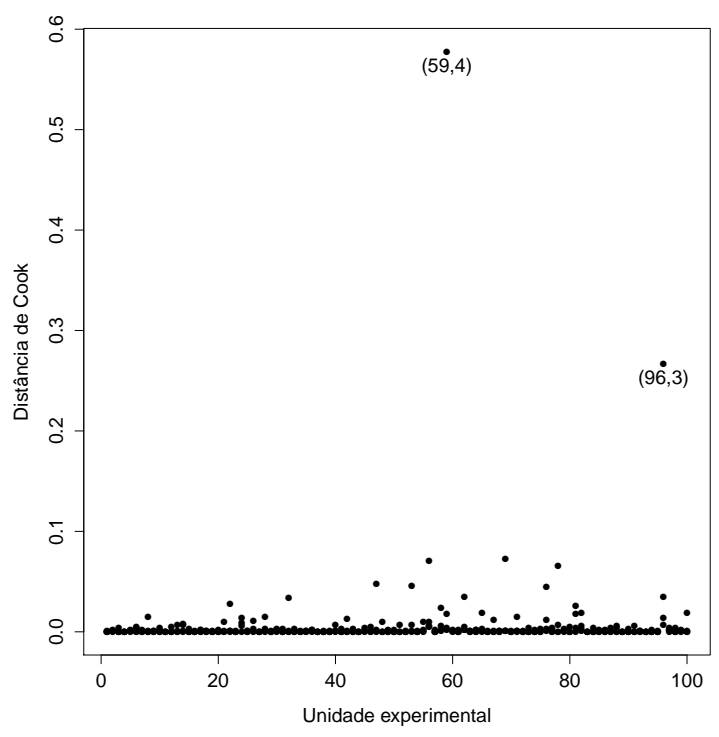

Figura 4.39: Gráfico das distâncias de Cook versus os índices das unidades experimentais do modelo linear misto com intercepto aleatório - estudo 3. 

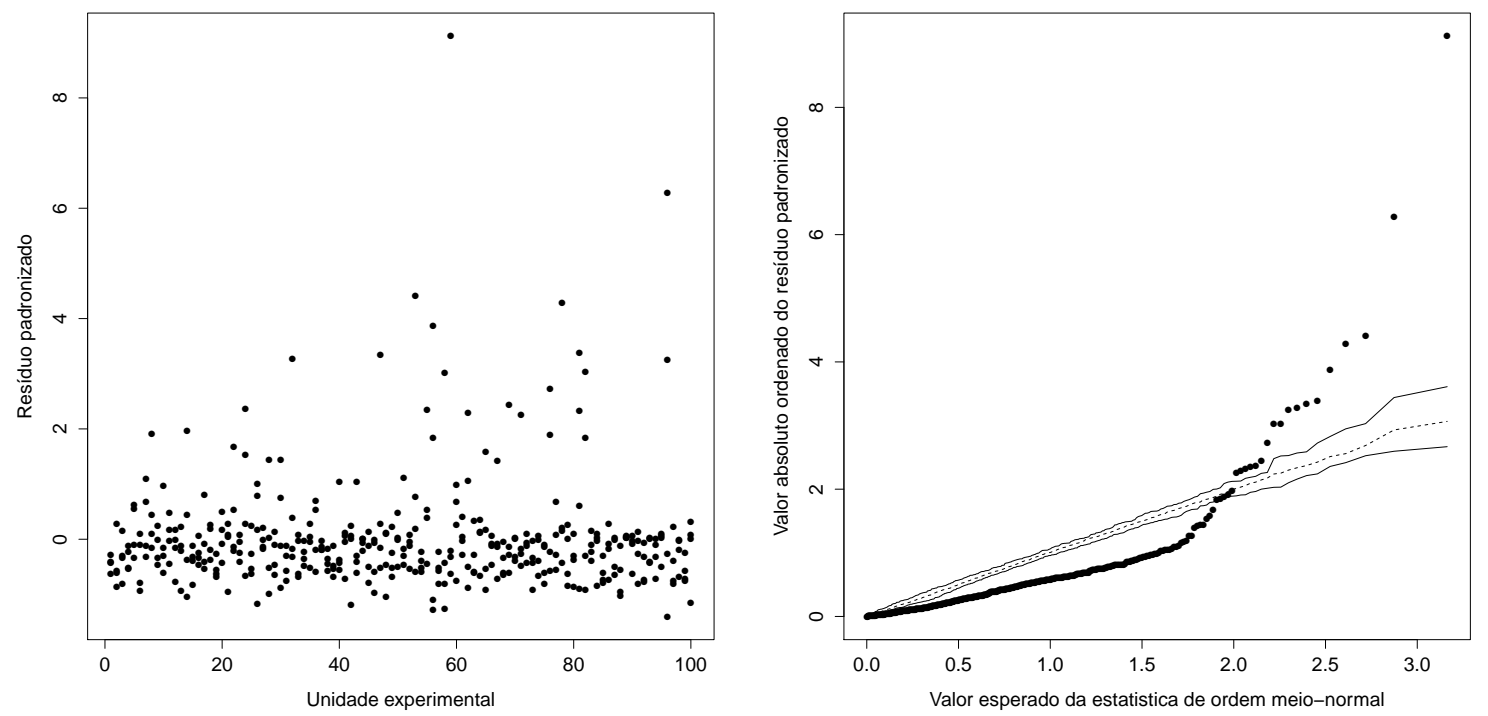

Figura 4.40: Gráficos dos resíduos padronizados do modelo marginal normal com estrutura de correlação uniforme - estudo 3.
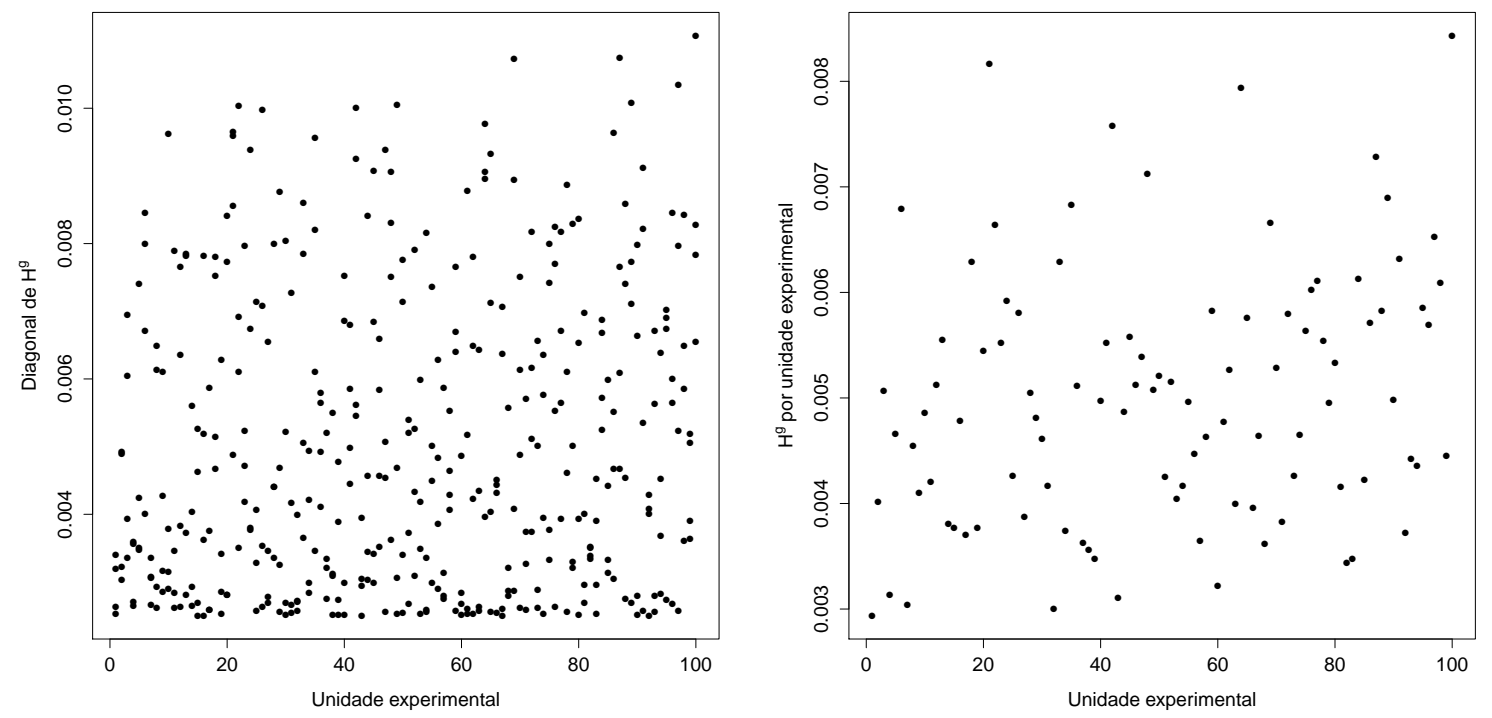

Figura 4.41: Gráficos das medidas de alavanca com base em $\boldsymbol{H}^{g}$ versus os índices das unidades experimentais do modelo marginal normal com estrutura de correlação uniforme - estudo 3. 


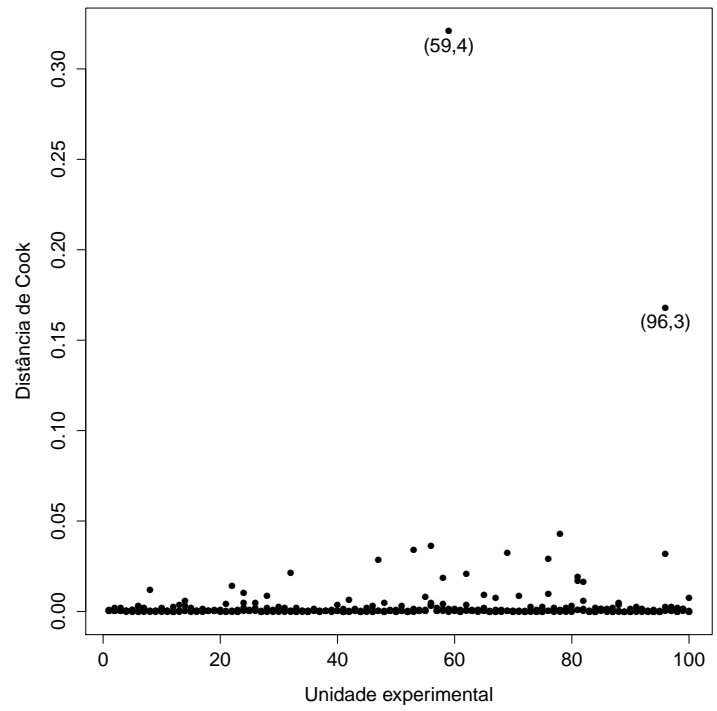

Figura 4.42: Gráfico das distâncias de Cook versus os índices das unidades experimentais do modelo marginal normal com estrutura de correlação uniforme - estudo 3.
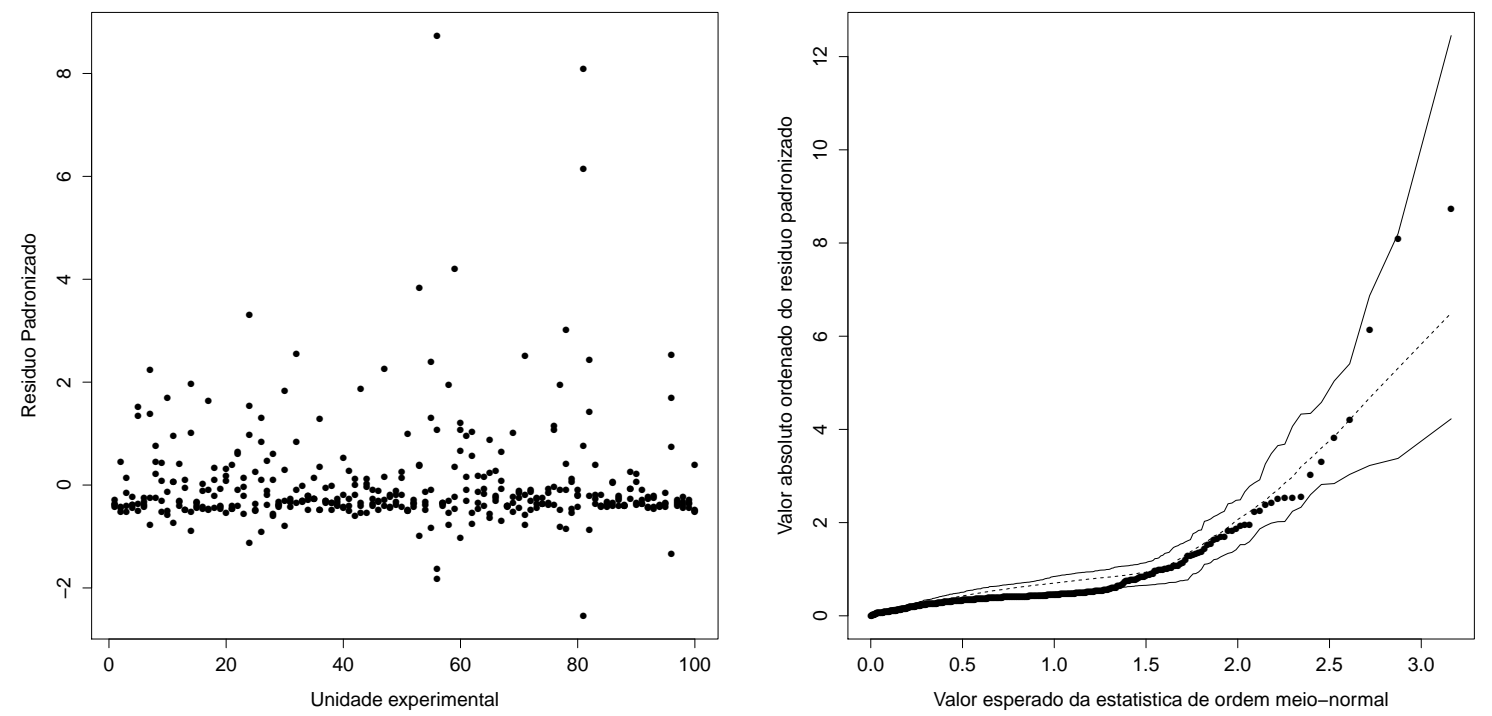

Figura 4.43: Gráficos dos resíduos padronizados do modelo marginal gama com estrutura de correlação uniforme - estudo 3. 

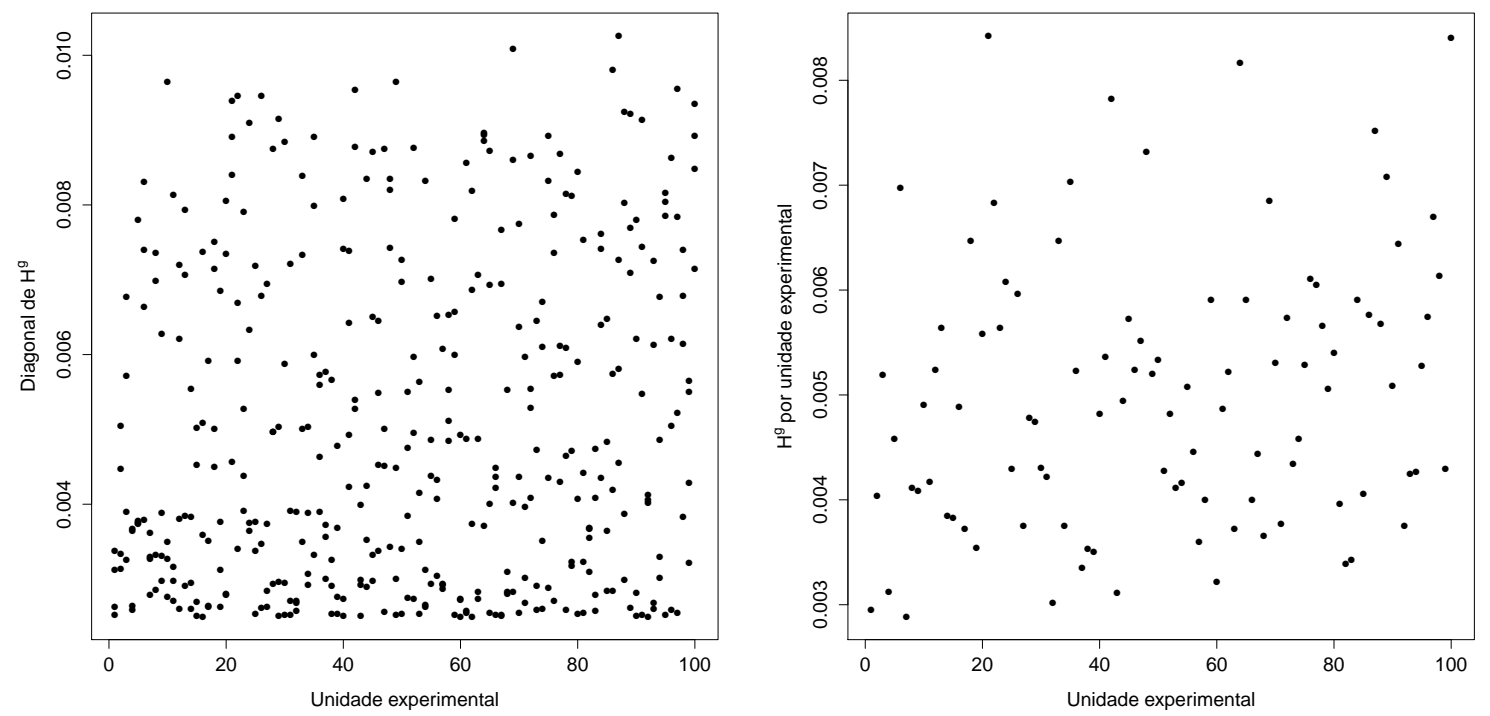

Figura 4.44: Gráficos das medidas de alavanca com base em $\boldsymbol{H}^{g}$ versus os índices das unidades experimentais do modelo marginal gama com estrutura de correlação uniforme - estudo 3.

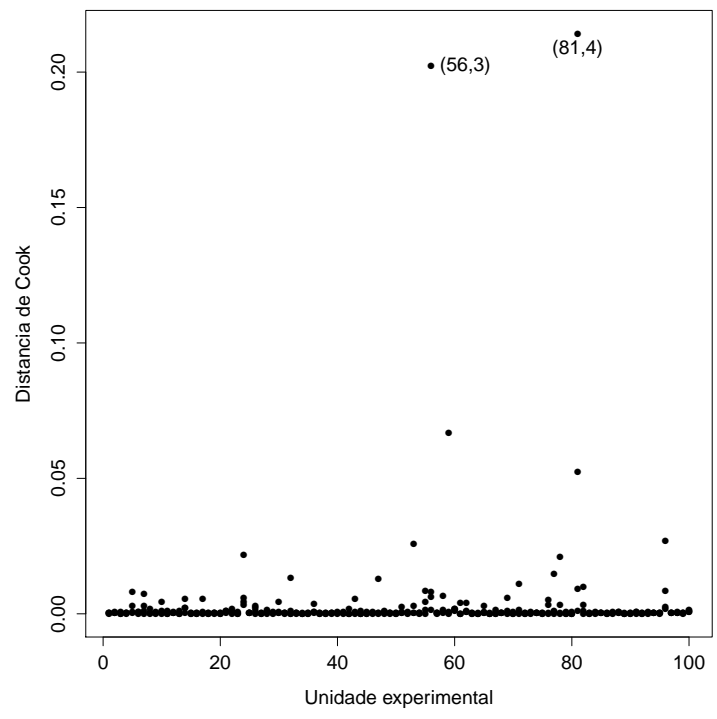

Figura 4.45: Gráfico das distâncias de Cook versus os índices das unidades experimentais do modelo marginal gama com estrutura de correlação uniforme - estudo 3. 

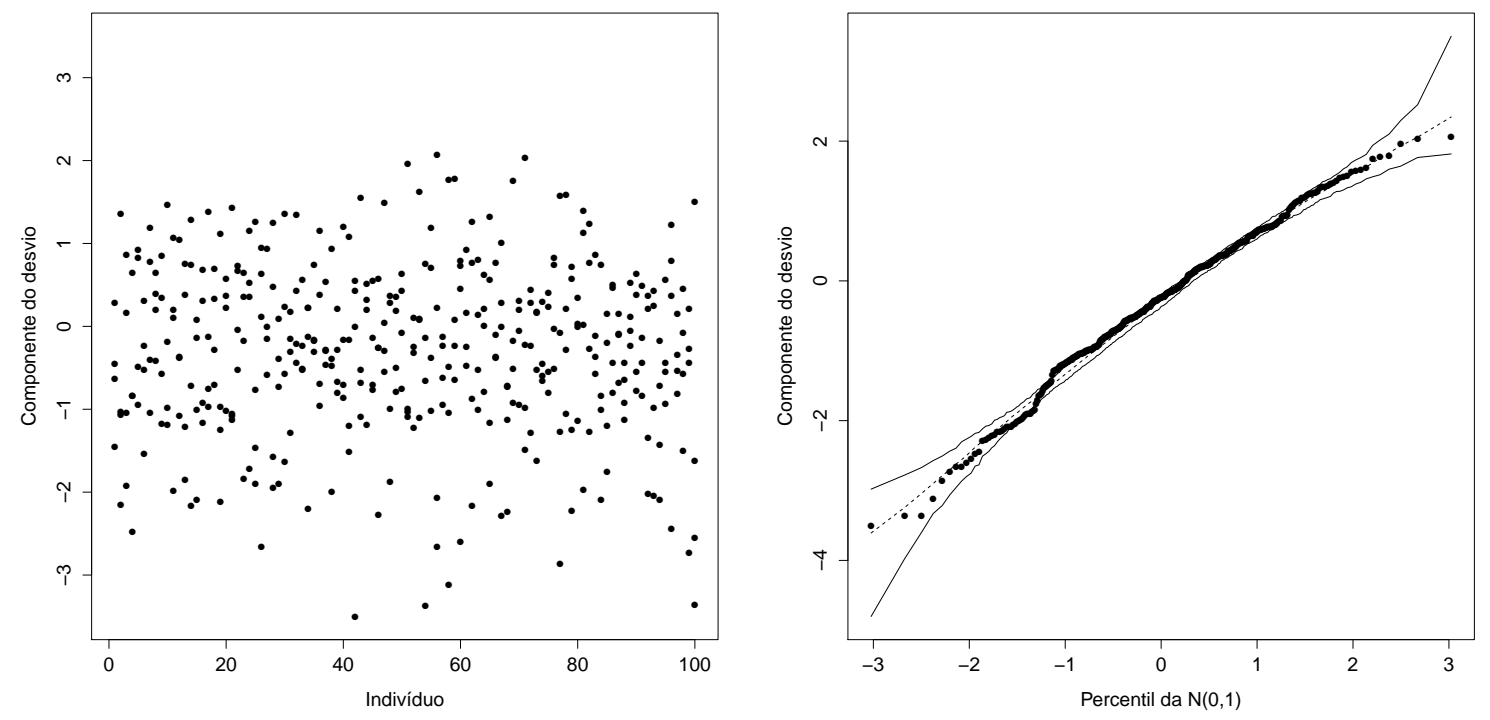

Figura 4.46: Gráficos dos componentes do desvio padronizados do modelo linear generalizado hierárquico gama com intercepto aleatório - estudo 3.

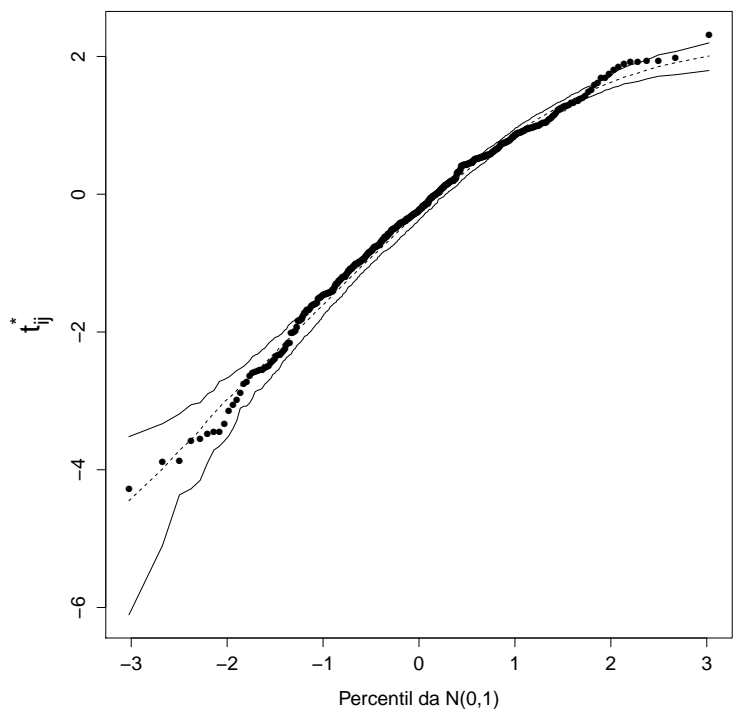

Figura 4.47: Gráfico $Q-Q$ dos resíduos $t_{i j}^{*}$ do modelo linear generalizado hierárquico gama com intercepto aleatório - estudo 3. 


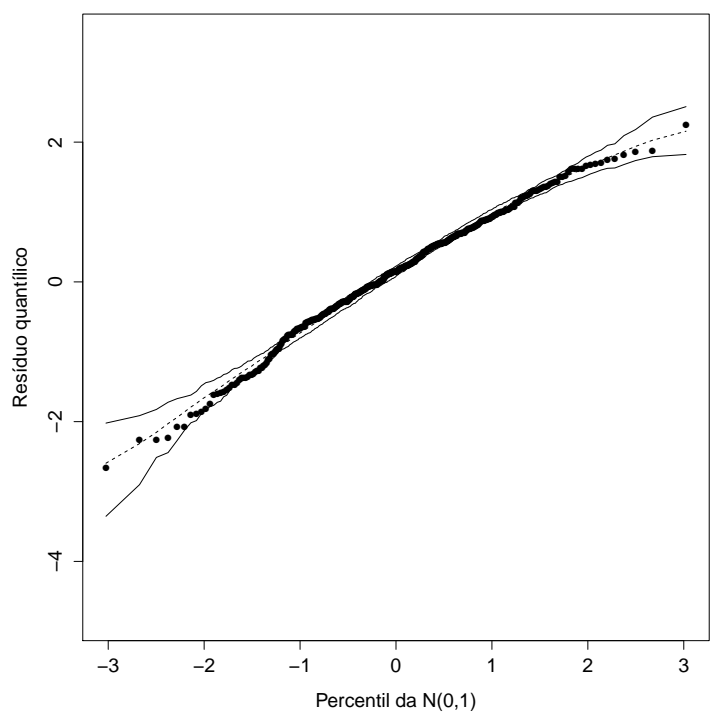

Figura 4.48: Gráfico $Q-Q$ dos resíduos quantílicos do modelo linear generalizado hierárquico gama com intercepto aleatório - estudo 3.
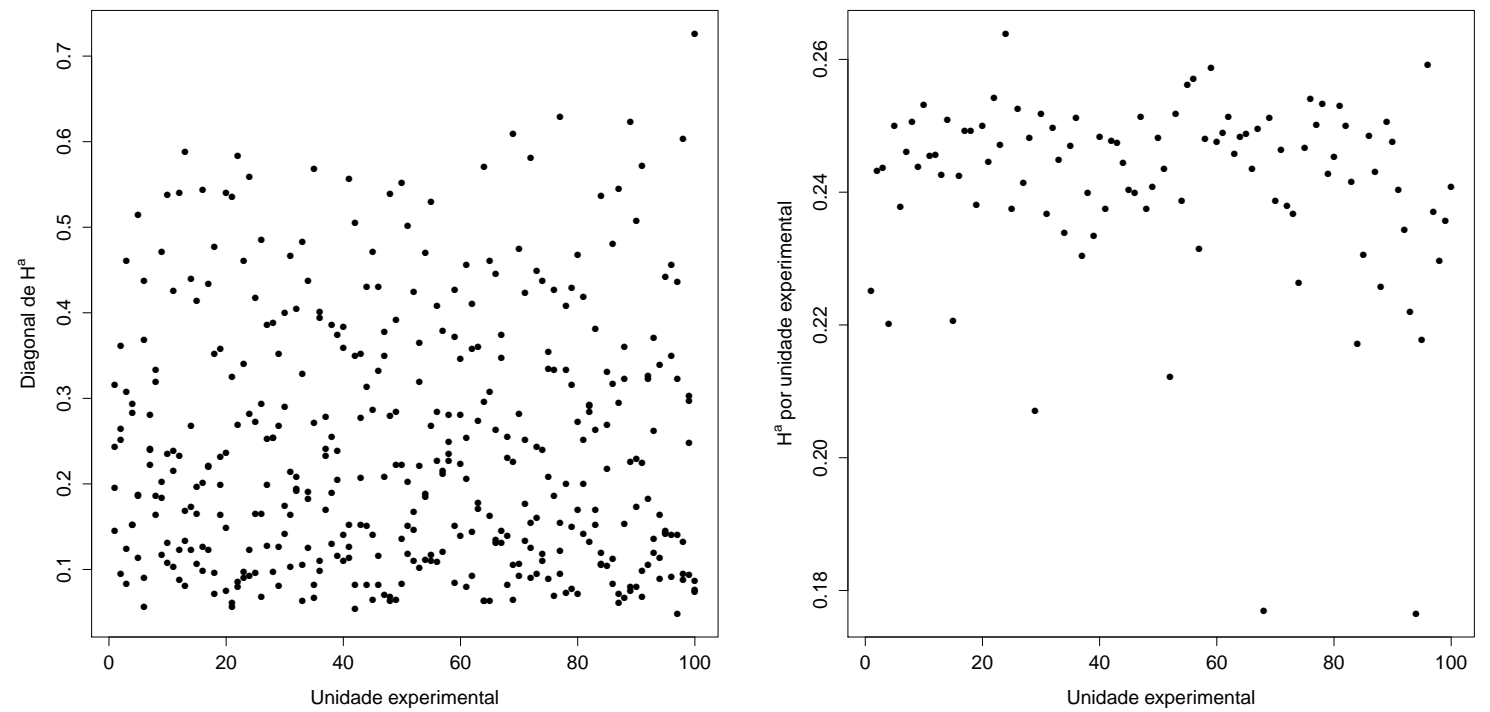

Figura 4.49: Gráficos das medidas de alavanca com base em $\boldsymbol{H}^{a}$ versus os índices das unidades experimentais do modelo linear generalizado hierárquico gama com intercepto aleatório - estudo 3. 


\subsection{Modelos com inclinação aleatória}

Neste estudo, as observações $y_{i j}$ foram geradas a partir do modelo linear misto dado em (2.3), considerando $\beta_{1}=1, \beta_{2}=2, u_{1 i} \sim N(0,1)$ independentes, $u_{2 i} \sim N(0,1)$ independentes, $\varepsilon_{i j} \sim N(0,1)$ independentes e $x_{i j} \sim U[0,8], i=1, \ldots, 100, j=1, \ldots, 4$, com $u_{1 i}, u_{2 i}$ e $\varepsilon_{i j}$ independentes.

Foram ajustados três modelos: um modelo linear misto com intercepto aleatório, um modelo linear misto com intercepto e inclinação aleatórios, e um modelo marginal com matriz de correlações de trabalho não estruturada. Os resultados desses ajustes estão apresentados nas Tabelas 4.9 a 4.11 , respectivamente.

O modelo linear misto com apenas o intercepto aleatório (Tabela 4.9) é um modelo homocedástico, enquanto os dados foram obtidos a partir de um modelo heterocedástico, como foi visto em (2.4). A estimativa do intercepto obtida é um pouco maior do que o seu valor original, enquanto o coeficiente angular está mais próximo do valor original. As estimativas dos parâmetros associados às variâncias são muito grandes.

\begin{tabular}{crr}
\hline Parâmetro & Estimativa & Erro padrão \\
\hline$\beta_{1}$ & 1,356 & 0,526 \\
$\beta_{2}$ & 1,936 & 0,064 \\
$\sigma_{u}^{2}$ & 19,855 & \\
$\sigma^{2}$ & 6,899 & \\
\hline
\end{tabular}

Tabela 4.9: Estimativas (erros padrões) dos parâmetros do modelo com intercepto aleatório para a simulação com inclinação aleatória.

A Figura 4.50 apresenta os gráficos dos resíduos marginais e condicionais padronizados versus os índices das unidades experimentais do modelo ajustado. É possível notar a presença de observações discrepantes em ambos os gráficos. Quando são feitos os gráficos dos resíduos marginais e condicionais contra os valores ajustados, é possível perceber que os pontos não estão distribuídos de maneira aleatória ao redor do valor zero. Portanto, isso indica que as suposições de linearidade dos efeitos fixos e homocedasticidade dos erros condicionais não são satisfeitas (Figura 4.51).

Fazendo o gráfico dos resíduos para a estrutura de covariância, nota-se que a estrutura não está adequada, pois há diversos valores altos para muitas unidades experimentais (Figura 4.52).

Nos gráficos do tipo Q-Q, tanto os resíduos marginais quanto os condicionais não indicam um bom ajuste (Figura 4.53), enquanto o gráfico dos resíduos minimamente confundidos apresenta um bom resultado (Figura 4.54).

Os gráficos Q-Q dos resíduos $t_{i j}^{*}$ (Figura 4.55) e dos resíduos quantílicos (Figura 4.56) também não indicam um bom ajuste.

A Figura 4.57 apresenta os gráficos das distâncias de Mahalanobis $\hat{\zeta}_{i}$ das unidades experimentais. Nesse caso, algumas unidades experimentais apresentaram valores altos, podendo ser consideradas discrepantes. O gráfico Q-Q apresentou alguns pontos fora da faixa esperada.

As medidas de alavanca estão apresentadas nas Figuras 4.58 e 4.59. No gráfico das distâncias de Cook (Figura 4.60), a observação 1 da unidade experimental 9 é destacada como o maior valor. Ajustando o modelo sem essa observação, obtemos $\hat{\beta}_{1}=1,249, \hat{\beta}_{2}=1,973, \hat{\sigma}^{2}=6,418$ e 
$\hat{\sigma}_{u}^{2}=19,429$, o que não caracteriza uma grande mudança nas estimativas obtidas anteriormente.

Quando é ajustado o modelo linear misto com intercepto e inclinação aleatórios, são obtidas estimativas mais próximas dos valores reais utilizados na simulação (Tabela 4.10). Os gráficos

\begin{tabular}{crr}
\hline Parâmetro & Estimativa & Erro padrão \\
\hline$\beta_{1}$ & 1,171 & 0,146 \\
$\beta_{2}$ & 1,996 & 0,107 \\
$\sigma_{u 1}^{2}$ & 0,985 & \\
$\sigma_{u 2}^{2}$ & 1,078 & \\
$\sigma^{2}$ & 0,889 & \\
\hline
\end{tabular}

Tabela 4.10: Estimativas (erros padrões) dos parâmetros do modelo com intercepto e inclinação aleatórios.

dos resíduos marginais e condicionais padronizados versus os índices das unidades experimentais não detectam nenhuma observação discrepante (Figura 4.61). Os gráficos desses resíduos versus os valores ajustados (Figura 4.62) não apresentam nenhum padrão parecido com o que foi obtido no modelo anterior (Figura 4.51), indicando a linearidade dos efeitos fixos e a homocedasticidade dos erros condicionais.

No gráfico dos resíduos para a estrutura de covariância (Figura 4.63), não são destacados valores muito altos para as unidades experimentais, o que indica que a estrutura do modelo ajustado está adequada. Nessa situação, todos os diferentes resíduos utilizados em gráficos do tipo Q-Q confirmam o bom ajuste do modelo (Figuras 4.64 a 4.67). O gráfico das distâncias de Mahalanobis das unidades experimentais não destaca valores altos, e o gráfico Q-Q apresenta os pontos dentro da faixa esperada (Figura 4.68).

As Figuras 4.69 e 4.70 apresentam os gráficos com as medidas de alavanca, e a Figura 4.71 apresenta o gráfico de influência. O maior valor da distância de Cook está na observação 2 da unidade experimental 100. Eliminando essa observação, as estimativas são $\hat{\beta}_{1}=1,142$ e $\hat{\beta}_{2}=2,005$, com $\hat{\sigma}^{2}=0,882, \hat{\sigma}_{u 1}^{2}=0,863$ e $\hat{\sigma}_{u 2}^{2}=1,076$ o que não caracteriza essa observação como influente. Repare que o valor da distância de Cook nesse caso é bem menor do que aquele obtido quando é ajustado o modelo apenas com o intercepto aleatório (Figura 4.60).

No ajuste do modelo marginal normal com matriz de correlações de trabalho não estruturada, as estimativas dos parâmetros $\boldsymbol{\beta}$ são próximas dos verdadeiros valores (Tabela 4.11), mas esse modelo considera a mesma estrutura de covariância para todas as unidades experimentais, o que não está de acordo com o modelo utilizado na geração dos dados.

\begin{tabular}{crrr}
\hline Parâmetro & Estimativa & EP naive & EP robusto \\
\hline$\beta_{1}$ & 1,206 & 0,502 & 0,306 \\
$\beta_{2}$ & 1,968 & 0,054 & 0,124 \\
$\phi^{-1}$ & 26,66 & & \\
\hline
\end{tabular}

Tabela 4.11: Estimativas (erros padrões) dos parâmetros do modelo marginal normal com matriz de correlações não estruturada. 
A matriz de correlações de trabalho estimada é dada por

$$
\hat{\boldsymbol{R}}_{i}=\left[\begin{array}{cccc}
1 & 0,645 & 0,866 & 0,784 \\
0,645 & 1 & 0,636 & 0,645 \\
0,866 & 0,636 & 1 & 0,867 \\
0,784 & 0,645 & 0,867 & 1
\end{array}\right]
$$

O gráfico dos resíduos detecta observações discrepantes, e o gráfico Q-Q não indica um bom ajuste (Figura 4.72). A Figura 4.73 apresenta o gráfico com as medidas de alavanca, e a Figura 4.74 apresenta o gráficos com as distâncias de Cook. A observação 1 da unidade experimental 9 possui o maior valor. Sem essa observação, o ajuste resulta em $\hat{\beta}_{1}=1,06$ e $\hat{\beta}_{2}=2,017$, que são estimativas mais próximas dos valores originais utilizados na simulação.
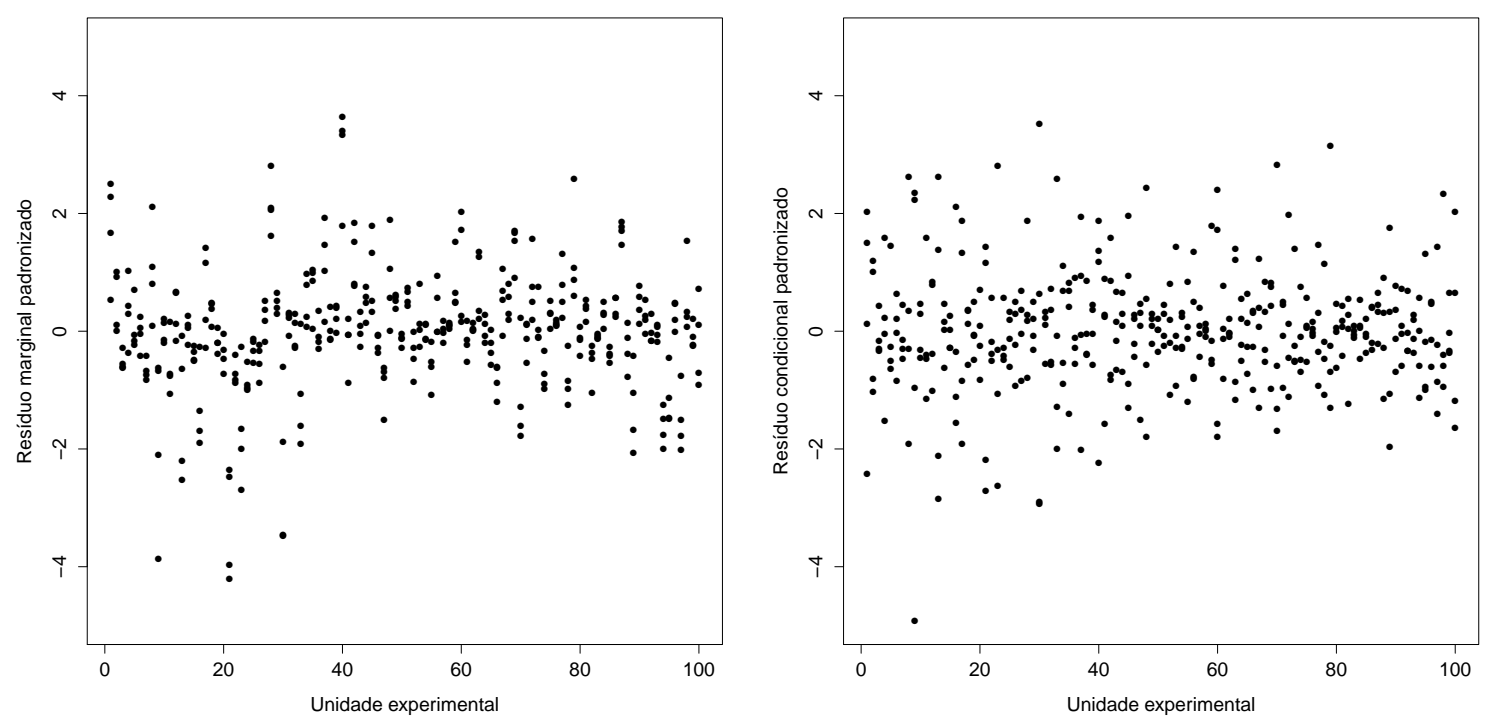

Figura 4.50: Resíduos marginais e condicionais padronizados versus os indices das unidades experimentais do modelo com intercepto aleatório para a simulação com inclinação aleatória. 

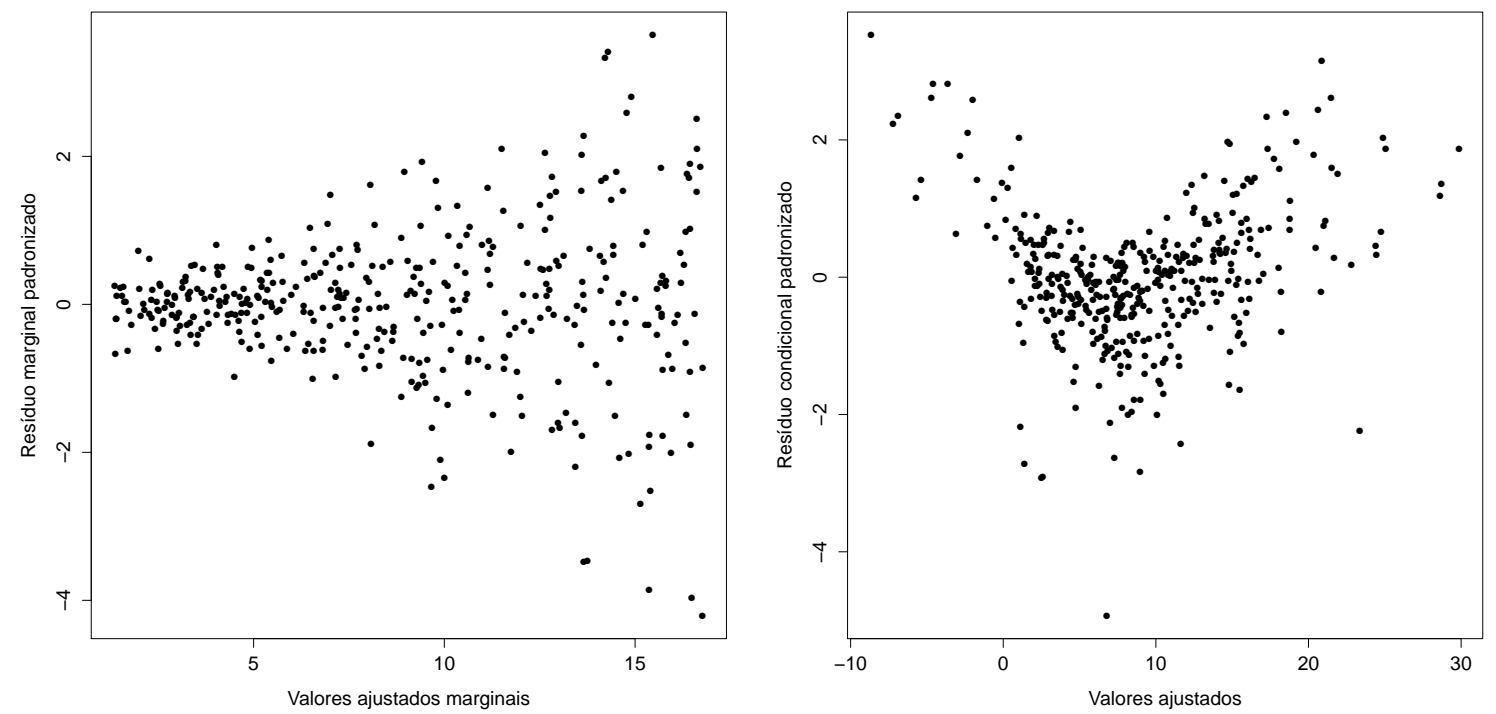

Figura 4.51: Resíduos marginais e condicionais padronizados versus os valores ajustados do modelo com intercepto aleatório para a simulação com inclinação aleatória.

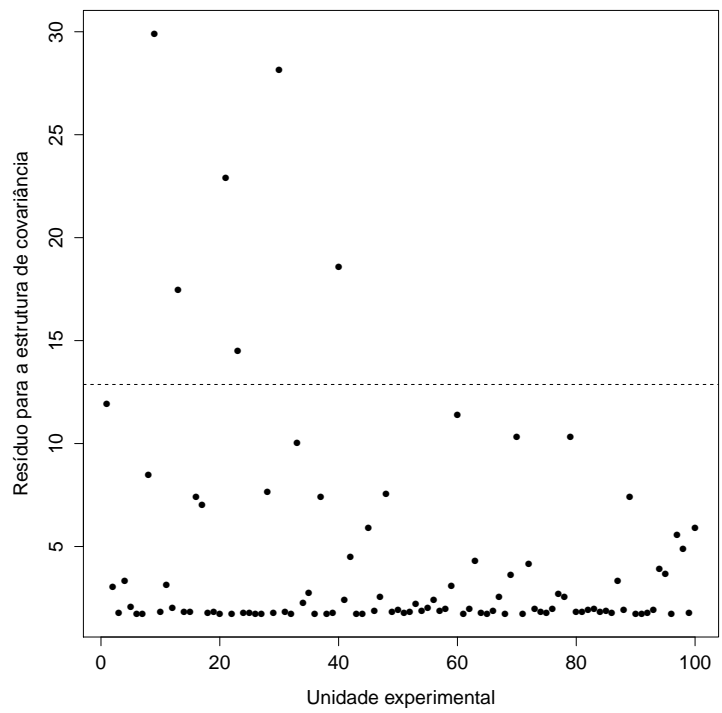

Figura 4.52: Gráfico $Q-Q$ dos resíduos para a estrutura de covariância do modelo com intercepto aleatório para a simulação com inclinação aleatória. 

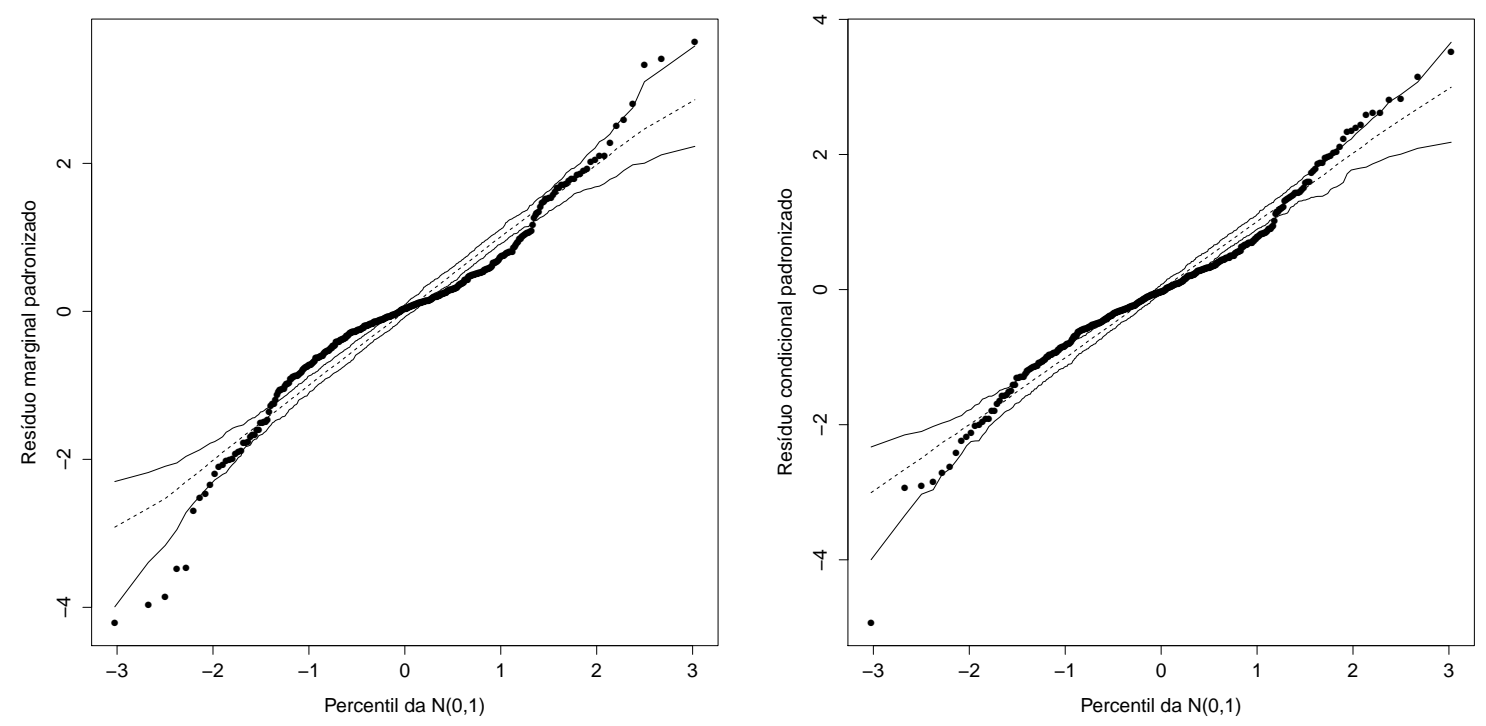

Figura 4.53: Gráficos $Q-Q$ dos resíduos marginais e condicionais padronizados do modelo com intercepto aleatório para a simulação com inclinação aleatória.

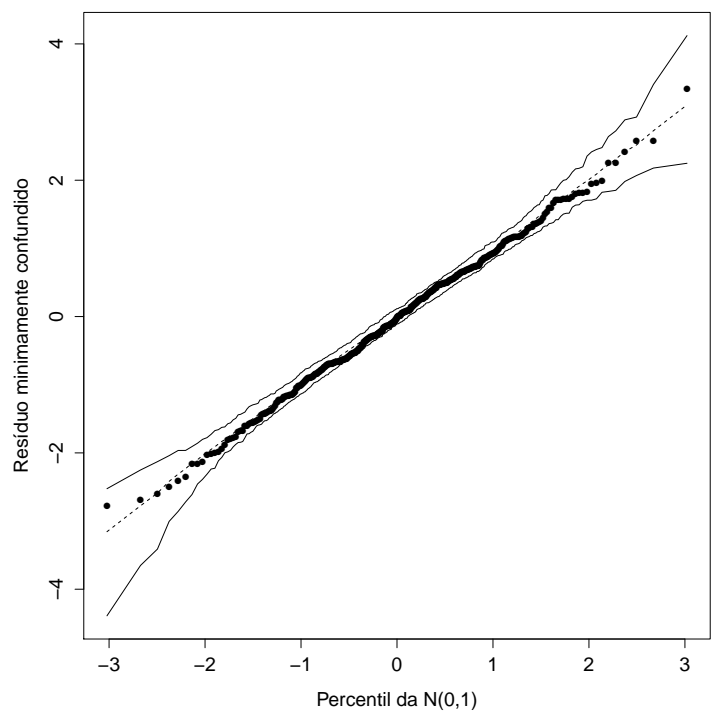

Figura 4.54: Gráfico $Q-Q$ dos resíduos minimamente confundidos do modelo com intercepto aleatório para a simulação com inclinação aleatória. 


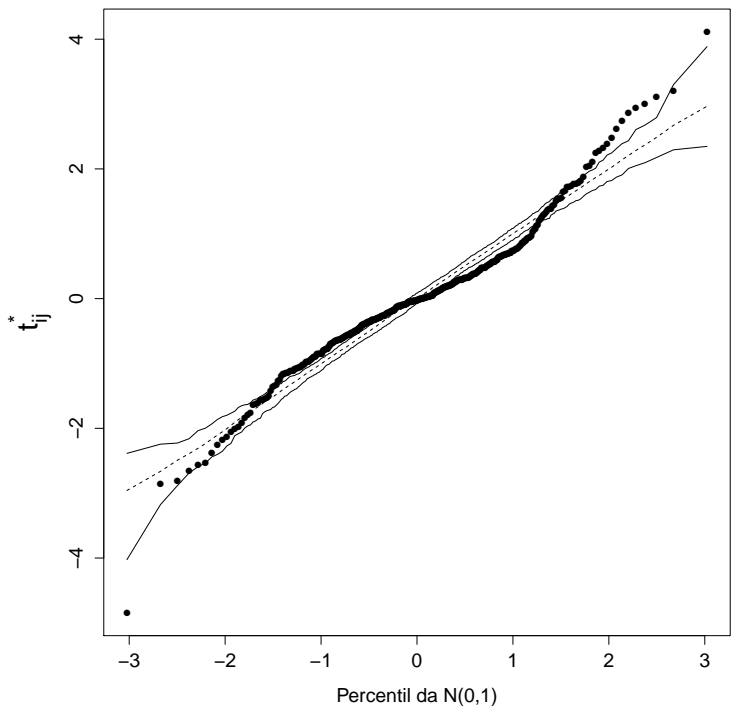

Figura 4.55: Gráfico $Q-Q$ dos resíduos $t_{i j}^{*}$ do modelo com intercepto aleatório para a simulação com inclinação aleatória.

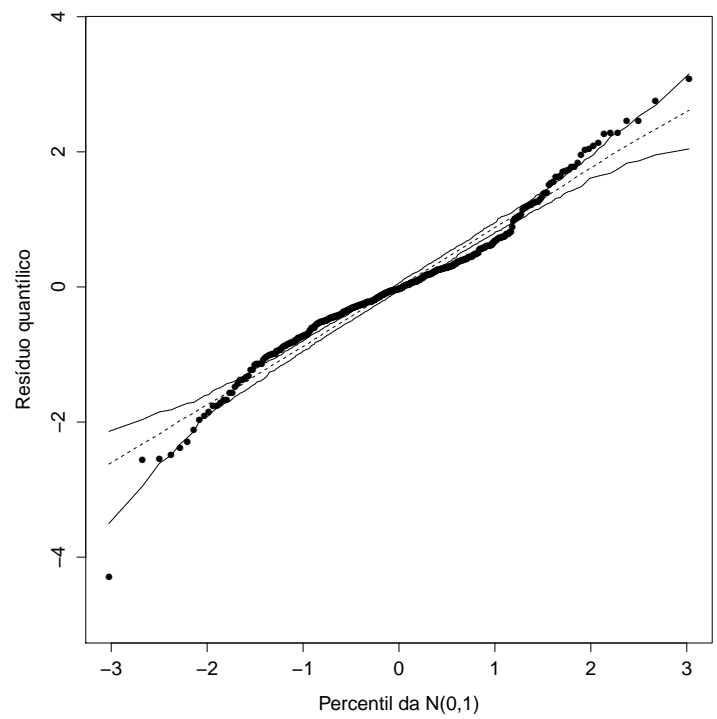

Figura 4.56: Gráfico dos resíduos quantílicos do modelo com intercepto aleatório para a simulação com inclinação aleatória. 

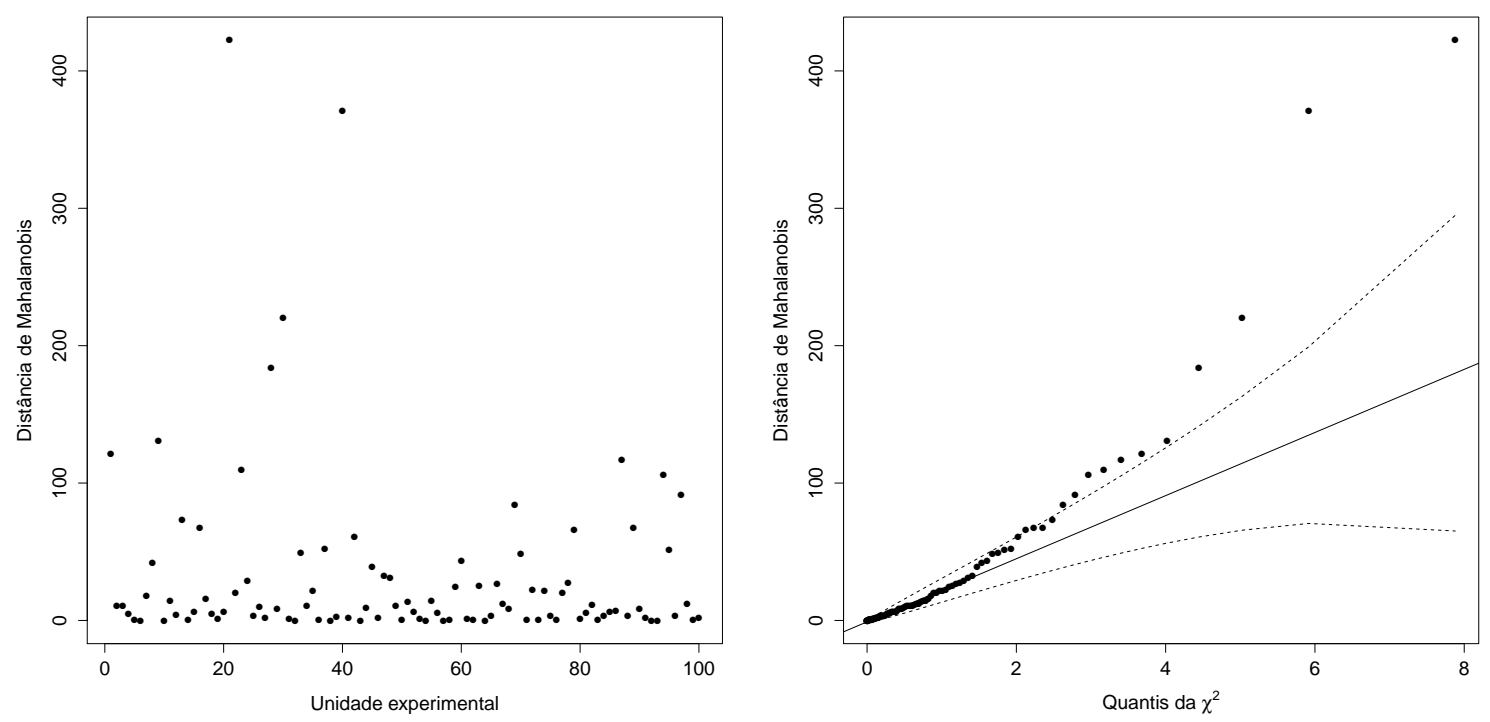

Figura 4.57: Gráficos das distâncias de Mahalanobis do modelo com intercepto aleatório para a simulação com inclinação aleatória.
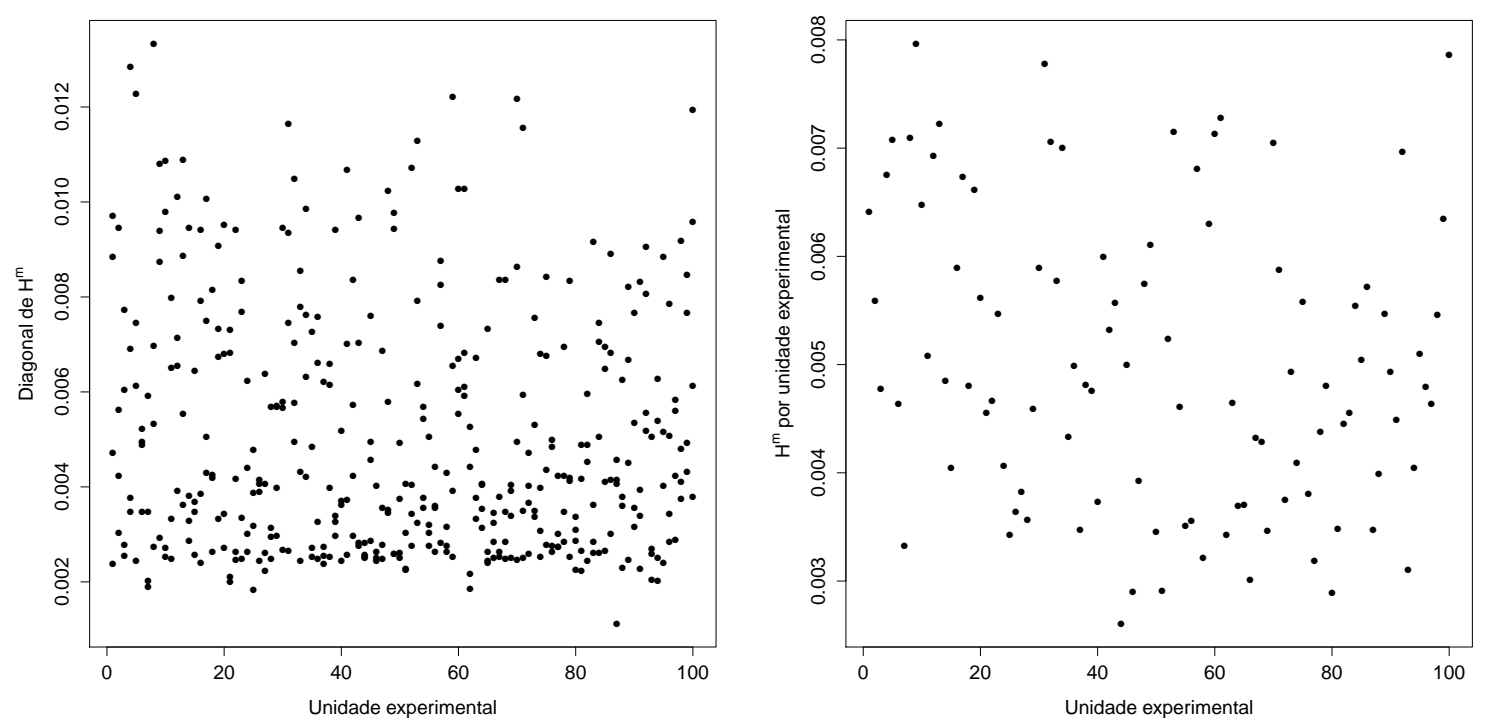

Figura 4.58: Gráficos das medidas de alavanca com base em $\boldsymbol{H}^{m}$ versus os índices das unidades experimentais do modelo com intercepto aleatório para a simulação com inclinação aleatória. 

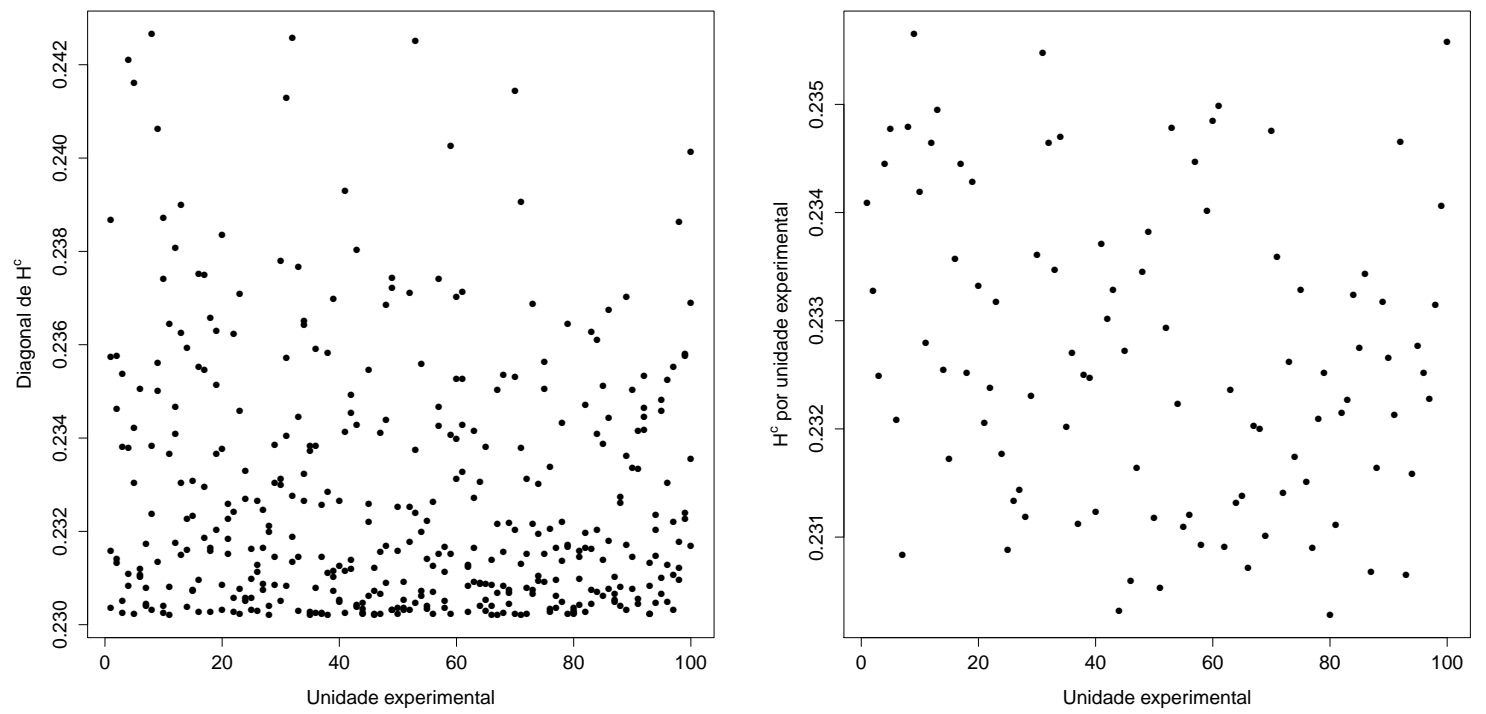

Figura 4.59: Gráficos das medidas de alavanca com base em $\boldsymbol{H}^{c}$ versus os índices das unidades experimentais do modelo com intercepto aleatório para a simulação com inclinação aleatória.

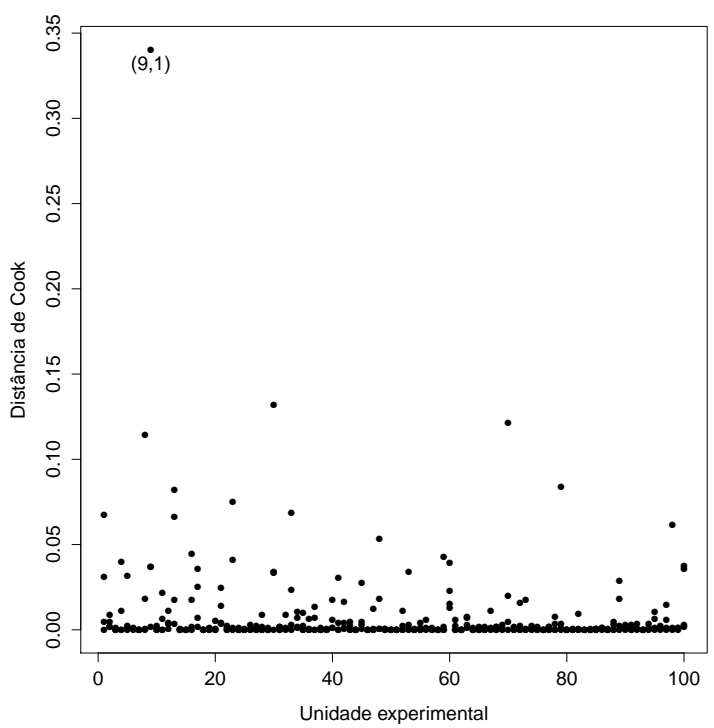

Figura 4.60: Gráfico das distâncias de Cook versus os índices das unidades experimentais do modelo com intercepto aleatório para a simulação com inclinação aleatória. 

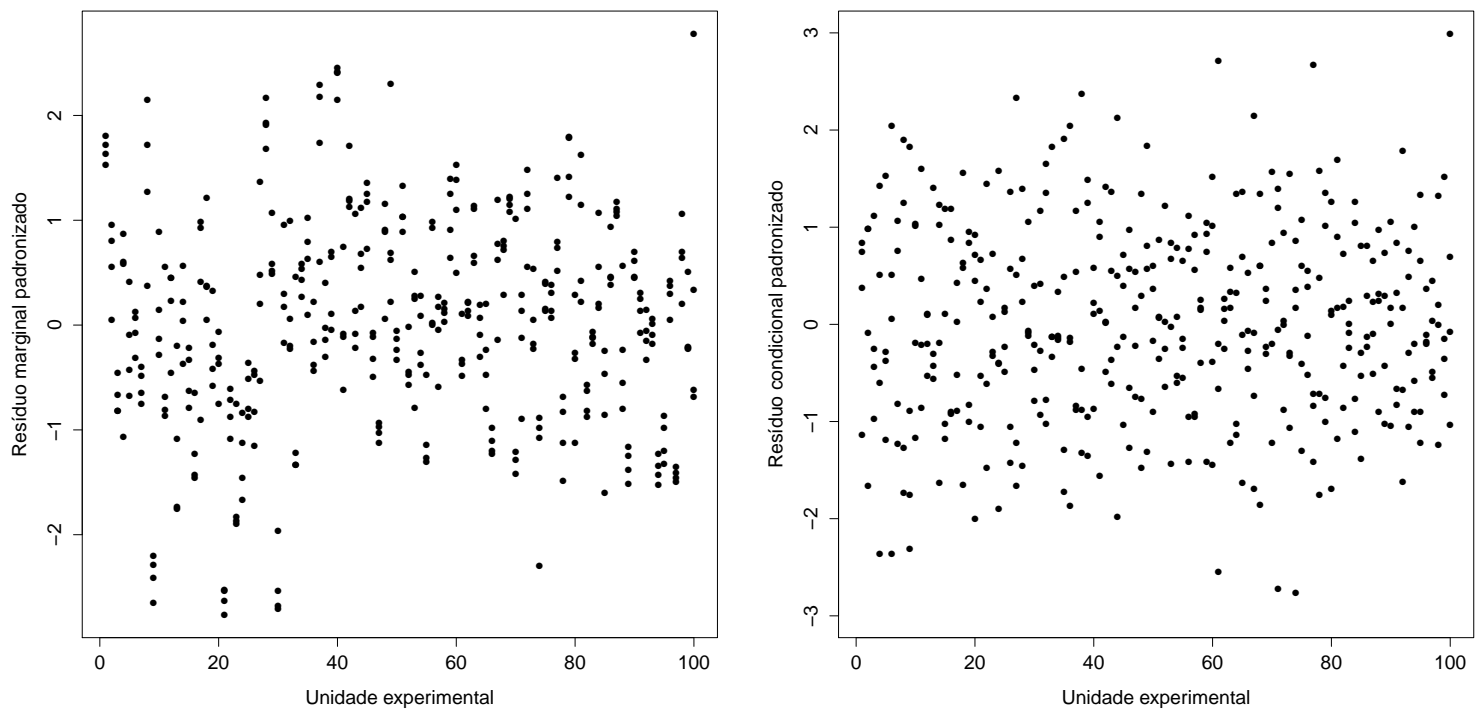

Figura 4.61: Resíduos marginais e condicionais padronizados versus os índices das unidades experimentais do modelo com intercepto e inclinação aleatórios.
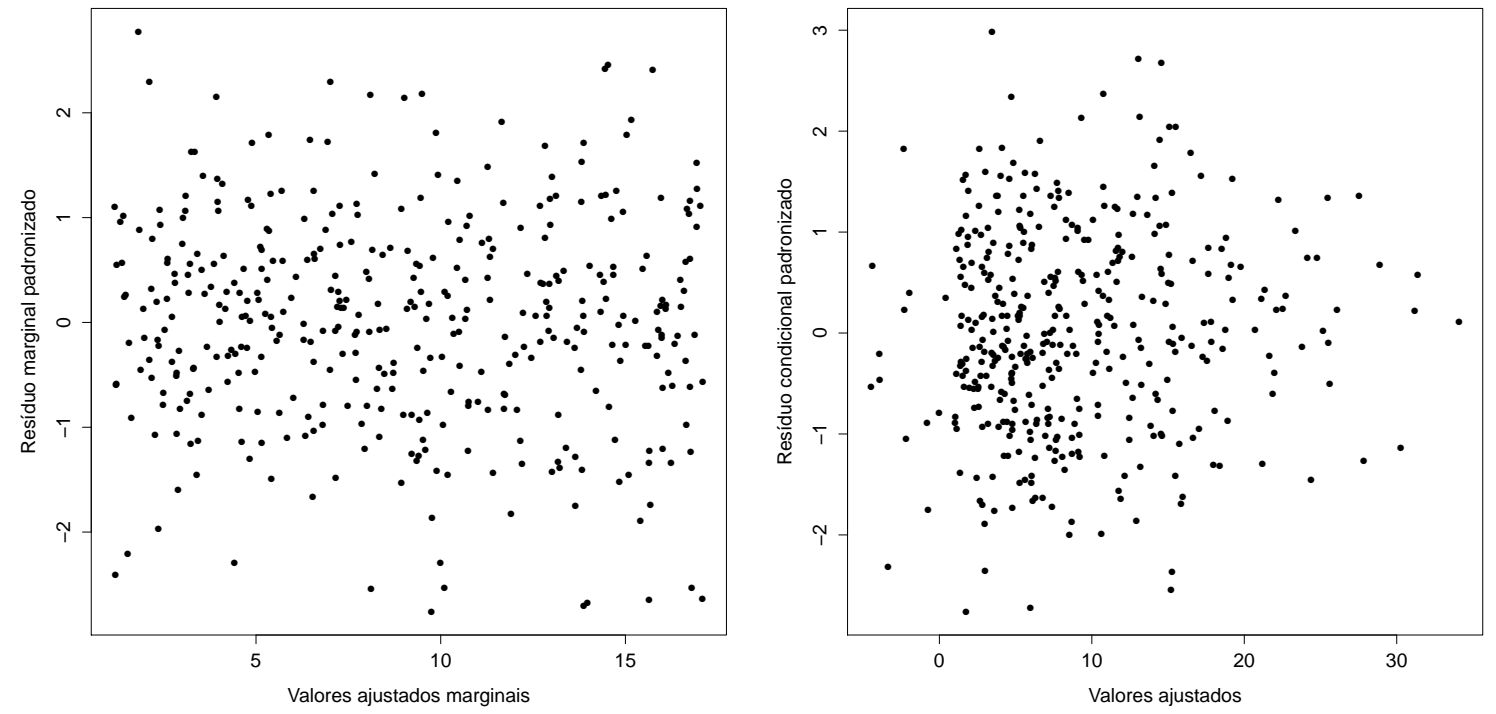

Figura 4.62: Resíduos marginais e condicionais padronizados versus os valores ajustados do modelo com intercepto e inclinação aleatórios. 


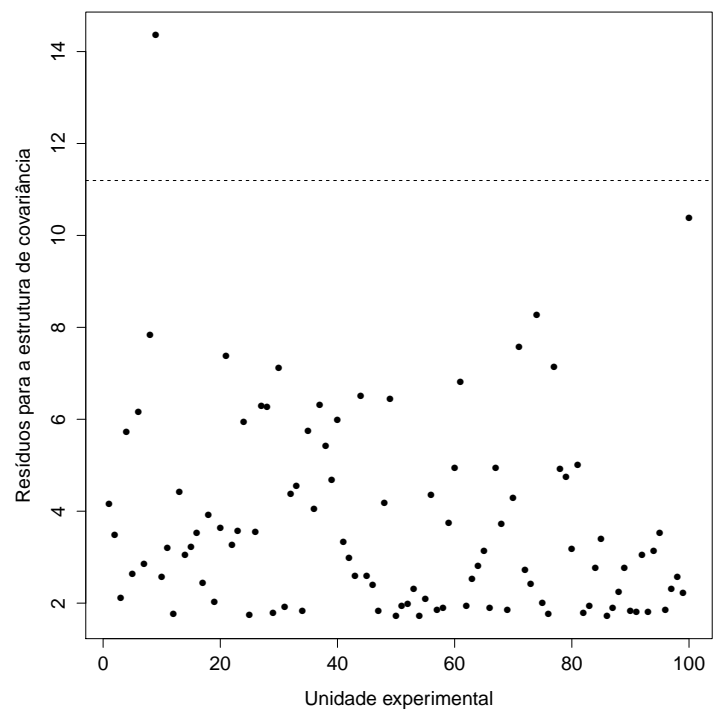

Figura 4.63: Gráfico $Q-Q$ dos resíduos para a estrutura de covariância do modelo com intercepto $e$ inclinação aleatórios.
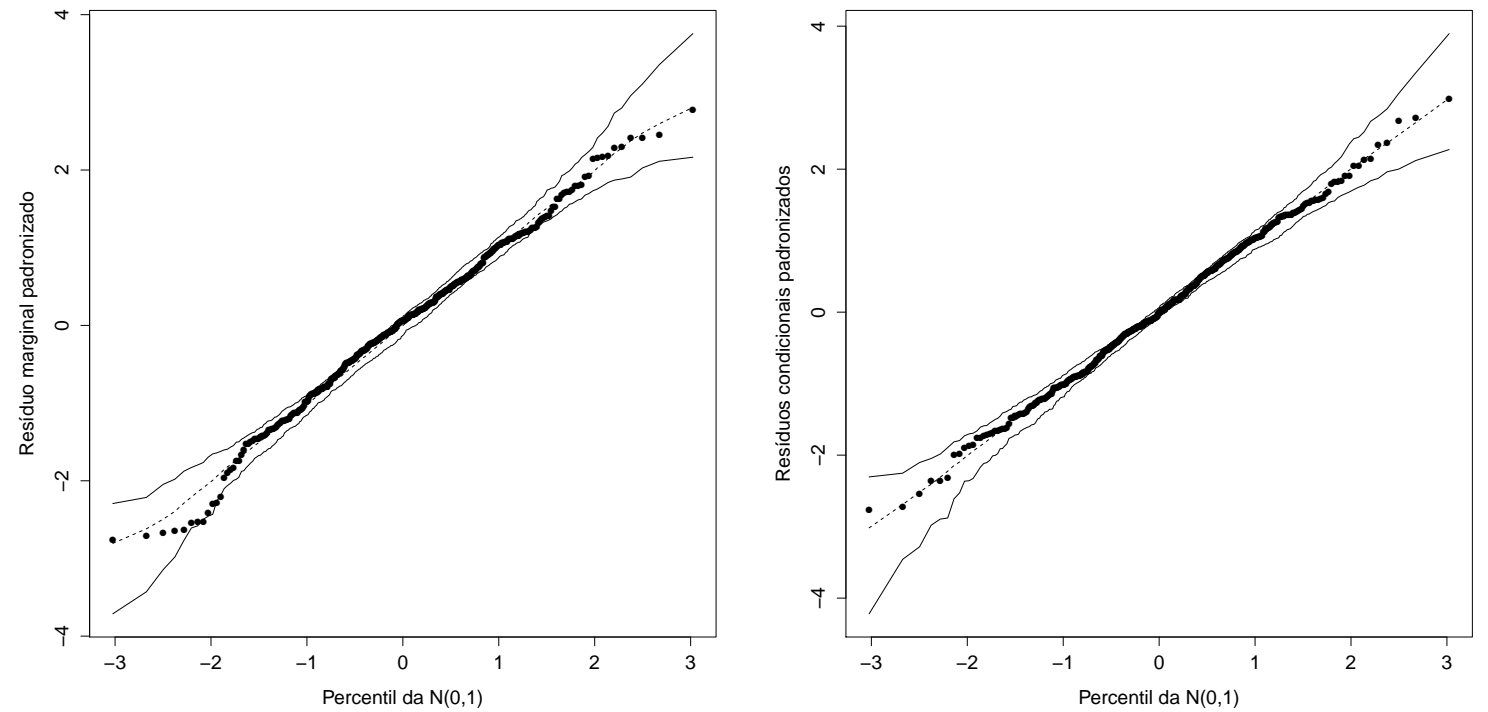

Figura 4.64: Gráficos $Q-Q$ dos resíduos marginais e condicionais padronizados do modelo com intercepto e inclinação aleatórios. 


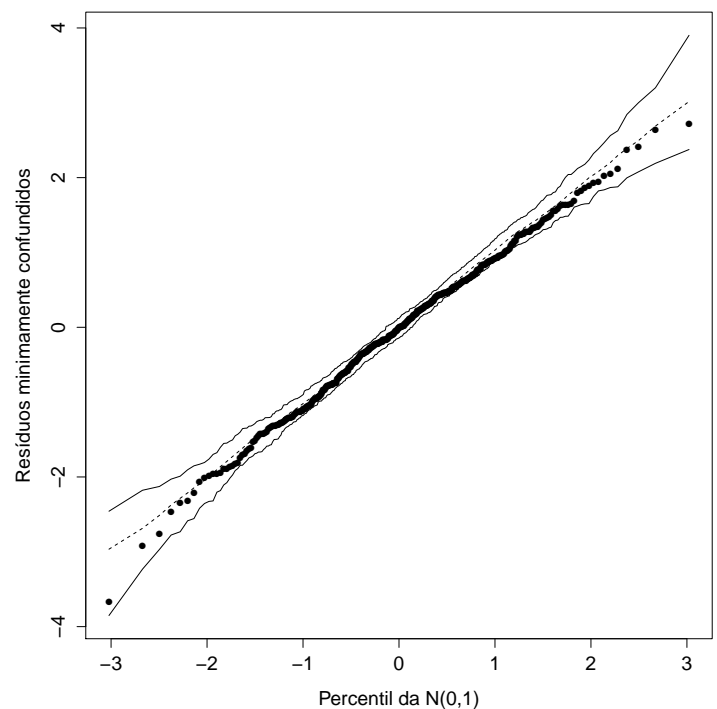

Figura 4.65: Gráfico $Q-Q$ dos resíduos minimamente confundidos do modelo com intercepto e inclinação aleatórios.

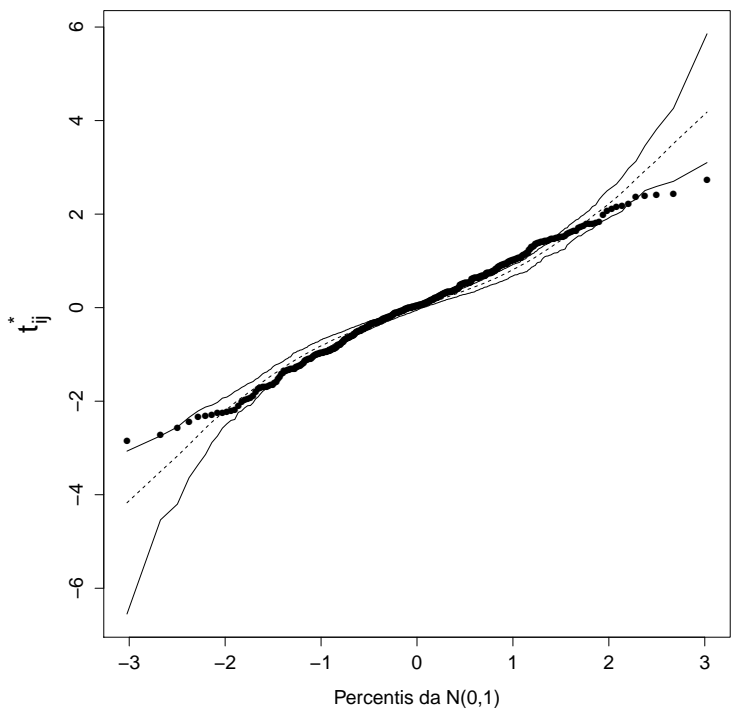

Figura 4.66: Gráfico dos resíduos $t_{i j}^{*}$ do modelo com intercepto e inclinação aleatórios. 


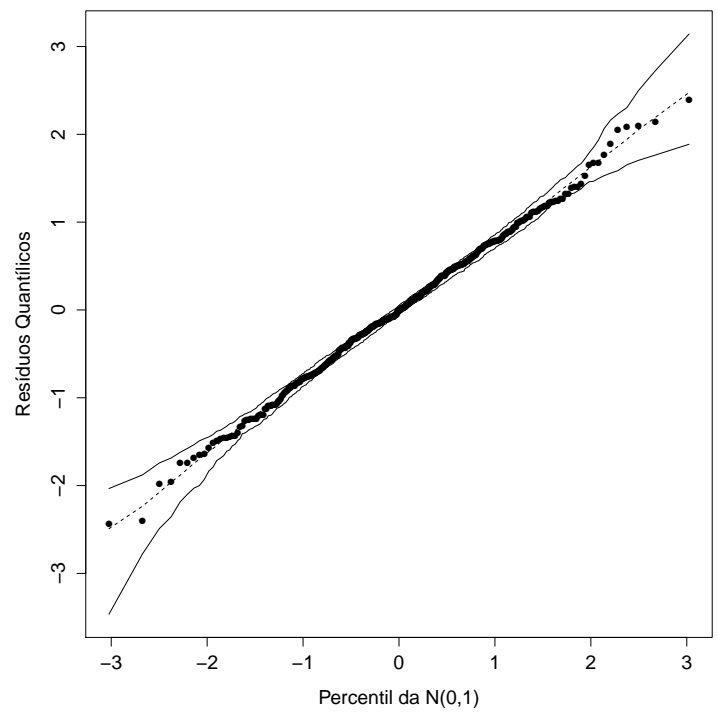

Figura 4.67: Gráfico dos resíduos quantílicos do modelo com intercepto e inclinação aleatórios.
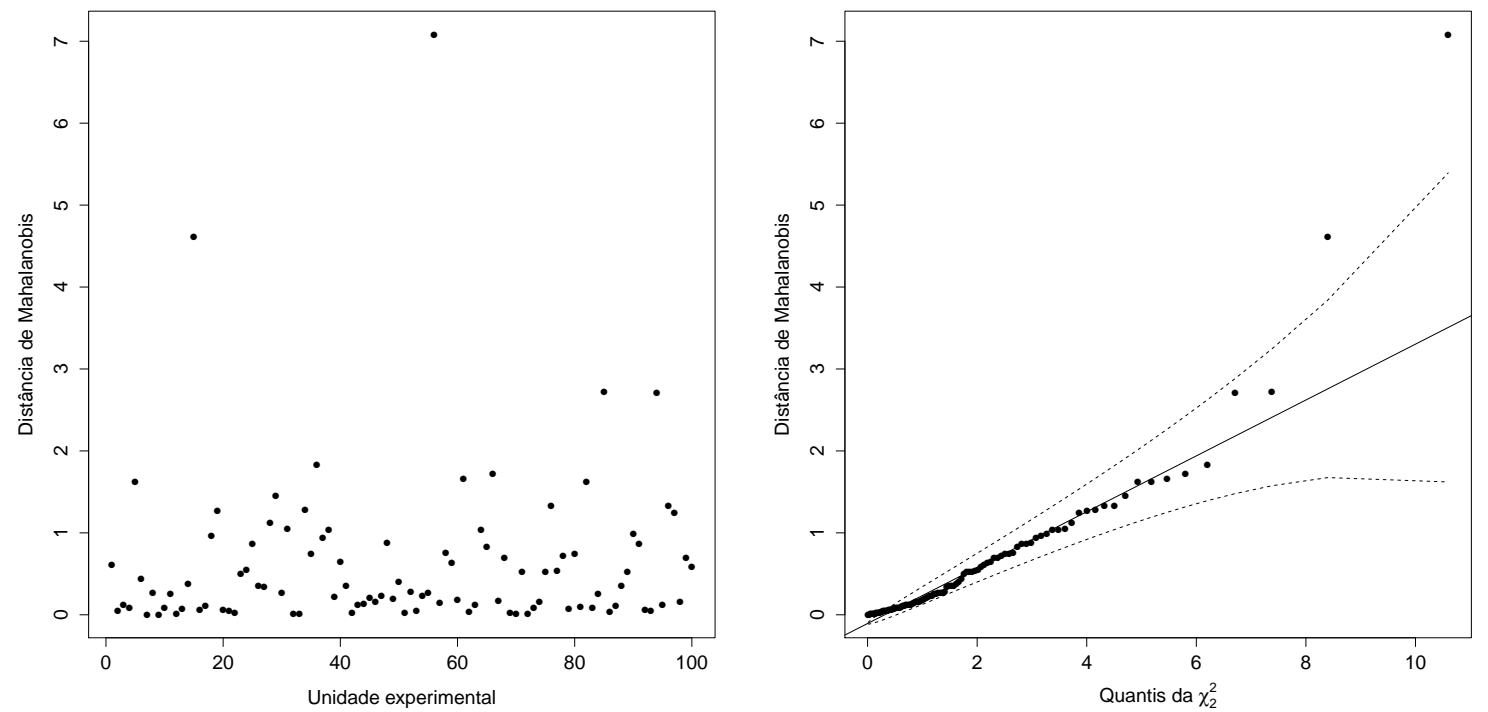

Figura 4.68: Gráficos das distâncias de Mahalanobis do modelo com intercepto e inclinação aleatórios. 

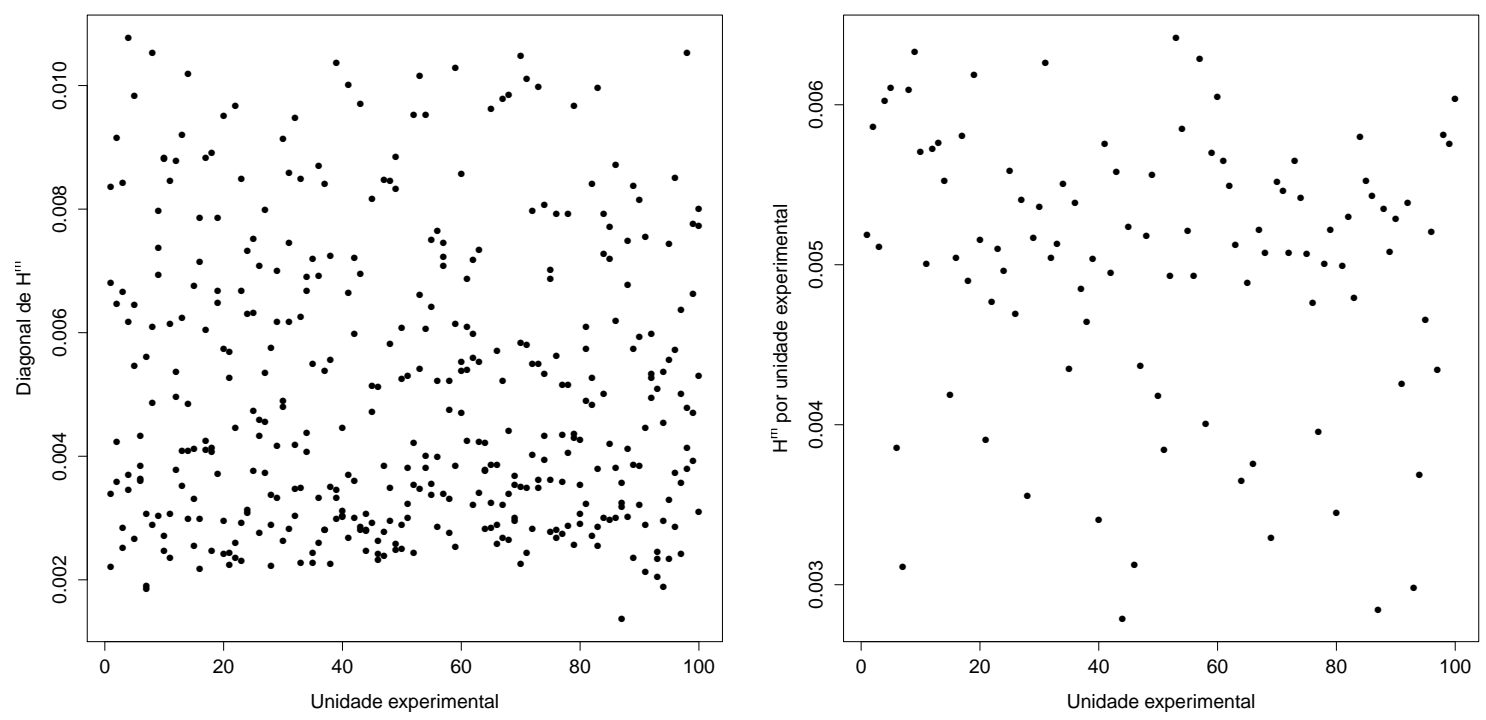

Figura 4.69: Gráficos das medidas de alavanca com base em $\boldsymbol{H}^{m}$ versus os índices das unidades experimentais do modelo com intercepto e inclinação aleatórios.
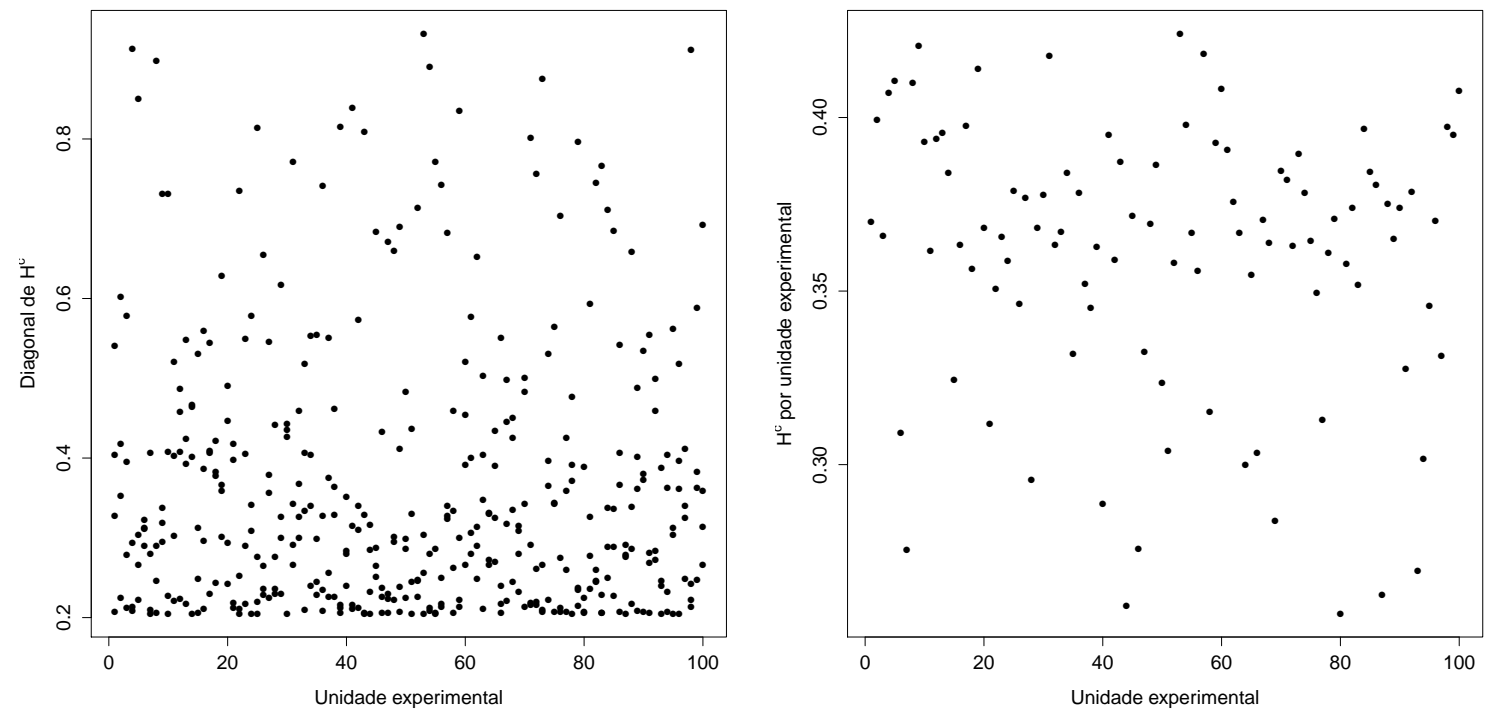

Figura 4.70: Gráficos das medidas de alavanca com base em $\boldsymbol{H}^{c}$ versus os índices das unidades experimentais do modelo com intercepto e inclinação aleatórios. 


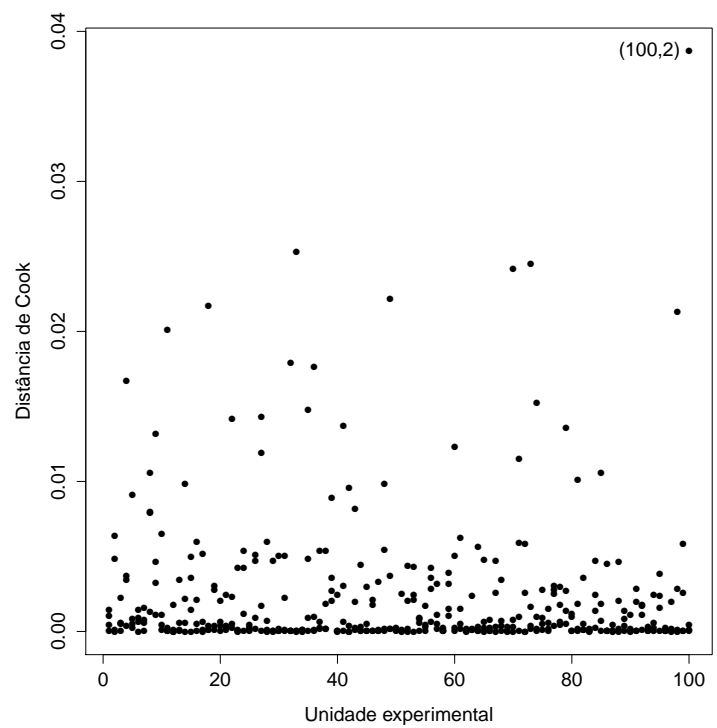

Figura 4.71: Gráfico das distâncias de Cook versus os índices das unidades experimentais do modelo com intercepto e inclinação aleatórios.
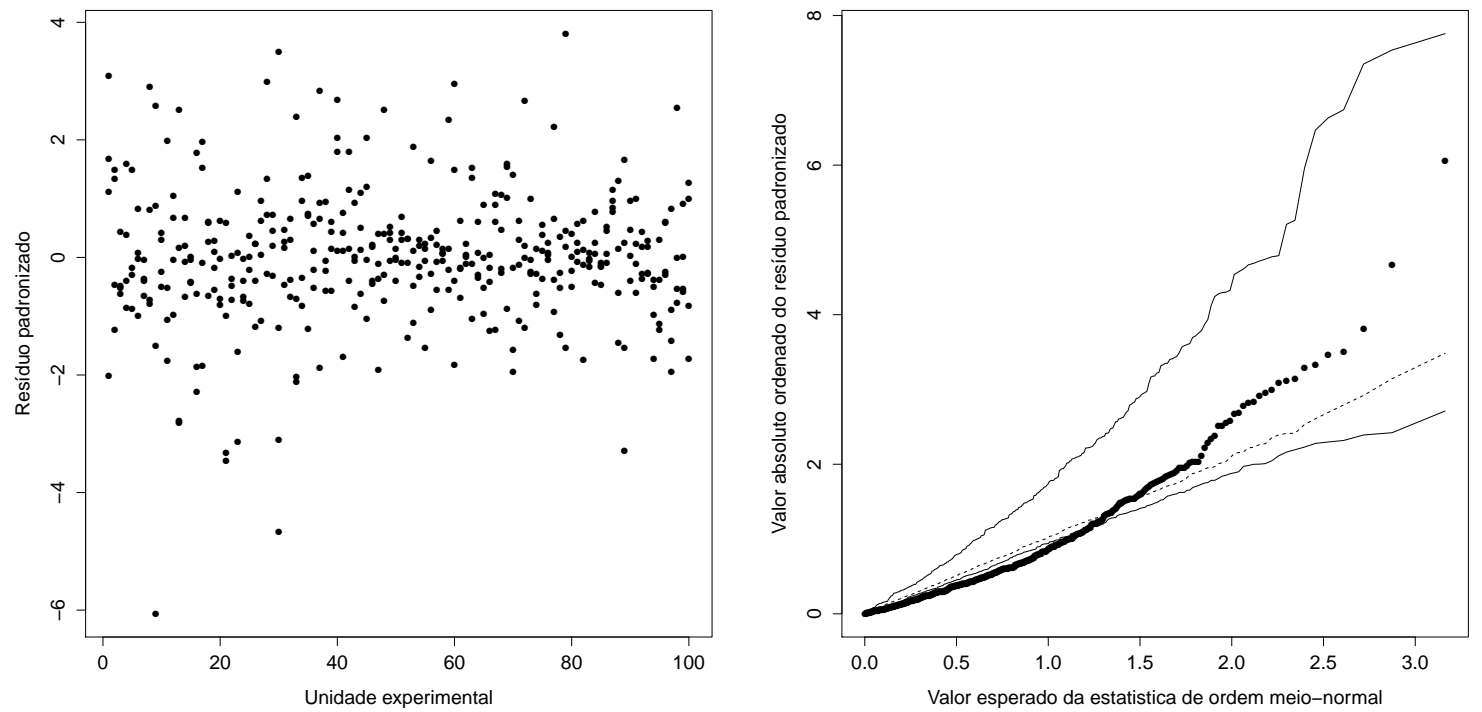

Figura 4.72: Gráficos dos resíduos do modelo marginal normal com matriz de correlações não estruturada para a simulação com intercepto e inclinação aleatórios. 

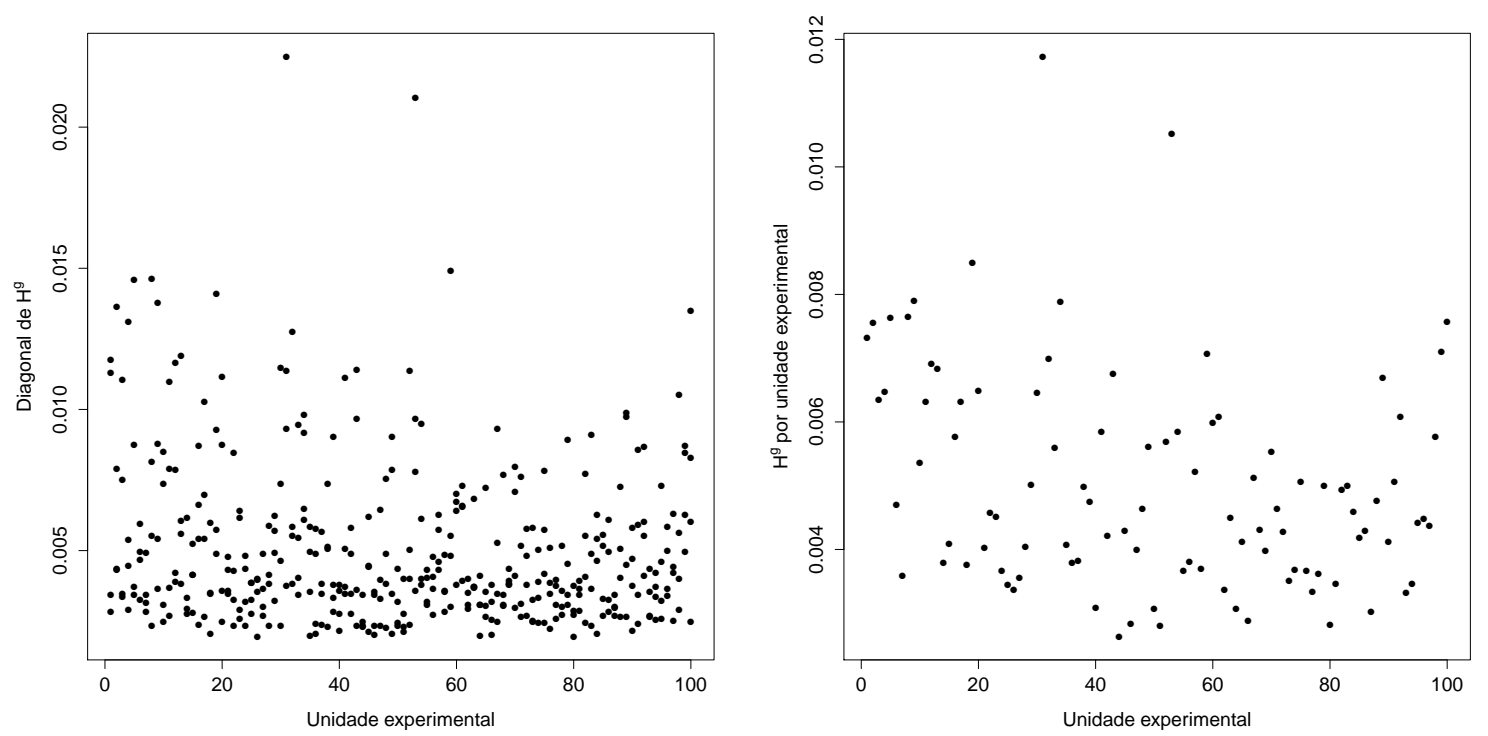

Figura 4.73: Gráficos das medidas de alavanca com base em $\boldsymbol{H}^{g}$ versus os índices das unidades experimentais do modelo marginal normal com matriz de correlações não estruturada para a simulação com intercepto e inclinação aleatórios.

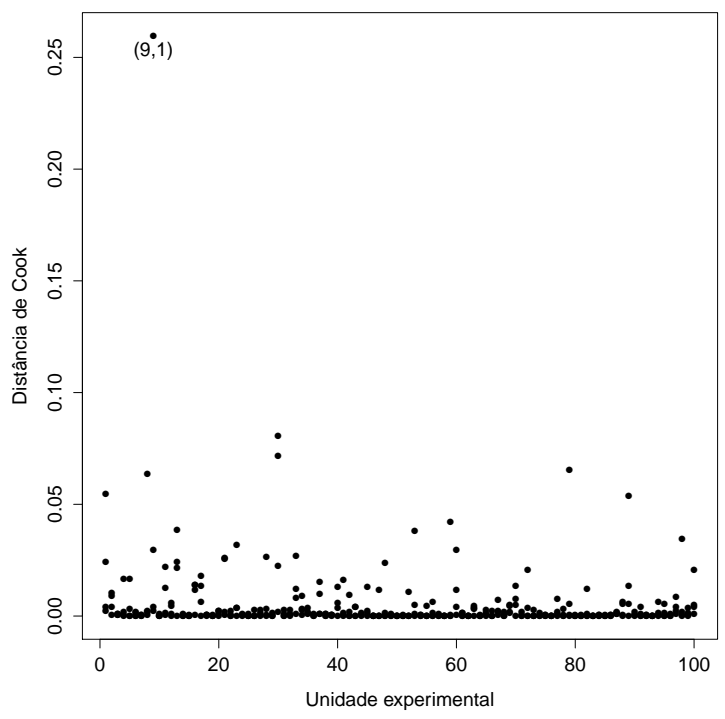

Figura 4.74: Gráfico das distâncias de Cook versus os índices das unidades experimentais do modelo marginal normal com matriz de correlações não estruturada para a simulação com intercepto e inclinação aleatórios. 


\subsection{Dados sem independência condicional}

Para avaliar o impacto de uma estrutura de covariância equivocada, consideramos agora dados obtidos a partir da simulação de um modelo sem a independência condicional, ou seja, com as matrizes $\boldsymbol{\Sigma}_{i}$ assumindo estruturas diferentes de $\sigma^{2} \boldsymbol{I}_{n_{i}}$. No exemplo, serão geradas observações baseadas no modelo

$$
\boldsymbol{y}_{i}=1+2 \boldsymbol{x}_{i}+u_{i}+\boldsymbol{\varepsilon}_{i}
$$

com $u_{i} \sim N(0,1)$ e $\varepsilon_{i} \sim N\left(\mathbf{0}, \boldsymbol{\Sigma}_{i}\right)$. Consideramos $n_{i}=8$ e a estrutura auto-regressiva de ordem 1 (AR-1) para $\boldsymbol{\Sigma}_{i}, i=1, \ldots, n$, com $\alpha=0,8$, ou seja,

$$
\boldsymbol{\Sigma}_{i}=\left[\begin{array}{cccccccc}
1 & 0,8 & 0,64 & 0,512 & 0,41 & 0,328 & 0,262 & 0,21 \\
0,8 & 1 & 0,8 & 0,64 & 0,512 & 0,41 & 0,328 & 0,262 \\
0,64 & 0,8 & 1 & 0,8 & 0,64 & 0,512 & 0,41 & 0,328 \\
0,512 & 0,64 & 0,8 & 1 & 0,8 & 0,64 & 0,512 & 0,41 \\
0,41 & 0,512 & 0,64 & 0,8 & 1 & 0,8 & 0,64 & 0,512 \\
0,328 & 0,41 & 0,512 & 0,64 & 0,8 & 1 & 0,8 & 0,64 \\
0,262 & 0,328 & 0,41 & 0,512 & 0,64 & 0,8 & 1 & 0,8 \\
0,21 & 0,262 & 0,328 & 0,41 & 0,512 & 0,64 & 0,8 & 1
\end{array}\right]
$$

Consequentemente, para cada unidade experimental, temos a matriz de covariâncias

$$
\boldsymbol{V}_{i}=\left[\begin{array}{cccccccc}
2 & 1,8 & 1,64 & 1,512 & 1,41 & 1,328 & 1,262 & 1,21 \\
1,8 & 2 & 1,8 & 1,64 & 1,512 & 1,41 & 1,328 & 1,262 \\
1,64 & 1,8 & 2 & 1,8 & 1,64 & 1,512 & 1,41 & 1,328 \\
1,512 & 1,64 & 1,8 & 2 & 1,8 & 1,64 & 1,512 & 1,41 \\
1,41 & 1,512 & 1,64 & 1,8 & 2 & 1,8 & 1,64 & 1,512 \\
1,328 & 1,41 & 1,512 & 1,64 & 1,8 & 2 & 1,8 & 1,64 \\
1,262 & 1,328 & 1,41 & 1,512 & 1,64 & 1,8 & 2 & 1,8 \\
1,21 & 1,262 & 1,328 & 1,41 & 1,512 & 1,64 & 1,8 & 2
\end{array}\right]
$$


com matriz de correlações dada por

$$
\boldsymbol{R}_{i}=\left[\begin{array}{cccccccc}
1 & 0,9 & 0,82 & 0,756 & 0,705 & 0,664 & 0,631 & 0,605 \\
0,9 & 1 & 0,9 & 0,82 & 0,756 & 0,705 & 0,664 & 0,631 \\
0,82 & 0,9 & 1 & 0,9 & 0,82 & 0,756 & 0,705 & 0,664 \\
0,756 & 0,82 & 0,9 & 1 & 0,9 & 0,82 & 0,756 & 0,705 \\
0,705 & 0,756 & 0,82 & 0,9 & 1 & 0,9 & 0,82 & 0,756 \\
0,664 & 0,705 & 0,756 & 0,82 & 0,9 & 1 & 0,9 & 0,82 \\
0,631 & 0,664 & 0,705 & 0,756 & 0,82 & 0,9 & 1 & 0,9 \\
0,605 & 0,631 & 0,664 & 0,705 & 0,756 & 0,82 & 0,9 & 1
\end{array}\right]
$$

Ajustando um modelo linear misto com intercepto aleatório, são obtidas boas estimativas dos parâmetros $\beta_{1}$ e $\beta_{2}$ (Tabela 4.12).

\begin{tabular}{crr}
\hline Parâmetro & Estimativa & Erro padrão \\
\hline$\beta_{1}$ & 1,079 & 0,129 \\
$\beta_{2}$ & 2,006 & 0,011 \\
$\sigma_{u}^{2}$ & 1,433 & \\
$\sigma^{2}$ & 0,456 & \\
\hline
\end{tabular}

Tabela 4.12: Estimativas (erros padrões) dos parâmetros do modelo linear misto na simulação com estrutura AR-1.

A partir do modelo ajustado, a matriz de correlações estimada é

$$
\hat{\boldsymbol{R}}_{i}=\left[\begin{array}{cccccccc}
1 & 0,759 & 0,759 & 0,759 & 0,759 & 0,759 & 0,759 & 0,759 \\
0,759 & 1 & 0,759 & 0,759 & 0,759 & 0,759 & 0,759 & 0,759 \\
0,759 & 0,759 & 1 & 0,759 & 0,759 & 0,759 & 0,759 & 0,759 \\
0,759 & 0,759 & 0,759 & 1 & 0,759 & 0,759 & 0,759 & 0,759 \\
0,759 & 0,759 & 0,759 & 0,759 & 1 & 0,759 & 0,759 & 0,759 \\
0,759 & 0,759 & 0,759 & 0,759 & 0,759 & 1 & 0,759 & 0,759 \\
0,759 & 0,759 & 0,759 & 0,759 & 0,759 & 0,759 & 1 & 0,759 \\
0,759 & 0,759 & 0,759 & 0,759 & 0,756 & 0,759 & 0,759 & 1
\end{array}\right]
$$

Os gráficos dos resíduos marginais e condicionais padronizados versus os índices das unidades experimentais não destacam nenhuma observação discrepante (Figura 4.75). As suposições de linearidade dos efeitos fixos e de homocedasticidade dos erros condicionais são mantidas (Figura 4.76). A Figura 4.77 apresenta o gráfico dos resíduos para a estrutura de covariância. Apenas uma unidade experimental é destacada, o que indica que não é simples detectar a violação da suposição de independência condicional por meio desse gráfico.

Os gráficos Q-Q dos resíduos marginais e condicionais padronizados do modelo indicam um 
bom ajuste (Figura 4.78). O mesmo vale para os resíduos minimamente confundidos, para os resíduos $t_{i j}^{*}$ e para os resíduos quantílicos (Figuras 4.79 a 4.81). Os gráficos das distâncias de Mahalanobis também reforçam a ideia de que o modelo está bem ajustado (Figura 4.82).

$\mathrm{O}$ ajuste de um modelo marginal normal com estrutura AR-1 resulta em boas estimativas de $\beta_{1}$ e $\beta_{2}$ (Tabela 4.13).

\begin{tabular}{crrr}
\hline Parâmetro & Estimativa & EP naive & EP robusto \\
\hline$\beta_{1}$ & 1,135 & 0,120 & 0,132 \\
$\beta_{2}$ & 1,992 & 0,008 & 0,008 \\
$\phi^{-1}$ & 1,882 & & \\
$\alpha$ & 0,892 & & \\
\hline
\end{tabular}

Tabela 4.13: Estimativas (erros padrões) dos parâmetros do modelo marginal normal com estrutura $A R-1$ na simulação com estrutura $A R-1$.

A matriz de correlações estimada do modelo é dada por

$$
\hat{\boldsymbol{R}}_{i}=\left[\begin{array}{cccccccc}
1 & 0,892 & 0,796 & 0,71 & 0,634 & 0,566 & 0,505 & 0,45 \\
0,892 & 1 & 0,892 & 0,796 & 0,71 & 0,634 & 0,566 & 0,505 \\
0,796 & 0,892 & 1 & 0,892 & 0,796 & 0,71 & 0,634 & 0,566 \\
0,71 & 0,796 & 0,892 & 1 & 0,892 & 0,796 & 0,71 & 0,634 \\
0,634 & 0,71 & 0,796 & 0,892 & 1 & 0,892 & 0,796 & 0,71 \\
0,566 & 0,634 & 0,71 & 0,796 & 0,892 & 1 & 0,892 & 0,796 \\
0,505 & 0,566 & 0,634 & 0,71 & 0,796 & 0,892 & 1 & 0,892 \\
0,45 & 0,505 & 0,566 & 0,634 & 0,71 & 0,796 & 0,892 & 1
\end{array}\right]
$$

As estimativas seguem um padrão parecido com o da estrutura original dada em (4.1). E os gráficos dos resíduos apontam para um bom ajuste do modelo (Figura 4.83).

Por fim, foi ajustado um modelo marginal normal com estrutura uniforme (Tabela 4.14).

\begin{tabular}{crrr}
\hline Parâmetro & Estimativa & EP naive & EP robusto \\
\hline$\beta_{1}$ & 1,079 & 0,129 & 0,139 \\
$\beta_{2}$ & 2,006 & 0,011 & 0,011 \\
$\phi^{-1}$ & 1,879 & & \\
\hline
\end{tabular}

Tabela 4.14: Estimativas (erros padrões) dos parâmetros do modelo marginal normal com estrutura uniforme na simulação com estrutura $A R-1$. 
A matriz de correlações estimada é

$$
\hat{\boldsymbol{R}}_{i}=\left[\begin{array}{cccccccc}
1 & 0,756 & 0,756 & 0,756 & 0,756 & 0,756 & 0,756 & 0,756 \\
0,756 & 1 & 0,756 & 0,756 & 0,756 & 0,756 & 0,756 & 0,756 \\
0,756 & 0,756 & 1 & 0,756 & 0,756 & 0,756 & 0,756 & 0,756 \\
0,756 & 0,756 & 0,756 & 1 & 0,756 & 0,756 & 0,756 & 0,756 \\
0,756 & 0,756 & 0,756 & 0,756 & 1 & 0,756 & 0,756 & 0,756 \\
0,756 & 0,756 & 0,756 & 0,756 & 0,756 & 1 & 0,756 & 0,756 \\
0,756 & 0,756 & 0,756 & 0,756 & 0,756 & 0,756 & 1 & 0,756 \\
0,756 & 0,756 & 0,756 & 0,756 & 0,756 & 0,756 & 0,756 & 1
\end{array}\right] .
$$

Os gráficos dos resíduos também indicam um bom ajuste do modelo (Figura 4.84)
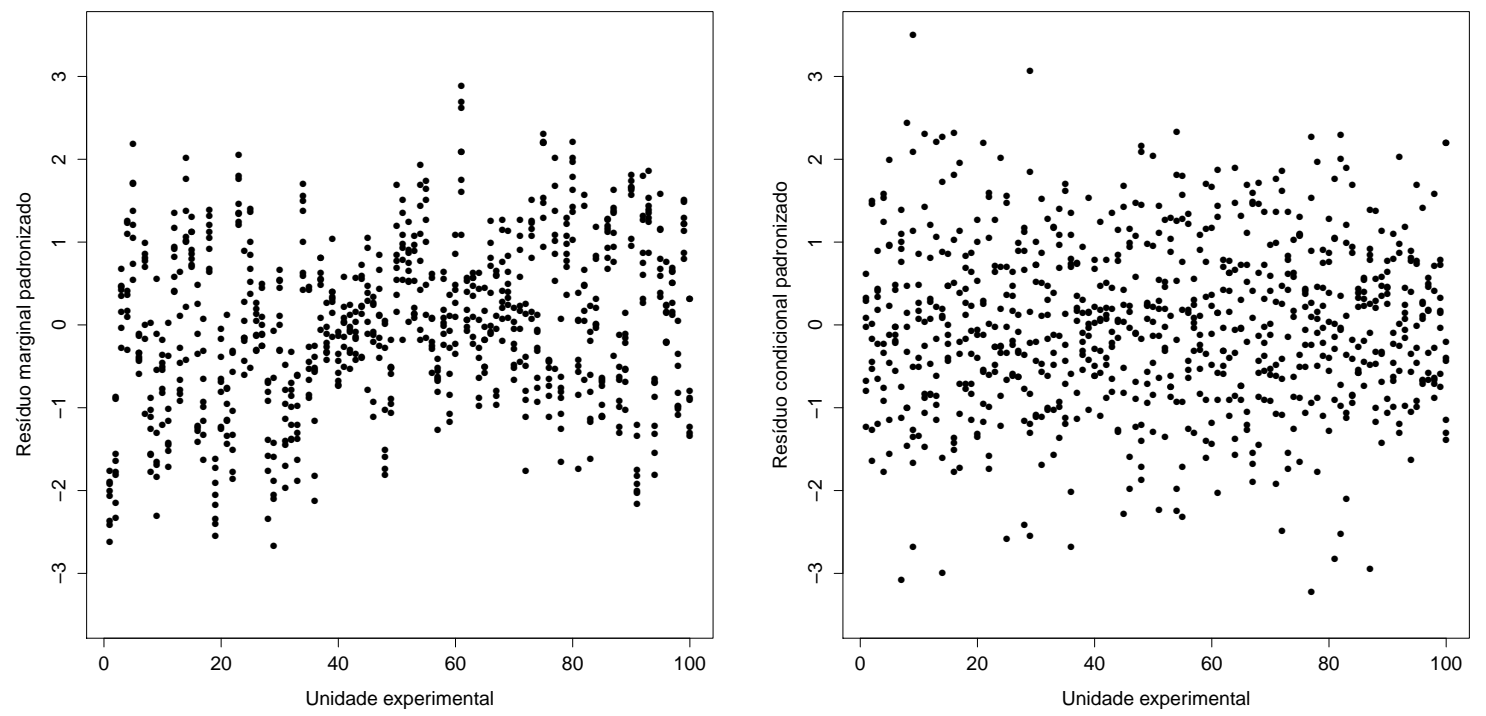

Figura 4.75: Resíduos marginais e condicionais padronizados versus os índices das unidades experimentais do modelo linear misto com intercepto aleatório na simulação com estrutura AR-1. 

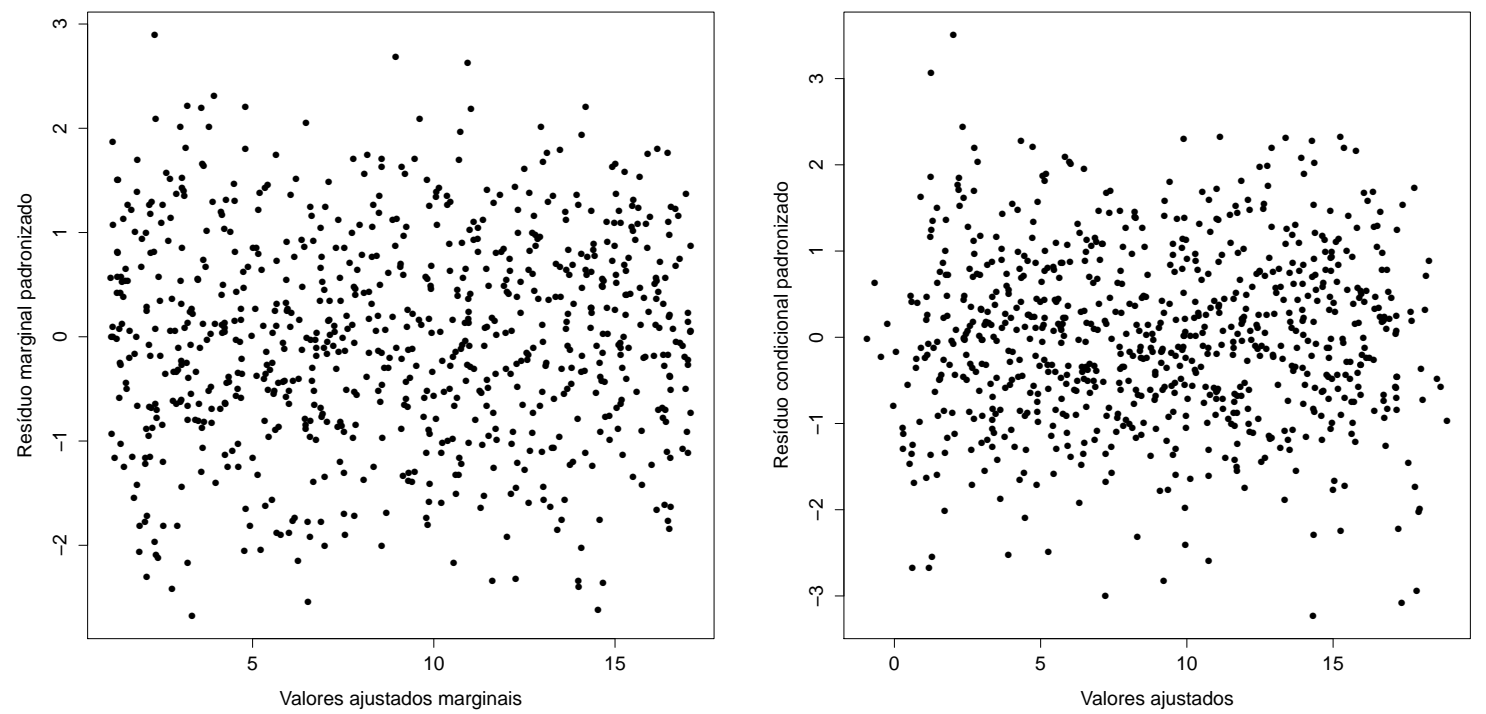

Figura 4.76: Resíduos marginais e condicionais padronizados versus os valores ajustados do modelo linear misto com intercepto aleatório na simulação com estrutura AR-1.

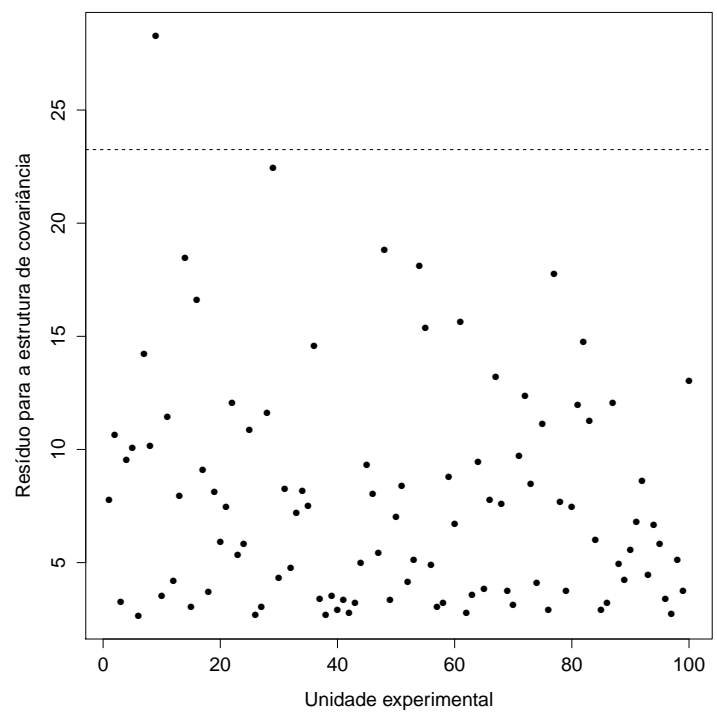

Figura 4.77: Gráfico para a estrutura de covariância do modelo linear misto com intercepto aleatório na simulação com estrutura $A R-1$. 

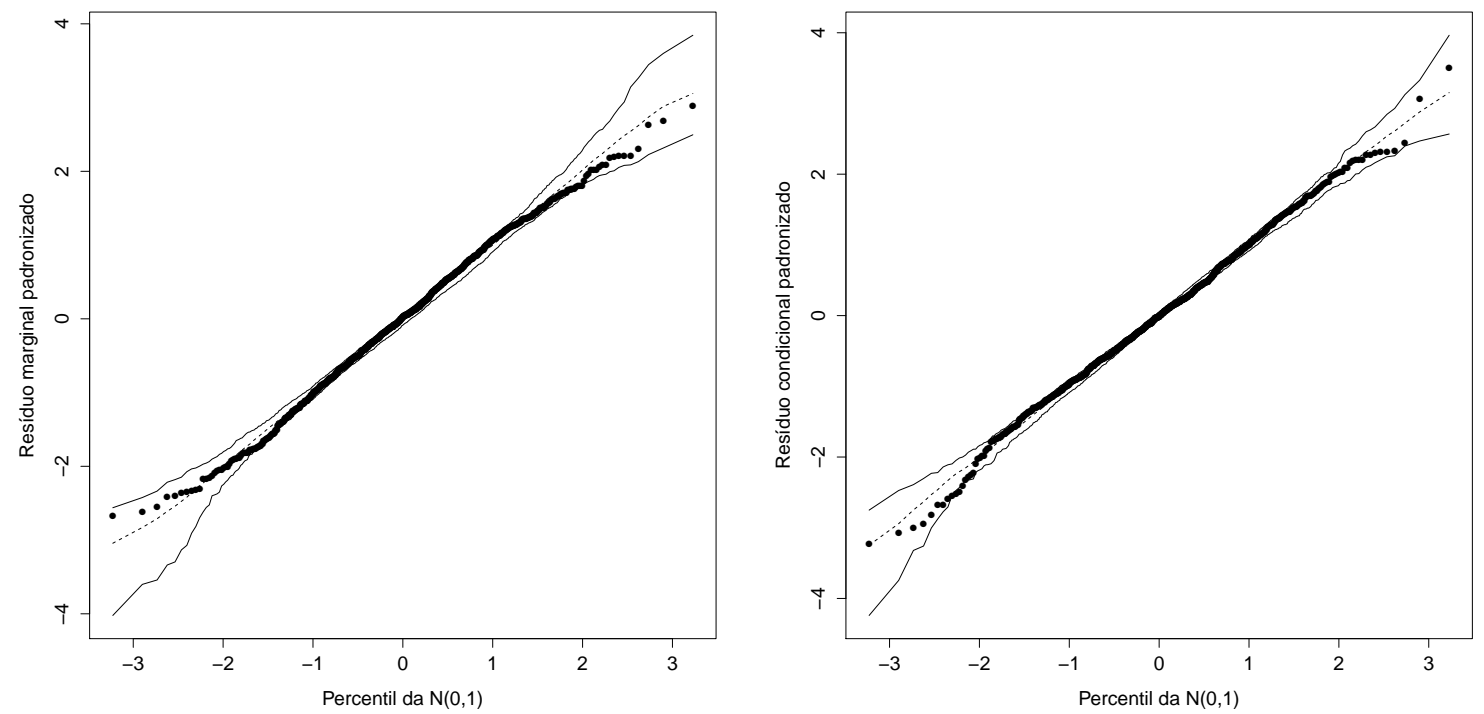

Figura 4.78: Gráficos $Q-Q$ dos resíduos marginais e condicionais padronizados do modelo linear misto com intercepto aleatório na simulação com estrutura $A R-1$.

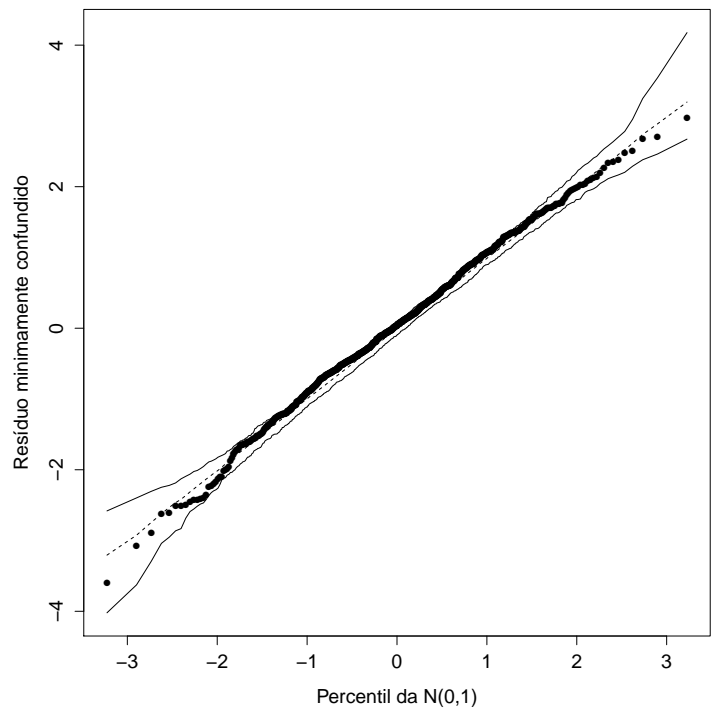

Figura 4.79: Gráfico $Q-Q$ dos resíduos minimamente confundidos do modelo linear misto com intercepto aleatório na simulação com estrutura AR-1. 


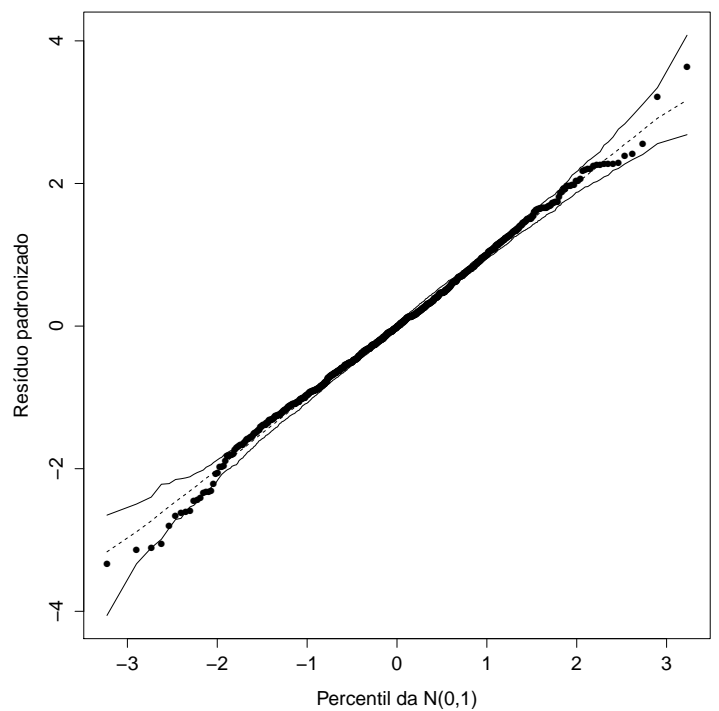

Figura 4.80: Gráfico $Q-Q$ de $t_{i j}^{*}$ do modelo linear misto com intercepto aleatório na simulação com estrutura AR-1.

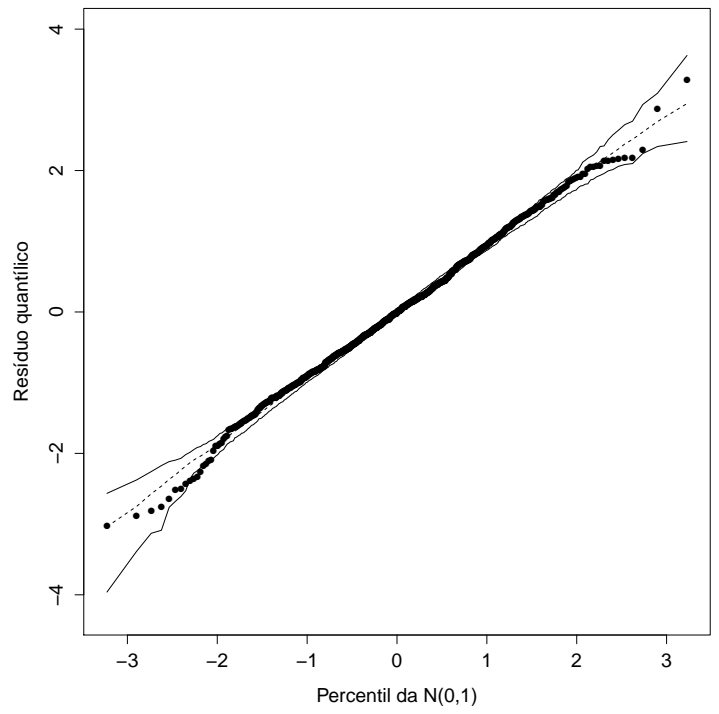

Figura 4.81: Gráfico $Q-Q$ dos resíduos quantílicos do modelo linear misto com intercepto aleatório na simulação com estrutura $A R-1$. 

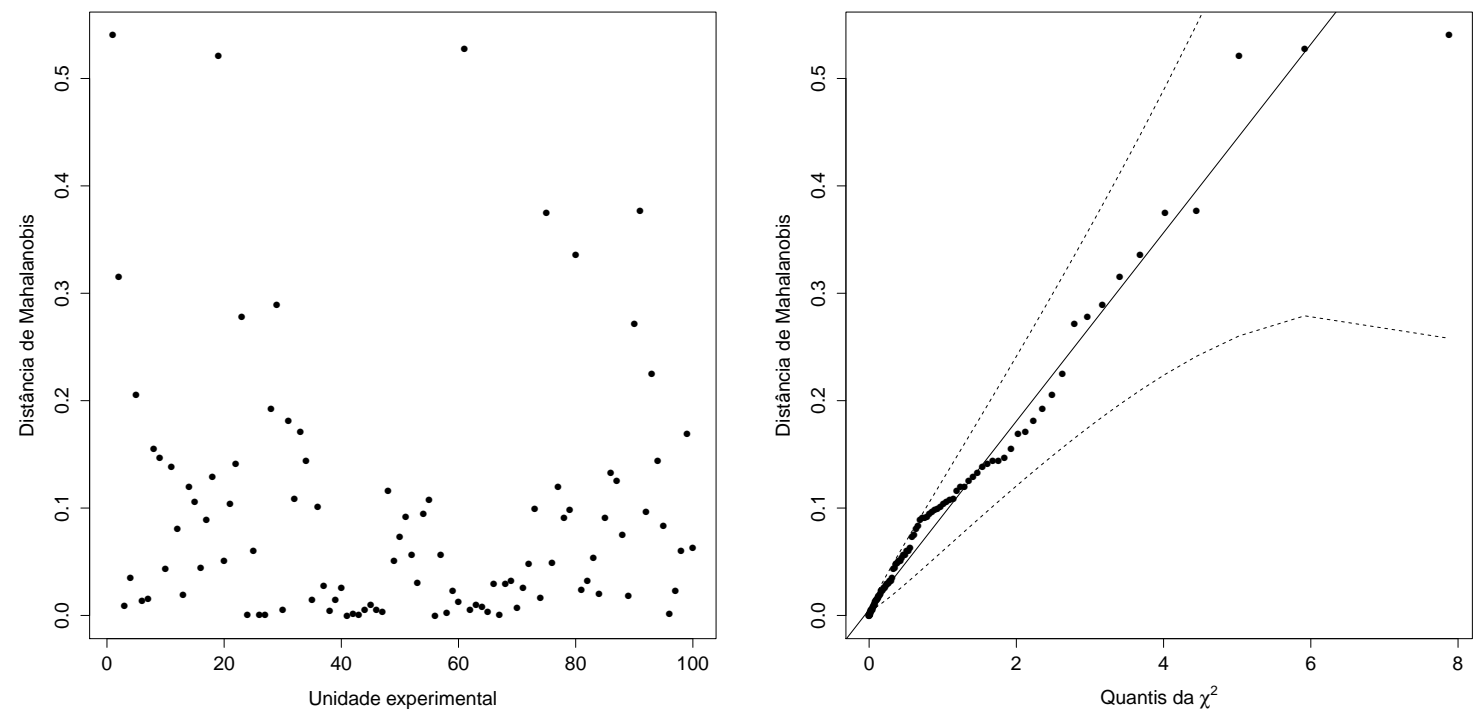

Figura 4.82: Gráficos das distâncias de Mahalanobis do modelo linear misto com intercepto aleatório na simulação com estrutura $A R-1$.
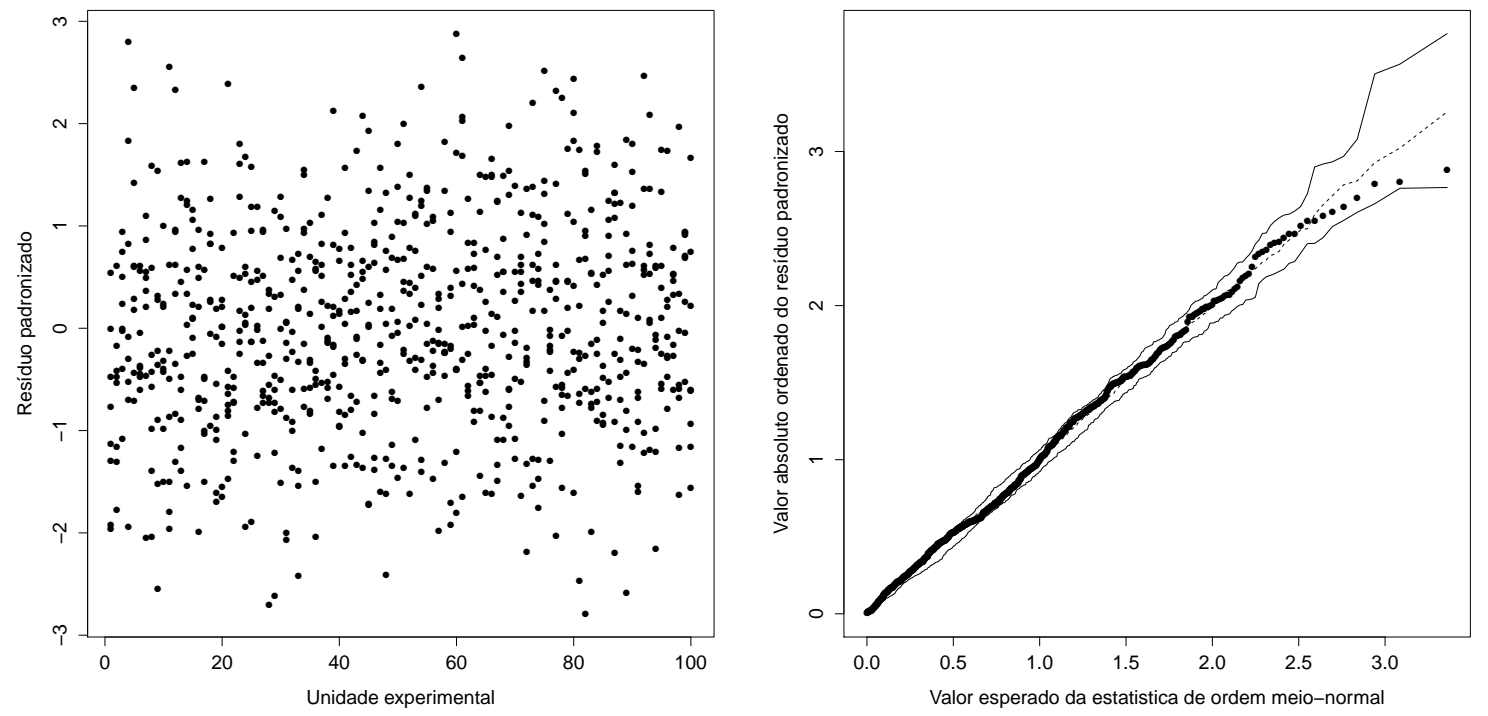

Figura 4.83: Gráficos dos resíduos do modelo marginal normal com estrutura AR-1 na simulação com estrutura AR-1. 

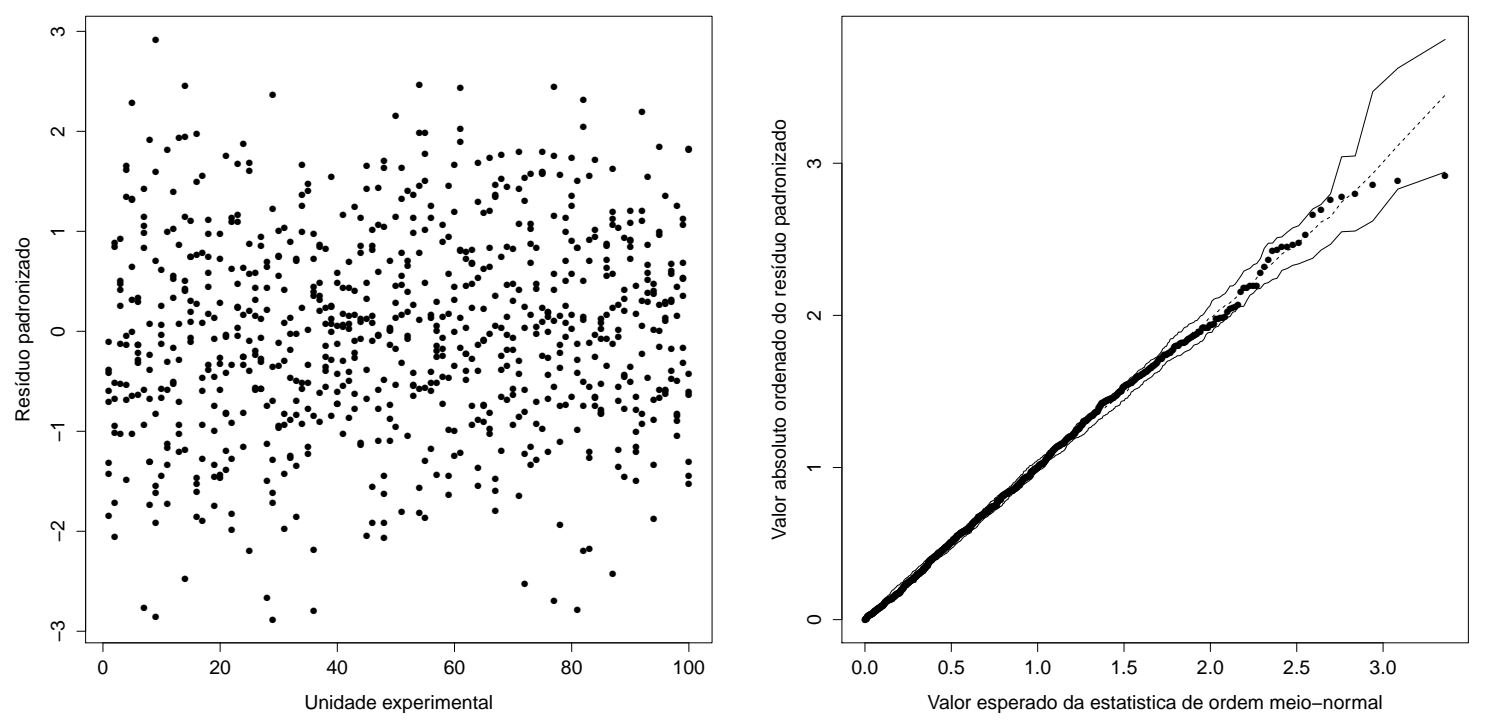

Figura 4.84: Gráficos dos resíduos do modelo marginal normal com estrutura uniforme na simulação com estrutura AR-1. 


\subsection{Recursos computacionais}

Os ajustes considerados neste trabalho foram realizados no software $\mathrm{R}$ (v2.15.0). O pacote gee foi utilizado nos ajustes dos modelos marginais. As rotinas utilizadas nas técnicas de diagnóstico desses modelos foram obtidas a partir daquelas apresentadas em Venezuela [2003].

Os modelos lineares mistos foram ajustados através do pacote 1 me 4 , que também pode ajustar modelos lineares generalizados mistos [Bates, 2012]. Para os modelos lineares generalizados hierárquicos, foi utilizado o pacote hglm, que considera o processo iterativo dado em (2.14) [Rönnegård et al., 2010]. Outra possibilidade para o ajuste desses modelos é o pacote HGLMMM, que considera o algoritmo de Newton-Raphson diretamente na função de verossimilhança- $h$ [Molas e Lesaffre, 2011]. As rotinas para as técnicas de diagnóstico dos modelos lineares mistos foram baseadas naquelas utilizadas por Nobre [2004] e Manghi [2012]. Para os modelos lineares generalizados hierárquicos, as rotinas para as técnicas de diagnóstico foram adaptadas a partir do que estava disponível nos pacotes hglm e HGLMMM. Os pacotes utilizados para os modelos com efeitos aleatórios utilizam os resíduos condicionais como padrão e, quando a distribuição é diferente da normal, são utilizados os resíduos componentes do desvio. As rotinas utilizadas no R estão apresentadas no Apêndice A. 


\section{Capítulo 5}

\section{Aplicação}

Como exemplo de aplicação da metodologia apresentada, foi selecionado um estudo que, motivado pelo crescimento da utilização de técnicas de inseminação artificial em suínos, teve como objetivo a identificação e controle dos fatores que influenciam na qualidade do sêmen em porcos [Paula et al., 2005]. O estudo envolveu 10 porcos machos, adultos, de mesma raça, linhagem e idade, estudados ao longo das quatro estações do ano com início no inverno de 2004 e término no outono de 2005.

Nesta aplicação o objetivo é avaliar o efeito da estação do ano sobre o volume do ejaculado (em $m l)$.

A Tabela 5.1 apresenta o volume do ejaculado por porco e por estação do ano.

\begin{tabular}{|c|c|c|c|c|c|}
\hline \multirow[b]{2}{*}{ Porco } & \multicolumn{4}{|c|}{ Estação } & \multirow[b]{2}{*}{ Média geral } \\
\hline & $\begin{array}{c}\text { Inverno } \\
j=1\end{array}$ & $\begin{array}{c}\text { Primavera } \\
\quad j=2\end{array}$ & $\begin{array}{l}\text { Verão } \\
j=3\end{array}$ & $\begin{array}{c}\text { Outono } \\
j=4\end{array}$ & \\
\hline 1 & 234 & 233 & 272 & 366 & 276 \\
\hline 2 & 145 & 155 & 226 & 253 & 195 \\
\hline 3 & 245 & 296 & 302 & 405 & 312 \\
\hline 4 & 297 & 329 & 336 & 380 & 335 \\
\hline 5 & 335 & 377 & 459 & 498 & 417 \\
\hline 6 & 271 & 230 & 243 & 299 & 261 \\
\hline 7 & 254 & 253 & 261 & 345 & 278 \\
\hline 8 & 268 & 296 & 350 & 439 & 339 \\
\hline 9 & 244 & 282 & 264 & 338 & 282 \\
\hline 10 & 231 & 230 & 264 & 322 & 262 \\
\hline Média geral & 252 & 268 & 298 & 364 & 296 \\
\hline
\end{tabular}

Tabela 5.1: Volume do ejaculado (em $\mathrm{ml}$ ) para os suínos.

Analisando os dados descritivamente, pode-se notar que o maior volume ocorreu no outono, enquanto o menor volume ocorreu no inverno. O porco 5 é destacado como aquele com o maior volume. O porco 2 obteve o menor volume. Além disso, todos os porcos parecem seguir o mesmo padrão, com volumes maiores no outono e menores no inverno (Figura 5.1). 


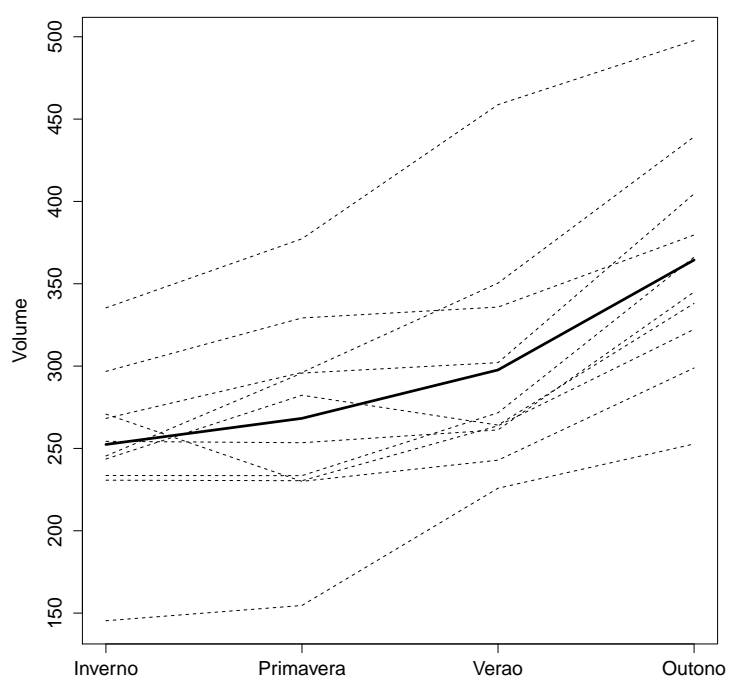

Figura 5.1: Gráfico do volume do ejaculado (em ml) para os suínos.

\section{Modelo 1}

Primeiramente será ajustado um modelo linear misto com intercepto aleatório, sendo

$$
y_{i j}=\beta_{1}+\beta_{p} x_{p j}+\beta_{v} x_{v i j}+\beta_{o} x_{o i j}+u_{i}+\varepsilon_{i j},
$$

em que $y_{i j}$ representa o volume do ejaculado da $j$-ésima medida do $i$-ésimo suíno, $u_{i} \sim N\left(0, \sigma_{u}^{2}\right)$ é o intercepto aleatório associado ao $i$-ésimo animal, $\varepsilon_{i j} \sim N\left(0, \sigma^{2}\right)$ independentes e as covariáveis relacionadas às estações do ano são definidas como

$$
\begin{aligned}
& x_{p i j}= \begin{cases}1, & \text { se } j=2 ; \\
0, & \text { caso contrário, }\end{cases} \\
& x_{v i j}= \begin{cases}1, & \text { se } j=3 ; \\
0, & \text { caso contrário, }\end{cases} \\
& x_{o i j}= \begin{cases}1, & \text { se } j=4 ; \\
0, & \text { caso contrário, }\end{cases}
\end{aligned}
$$

para $i=1, \ldots, 10$ e $j=1, \ldots, 4$. Dessa forma, $\beta_{1}$ representa o valor esperado do volume do ejaculado para uma medida realizada no inverno. Os parâmetros $\beta_{p}, \beta_{v}$ e $\beta_{o}$ representam a diferença esperada com relação ao volume do ejaculado médio de inverno, quando a medida é realizada na primavera, verão e outono, respectivamente.

As estimativas dos parâmetros obtidas no ajuste estão na Tabela 5.2.

Segundo o modelo ajustado, o volume esperado do ejaculado no inverno é de 252, $410 \mathrm{ml}$. Com relação ao volume esperado no inverno, espera-se que o volume seja 112, $010 \mathrm{ml}$ maior no outono, 45, $250 \mathrm{ml}$ maior no verão e 15,860 $\mathrm{ml}$ maior na primavera. Considerando um nível de significância de $5 \%$, a diferença da primavera em relação ao inverno não é significativa. $\mathrm{O}$ coeficiente de correlação intraclasse estimado é 0,849 . 


\begin{tabular}{crr}
\hline Parâmetro & Estimativa & Erro padrão \\
\hline$\beta_{1}$ & 252,410 & 19,990 \\
$\beta_{p}$ & 15,860 & 10,980 \\
$\beta_{v}$ & 45,250 & 10,980 \\
$\beta_{o}$ & 112,010 & 10,980 \\
$\sigma_{u}^{2}$ & 3394,700 & \\
$\sigma^{2}$ & 603,100 & \\
\hline
\end{tabular}

Tabela 5.2: Estimativas (erros padrões) dos parâmetros - modelo 1.

Os gráficos dos resíduos marginais e condicionais padronizados destacam algumas observações do porco 5 como discrepantes (Figura 5.2). A partir dos gráficos desses mesmos resíduos contra os valores ajustados, é possível ver que não há indícios de violação nas suposições de linearidade dos efeitos fixos e homocedasticidade dos erros condicionais (Figura 5.3). O gráfico para as estruturas de covariância não destaca nenhum porco fora da faixa prevista (Figura 5.4).

As Figuras 5.5 a 5.8 apresentam os gráficos do tipo Q-Q para a distribuição normal, que indicam que o modelo está bem ajustado.

Considerando os gráficos para a distância de Mahalanobis (Figura 5.9), os porcos 2 e 5 apresentaram valores altos. O porco 2 por ter volume baixo e o porco 5 por ter volume alto. Os valores discrepantes desses dois porcos também afetaram o gráfico Q-Q para a distribuição $\chi_{1}^{2}$.

A observação do porco 6 obtida no inverno obteve o maior valor de distância de Cook (Figura 5.10). Trata-se do único porco que obteve no inverno um volume médio maior do que o observado no verão e na primavera. Ajustando o modelo sem essa observação, obtemos $\hat{\beta}_{1}=224,728$, $\hat{\beta}_{p}=23,541, \hat{\beta}_{v}=52,933$ e $\hat{\beta}_{o}=119,695, \operatorname{com} \hat{\sigma}^{2}=463,240$ e $\hat{\sigma}_{u}^{2}=3620,450$. A mudança acontece na diferença da primavera com relação ao inverno, que agora passa a ser significativa ao nível de significância de $5 \%$.

\section{Modelo 2}

Na Figura 5.1 observa-se um efeito linear da estação do ano sobre o volume do ejaculado. Assim, outra possibilidade de análise é considerar a variável associada às estações do ano como uma variável numérica, com os valores 1, 2, 3 e 4 representando a estação do ano, conforme a Tabela 5.1. Logo, o modelo linear misto com intercepto aleatório é dado por

$$
y_{i j}=\beta_{1}+\beta_{2} x_{i j}+u_{i}+\varepsilon_{i j}
$$

em que $x_{i j}=j, u_{i} \sim N\left(0, \sigma_{u}^{2}\right)$ é o intercepto aleatório associado ao $i$-ésimo animal e $\varepsilon_{i j} \sim$ $N\left(0, \sigma^{2}\right)$ independentes, para $i=1, \ldots, 10$ e $j=1, \ldots, 4$.

Segundo os resultados apresentados na Tabela 5.3, o volume esperado do ejaculado no inverno é de $204,335+36,542=240,877 \mathrm{ml}$, com aumento esperado de $36,542 \mathrm{ml}$ a cada vez que passamos para a estação seguinte.

A Figura 5.11 apresenta os gráficos dos resíduos marginais e condicionais padronizados. Nos gráficos dos resíduos contra os valores ajustados (Figura 5.12), não se percebe nenhum indício de violação nas suposições de linearidade dos efeitos fixos e de homocedasticidade dos erros 


\begin{tabular}{crr}
\hline Parâmetro & Estimativa & Erro padrão \\
\hline$\beta_{1}$ & 204,335 & 21,304 \\
$\beta_{2}$ & 36,542 & 3,987 \\
$\sigma_{u}^{2}$ & 3346,82 & \\
$\sigma^{2}$ & 794,69 & \\
\hline
\end{tabular}

Tabela 5.3: Estimativas (erros padrões) dos parâmetros - modelo 2.

condicionais. Nenhum porco apresenta valor fora da faixa esperada para os resíduos da estrutura de covariância (Figura 5.13). As Figuras 5.14 a 5.17 apresentam os gráficos Q-Q do modelo ajustado. Essas figuras indicam um bom ajuste do modelo, com exceção do gráfico dos resíduos minimamente confundidos (Figura 5.15), que apresenta alguns pontos fora da região esperada. Mais uma vez os porcos 2 e 5 são destacados como unidades experimentais discrepantes pelo gráfico das distâncias de Mahalanobis, o que interfere também no gráfico Q-Q da distribuição $\chi_{1}^{2}$ para esses valores (Figura 5.18).

No gráfico das distâncias de Cook (Figura 5.19), a observação 1 do porco 6 é destacada como influente. Ajustando o modelo sem essa observação, são obtidas as estimativas $\hat{\beta}_{1}=195,427$ e $\hat{\beta}_{2}=39,215, \operatorname{com} \hat{\sigma}^{2}=608,860$ e $\hat{\sigma}_{u}^{2}=3621,660$. Apesar da variação com relação às estimativas obtidas no conjunto de dados completo, isso não representa mudança inferencial no ajuste do modelo.

\section{Modelo 3}

Como no modelo anterior nem todos os gráficos de diagnóstico indicaram um bom ajuste, consideramos um modelo com intercepto e inclinação aleatórios dado por

$$
y_{i j}=\beta_{1}+\beta_{2} x_{i j}+u_{1 i}+u_{2 i} x_{i j}+\varepsilon_{i j}
$$

em que $x_{i j}=j, u_{1 i} \sim N\left(0, \sigma_{u 1}^{2}\right)$ é o intercepto aleatório, $u_{2 i} \sim N\left(0, \sigma_{u 2}^{2}\right)$ é a inclinação aleatória e $\varepsilon_{i j} \sim N\left(0, \sigma^{2}\right)$ independentes, para $i=1, \ldots, 10$ e $j=1, \ldots, 4$.

\begin{tabular}{crr}
\hline Parâmetro & Estimativa & Erro padrão \\
\hline$\beta_{1}$ & 204,335 & 17,564 \\
$\beta_{2}$ & 36,542 & 5,113 \\
$\sigma_{u 1}^{2}$ & 2181,2 & \\
$\sigma_{u 2}^{2}$ & 140,98 & \\
$\sigma^{2}$ & 602,58 & \\
\hline
\end{tabular}

Tabela 5.4: Estimativas (erros padrões) dos parâmetros - modelo 3.

Na Tabela 5.4, percebe-se que as estimativas obtidas para os parâmetros associados aos efeitos fixos são exatamente as mesmas obtidas no modelo com apenas o intercepto aleatório. Portanto, as conclusões marginais são as mesmas. O que muda nesse modelo é a inclusão do parâmetro $\sigma_{u 2}^{2}$ na estrutura de covariância.

Nas Figuras 5.20 e 5.21, os gráficos dos resíduos marginais e condicionais padronizados indicam que as suposições de linearidade dos efeitos fixos e de homocedasticidade dos erros 
condicionais são satisfeitas. O gráfico para as estruturas de covariâncias (Figura 5.22) não destaca nenhuma unidade experimental com valor fora da faixa esperada. Os gráficos Q-Q para a distribuição normal (Figuras 5.23 a 5.26) indicam que o modelo está bem ajustado. Apenas o gráfico dos resíduos $t_{i j}^{*}$ apresentou alguns pontos fora da região esperada (Figura 5.25). Na Figura 5.27, novamente os porcos 2 e 5 são considerados discrepantes, mas o gráfico Q-Q da distribuição $\chi_{2}^{2}$ apresenta um resultado melhor do que o obtido no ajuste anterior.

A observação 1 do porco 6 é destacada como influente (Figura 5.28). Sem essa observação, as estimativas são $\hat{\beta}_{1}=196,226$ e $\hat{\beta}_{2}=38,975$, com $\hat{\sigma}^{2}=507,469, \hat{\sigma}_{u 1}^{2}=2716,532$ e $\hat{\sigma}_{u 2}^{2}=81,743$, sem mudança inferencial no ajuste do modelo.

Utilizando os critérios AIC e BIC para comparar os três modelos, aquele que apresenta os melhores resultados é o modelo 1, no qual a estação do ano é uma variável qualitativa (Tabela $5.5)$.

\begin{tabular}{lrr}
\hline & AIC & BIC \\
\hline Modelo 1 & 408,960 & 419,090 \\
Modelo 2 & 415,400 & 422,150 \\
Modelo 3 & 414,260 & 422,700 \\
\hline
\end{tabular}

Tabela 5.5: Comparação entre os três modelos ajustados.
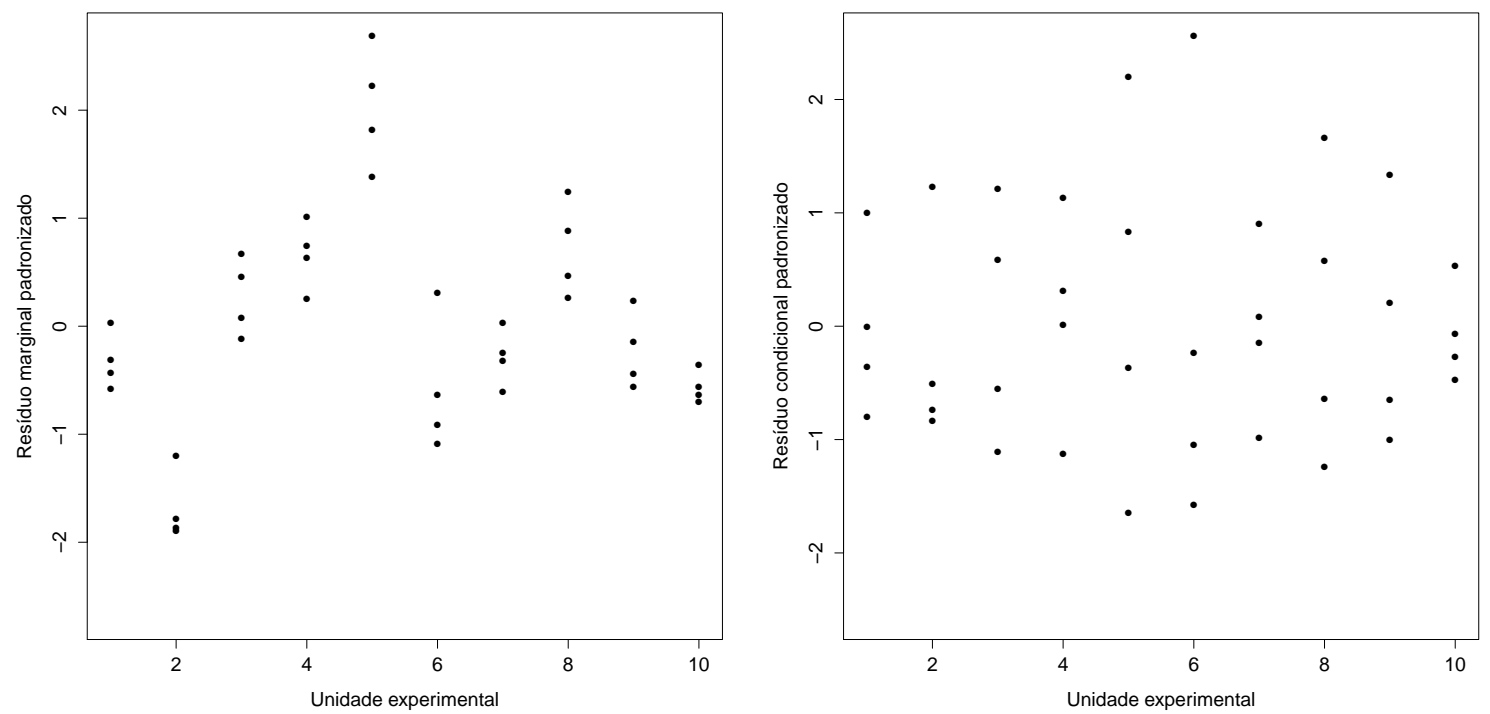

Figura 5.2: Resíduos marginais e condicionais padronizados versus os índices das unidades experimentais - modelo 1 . 

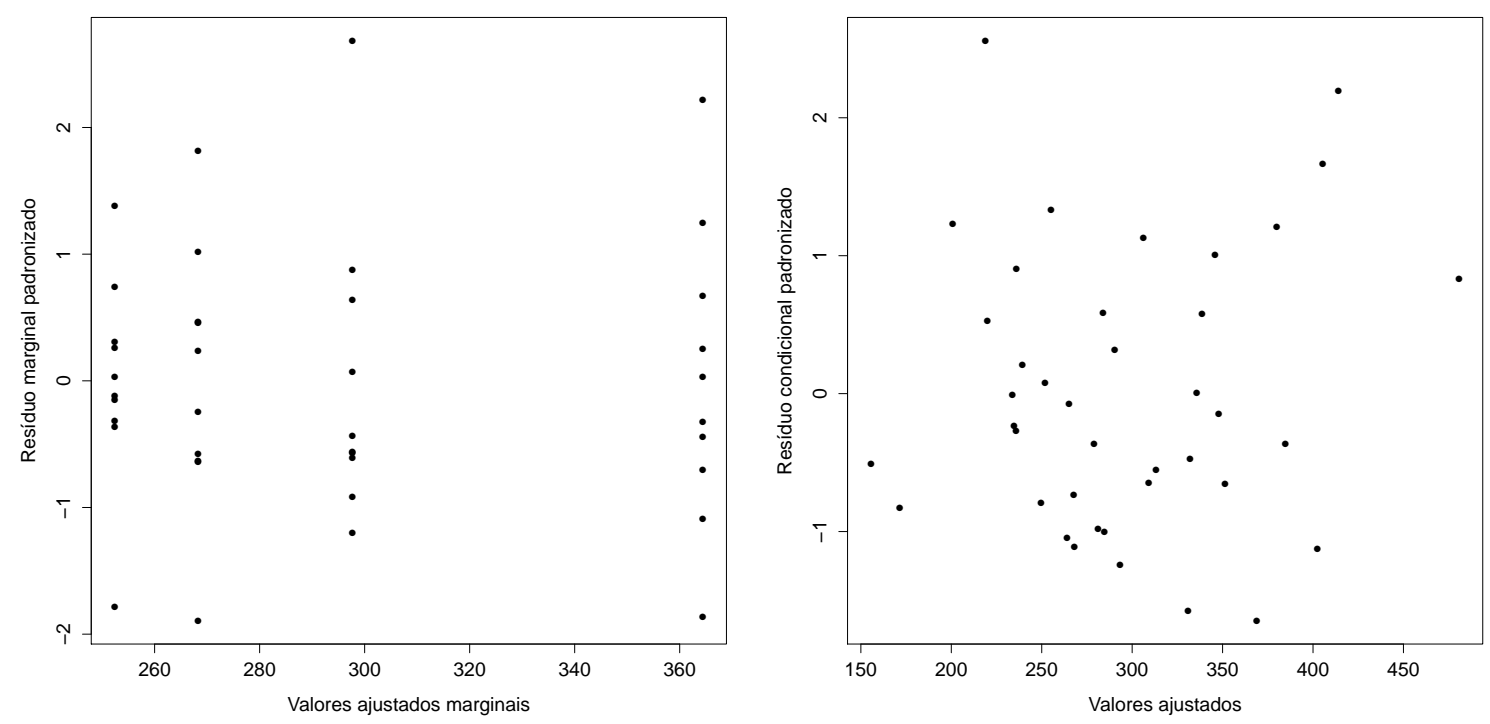

Figura 5.3: Resíduos marginais e condicionais padronizados versus os valores ajustados - modelo 1.

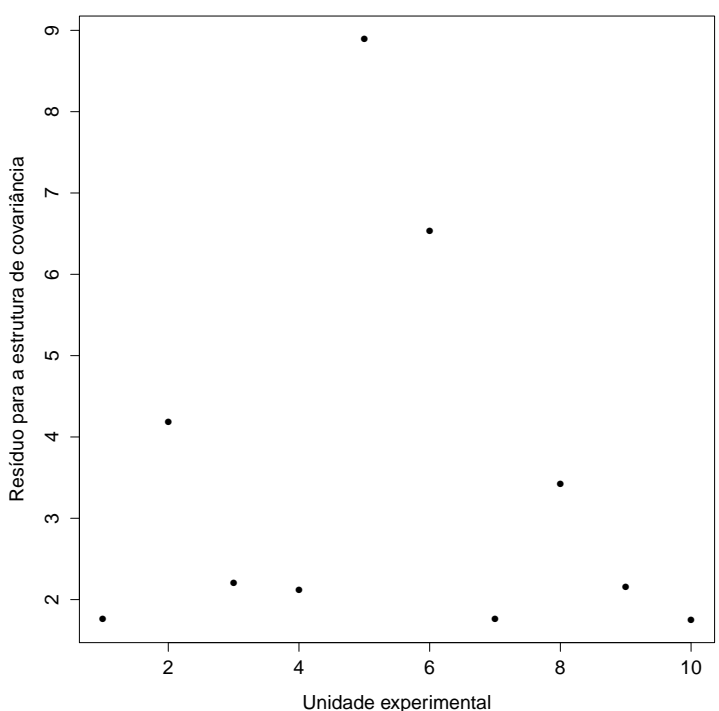

Figura 5.4: Gráfico dos resíduos para a estrutura de covariância versus os índices das unidades experimentais - modelo 1 . 

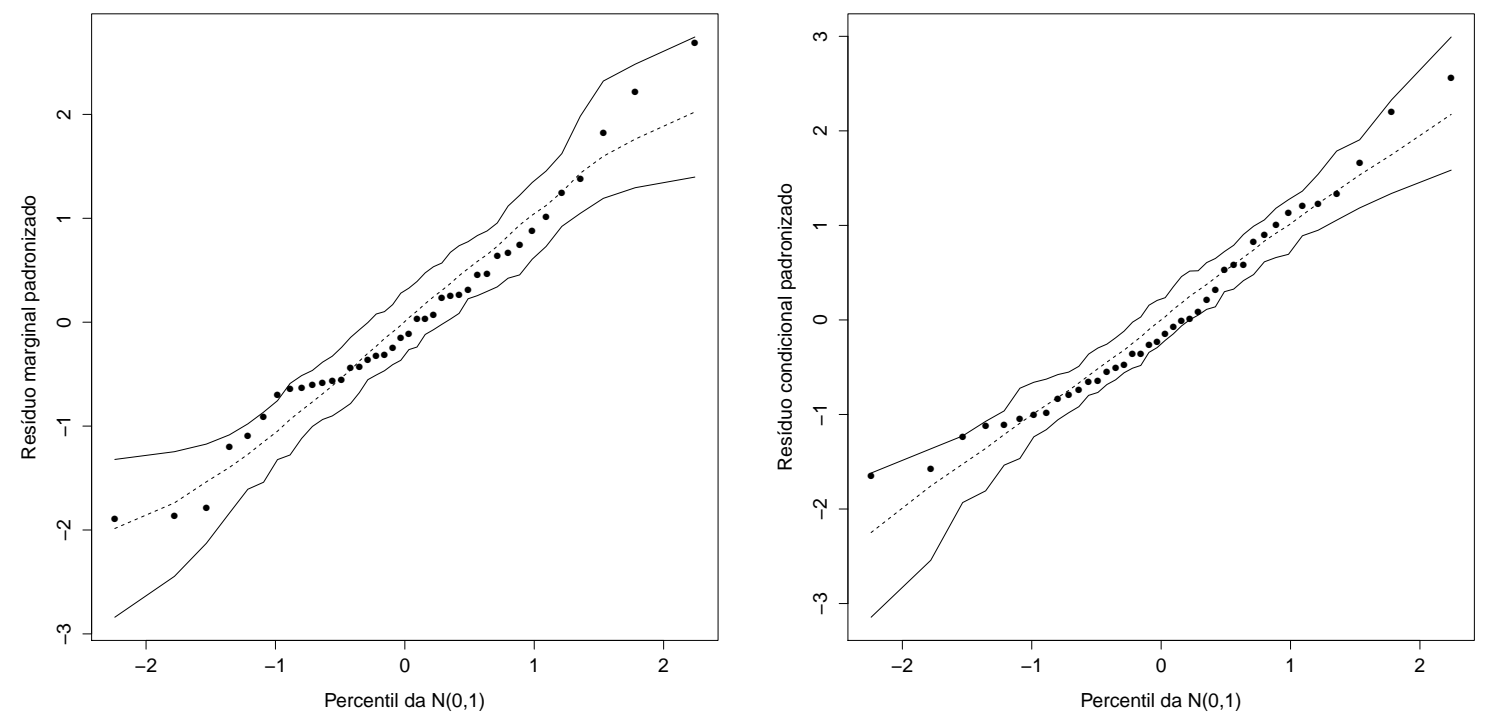

Figura 5.5: Gráficos $Q-Q$ dos resíduos marginais e condicionais padronizados - modelo 1.

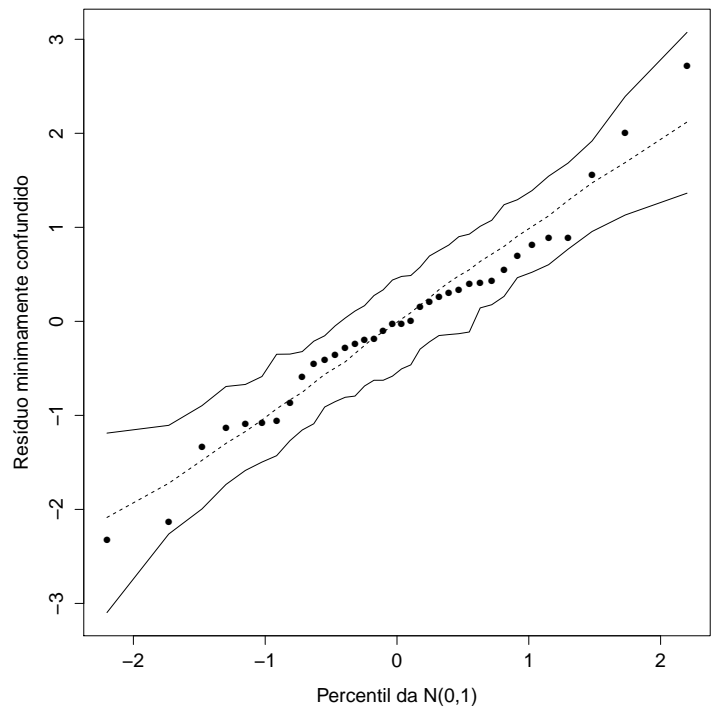

Figura 5.6: Gráfico $Q-Q$ dos resíduos minimamente confundidos - modelo 1. 


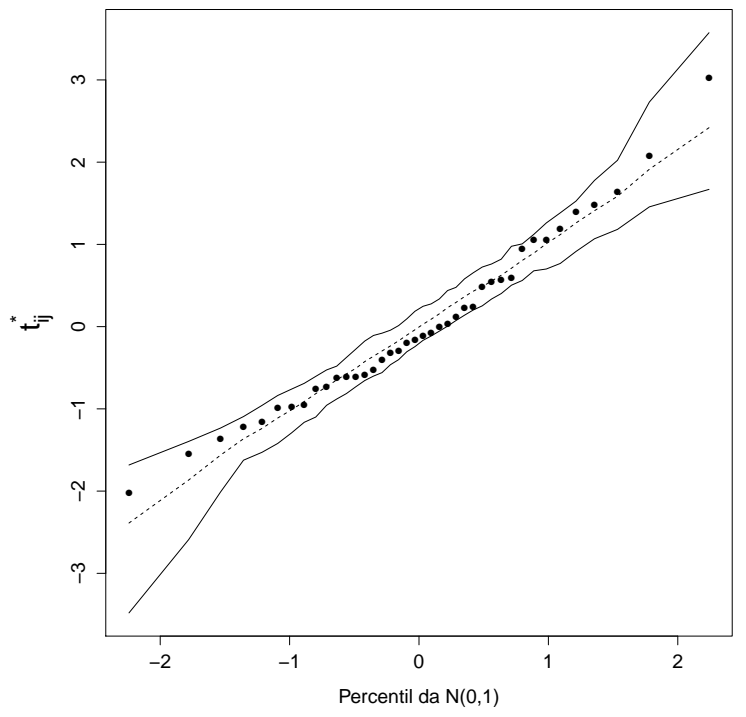

Figura 5.7: Gráfico $Q-Q$ dos resíduos $t_{i j}^{*}$ - modelo 1.

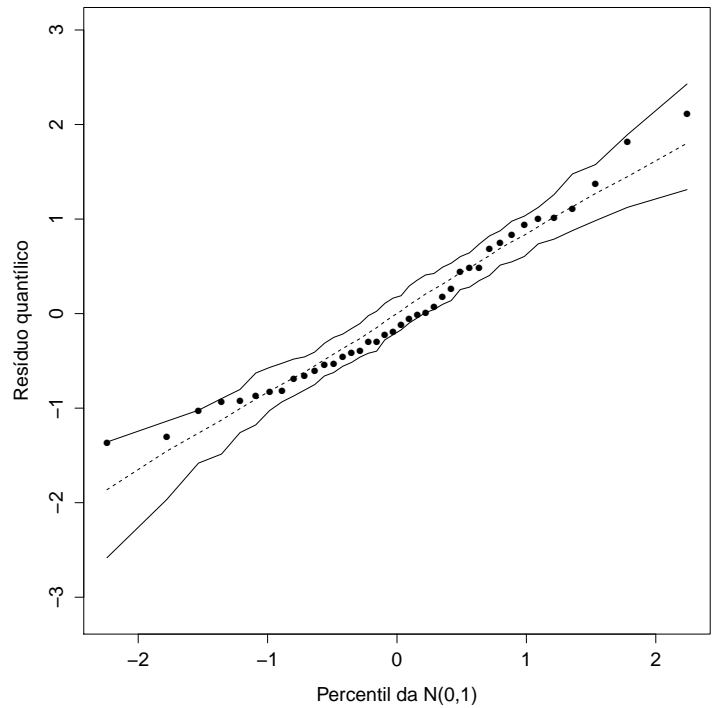

Figura 5.8: Gráfico $Q-Q$ dos resíduos quantílicos - modelo 1. 

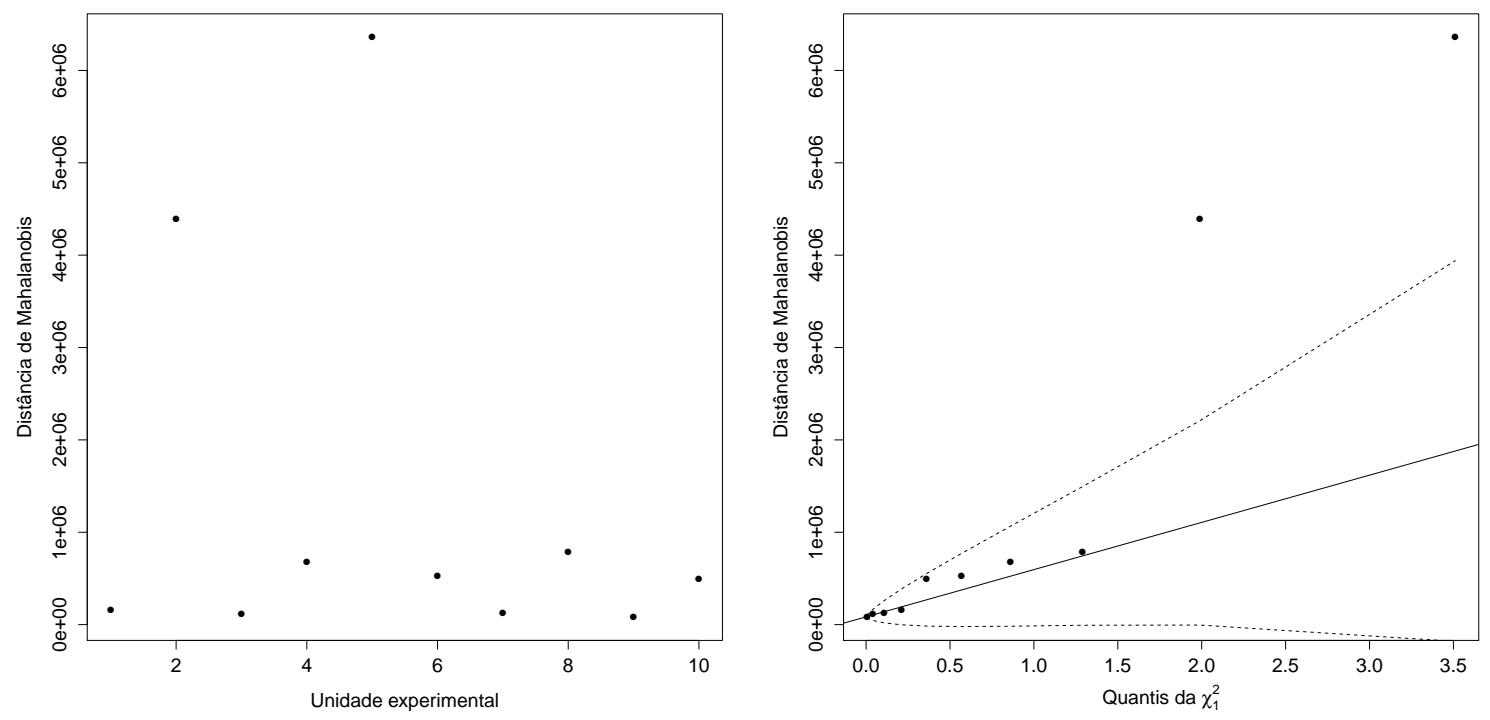

Figura 5.9: Gráficos das distâncias de Mahalanobis - modelo 1.

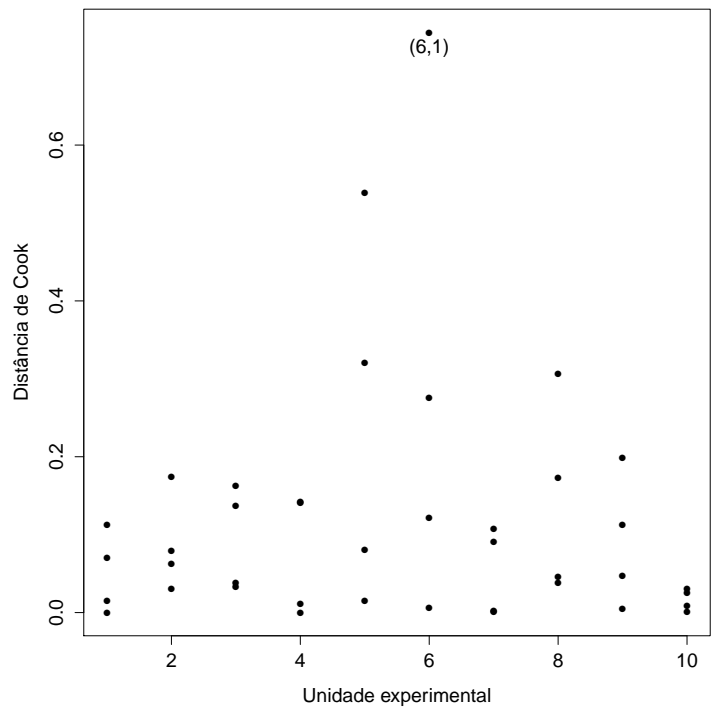

Figura 5.10: Gráfico das distâncias de Cook versus os índices das unidades experimentais - modelo 1. 

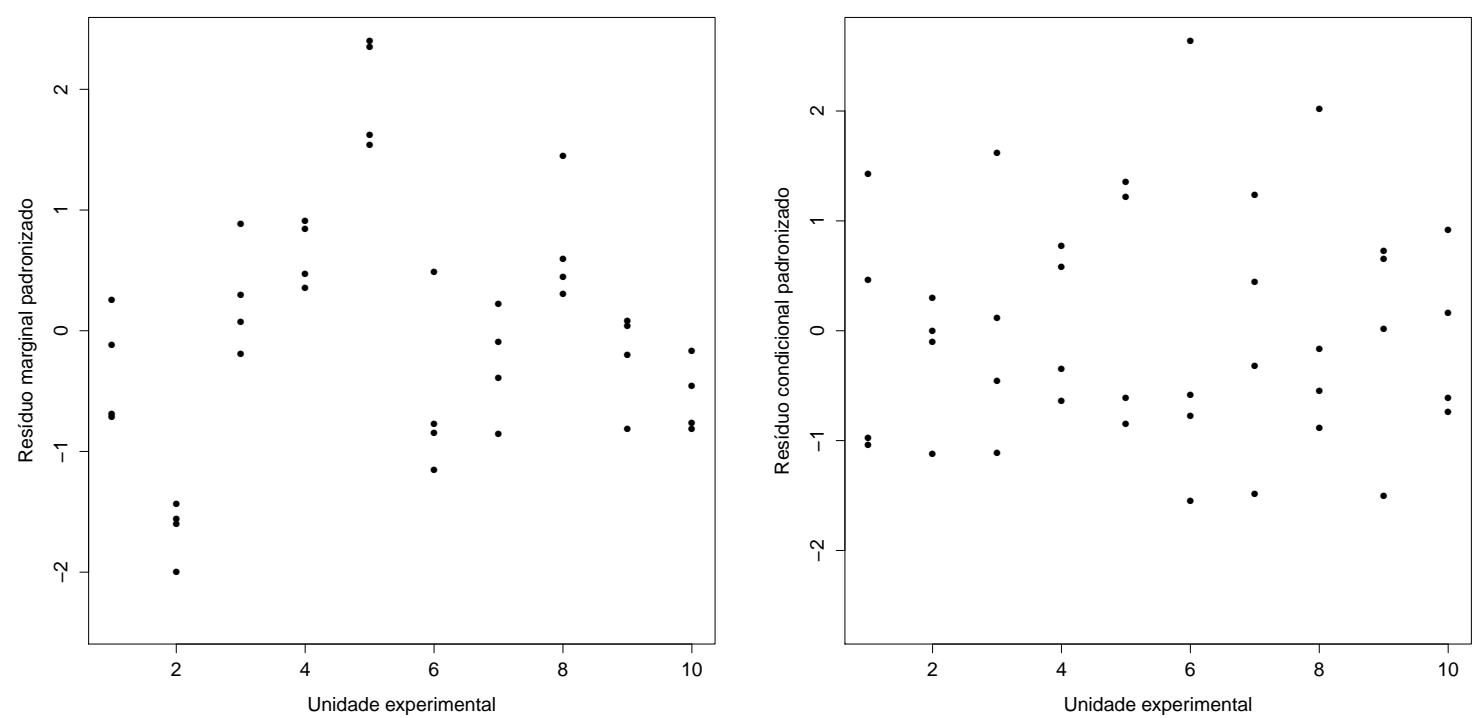

Figura 5.11: Resíduos marginais e condicionais padronizados versus os índices das unidades experimentais - modelo 2 .
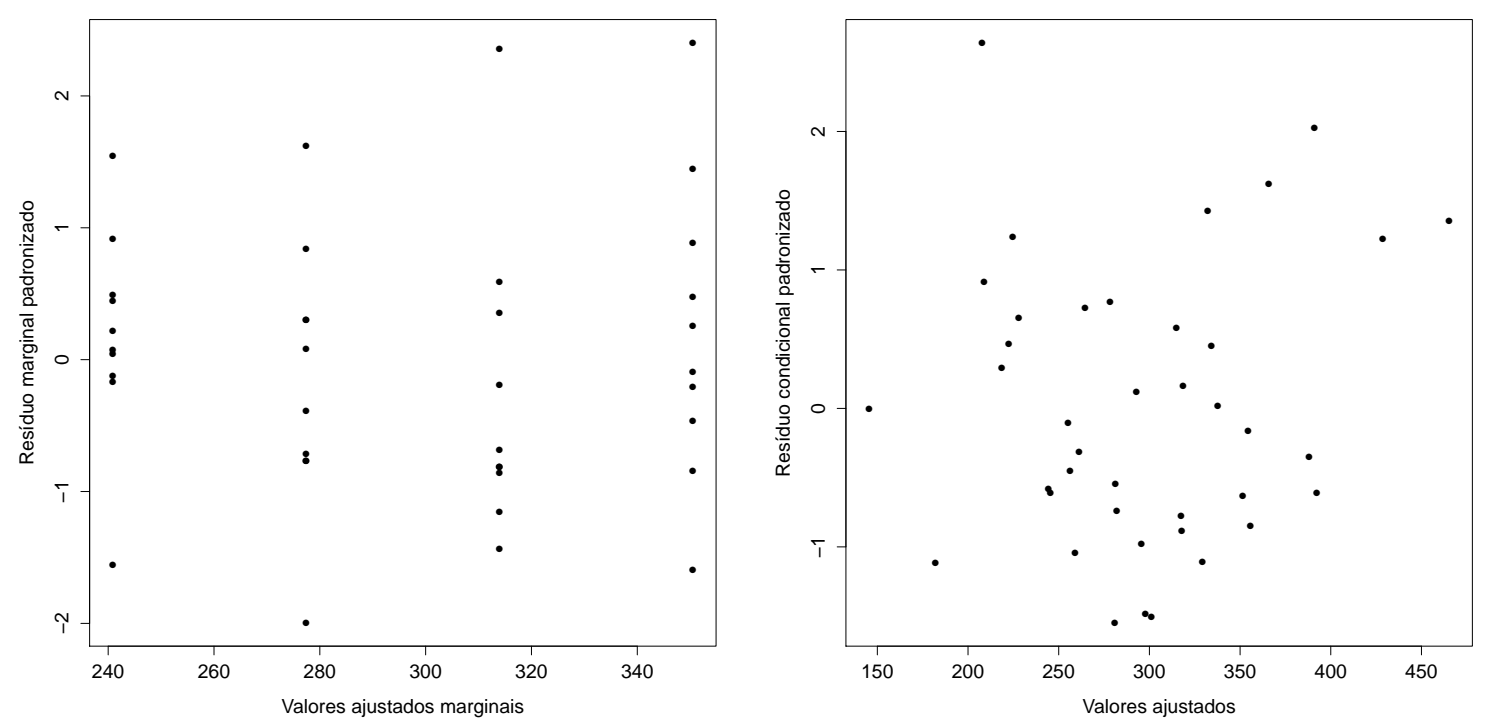

Figura 5.12: Resíduos marginais e condicionais padronizados versus os valores ajustados - modelo 2. 


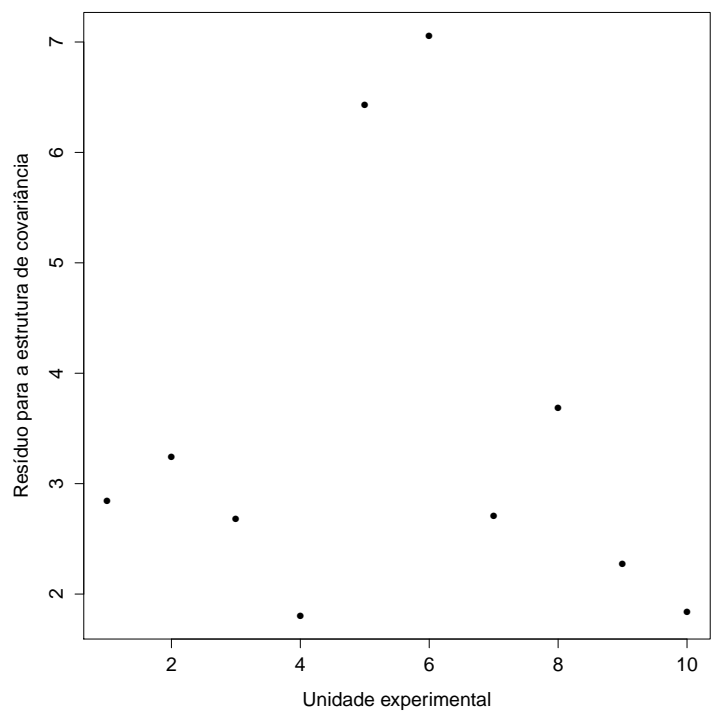

Figura 5.13: Gráfico dos resíduos para a estrutura de covariância versus os índices das unidades experimentais - modelo 2.
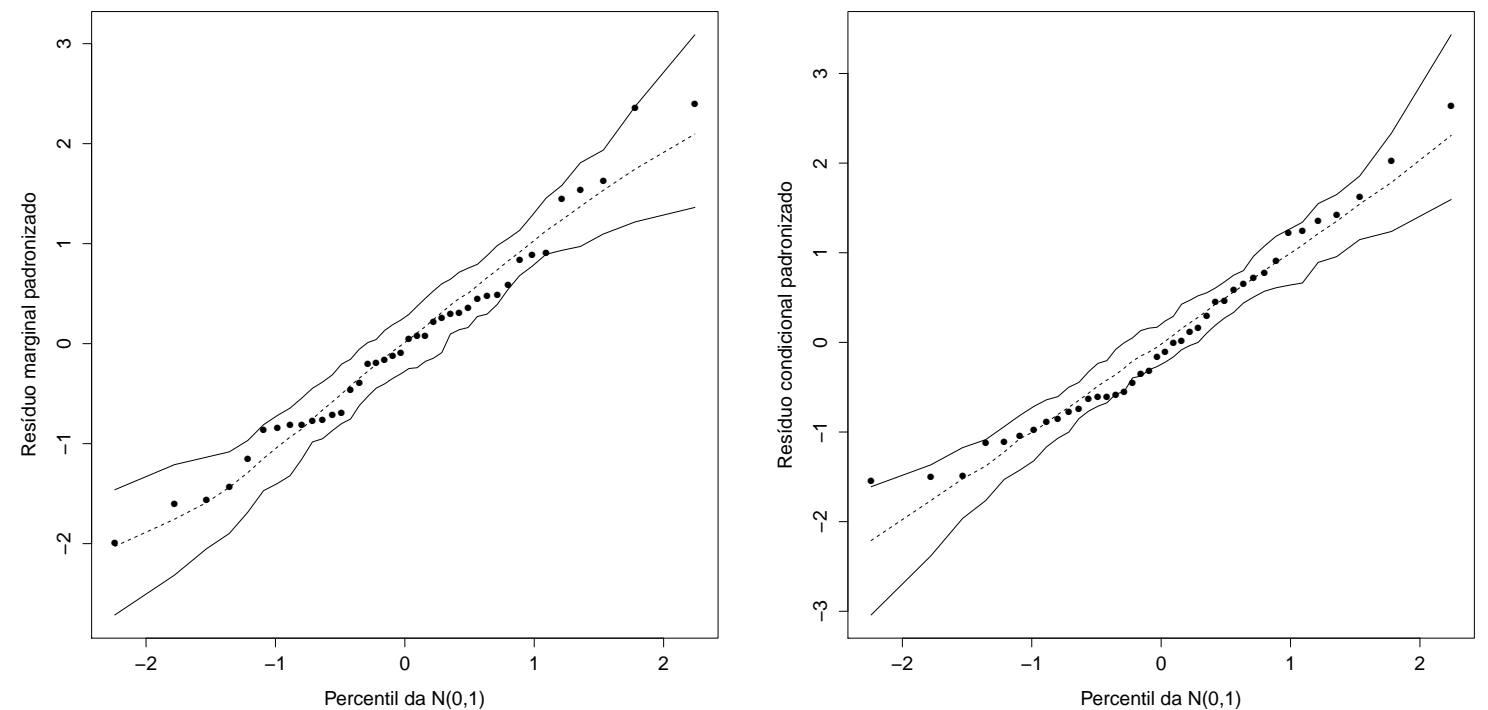

Figura 5.14: Gráficos $Q-Q$ dos resíduos marginais e condicionais padronizados - modelo 2. 


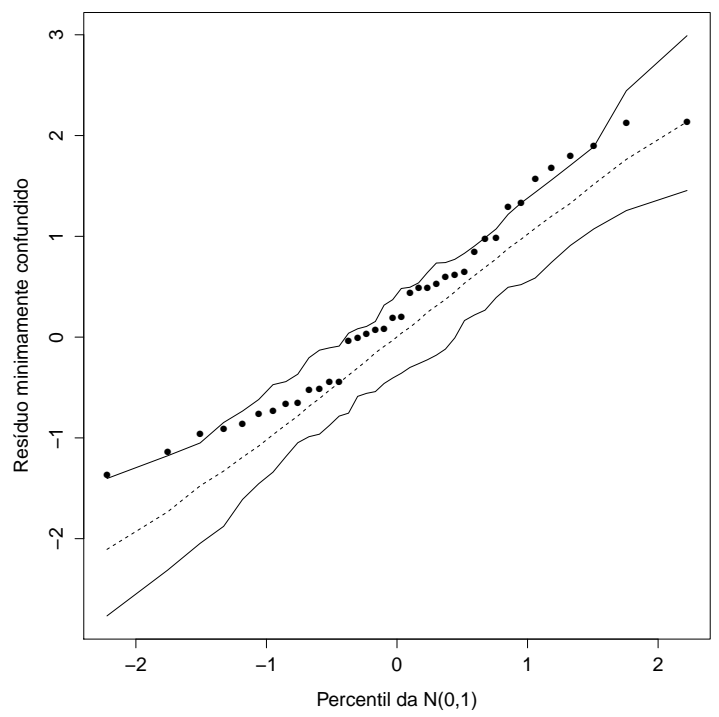

Figura 5.15: Gráfico $Q-Q$ dos resíduos minimamente confundidos - modelo 2.

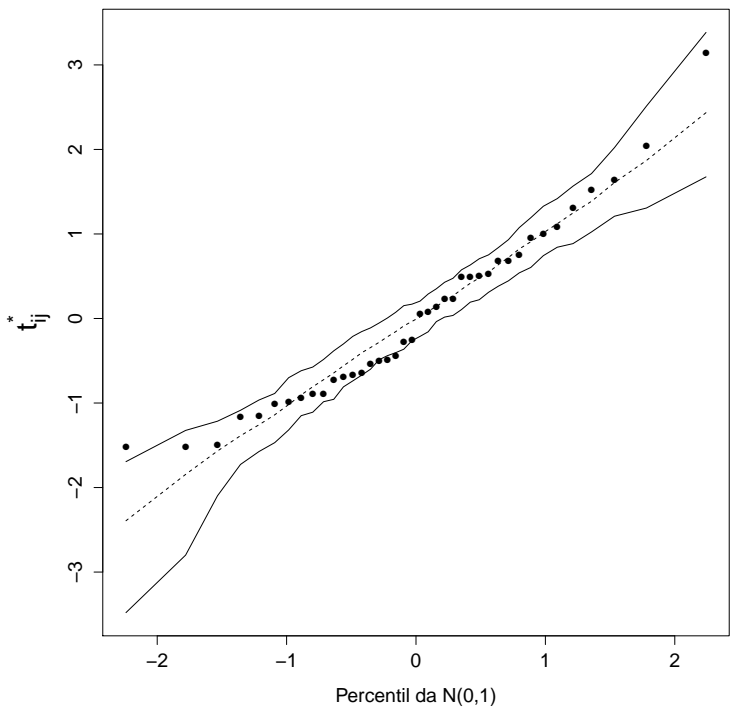

Figura 5.16: Gráfico $Q-Q$ dos resíduos $t_{i j}^{*}$ - modelo 2. 


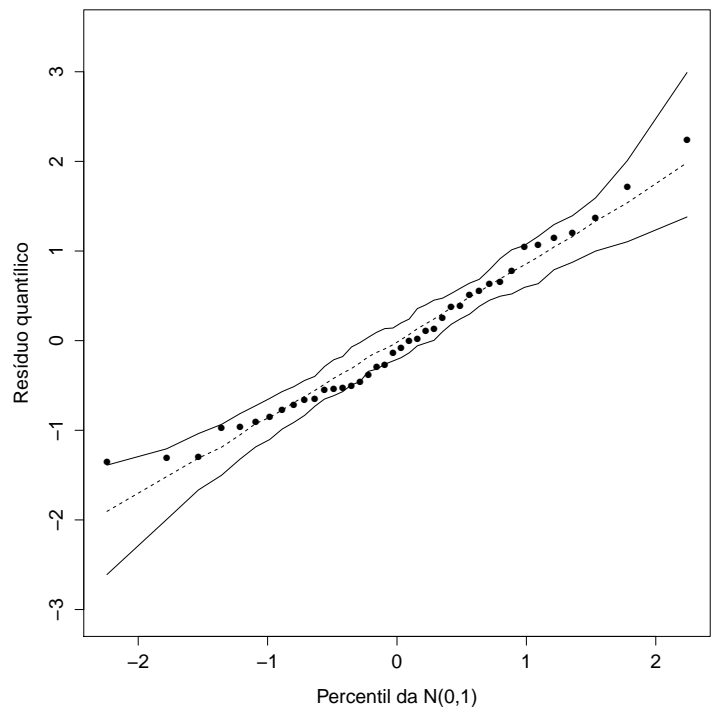

Figura 5.17: Gráfico $Q-Q$ dos resíduos quantílicos - modelo 2.
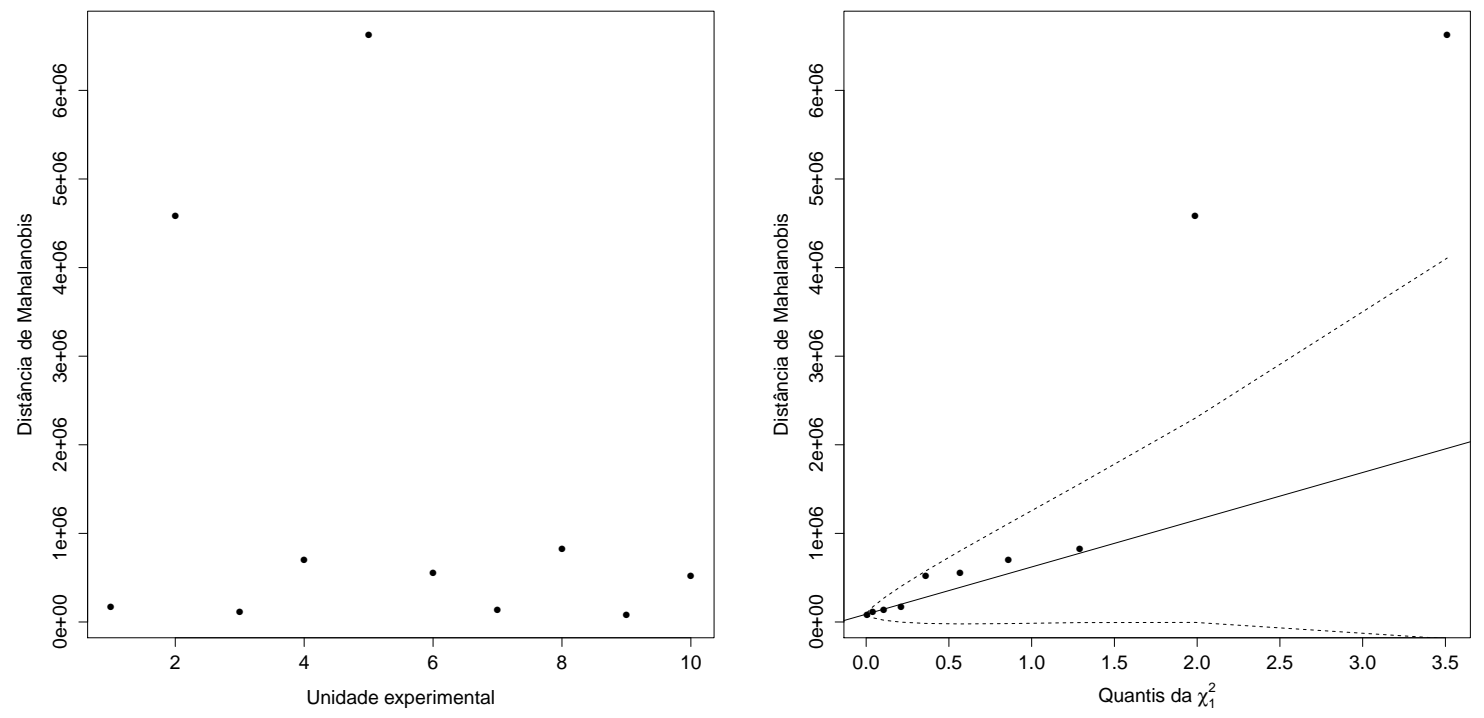

Figura 5.18: Gráficos das distâncias de Mahalanobis - modelo 2. 


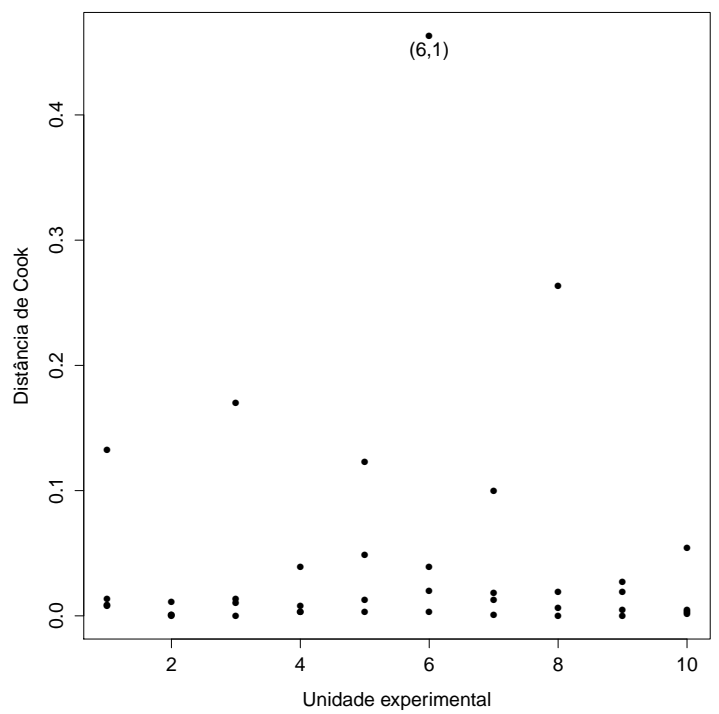

Figura 5.19: Gráfico das distâncias de Cook versus os indices das unidades experimentais - modelo 2.
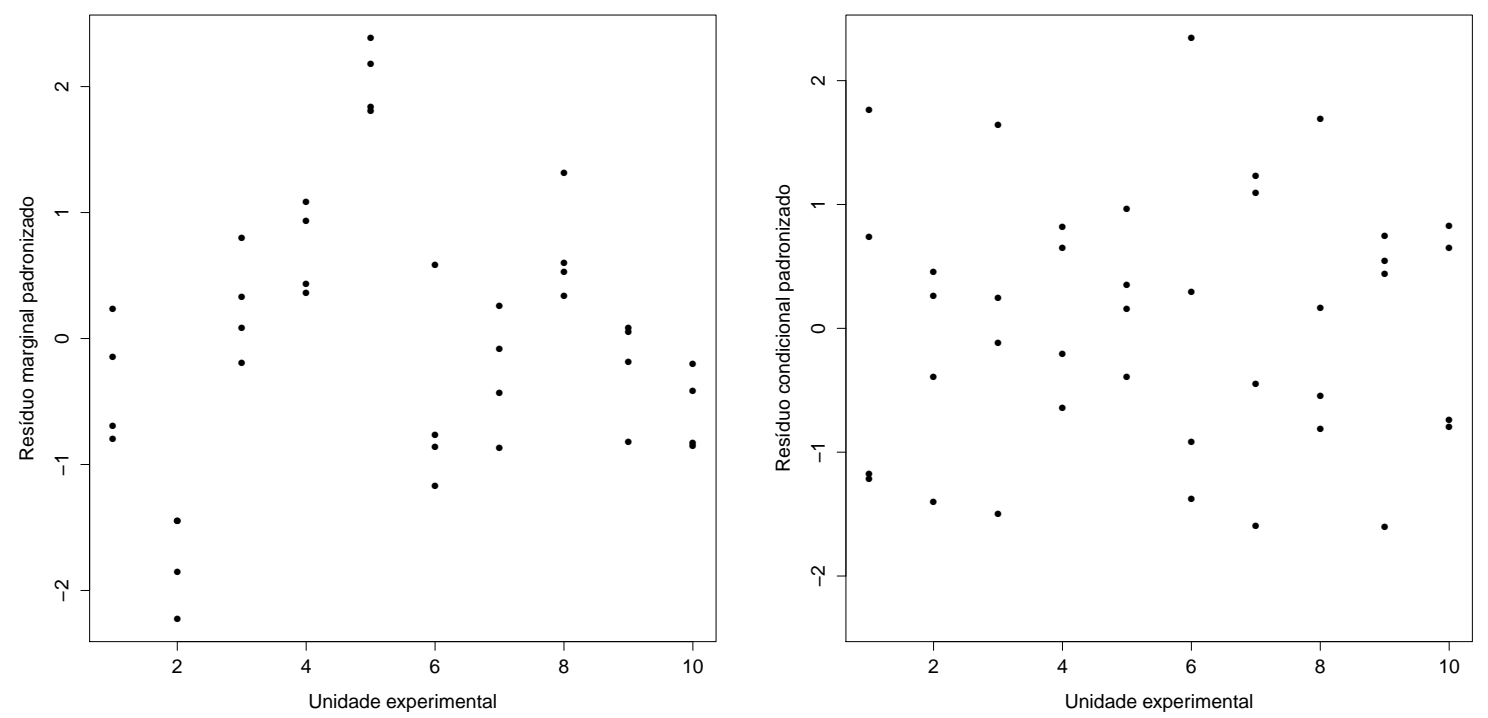

Figura 5.20: Resíduos marginais e condicionais padronizados versus os índices das unidades experimentais - modelo 3 . 

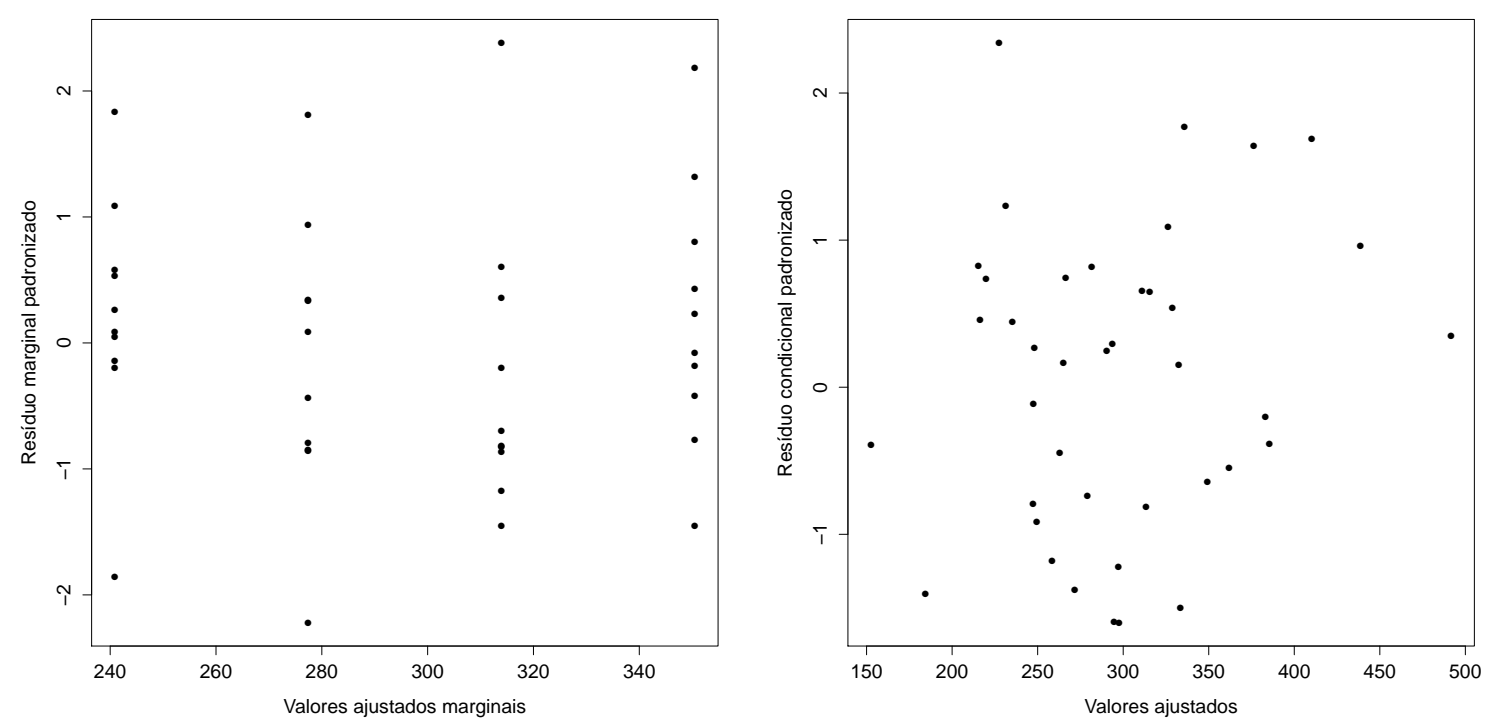

Figura 5.21: Resíduos marginais e condicionais padronizados versus os valores ajustados - modelo 3.

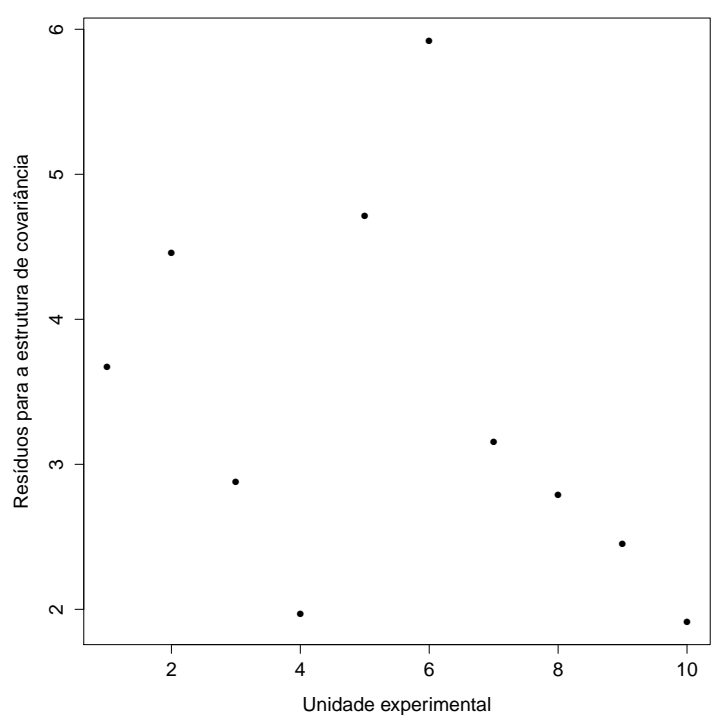

Figura 5.22: Gráfico dos resíduos para a estrutura de covariância versus os índices das unidades experimentais - modelo 3. 

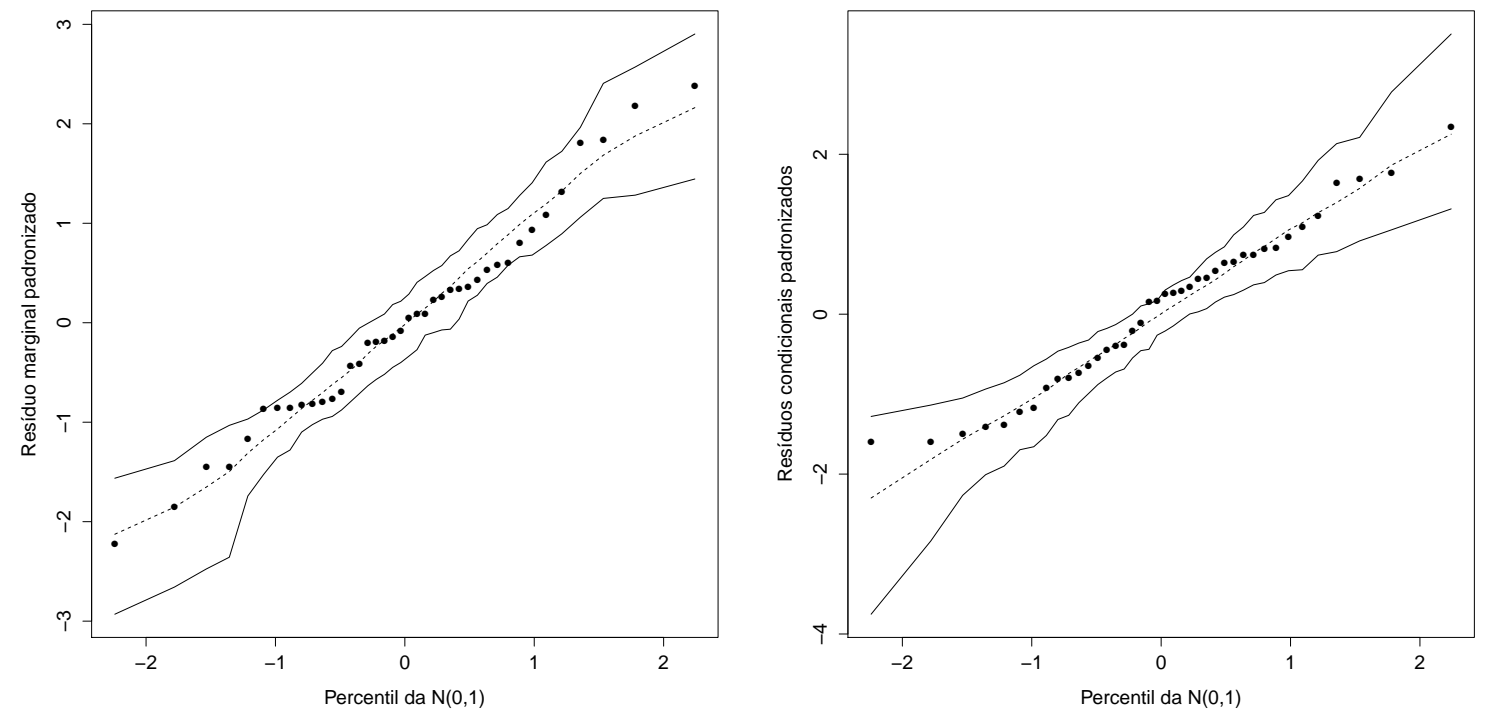

Figura 5.23: Gráficos $Q-Q$ dos resíduos marginais e condicionais padronizados - modelo 3.

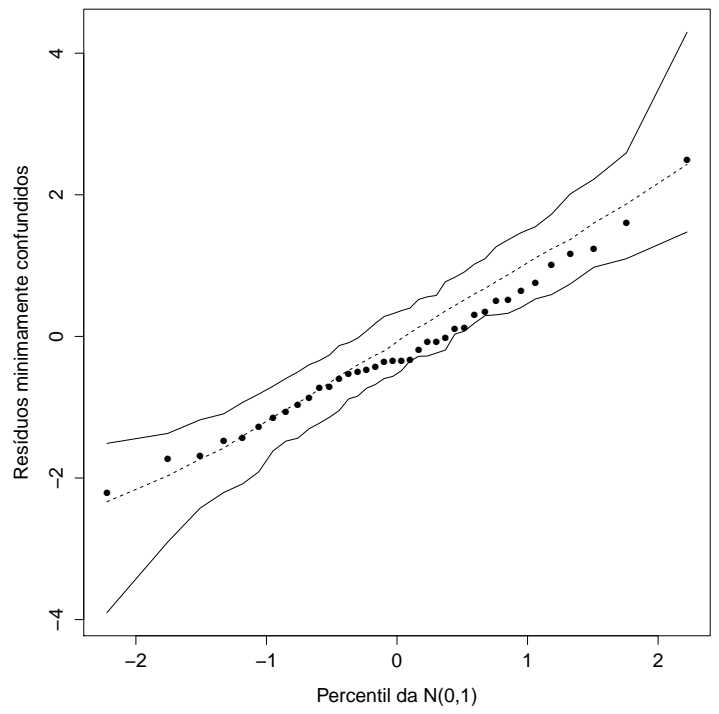

Figura 5.24: Gráfico $Q-Q$ dos resíduos minimamente confundidos - modelo 3. 


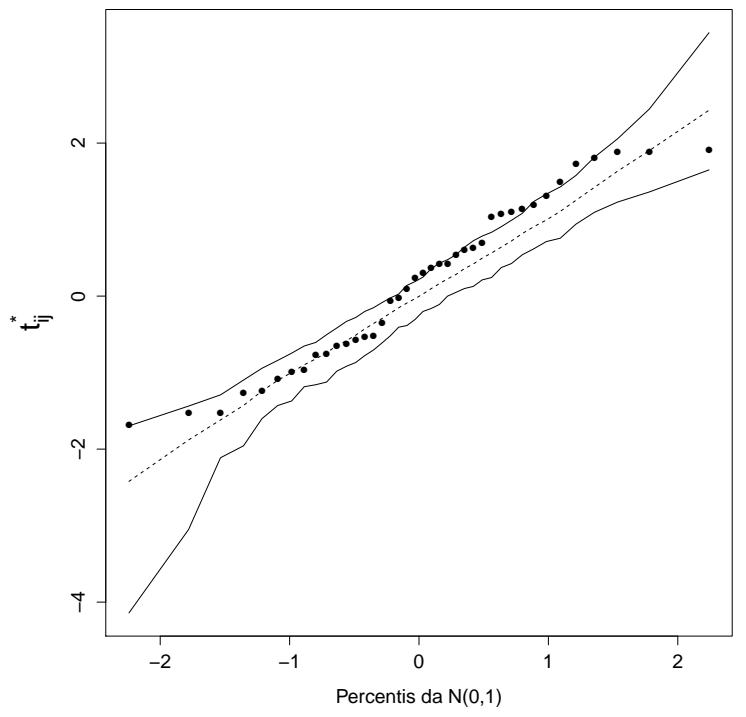

Figura 5.25: Gráfico $Q-Q$ dos resíduos $t_{i j}^{*}$ - modelo 3.

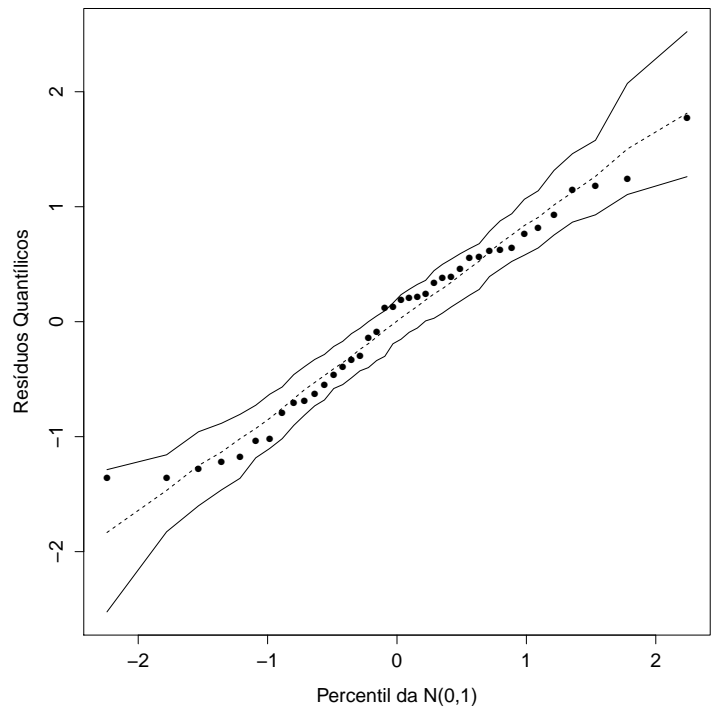

Figura 5.26: Gráfico $Q-Q$ dos resíduos quantílicos - modelo 3. 

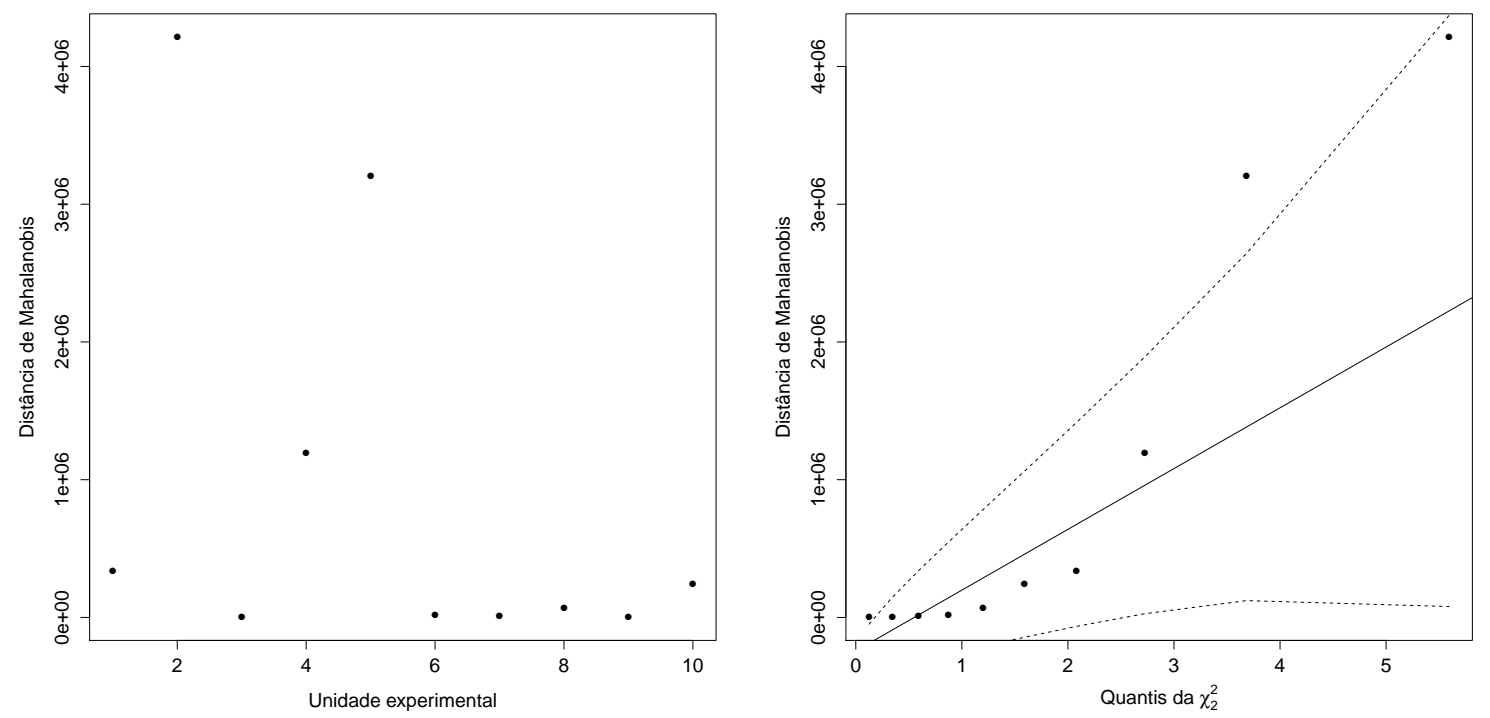

Figura 5.27: Gráficos das distâncias de Mahalanobis - modelo 3.

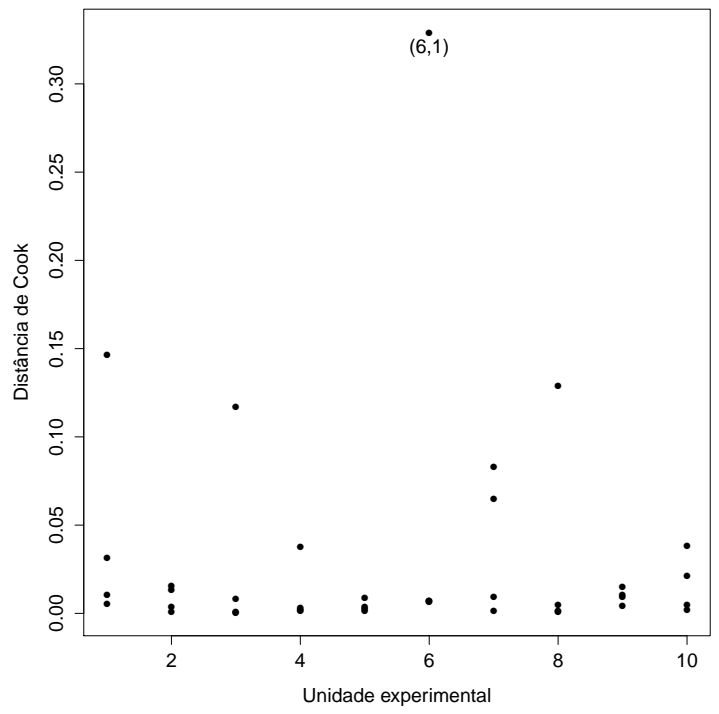

Figura 5.28: Gráfico das distâncias de Cook versus os indices das unidades experimentais - modelo 3. 


\section{Capítulo 6}

\section{Conclusões}

Este trabalho apresentou e avaliou as técnicas de diagnóstico disponíveis na análise de dados com medidas repetidas, de acordo com as metodologias de modelos condicionais e de modelos marginais.

Alguns pontos importantes devem ser considerados a partir dos resultados obtidos nos estudos de simulação. São eles:

- Quando a distribuição condicional da variável resposta é normal, a distribuição dos efeitos aleatórios não é normal e é ajustado um modelo linear misto, o gráfico Q-Q das distâncias de Mahalanobis detecta a inadequação da distribuição dos efeitos aleatórios. No entanto, as estimativas obtidas para os efeitos fixos não se alteram. Isso reforça o que foi apresentado em trabalhos como Butler e Louis [1992], Verbeke e Lesaffre [1996, 1997] e McCulloch e Neuhaus [2011], que afirmam que as estimativas desses parâmetros em modelos com intercepto aleatório são consistentes mesmo quando a distribuição dos efeitos aleatórios é especificada de maneira errada.

- Quando a distribuição condicional da variável resposta é normal mas é ajustado um modelo linear misto ou um modelo marginal normal, todos os gráficos de diagnóstico indicam, em geral, que o modelo normal não é adequado. Deve ser ressaltado que o gráfico dos resíduos minimamente confundidos pode indicar indevidamente um bom ajuste.

- O diagnóstico em modelos ajustados com intercepto aleatório e em modelos marginais indica um mau ajuste quando é gerado um conjunto de dados a partir de um modelo com intercepto e inclinação aleatórios.

- Quando é violada a suposição de independência condicional no modelo misto, não há grande impacto nas estimativas dos efeitos fixos e nem na qualidade geral do modelo ajustado. Além disso, a violação de suposição não é facilmente detectada pelas técnicas de diagnóstico.

Estudos futuros podem ser feitos para a definição e implementação de mais técnicas de diagnóstico para os modelos lineares generalizados mistos e hierárquicos, já que não há muitas disponíveis nesses casos. Para esses modelos, medidas de influência também podem ser exploradas. 
CONCLUSÕES 


\section{Apêndice A}

\section{Rotinas computacionais}

Para o ajuste dos modelos, os dados da Tabela 5.1 foram dispostos na forma:

\begin{tabular}{|c|c|c|c|}
\hline animal & estnum & vol & est \\
\hline 1 & 1 & 233,571 & Inverno \\
\hline 1 & 2 & 233,462 & Primavera \\
\hline 1 & 3 & 271,667 & Verao \\
\hline 1 & 4 & 366,154 & Outono \\
\hline 2 & 1 & 145,357 & Inverno \\
\hline 2 & 2 & 154,615 & Primavera \\
\hline 2 & 3 & 225,833 & Verao \\
\hline 2 & 4 & 252,692 & Outono \\
\hline 3 & 1 & 245,417 & Inverno \\
\hline 3 & 2 & 295,769 & Primavera \\
\hline 3 & 3 & 302,083 & Verao \\
\hline 3 & 4 & 404,615 & Outono \\
\hline 4 & 1 & 296,786 & Inverno \\
\hline 4 & 2 & 329,231 & Primavera \\
\hline 4 & 3 & 335,833 & Verao \\
\hline 4 & 4 & 379,615 & Outono \\
\hline 5 & 1 & 335,357 & Inverno \\
\hline 5 & 2 & 377,308 & Primavera \\
\hline 5 & 3 & 458,750 & Verao \\
\hline 5 & 4 & 497,692 & Outono \\
\hline 6 & 1 & 270,846 & Inverno \\
\hline 6 & 2 & 230,000 & Primavera \\
\hline 6 & 3 & 242,857 & Verao \\
\hline 6 & 4 & 298,846 & Outono \\
\hline 7 & 1 & 254,286 & Inverno \\
\hline 7 & 2 & 253,462 & Primavera \\
\hline 7 & 3 & 261,250 & Verao \\
\hline 7 & 4 & 345,000 & Outono \\
\hline 8 & 1 & 268,214 & Inverno \\
\hline
\end{tabular}




$\begin{array}{llll}8 & 2 & 296,154 & \text { Primavera } \\ 8 & 3 & 350,417 & \text { Verao } \\ 8 & 4 & 439,231 & \text { Outono } \\ 9 & 1 & 243,571 & \text { Inverno } \\ 9 & 2 & 282,308 & \text { Primavera } \\ 9 & 3 & 264,167 & \text { Verao } \\ 9 & 4 & 338,077 & \text { Outono } \\ 10 & 1 & 230,714 & \text { Inverno } \\ 10 & 2 & 230,385 & \text { Primavera } \\ 10 & 3 & 263,750 & \text { Verao } \\ 10 & 4 & 322,308 & \text { Outono }\end{array}$

\section{Rotinas desenvolvidas no $\mathbf{R}$}

\section{Modelo normal com intercepto aleatório:}

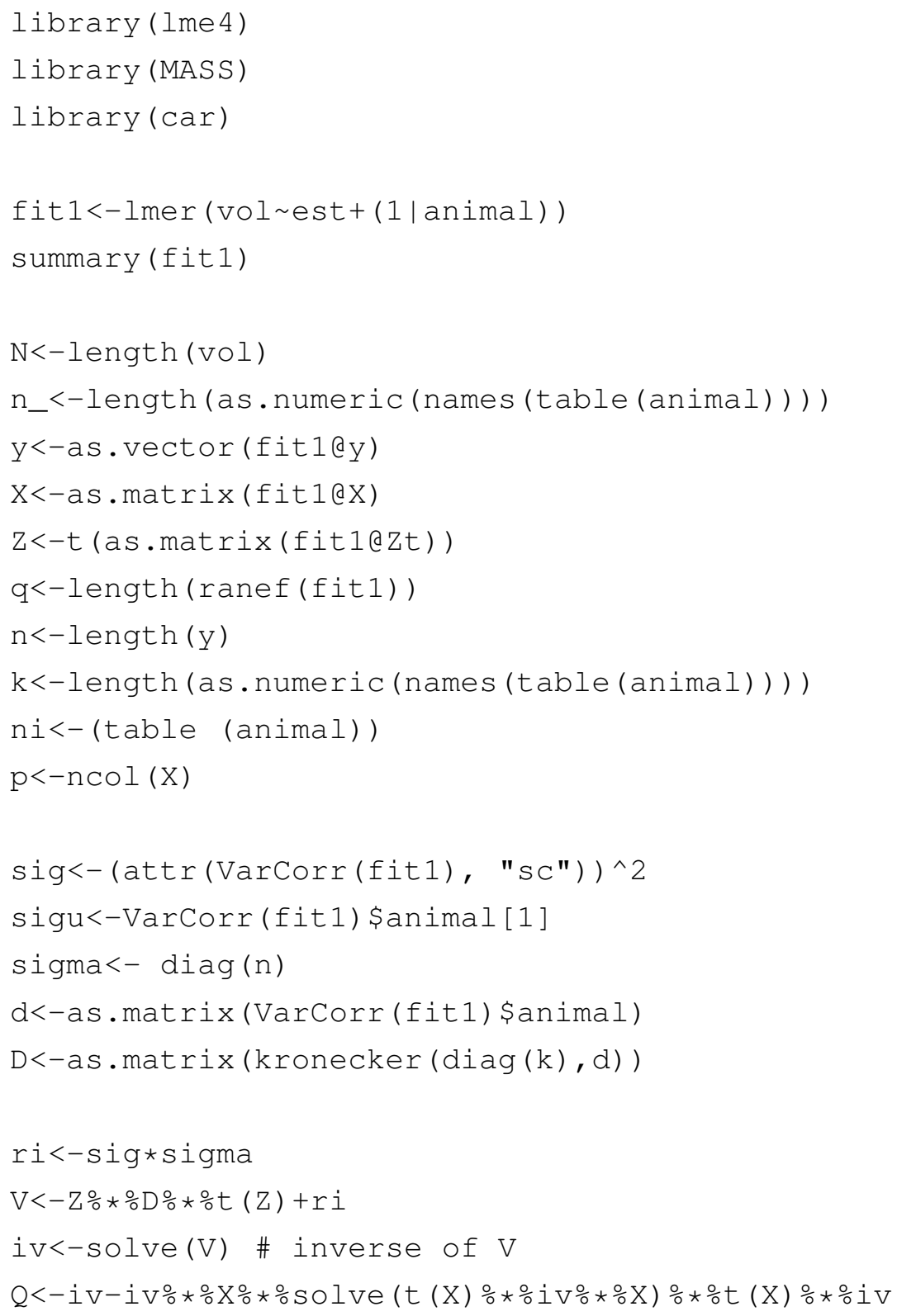




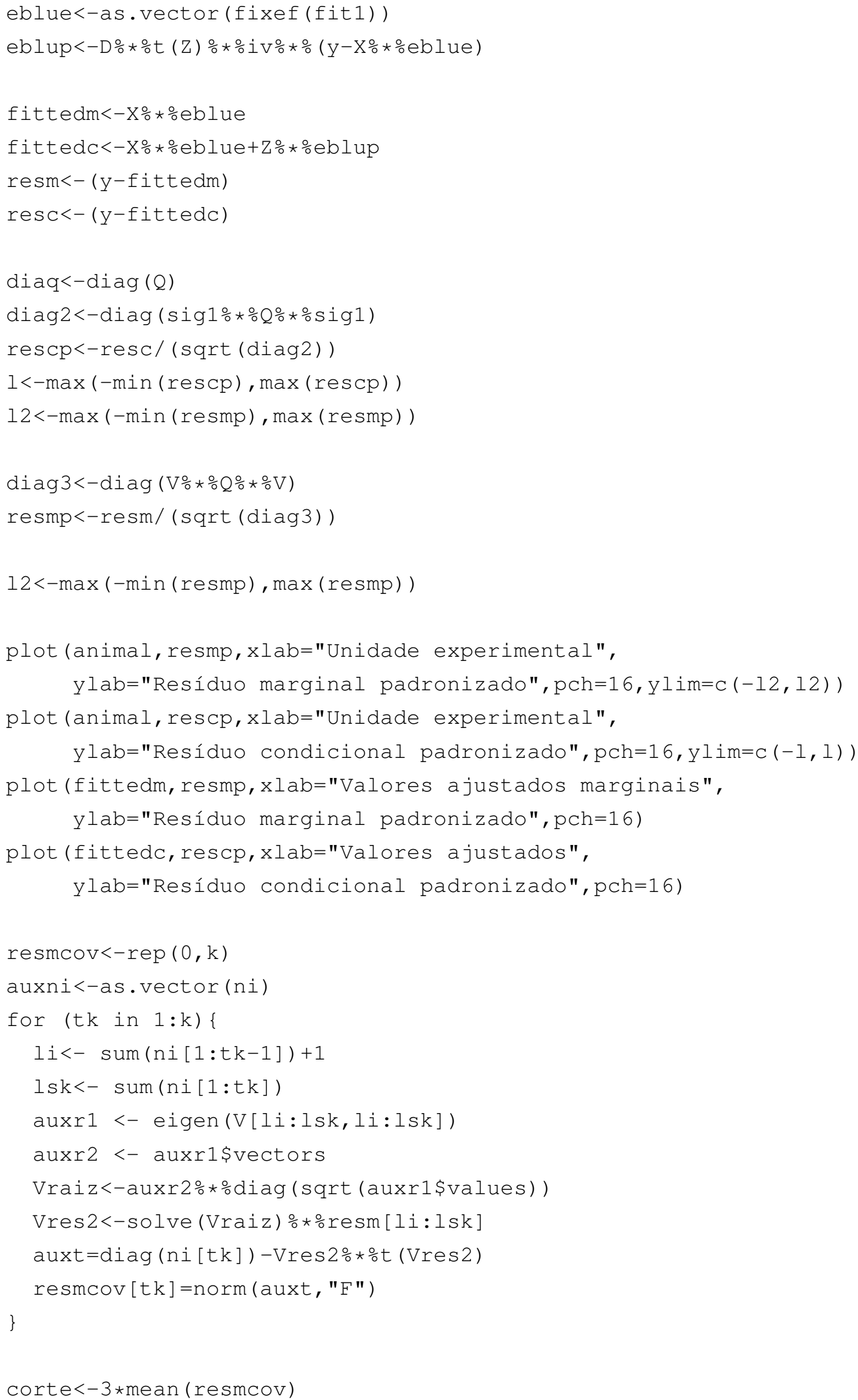




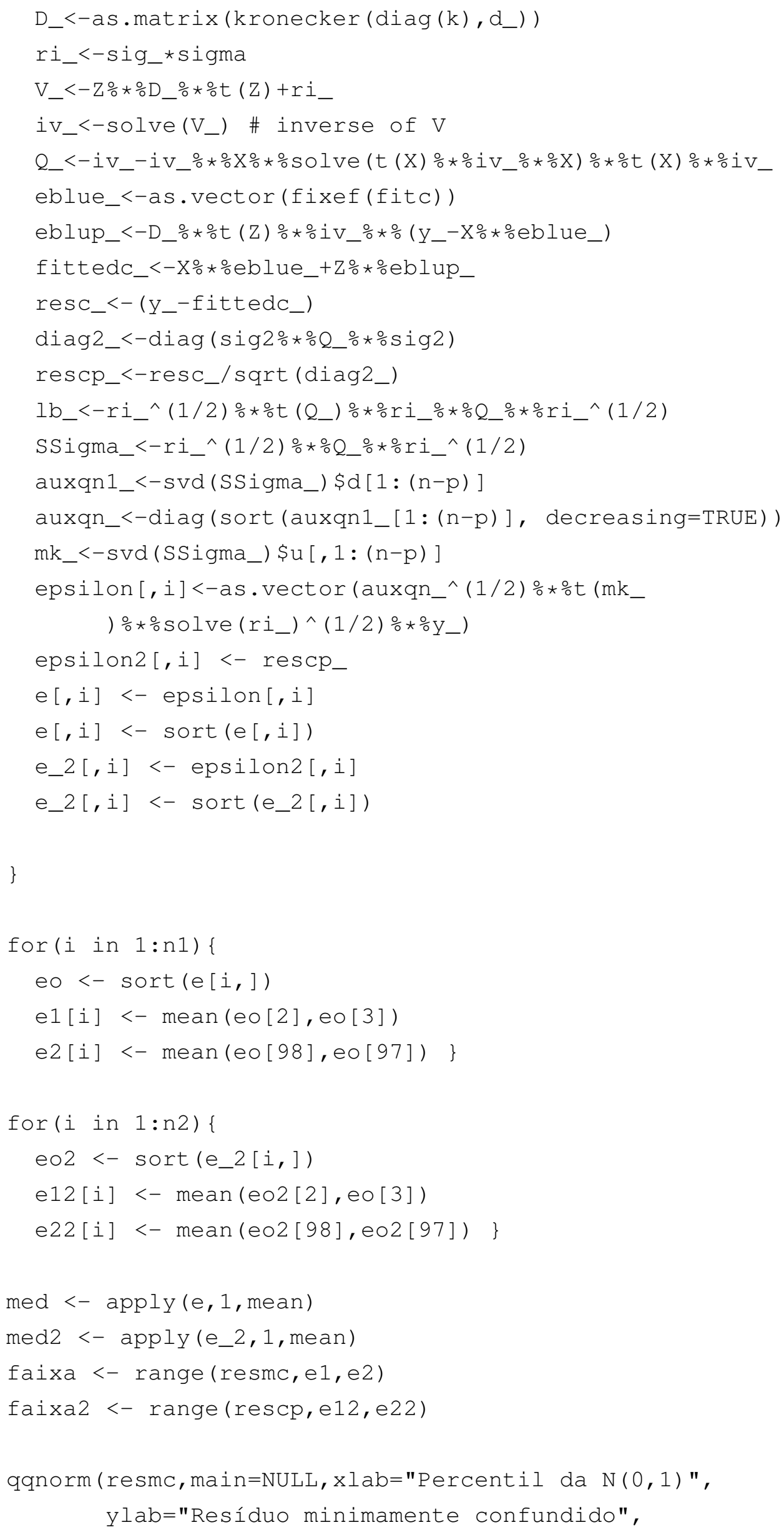




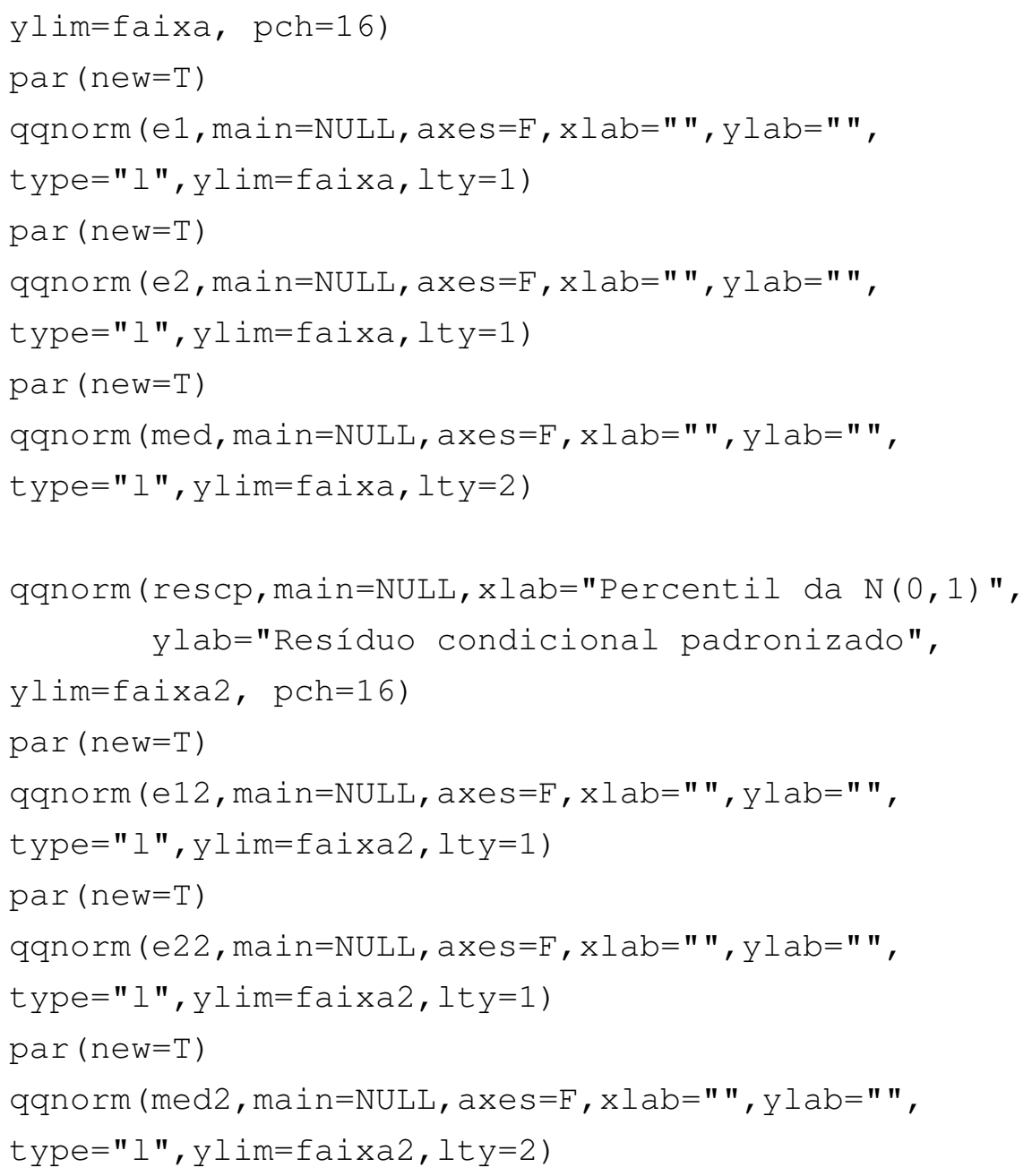




\section{GEE normal:}

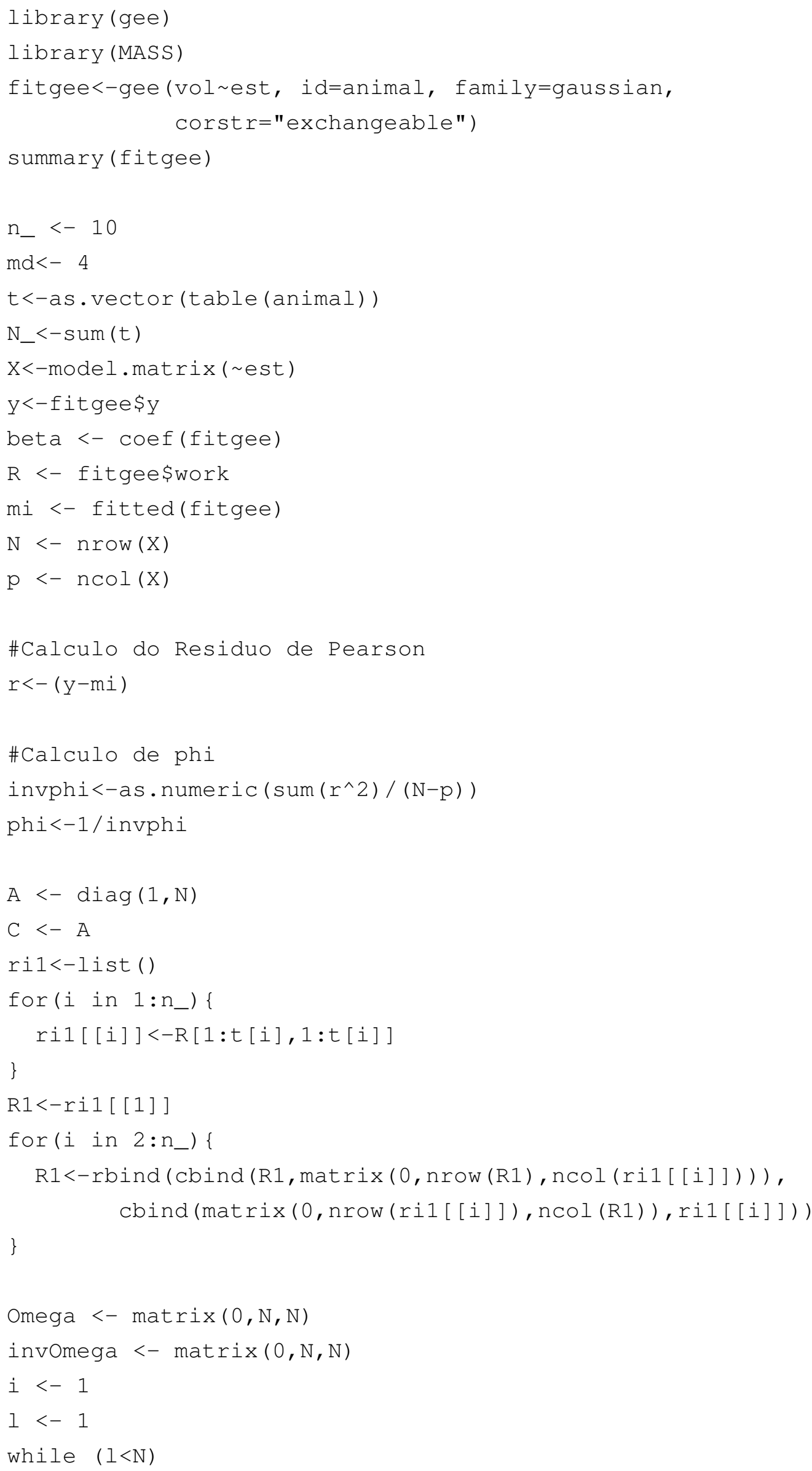




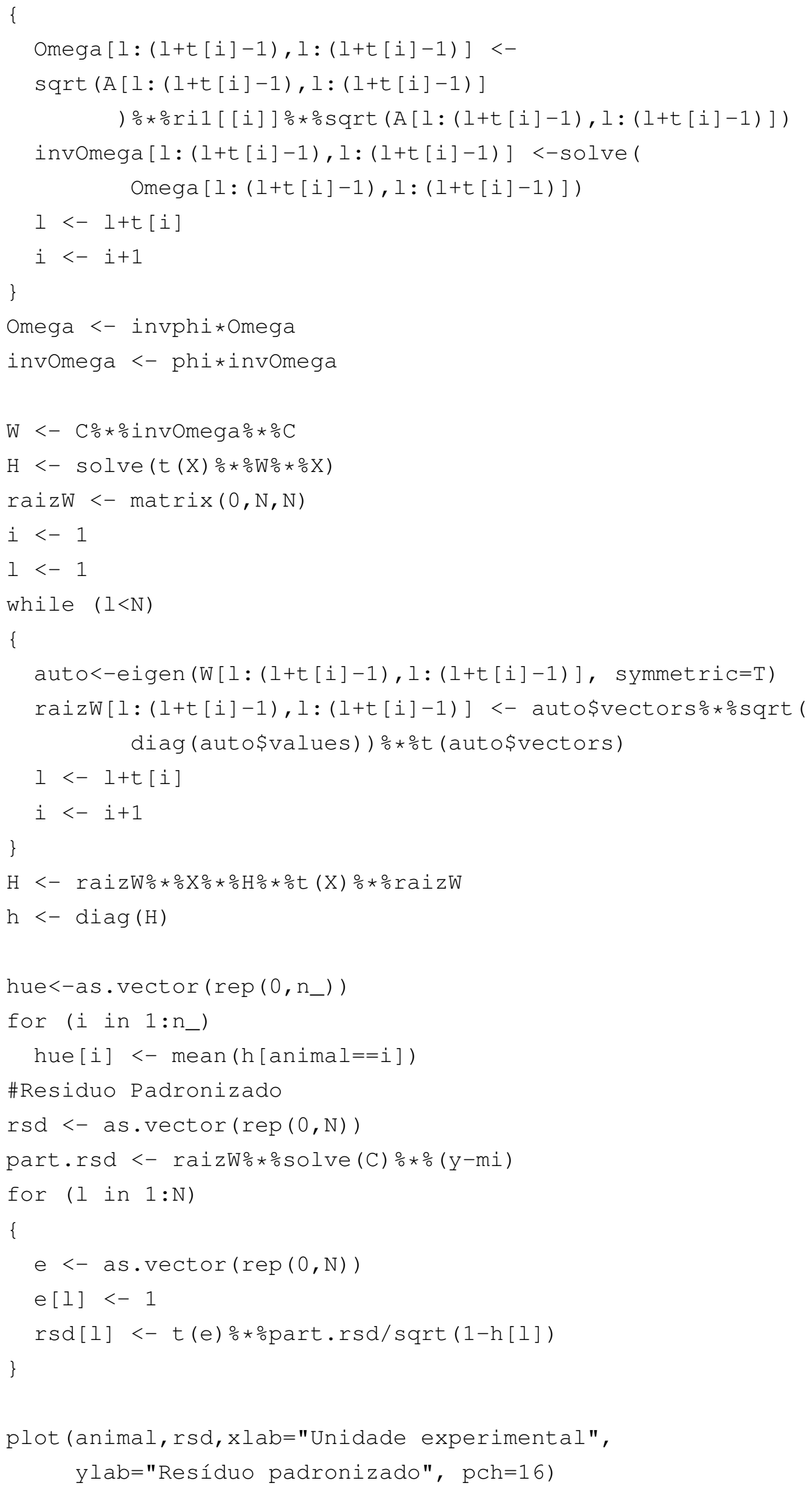




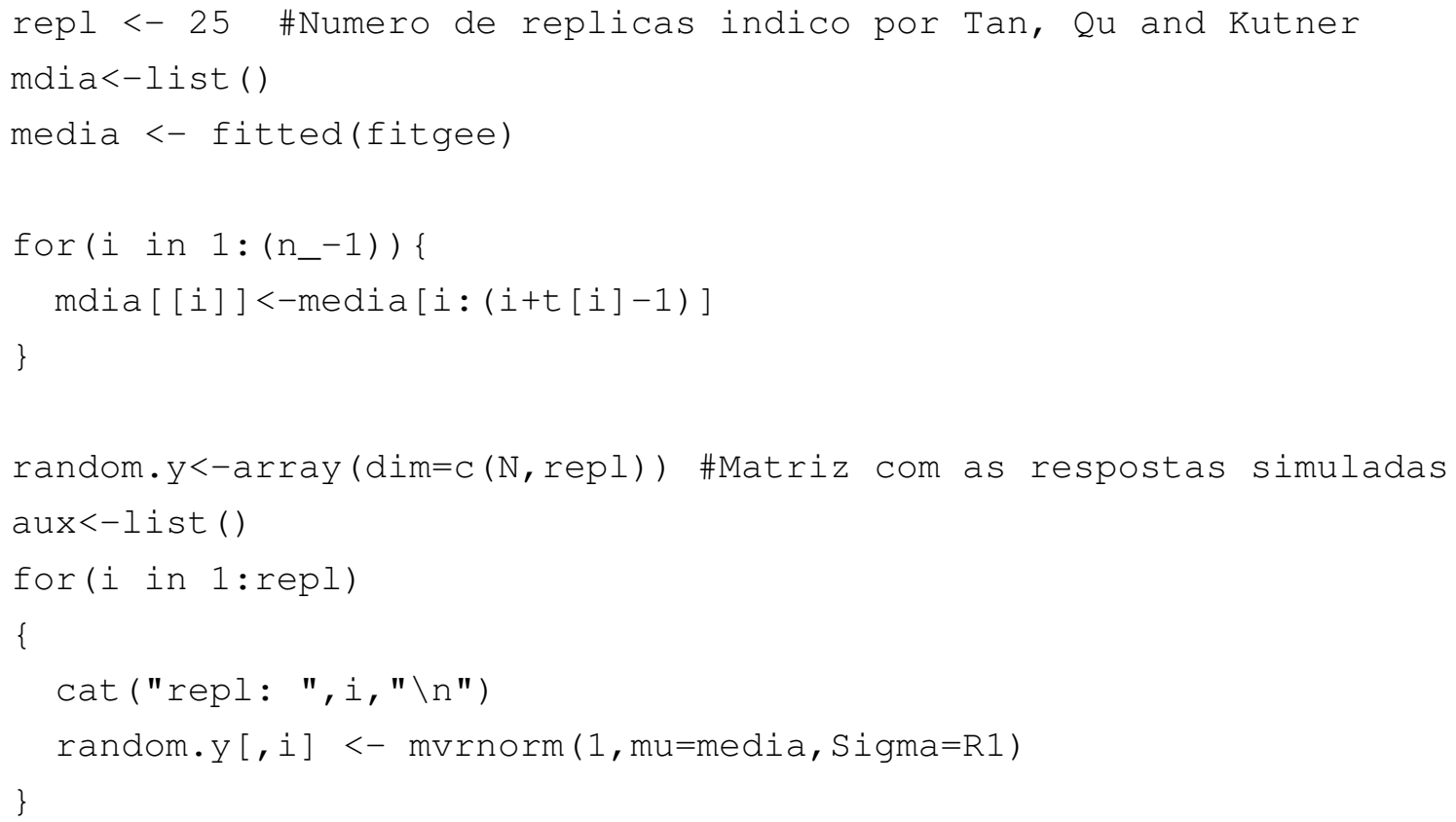




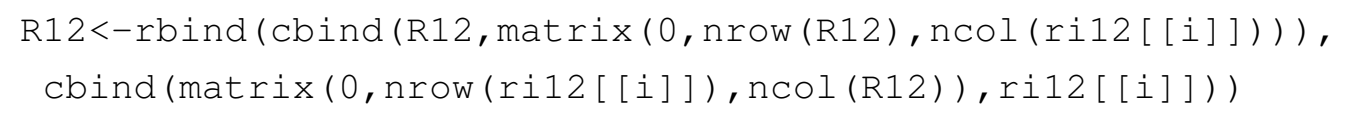




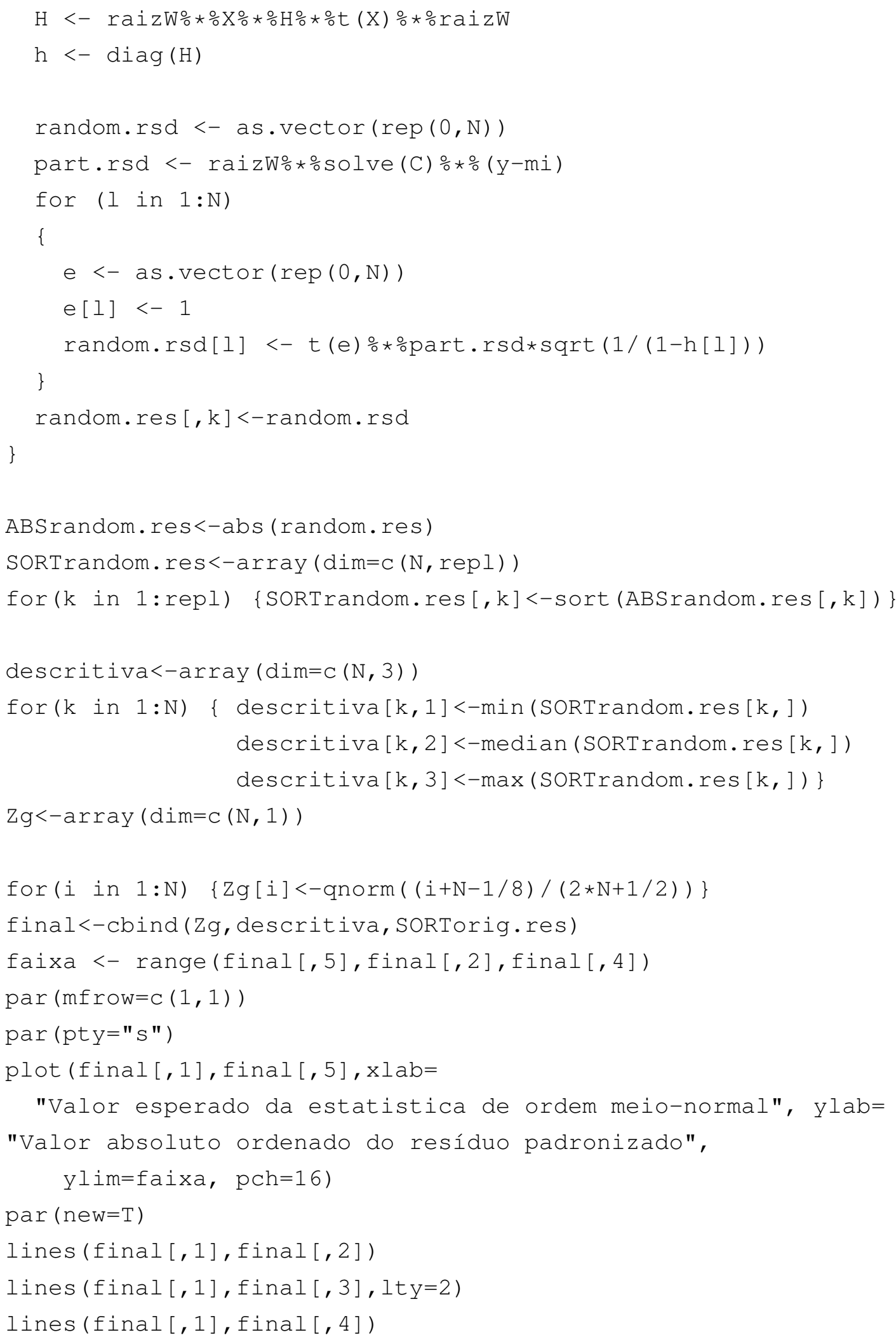




\section{MLGH gama:}

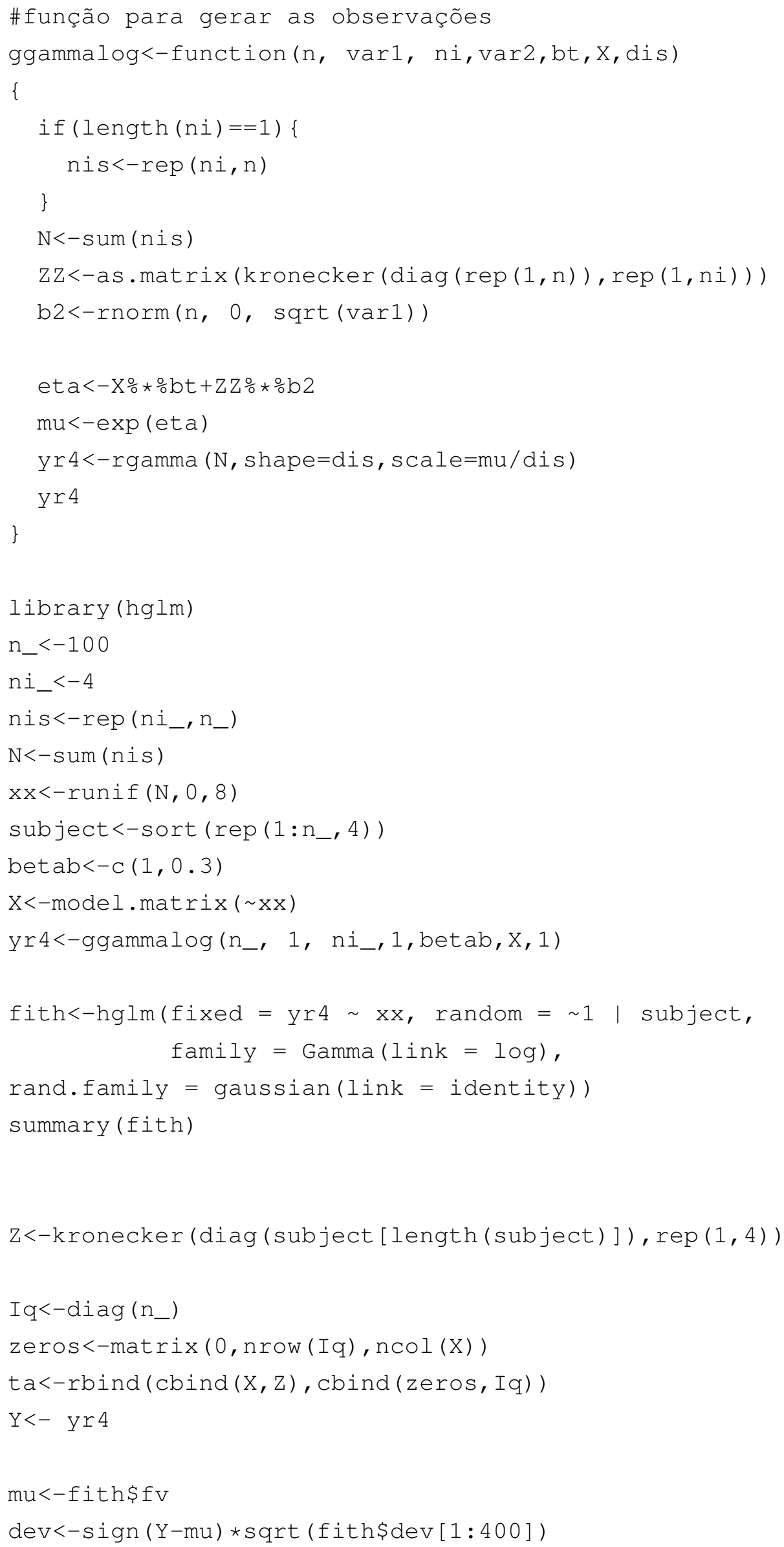




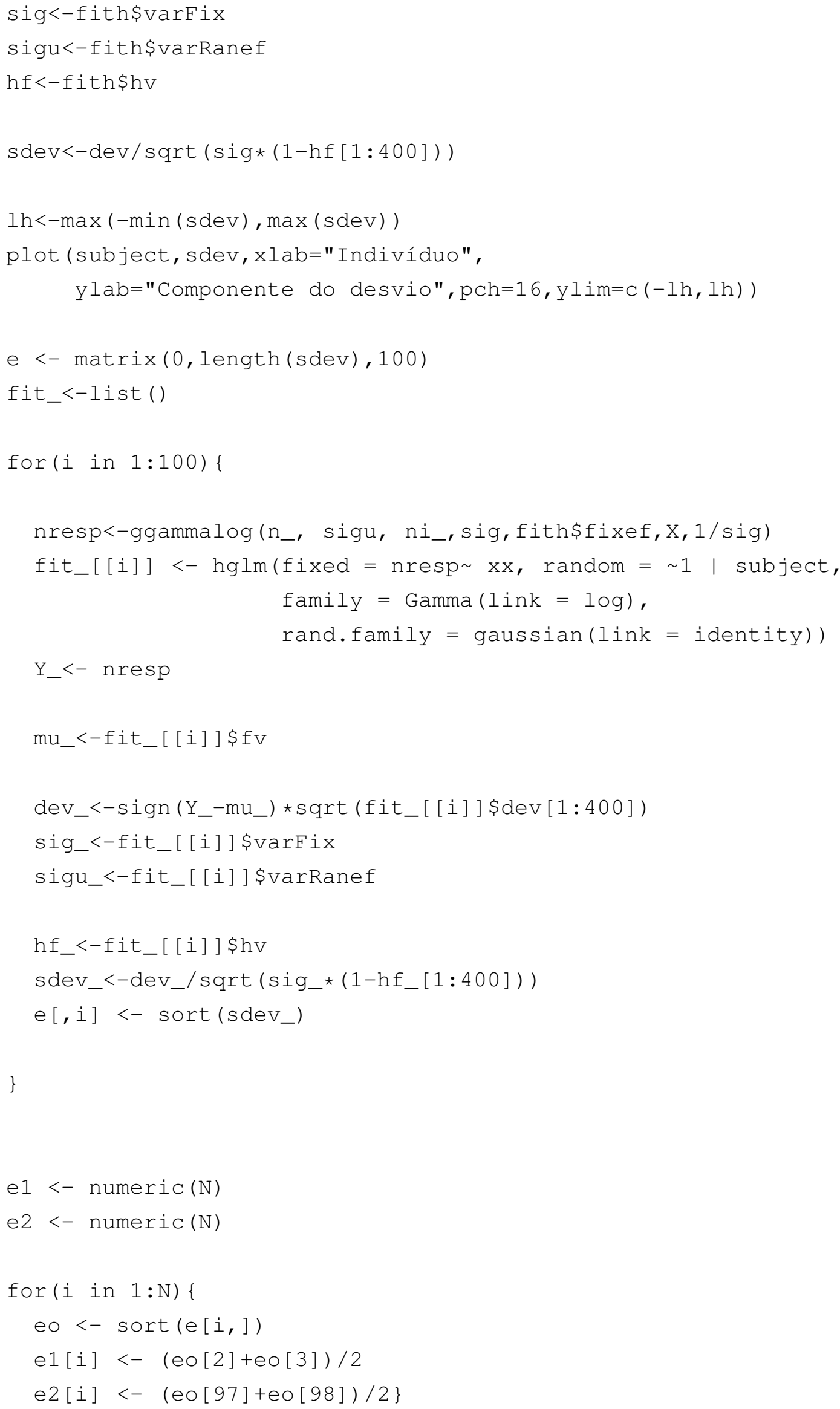


med $<-$ apply $(e, 1$, mean $)$

faixa <- range (sdev, e1, e2)

par $($ pty $=" \mathrm{~s} ")$

qqnorm(sdev, xlab="Percentil da $\mathrm{N}(0,1)$ ", ylab="Componente do desvio",ylim=faixa, pch=16, main=NULL) $\operatorname{par}($ new $=\mathrm{T})$

qqnorm (el, axes $=F, x l a b="$ ", y lab=" ", type="l ", ylim=faixa, lty=1, main=NULL)

$\operatorname{par}($ new $=\mathrm{T})$

qqnorm $(e 2$, axes $=F, x l a b="$ ",ylab=" , type="l", ylim=faixa, lty=1, main=NULL)

$\operatorname{par}($ new $=\mathrm{T})$

qqnorm(med, axes=F, xlab="", ylab="", type="l", ylim=faixa, 1 ty=2, main=NULL) 


\section{Referências Bibliográficas}

Artes e Botter(2005) R. Artes e D. A. Botter. Funções de estimação em modelos de regressão. ABE. Citado na pág. 27

Atkinson(1985) A. C. Atkinson. Plots, transformations, and regression: an introduction to graphical methods of diagnostic regression analysis. Clarendon Press Oxford. Citado na pág. 17

Bates(2012) D. Bates. Linear mixed model implementation in lme4. Ms., University of Wisconsin. Citado na pág. 85

Beckman et al.(1987) R. J. Beckman, C. J. Nachtsheim, e R. D. Cook. Diagnostics for mixed-model analysis of variance. Technometrics, 29(4):413-426. Citado na pág. 20

Bjørnstad(1996) J. F. Bjørnstad. On the generalization of the likelihood function and the likelihood principle. Journal of the American Statistical Association, 91(434):791-806. Citado na pág. 11

Breslow e Clayton(1993) N. E. Breslow e D. G. Clayton. Approximate inference in generalized linear mixed models. Journal of the American Statistical Association, páginas 9-25. Citado na pág. 1,10

Butler e Louis(1992) S. M. Butler e T. A. Louis. Random effects models with non-parametric priors. Statistics in medicine, 11(14-15):1981-2000. Citado na pág. 105

Demidenko(2004) E. Demidenko. Mixed models: theory and applications, volume 518. LibreDigital. Citado na pág. 9

Demidenko e Stukel(2005) E. Demidenko e T. A. Stukel. Influence analysis for linear mixedeffects models. Statistics in Medicine, 24(6):893-909. Citado na pág. 18

Diggle et al.(2002) P. Diggle, P. Heagerty, K. Y. Liang, e S. Zeger. Analysis of longitudinal data, volume 25. Oxford University Press, USA. Citado na pág. 2

Dunn e Smyth(1996) P. K. Dunn e G. K. Smyth. Randomized quantile residuals. Journal of Computational and Graphical Statistics, 5(3):236-244. Citado na pág. 17

Fung et al.(2002) W. K. Fung, Z. Y. Zhu, B. C. Wei, e X. He. Influence diagnostics and outlier tests for semiparametric mixed models. Journal of the Royal Statistical Society: Series B (Statistical Methodology), 64(3):565-579. Citado na pág. 19

Graybill(1983) F. A. Graybill. Matrices with applications in statistics. Belmont, CA: Wadsworth. Citado na pág. 14 
Gumedze et al.(2010) F. N. Gumedze, S. J. Welham, B. J. Gogel, e R. Thompson. A variance shift model for detection of outliers in the linear mixed model. Computational Statistics $\mathcal{G}$ Data Analysis, 54(9):2128-2144. Citado na pág. 19

Harville(2008) D. A. Harville. Matrix algebra from a statistician's perspective. Springer. Citado na pág. 6,16

Henderson(1973) C. R. Henderson. Sire evaluation and genetic trends. Em Proceedings of the animal breeding and genetics symposium in honor of Dr J. Lush, páginas 10-41. Citado na pág. 8

Hilden-Minton(1995) J. A. Hilden-Minton. Multilevel diagnostics for mixed and hierarchical linear models. Tese de Doutorado, University of California Los Angeles. Citado na pág. 15, 16

Hubbard et al.(2010) A. E. Hubbard, J. Ahern, N. L. Fleischer, M. Van der Laan, S. A. Lippman, N. Jewell, T. Bruckner, e W. A. Satariano. To gee or not to gee: comparing population average and mixed models for estimating the associations between neighborhood risk factors and health. Epidemiology, 21(4):467-474. Citado na pág. 2

Jiang(2007) J. Jiang. Linear and generalized linear mixed models and their applications. Springer Verlag. Citado na pág. 2

Johnson e Wichern(2007) R. A. Johnson e D. W. Wichern. Applied multivariate statistical analysis, volume 6. Prentice Hall. Citado na pág. 8

Laird e Ware(1982) N. M. Laird e J. H. Ware. Random-effects models for longitudinal data. Biometrics, páginas 963-974. Citado na pág. 1

Lee e Nelder(1996) Y. Lee e J. A. Nelder. Hierarchical generalized linear models. Journal of the Royal Statistical Society. Series B (Methodological), páginas 619-678. Citado na pág. 1, 10

Lee e Nelder(2001) Y. Lee e J. A. Nelder. Hierarchical generalised linear models: A synthesis of generalised linear models, random-effect models and structured dispersions. Biometrika, 88(4):987-1006. Citado na pág. 12

Lee e Nelder(2004) Y. Lee e J. A. Nelder. Conditional and marginal models: another view. Statistical Science, 19(2):219-238. Citado na pág. 2

Lee et al.(2006) Y. Lee, J. A. Nelder, e Y. Pawitan. Generalized linear models with random effects: unified analysis via H-likelihood, volume 106. Chapman \& Hall/CRC. Citado na pág. 13

Lesaffre e Verbeke(1998) E. Lesaffre e G. Verbeke. Local influence in linear mixed models. Biometrics, páginas 570-582. Citado na pág. 14, 20

Liang e Zeger(1986) K. Y. Liang e S. L. Zeger. Longitudinal data analysis using generalized linear models. Biometrika, 73(1):13-22. Citado na pág. 1, 23, 25

Manghi(2012) R. F. Manghi. Modelos elípticos multiníveis. São Paulo. Dissertação (Mestrado). Instituto de Matemática e Estatística. Universidade de São Paulo. Citado na pág. 85 
McCullagh e Nelder(1989) P. McCullagh e J. A. Nelder. Generalized linear models, volume 37. Chapman \& Hall/CRC. Citado na pág. 1

McCulloch e Neuhaus(2011) C. E. McCulloch e J. M. Neuhaus. Misspecifying the shape of a random effects distribution: why getting it wrong may not matter. Statistical Science, 26 (3):388-402. Citado na pág. 105

McCulloch et al.(2008) C. E. McCulloch, S. R. Searle, e J. M. Neuhaus. Generalized, Linear, and Mixed Models. Wiley, New Jersey, 2nd edição. Citado na pág. 2, 10

Molas e Lesaffre(2011) M. Molas e E. Lesaffre. Hierarchical generalized linear models: The r package hglmmm. Journal of Statistical Software, 39(i13). Citado na pág. 85

Nobre(2004) J. S. Nobre. Métodos de diagnóstico para modelos lineares mistos. São Paulo. Dissertação (Mestrado). Instituto de Matemática e Estatística. Universidade de São Paulo. Citado na pág. 18, 19, 85

Nobre e Singer(2007) J. S. Nobre e J. M. Singer. Residual analysis for linear mixed models. Biometrical Journal, 49(6):863-875. Citado na pág. 2, 13

Nobre e Singer(2011) J. S. Nobre e J. M. Singer. Leverage analysis for linear mixed models. Journal of Applied Statistics, 38(5):1063-1072. Citado na pág. 2, 18

Pardo e Alonso(2012) M. C. Pardo e R. Alonso. Influence measures based on the volume of confidence ellipsoids for gee. Biometrical Journal, 54(4):552-567. Citado na pág. 28

Park e Shin(1998) C. G. Park e D. W. Shin. An algorithm for generating correlated random variables in a class of infinitely divisible distributions. Journal of Statistical Computation and Simulation, 61(1-2):127-139. Citado na pág. 28

Patterson e Thompson(1971) H. D. Patterson e R. Thompson. Recovery of inter-block information when block sizes are unequal. Biometrika, 58(3):545-554. Citado na pág. 9

Paula(2012) G. A. Paula. Modelos de regressão: com apoio computacional. IME-USP Sao Paulo:. Citado na pág. 2

Paula et al.(2005) G. A. Paula, F. H. F. P. Rosa, e V. A. Pedro Júnior. Relatório de análise estatística sobre o projeto: Influência da vitamina a e da sazonalidade no sêmen suíno. Relatório técnico, RAE - CEA - 05P16. Citado na pág. 87

Pinheiro e Bates(2000) J. C. Pinheiro e D. M. Bates. Mixed-effects models in S and S-PLUS. Springer Verlag. Citado na pág. 17

Preisser e Qaqish(1996) J. S. Preisser e B. F. Qaqish. Deletion diagnostics for generalised estimating equations. Biometrika, 83(3):551-562. Citado na pág. 28

Raiffa e Schlaifer(1961) H. Raiffa e R. Schlaifer. Applied statistical decision theory. Division of Research, Harvard Business School, Boston, MA. Citado na pág. 11 
Raudenbush et al.(2000) S. W. Raudenbush, M. L. Yang, e M. Yosef. Maximum likelihood for generalized linear models with nested random effects via high-order, multivariate laplace approximation. Journal of computational and Graphical Statistics, 9(1):141-157. Citado na pág. 10

Rönnegård et al.(2010) L. Rönnegård, X. Shen, e M. Alam. hglm: A package for fitting hierarchical generalized linear models. $R$ Journal (accepted). Citado na pág. 85

Schützenmeister e Piepho(2011) A. Schützenmeister e H. P. Piepho. Residual analysis of linear mixed models using a simulation approach. Computational Statistics $\&$ Data Analysis. Citado na pág. 17, 21, 48

Tan et al.(2001) F. E. S. Tan, M. J. N. Ouwens, e M. P. F. Berger. Detection of influential observations in longitudinal mixed effects regression models. Journal of the Royal Statistical Society: Series D (The Statistician), 50(3):271-284. Citado na pág. 19

Twisk(2004) J. W. R. Twisk. Longitudinal data analysis. a comparison between generalized estimating equations and random coefficient analysis. European journal of epidemiology, 19 (8):769-776. Citado na pág. 2

Venezuela(2003) M. K. Venezuela. Modelos lineares generalizados para análise de dados com medidas repetidas. São Paulo. Dissertação (Mestrado). Instituto de Matemática e Estatística. Universidade de São Paulo. Citado na pág. 28, 85

Venezuela et al.(2007) M. K. Venezuela, D. A. Botter, e M. C. Sandoval. Diagnostic techniques in generalized estimating equations. Journal of Statistical Computation and Simulation, 77 (10):879-888. Citado na pág. 2, 27

Venezuela et al.(2011) M. K. Venezuela, M. C. Sandoval, e D. A. Botter. Local influence in estimating equations. Computational Statistics \& Data Analysis, 55(4):1867-1883. Citado na pág. 28

Verbeke e Lesaffre(1996) G. Verbeke e E. Lesaffre. A linear mixed-effects model with heterogeneity in the random-effects population. Journal of the American Statistical Association, 91(433):217-221. Citado na pág. 105

Verbeke e Lesaffre(1997) G. Verbeke e E. Lesaffre. The effect of misspecifying the randomeffects distribution in linear mixed models for longitudinal data. Computational Statistics $\&$ Data Analysis, 23(4):541-556. Citado na pág. 105

Waternaux et al.(1989) C. Waternaux, N. M. Laird, e J. H. Ware. Methods for analysis of longitudinal data: blood-lead concentrations and cognitive development. Journal of the American Statistical Association, 84(405):33-41. Citado na pág. 18

Xiang et al.(2002) L. Xiang, S. K. Tse, e A. H. Lee. Influence diagnostics for generalized linear mixed models: applications to clustered data. Computational statistics $\&$ data analysis, 40 (4):759-774. Citado na pág. 21 